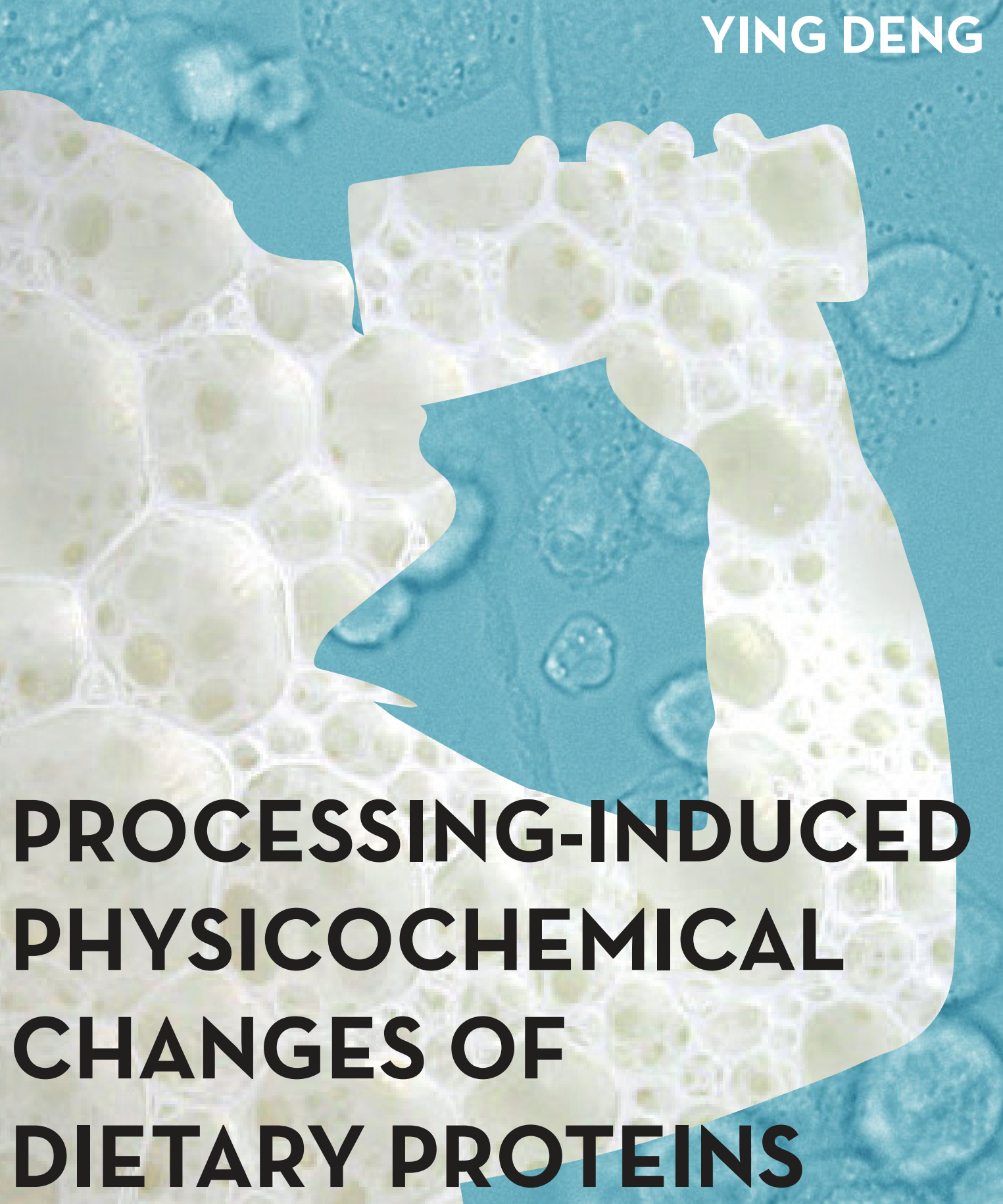

Implications for digestion,

intestinal uptake

and immunological responses 


\section{Propositions}

1. The observation that CMA-children tolerate "baked" milk better than raw milk is in line with our findings.

(this thesis)

2. Computational biology bears similarities to digestion: what is fed in will come out somehow, be it in a format that you like or not.

(this thesis)

3. Low-dose oral intake or extensive heating might be better options to prevent food allergy than strict avoidance of allergens (Yanagida et al. Allergol Int. 2016:65 135-140).

4. For concentration on a highly demanding task like writing a PhD thesis, the beneficial effect of classical music is not convincing (Janina et al. Front Psychol. 2017:8), but undoubtedly better if compared to a noisy background (e.g. frequent construction work in the building).

5. The benefit of napping to improve memory and performance has been supported scientifically (McDevitt et al., Sci Rep. 2018:8) and experienced personally (Ying Deng, Resource 2018:12 back cover).

6. After being proud of the art of cooking as a symbol of civilization for thousands of years, we probably finally figure out it is safer to eat food as raw as possible.

Propositions belonging to the thesis entitled:

Processing-induced physicochemical changes of dietary proteins: implications for digestion, intestinal uptake and immunological responses

Ying Deng

Wageningen, 30 June 2020 


\title{
Processing-induced physicochemical changes of dietary proteins: implications \\ for digestion, intestinal uptake and immunological responses
}

\author{
Ying Deng
}




\section{Thesis committee}

\section{Promotor}

Prof. Dr H.J. Wichers

Special Professor, Immune Modulation by Food

Wageningen University \& Research

\section{Co-promotors}

Dr K.A. Hettinga

Associate Professor, Food Quality and Design

Wageningen University \& Research

Dr C.C.F.M. Govers

Scientist, Fresh Food \& Chains

Wageningen University \& Research

\section{Other members}

Prof. Dr R.J.J. van Neerven, Wageningen University \& Research

Prof. Dr J. Garssen, Utrecht University

Dr K. Broersen, University of Twente, Enschede

Dr C. Soler Rivas, Universidad Autonoma de Madrid, Spain

This research was conducted under the auspices of the Graduate School VLAG (Advanced studies in Food Technology, Agrobiotechnology, Nutrition and Health Sciences) 


\title{
Processing-induced physicochemical changes of dietary proteins: implications for digestion, intestinal uptake and immunological responses
}

\author{
Ying Deng
}

Thesis

submitted in fulfilment of the requirements for the degree of doctor

at Wageningen University

by the authority of the Rector Magnificus,

Prof. Dr A. P. J. Mol,

in the presence of the

Thesis Committee appointed by the Academic Board

to be defended in public

on Tuesday 30 June 2020

at 11 a.m. in the Aula. 
Ying Deng

Processing-induced physicochemical changes of dietary proteins: implications for digestion, intestinal uptake and immunological responses,

157 pages.

PhD thesis, Wageningen University, Wageningen, the Netherlands (2020)

With references, with summary in English

ISBN: 978-94-6395-381-8

DOI: $10.18174 / 519803$ 


\section{Table of contents}

Chapter 1 General introduction

Chapter 2 Heat treatment of $\beta$-lactoglobulin affects its survival and translocation in the upper digestive tract

Chapter 3 Hydrophobicity and aggregation, but not glycation, are key determinants for uptake of thermally processed $\beta$-lactoglobulin by THP-1 macrophages

Chapter 4 A THP-1 cell line-based exploration of immune responses towards 75 heat-treated BLG

Chapter 5 Hydrophobicity drives receptor-mediated uptake of heat-processed proteins by THP-1 macrophages and dendritic cells, but not cytokine responses

Chapter 6 Discussion

Summary

Acknowledgements

About the author 

Chapter 1

General introduction 


\section{Food processing and its effects on protein}

Before consumption, food products are often processed in various ways like pickling, cooking, mincing and fermentation to improve their nutritive value, sensory attributes, and extend their shelf life. In order to avoid quality problems like undesired microorganisms in food products, reliable disinfection techniques are used, e.g. high-pressure processing, ultrasound processing, and most frequently thermal processing [1]. In addition to preservation, thermal processing helps to provide consumers satisfaction by enhancing food's palatability and flavour. There are various types of thermal processing, of which pasteurization and sterilization are the two main types [2].

Protein, as a macronutrient from our food, is among the most important biochemical compounds for living organisms. A daily intake of $0.8 \mathrm{~g}$ protein $/ \mathrm{kg}$ body weight is commonly advised [3]. Protein is not only the building block that delivers amino acids to form muscles, skin, blood and internal organs, but also an essential component for the body's regulation systems (function as enzymes and hormones) [4]. Digestion and absorption are essential steps for breaking down food proteins for further reuse of the constituting amino acids. Digestibility and bioavailability of protein can be altered by heating, mainly due to heat-induced denaturation and aggregation [5]. A higher digestibility of egg, linseed and meat proteins was found after heating [6-8]. Besides contributing to protein metabolism, food proteins also have a direct impact on human health, which can be modulated by heat processing of the protein. For example, the health promoting impact of bioactive peptides in a number of foods was reduced by different heat treatments [9]. Undesirable or even toxic amino acid derivatives like lysinoalanine and D-amino acids may also be formed after thermal processing, depending on the specific heat treatment used [10].

\section{Industrial milk processing}

Thermal processing of milk is an essential step in the dairy industry [11] in which the heating conditions vary depending on the specifications and demands for target products. Pasteurization, for instance, takes place at a relatively low heating temperature of around $70^{\circ} \mathrm{C}$ for $15-20$ seconds and is often used for liquid milk products. In-package and ultra-high temperature (UHT) sterilization operate at $110^{\circ} \mathrm{C}$ for 10-30 minutes and above $130^{\circ} \mathrm{C}$ for seconds, respectively [12]. Spray drying with an inlet temperate of approximately $130^{\circ} \mathrm{C}$, and an outlet temperature of approximately $60^{\circ} \mathrm{C}$, is currently the most common process to obtain dry products like infant formulas [13]. Strong intermolecular interactions between proteins, lipids and/or saccharides are often observed in heat-treated cow's milk [14], including aggregation and glycation: the two main reactions interfering with the structural characteristics of the protein. 


\section{Aggregation}

During its ribosomal synthesis, a protein is folded into its unique three-dimensional structure, which is a prerequisite for it to be functional. An energy landscape theory has been proposed describing a shift towards a low energy phase when protein is converted from the unfolded state into its native conformation (Figure 1.1) [15]. When sufficient energy inputs are applied, such as heating, high or low $\mathrm{pH}$ or oxidative agents, a protein can be unfolded (thermodynamically high state), which then has the tendency to achieve a lower energy level and so aggregates formed.

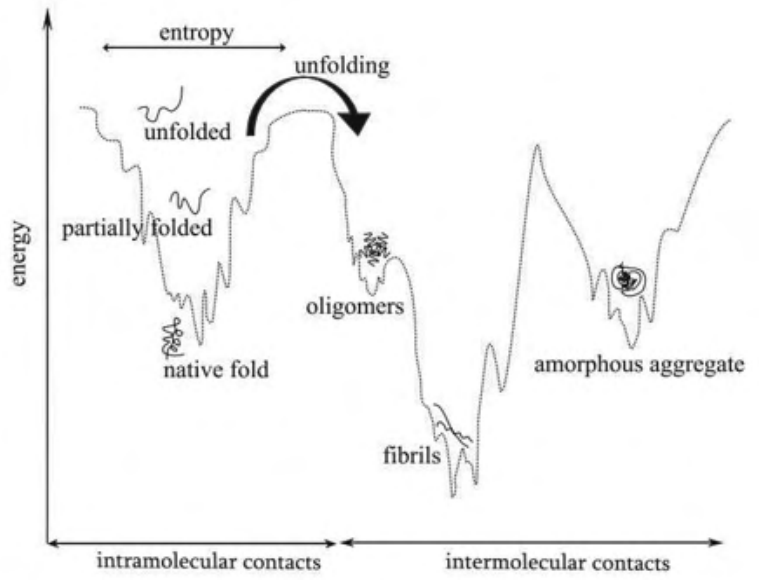

Figure 1.1 Energy state of protein under native and aggregate conditions. After triggering by a sufficient energy input to unfold, the native protein has the tendency to unfold and aggregateachieving a lower energy state. Figure from Herczenik et al. [15].

Heat induced aggregation and structural modifications of food proteins occurs commonly in daily life, for instance the 'solidification' of egg proteins after boiling or frying. It is also widely observed in processed milk products. For instance, heating of whey protein in solution above $70{ }^{\circ} \mathrm{C}$ for a few minutes led to a higher viscosity and particle size than the untreated control, due to protein denaturation and aggregation $[16,17]$.

\section{Maillard Reaction}

The Maillard reaction is a complex non-enzymatic reaction between a reducing sugar (e.g. glucose) and the free amino group of a protein. It can be divided into three stages: the initial, the advanced and the final stage. In the initial stage, also known as glycation, the carbonyl group of a reducing sugar attaches to a free amino group of the protein, mainly from lysine and arginine residues, or the $\alpha$-amino group 
of the N-terminal amino acid. As shown in Figure 1.2, an unstable Schiff base is formed which irreversibly rearranges into an Amadori product. In the advanced stage, the Amadori product is degraded and subsequently converted into reactive $\alpha$-carbonyl compounds. Via further reactions with additional free amino groups, advanced glycation end products (AGEs) are formed from the $\alpha$-carbonyl compounds [18]. In the final stage of the Maillard reaction, brown coloured compounds with high molecular weight, such as melanoidins, are formed [19].

The extent of the Maillard reaction depends on the reactivity of the sugar. For example, short chain reducing sugars will be leading to more AGEs compared to long chain sugars, under identical conditions [20]. Thus, abundant Maillard reaction products (MRPs) can be formed in milk products, due to the presence of the short chain reducing sugar lactose. It was also shown that low water activity and the application of high temperature would enhance the formation of AGEs [21]. Such conditions are very similar to conditions during the production of dry milk products. AGEs like $N(\varepsilon)$-carboxymethyllysine (CML), pyrraline and pentosidine are commonly reported in milk products [22-24].
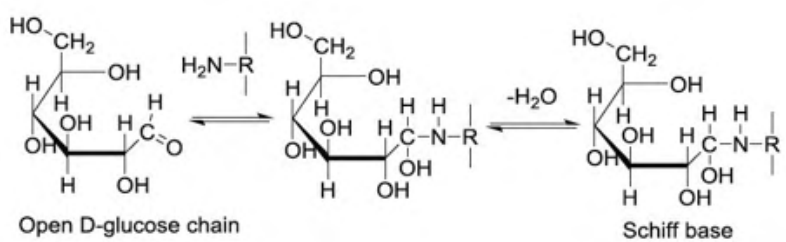

Open D-glucose chain

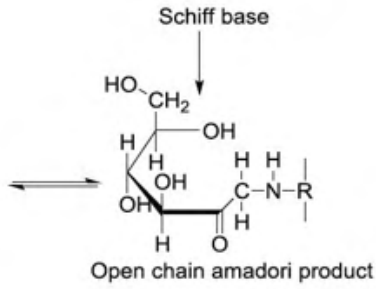

Figure 1.2 Schematic representation of the initial stage of the Maillard reaction, from Zhang et al. [25]. Nucleophilic attack by a free amino group on the aldehyde of a reducing sugar (e.g. glucose) leads to the formation of an unstable Schiff base which rearranges into an Amadori product.

\section{Food allergy}

Food allergy is an adverse immune response that occurs after consumption of certain food. It affects about $5 \%$ of the children in western countries and the prevalence keeps increasing [26, 27]. The development of food allergy starts when an immune cell encounters a food allergen (normally protein) in the gastrointestinal (GI) tract, oral cavity, skin or respiratory tract for the first time [28]. Antigen 
presenting cells (APCs), such as macrophages and dendritic cells (DCs), engulf the allergen in a vesicle called a phagosome [29], which processes it into linear peptides. These peptides will be presented to Thelper cells (Th cells) via MHC class II molecules on the cell surface, which might cause the activation of an adaptive Thelper type 2 (Th2) cell dominated response [30]. Once activated, Th2 cells will secrete cytokines like IL-4 and IL-13 which would lead to IgE antibody production by B cells [29]. The attachment of such allergen-specific IgE antibodies to FceRI receptors on the surface of mast cells and basophils is called allergic sensitization. The elicitation step starts when a second exposure with the same allergen occurs. This then is recognized and bound by the specific receptor bound antibodies on the surface of the mast cells and basophils. When crosslinking of specific IgE occurs, chemical mediators such as histamine will be released [31]. The release of such mediators will lead to a series of allergic responses, including asthma, rhinitis, urticaria and anaphylaxis [32].

\section{Cow's milk allergy}

As mentioned before, the Gl tract is one of the main sites for the development and allergic manifestations of cow's milk allergy (CMA), which is one of the most prevalent food allergies in the world. The prevalence of CMA is around $2-5 \%$ in early childhood $[33,34]$ and decreases to $0.1-0.5 \%$ in adulthood [35]. Cow's milk contains about $3 \%$ protein, which consists of hundreds of different proteins [36], of which at least 9 proteins (4 caseins and 5 whey proteins) are known to be allergenic [37]. Twenty percent of the total cow's milk protein is whey proteins and almost half of the whey proteins is $\beta$-lactoglobulin (BLG), one of the major allergens for CMA [38] together with $\alpha$-lactalbumin (ALA). Unlike other whey proteins or casein, BLG is absent in human milk [39]. BLG is a small globular protein and naturally occurs in the form of a 36-kDa dimer at neutral $\mathrm{pH}$ and ambient temperatures. Each of its subunits consists of 162 amino acids, with 2 disulphide bonds and 1 free cysteine [39, 40]. Caseins are also reported to be important allergens in $\mathrm{CMA}$, of which $\alpha_{\mathrm{S} 1}$-casein is suspected to be the most allergenic [41].

Besides the clearly identified IgE mediated mechanism, as described previously, CMA could also be generated through a non-IgE mediated pathway. Unlike the rapid response of IgE mediated CMA (symptoms occurring a few minutes to hours after allergen contact), the hypersensitivity of the nonIgE mediated CMA is delayed (hours to days) and becomes more dominant with increasing age [42]. The mechanism behind this, in which T lymphocyte subsets and eosinophils are suspected to play the main role, is still under discussion [43, 44].

There is little doubt that thermal processing influences the immunogenicity of cow's milk in CMA. Yet there is still uncertainty about whether thermal processing increases or reduces the allergenicity and 
antigenicity of cow's milk. Processing of milk was found to either reduce or enhance its allergic potential depending on the method of processing [45]. For example, heating milk at $80{ }^{\circ} \mathrm{C}$ for 10 minutes was shown to induce exacerbated allergic symptoms, such as dermal responses, anaphylactic shock, IgE level and Th2 cytokines in whey protein sensitized mice, and lower the threshold levels in CMA children [46]. In another study, CMA children were shown to be more tolerant to extensive heated milk $\left(>170^{\circ} \mathrm{C}\right)$ than to raw milk [47].

\section{Influence of denaturation and aggregation on milk protein allergenicity}

Allergens contain conformational and/or linear epitopes (unique amino acids sequence), which are essential for immunological recognition. Aggregation has a strong influence on the allergenicity of milk protein through the modification of CMA epitopes, where especially conformational epitopes can be abolished due to aggregation. Lower allergenicity of aggregated peanut allergen and other food proteins was observed [48, 49]. After heating, BLG and ALA which both have conformational epitopes were reported to have reduced antigenicity $[50,51]$. On the other hand, the antigenicity of the mainly linear epitopes containing allergen casein was hardly influenced by the heating [52]. Besides the destruction of epitopes that happens in aggregation, epitopes that are normally buried inside the native structure of a protein may also be exposed due to the denaturation. There is also quite some evidence that aggregation could lead to the formation of new epitopes [53]. BLG is reported to have multiple new epitopes after heat-induced denaturation [54]. This might explain the observation of increased antigenicity of BLG and ALA with increased heating up to $90{ }^{\circ} \mathrm{C}$ [51]. Higher antigenicity of BLG was also found when the protein was unfolded using dynamic high-pressure microfluidization [55].

\section{Influence of Maillard reaction on milk protein's allergenicity}

Similar to denaturation and aggregation, the allergenicity of food proteins after being subjected to the Maillard reaction could also be influenced by the resulting disturbance of the conformational structure and the formation of complex mixtures of reaction products with reducing sugar from the food matrix [56]. In addition to modifying conformational epitopes due to structure changes, linear epitopes may also be disturbed due to the binding of a reducing sugar. The allergenicity of milk protein was widely reported to decrease after glycation [57]. Conjugation of the lysine from BLG with a reducing sugar (e.g. lactose) weakened its binding to IgE from CMA patients due to the masking of epitopes [58]. Two other studies confirmed the masking of epitopes being related to the reduced allergenicity of glycated $\operatorname{BLG}[59,60]$. The extent of reduced allergenicity of BLG was related to the extent of the glycation reaction and the size of the reducing sugar, which further confirms the essential role of epitope masking $[61,62]$. On the other hand, new epitopes can also be formed or exposed during Maillard 
reaction due to structure changes, similar to aggregation. For instance, $N(\varepsilon)$-carboxylmethyllysine $(\mathrm{CML})$, one of the main AGE structures, was shown to be a novel allergenic epitope [63].

\section{GI tract and its immunity}

The GI tract is not only an essential part of the digestive system, but also one of the main surfaces where food allergens interact with immune cells. Thus, it is important to understand the influence of digestion of food proteins to better estimate a protein's immunogenicity. Usually, a large proportion of food proteins would be digested by gastric and intestinal enzymes. However, a fraction of remaining sufficiently large peptides and proteins could pass through the intestinal epithelial cells or be sampled directly by APCs like DCs, which may induce a reaction of the gut-associated lymphoid tissue (GALT). $M$ cells, a specific type of epithelial cells, play an essential role in sampling structures in the lumen and transporting them to the mucosa to be examined by the immune system. M cells are located above Peyer's patches, a structure consisting of lymphoid follicles and occupied by various immune cells, including B cells, T cells, macrophages and DCs [64]. The immunogenicity of a protein strongly depends on their accessibility to the immune system in the GI tract. A correlation between epithelial barrier permeability and food allergy is found in both mouse and human studies [65]. The protein transport path across the small intestine also differs in food allergy sensitised and non-sensitised persons [66].

\section{The influence of processing on protein digestibility}

Digestion is a rather complex process performed mainly in the Gl tract. Digestive enzymes like pepsin, chymotrypsin, trypsin and elastase, are involved in hydrolysing dietary protein into peptides and amino acids [67]. Due to ethic and economic reasons in vitro models are used widely instead of animal or human trials. In protein digestion studies, a two-step system consisting of a gastric and an intestinal phase is most commonly used [68], while the oral phase is often excluded because the influence of salivary amylase on protein digestion is limited. Although from a qualitative perspective, the enzymes involved in digestion are quite clear, quantitative aspects such as enzyme activity, initial protein concentrations, type of digestion fluids, reaction time and pH vary among studies [69-74], introducing biases and variation in outcomes. Therefore, the consensus in vitro digestion protocol INFOGEST (http://www.cost-INFOGEST.eu/) was developed recently which involved more than 35 countries, and is now widely used for multi-domain research and has been cited over 650 times in Web of Science [75]. As expected, reduction of result variation was observed by the comparable results upon skim milk digestion in several laboratories using the INFOGEST protocol [76]. In addition, the findings from the INFOGEST protocol were corroborated by in vivo digestion results [77]. Milk proteins are highly digestible, with caseins and ALA mainly being digested in the gastric phase and BLG in the intestinal 
phase. BLG has a well-characterized tertiary structure, consisting of an 8-stranded antiparallel $\beta$-barrel with a 3-turn $\alpha$-helix on the outer surface and a ninth $\beta$-strand flanking the 1 st strand [78]. Due to its compact structure, BLG is relatively resistant to acid hydrolysis and proteolytic enzyme cleavage, resulting in resistance to gastric digestion. Thus, BLG would reach and be degraded to a large extent in the intestinal tract, which is contributing to its high allergenic potential [39, 78].

As mentioned above, processing would lead to various structure modifications of food proteins which will have an influence on protein's digestion as well. Mostly, protein's digestibility would increase after heat-induced unfolding and aggregation and decrease at further elevated temperature (normally above $100^{\circ} \mathrm{C}$ ), probably due to formation of insoluble particles [79] or the progression of the Maillard reaction [81]. As studying the immunogenicity of protein is the objective of this thesis, the focus is particularly on gastric digestibility rather than total digestibility. As gastric digestibility determines the amount and degree of digestion of protein that enters the intestinal tract where its immunogenicity would be manifested. For example, heating processes would not change the total digestibility (gastric + intestinal) of milk proteins but have an influence on gastric digestion [80]. Aggregated whey protein was observed to be digested faster in the gastric phase than native protein, and BLG becomes more gastric digestible after denaturation [72] [80]. On the contrary, a decrease of digestibility after glycation of proteins was observed as the low reactivity of digestive enzymes towards the potential cleavage sites which have been modified by glycation. For example, it was observed that the hydrolysis of ALA by gastric digestive enzyme decreased with increased degree of glycation [81].

\section{The role of intestinal antigen presenting cells}

Upon continuous encounters with foreign molecules, including those from food, it is essential for the host's immune system to balance its response between potential pathogens and harmless antigens. $\mathrm{A}$ disturbance of this delicate equilibrium may be the basis of various inflammatory diseases, including food allergy and inflammatory bowel disease. Intestinal macrophages and DCs play a dominant role in this antigen identification procedure of 'friend or foe' $[82,83]$. Both cell types being derived from bone marrow progenitors, macrophages and DCs differ from each other after being stimulated differently in the tissue [84]. Although both are professional phagocytes with a similar general structure and morphology, there are essential functional differences between macrophages and DCs, as detailed in Figure 1.3. It is possible to distinguish human DCs from macrophages via surface markers. The cellular surface of DCs is equipped with CD209, CD1c, CD11b and lacking CD14, CD64, CD163, contrasting to macrophages $[85,86]$. Furthermore, they play slightly different roles in the immune system. Macrophages are mainly responsible for innate immunity, scavenging pathogens, controlling the inflammatory response and recruiting $T$ cells and $B$ cells which will conduct adaptive immune 
responses. Although also being phagocytic, DCs are more specialized for antigen presentation and delivering signals to adaptive immune cells than macrophages due to the unique properties of the phagosome they formed after engulfing antigens. The phagosomes formed by DCs have neutral pH, low proteolytic and low oxidase activity, enabling them to preserve the antigens well [87]. Lastly, the tissue location of these two types of cells also differs. The common precursors of macrophages and DCs from bone marrow, monocytes, arrive at the gut via the blood circulation. After being differentiated in the lamina propria, macrophages normally settle locally and maintain the homeostatic microenvironment via phagocytosing potential pathogens and secreting regulatory cytokines [88]. DCs, on the other hand, have the unique ability to migrate to lymph nodes (chemokine receptor CCR7 dependent) and trigger the naïve T cells [89].

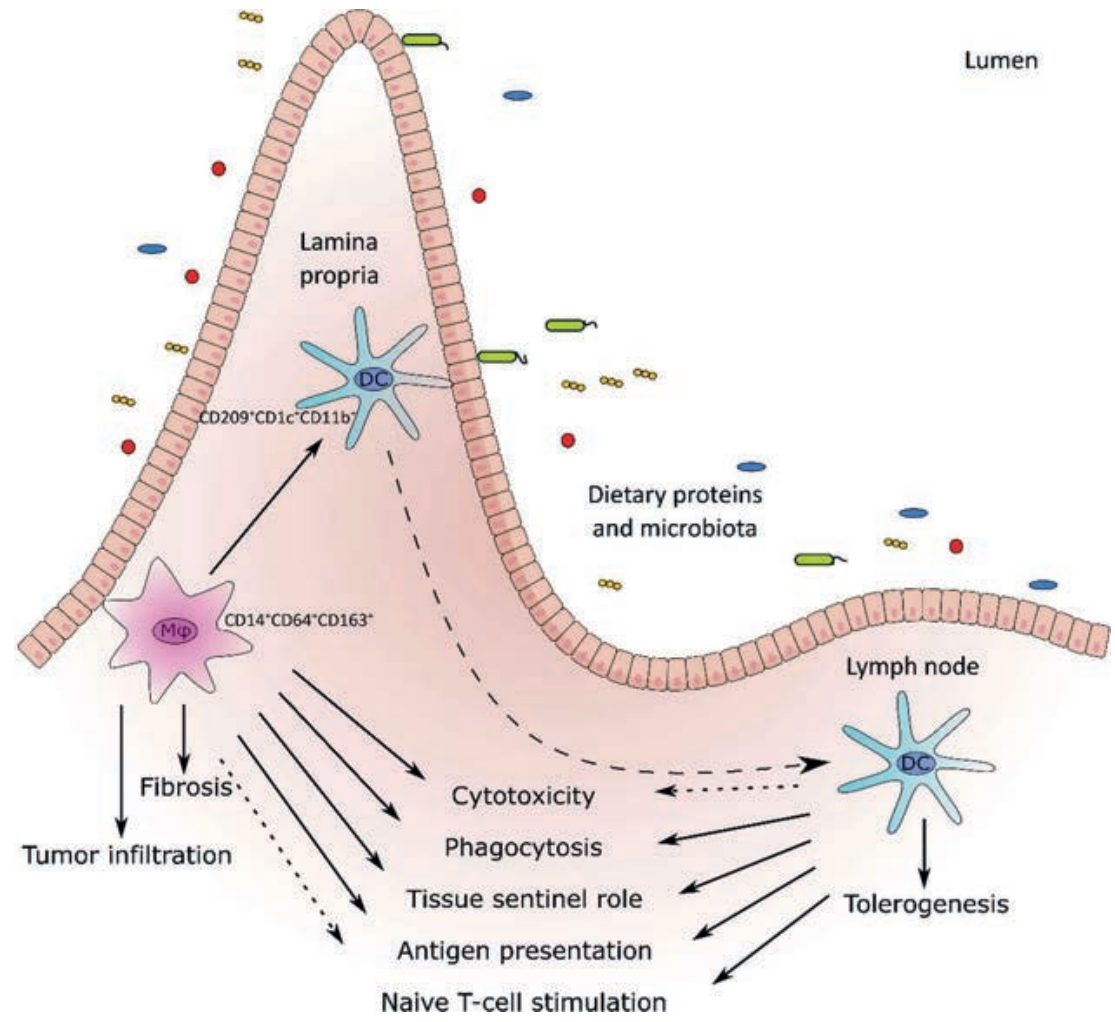

Figure 1.3 Similarities and differences in characteristics of intestinal macrophages $(M \phi)$ and dendritic cells (DCs). Macrophage and DC both inhabit in the lamina propria and sample the antigens from food in the intestine. Macrophage focuses more on phagocytosis and scavenging, which can lead to inflammation. After receiving a signal from macrophage or stimulated by a food antigen, DC could migrate to lymph node, delivering messages to $T$ and $B$ cells and contributing to immune tolerance or activation. Figure modified from Ferenbach et al. [94]. 
Macrophages and DCs both play essential roles in modulating immune responses when the body is exposed to potential food allergens. Macrophages can secrete not only IL-10 to establish tolerance, but also IL-1 $\beta$ to enrol innate lymphoid cells and DCs to activate regulatory $B$ cells and regulatory $T$ cells, which are essential for auto-immune suppression [90]. Moreover, DCs could also secrete IL-12 to induce T-helper type 1 (Th1) cells to inhibit sensitization to food allergens. Besides contribute to the development of food tolerance, macrophages and DCs are pivotal for allergic reaction. In an allergic situation (Th2 response), DCs sample and process the allergen, migrate to lymph nodes and activate T cells via interaction between CD86 from DCs and CD28 on T cells [90]. TNF $\alpha$, IL-6, GM-CSF, and IL-10 were found to be produced in high levels by activated DCs in the situation of CMA [91]. Macrophages, which are more efficient in antigen capturing and presenting, could deliver the antigen to DCs and active T cells [92]. Macrophages might also directly interact with T-helper cells, having the potential to induce a Th2 response [93].

\section{Immunomodulatory effects of processed cow's milk}

During the immune sensitisation step, food proteins are firstly phagocytosed by intestinal APCs after ingestion. After migration to lymph nodes, APCs could activate Th cells (Th0) either into the Th1 phenotype by IL-12 or the Th2 phenotype by IL-4. Th1 secreted cytokines (IL-2, IL-18 and IFNY) are proinflammatory and tend to induce an immune response which would eliminate the potential pathogens, while Th2 cytokines (IL-4, IL-5, IL10 and IL-13) are anti-inflammatory and promote a humoral immune response, i.e. antibody production [95]. The polarisation of Th0 cells into either Th1 or Th2 is irreversible and the cross-regulation between those two phenotypes could be further regulated by Treg, Th17 and Th22 [96]. The balance between Th1 and Th2 is critical for establishing allergen tolerance, allergy being more prone to develop when a Th2 > Th1 imbalance occurs [97].

Consumption of raw cow's milk is commonly associated with a decreased incidence of allergy in children's later life [98]. This might be due to the enhanced production of Treg cells after consumption of raw milk [99]. Cow's milk is abundant in immune related bioactive components that are active across species and are able to suppress or enhance human immune responses. Such components include vitamins, milk fat globule membranes, oligosaccharides, and many different proteins. A statistically significant inverse association was found between asthma and whey protein but not for lactoferrin, IgG or TGF- $\beta$, suggesting the protective effect of specific, but yet unknown, whey proteins in the development of allergy [100]. 


\section{Influence of aggregation on milk protein immunogenicity}

Aggregated milk proteins were more taken up via Peyer's patches than native proteins, thus facilitating direct interactions with immune cells [101]. A similar observation was reported for aggregated BLG with lower epithelial uptake but higher sampling through Peyer's patches compared to native BLG [102]. Besides being more accessible to intestinal immune cells, protein aggregates could also induce a different immune response compared to their native form. Aggregates of milk proteins as a result of pasteurization seemed to enhance the production of Th2-associated antibodies and cytokines in mice [101].

Although there are ample observations about the influence of aggregation on proteins' immunomodulatory effect, the mechanism and key determinants are not completely clear. Aggregation-induced size increase may alter the immune response. Immunogenicity of aggregated pharmaceutical proteins and inhalant allergens were increased, possibly due to the presence of microparticles $[103,104]$. However, when the particle size exceeded a certain limit $(5 \mu \mathrm{m})$, a reduction of size-enhanced Th2 response occurred [104]. Innate immune cells such as DCs are found to be less modulated by large particles than by smaller ones, probably as a consequence of the lower efficiency in uptake and lower activation of the MHC class II structure on the cell surface for the large particles[105-108]. Both inflammatory and anti-inflammatory molecules were secreted by macrophages treated with aggregated protein, and the production of IL-12, IL-10 and IL-23 by macrophages triggered by medium-size aggregates was even higher than in response to large aggregates [109]. Hydrophobicity alteration might also be an explanation for the effect of aggregation on the immune response. Hydrophobic regions, which are normally buried inside the native structure of protein, will be exposed during aggregation. Damage-associated molecular patterns (DAMPs), also known as alarmins, are molecules which could initiate the inflammatory response of the innate immune system. According to Matzinger et al. [110], hydrophobicity is an ancient DAMP which could induce an innate immune response.

\section{Influence of Maillard reaction on milk protein immunogenicity}

There are fewer studies on the immunomodulatory effect of proteins after glycation. This may be due to the limited structural modification in the early stage of Maillard reaction. But a specific product of advanced glycation (CML) was widely associated with diabetes and other immune disorder-related diseases $[111,112]$. AGEs are suspected to have the ability to activate DCs, resulting in a "false alarm" and promoting sensitization and development of food allergy [113]. The uptake of advanced glycated ovalbumin by DCs was found to be higher than that of native ovalbumin, leading to an increased 
production of IL-6 and transcription factor NF-KB which would further enhance the T cell immunogenicity and Th2 differentiation ability. This process was reported to be probably mannose receptor- and the scavenger receptor mediated [114-116]. Although blockage of the receptor of advanced glycation end products (RAGE) did not have an effect on the uptake, RAGE was found to be involved in the induction of a Th2-skewing milieu by DCs treated with AGEs in other studies [117, 118]. Moreover, the C-type lectin receptor DC-SIGN was involved in DC and Th2 stimulation upon treatment with Ara h 1, a natural glycoprotein allergen in peanut [119].

\section{Thesis outline}

The main focus of this thesis was to study the effects of structural modifications of food proteins on their immunogenicity, with a focus on the innate immune system. The significance of these structural modifications as a result of processing, and its consequences in triggering the innate immune system, is relatively underexposed. We hope to contribute from a food technological perspective to the development of tools to estimate and minimise the immunogenicity of processed food proteins, to hopefully contribute to reducing the incidence and prevalence of CMA. The major allergen of cow's milk, $\beta$-lactoglobulin (BLG), was treated by three heating methods that induce a range of structural modifications, to study the connection between these structural modifications and the innate immune response to the modified proteins.

The intestine is the main surface where exogenous proteins would interact with immune system, and where an impact of the digestive process on their immunogenicity is likely to happen. Chapter 2 therefore studies the digestibility of native and processed BLG based on an in vitro digestion model and several important physicochemical properties were measured along the whole digestion. Moreover, the intestinal epithelial monolayer was mimicked by a Caco-2 insert transwell system with FITC labelled samples added to the apical side. The amount and exact size of proteins that passed through the monolayer were measured. Combined with the previous digestion experiment, we can obtain a basic understanding about the size and quantity of protein that will react with intestinal immune cells. Chapter 3 describes physicochemical properties of native and heat-processed BLG. Also, the uptake of FITC-labelled BLG by THP-1 derived macrophage was measured. A correlation analysis was conducted to find the intricate relations between the physicochemical characteristics of the BLG and its uptake. In Chapter 4, the gene transcription and expression profile of macrophages and DCs derived from THP-1 were measured to characterise these cell types and to compare them with the properties of primary macrophages and DCs. Moreover, a microarray-based gene transcription profile was determined for these two types of cells and also their response after incubation with native and processed BLG samples. Gene transcription levels of some cytokines were verified by ELISA. To obtain 
a more comprehensive understanding of the parameters that are important determinants of uptake, in Chapter 5 we applied the same heating method and uptake measurements to other proteins, lysozyme and thyroglobulin due to their similarity, respectively differences, in molecular weight and pl with BLG. Correlation analysis was also applied to data of these two proteins to determine whether the underlying mechanisms of BLG for THP-1 macrophage uptake is applicable more universally. Taken the uptake capability of all 3 proteins by THP-1 derived macrophage and dendritic like cells together, the similarity and difference of those two types of cells in the role of phagocyte was estimated. Additionally, the uptake route of BLG samples by macrophages was studied by blocking each uptake route separately. The importance of a few representative receptors was evaluated by inhibition ELISA and the proinflammatory response of macrophage after uptake was evaluate by measuring few related cytokines. In Chapter 6, all result obtained from previous chapters are integrated and the modulation of the activity of macrophages and DCs by heat processed protein is discussed in an overall perspective. This includes a perspective on applications of the obtained knowledge as well as on future research. 


\section{References}

1. The chemistry of thermal food processing procedures. 2016, New York, NY: Springer Berlin Heidelberg. pages $\mathrm{cm}$.

2. Bhattacharya, S., Conventional and advanced food processing technologies. 2014, John Wiley \& Sons Inc.,: Chichester, West Sussex ; Hoboken, NJ. p. 1 online resource.

3. Trumbo, P., et al., Dietary reference intakes for energy, carbohydrate, fiber, fat, fatty acids, cholesterol, protein and amino acids. Journal of the American Dietetic Association, 2002. 102(11): p. 1621-1630.

4. Loveday, S.M., Food Proteins: Technological, Nutritional, and Sustainability Attributes of Traditional and Emerging Proteins. Annual Review of Food Science and Technology, Vol 10, 2019. 10: p. 311-339.

5. Hardy, J., M. Parmentier, and J. Fanni, Functionality of nutrients and thermal treatments of food. Proceedings of the Nutrition Society, 1999. 58(3): p. 579-585.

6. Dallas, D.C., et al., Personalizing protein nourishment. Critical Reviews in Food Science and Nutrition, 2017. 57(15): p. 3313-3331.

7. Mitchell, H.H., T.S. Hamilton, and J.R. Beadles, The Nutritional Effects of Heat on Food Proteins, with Particular Reference to Commercial Processing and Home Cooking. Journal of Nutrition, 1949. 39(3): p. 413-425.

8. Bax, M.L., et al., Cooking Temperature Is a Key Determinant of in Vitro Meat Protein Digestion Rate: Investigation of Underlying Mechanisms. Journal of Agricultural and Food Chemistry, 2012. 60(10): p. 2569-2576.

9. Korhonen, H., et al., Impact of processing on bioactive proteins and peptides. Trends in Food Science \& Technology, 1998. 9(8-9): p. 307-319.

10. Damodaran, S. and A. Paraf, Food proteins and their applications. Food science and technology. 1997, New York: Marcel Dekker. viii, 681 p.

11. Morr, C.V., Functionality of Heated Milk-Proteins in Dairy and Related Foods. Journal of Dairy Science, 1985. 68(10): p. 2773-2781.

12. Mehta, R.S., Milk Processed at Ultra-High-Temperatures - a Review. Journal of Food Protection, 1980. 43(3): p. 212-225.

13. Schmitz-Schug, I., P. Foerst, and U. Kulozik, Impact of the spray drying conditions and residence time distribution on lysine loss in spray dried infant formula. Dairy Science \& Technology, 2013. 93(4-5): p. 443-462.

14. Rudloff, S. and B. Lonnerdal, Solubility and Digestibility of Milk-Proteins in Infant Formulas Exposed to Different Heat-Treatments. Journal of Pediatric Gastroenterology and Nutrition, 1992. 15(1): p. 25-33.

15. Herczenik, E. and M.F.B.G. Gebbink, Molecular and cellular aspects of protein misfolding and disease. Faseb Journal, 2008. 22(7): p. 2115-2133.

16. Joyce, A.M., et al., Separation of the effects of denaturation and aggregation on whey-casein protein interactions during the manufacture of a model infant formula. Dairy Science \& Technology, 2017. 96(6): p. 787-806.

17. Dickinson, E. and E.L. Parkinson, Heat-induced aggregation of milk protein-stabilized emulsions: sensitivity to processing and composition. International Dairy Journal, 2004. 14(7): p. 635-645.

18. Kellow, N.J. and M.T. Coughlan, Effect of diet-derived advanced glycation end products on inflammation. Nutrition Reviews, 2015. 73(11): p. 737-759.

19. Liu, J.H., Q.M. Ru, and Y.T. Ding, Glycation a promising method for food protein modification: Physicochemical properties and structure, a review. Food Research International, 2012. 49(1): p. 170-183.

20. Laroque, D., et al., Kinetic study on the Maillard reaction. Consideration of sugar reactivity. Food Chemistry, 2008. 111(4): p. 1032-1042.

21. Goldberg, T., et al., Advanced glycoxidation end products in commonly consumed foods (vol 104, pg 1287, 2004). Journal of the American Dietetic Association, 2005. 105(4): p. 647-647. 
22. Siciliano, R.A., et al., Mass spectrometry for the analysis of protein lactosylation in milk products. Food Research International, 2013. 54(1): p. 988-1000.

23. Aalaei, K., et al., Early and advanced stages of Maillard reaction in infant formulas: Analysis of available lysine and carboxymethyl-lysine. Plos One, 2019. 14(7).

24. Drusch, S., V. Faist, and H.F. Erbersdobler, Determination of $\mathrm{N}$-epsilon-carboxymethyllysine in milk products by a modified reversed-phase HPLC method. Food Chemistry, 1999. 65(4): p. 547553.

25. Zhang, Q.B., et al., A Perspective on the Maillard Reaction and the Analysis of Protein Glycation by Mass Spectrometry: Probing the Pathogenesis of Chronic Disease. Journal of Proteome Research, 2009. 8(2): p. 754-769.

26. Sicherer, S.H. and H.A. Sampson, Food allergy. Journal of Allergy and Clinical Immunology, 2010. 125(2): p. S116-S125.

27. Sheikh, A., et al., National clinical practice guidelines for food allergy and anaphylaxis: an international assessment. Clinical and Translational Allergy, 2017. 7.

28. National Institute of Allergy and Infectious Diseases (U.S.), Food allergy : an overview. NIH publication. 2012, Bethesda, Md.: National Institute of Allergy and Infectious Diseases, U.S. Department of Health and Human Services, National Institutes of Health. 33 pages.

29. Sompayrac, L., How the immune system works. 1999, Malden, Mass.: Blackwell Science. vii, $111 \mathrm{p}$.

30. Schuijs, M.J., H. Hammad, and B.N. Lambrecht, Professional and 'Amateur' Antigen-Presenting Cells In Type 2 Immunity. Trends in Immunology, 2019. 40(1): p. 22-34.

31. Dudley, D.J., The immune system in health and disease. Baillieres Clin Obstet Gynaecol, 1992. 6(3): p. 393-416.

32. White, M.V., The Role of Histamine in Allergic Diseases. Journal of Allergy and Clinical Immunology, 1990. 86(4): p. 599-605.

33. Host, A., Cow's milk protein allergy and intolerance in infancy. Some clinical, epidemiological and immunological aspects. Pediatr Allergy Immunol, 1994. 5(5 Suppl): p. 1-36.

34. Hill, D.J., et al., The frequency of food allergy in Australia and Asia. Environmental Toxicology and Pharmacology, 1997. 4(1-2): p. 101-110.

35. Woods, R.K., et al., Prevalence of food allergies in young adults and their relationship to asthma, nasal allergies, and eczema. Annals of Allergy Asthma \& Immunology, 2002. 88(2): p. 183-189.

36. Hettinga, K., et al., The Host Defense Proteome of Human and Bovine Milk. Plos One, 2011. 6(4).

37. Hochwallner, H., et al., Cow's milk allergy: From allergens to new forms of diagnosis, therapy and prevention. Methods, 2014. 66(1): p. 22-33.

38. Machtinger, S. and R. Moss, Cow's milk allergy in breast-fed infants: the role of allergen and maternal secretory IgA antibody. J Allergy Clin Immunol, 1986. 77(2): p. 341-7.

39. Wal, J.M., An update on allergens - Cow's milk allergens. Allergy, 1998. 53(11): p. 1013-1022.

40. Jarvinen, K.M., et al., IgE and IgG binding epitopes on alpha-lactalbumin and beta-lactoglobulin in cow's milk allergy. International Archives of Allergy and Immunology, 2001. 126(2): p. 111118.

41. Bernard, H., et al., Specificity of the human IgE response to the different purified caseins in allergy to cow's milk proteins. International Archives of Allergy and Immunology, 1998. 115(3): p. 235-244.

42. Zuberbier, T., et al., Prevalence of adverse reactions to food in Germany - a population study. Allergy, 2004. 59(3): p. 338-345.

43. Morita, H., et al., Antigen-specific T-cell responses in patients with non-IgE-mediated gastrointestinal food allergy are predominantly skewed to $T(H) 2$. Journal of Allergy and Clinical Immunology, 2013. 131(2): p. 590-592.

44. Knopf, P.M., Immunomodulation and allergy. Allergy and Asthma Proceedings, 2000. 21(4): p. 215-220. 
45. Rahaman, T., T. Vasiljevic, and L. Ramchandran, Effect of processing on conformational changes of food proteins related to allergenicity. Trends in Food Science \& Technology, 2016. 49: p. 24-34.

46. Abbring, S., et al., Milk processing increases the allergenicity of cow's milk-Preclinical evidence supported by a human proof-of-concept provocation pilot. Clinical and Experimental Allergy, 2019. 49(7): p. 1013-1025.

47. Nowak-Wegrzyn, A., et al., Tolerance to extensively heated milk in children with cow's milk allergy. Journal of Allergy and Clinical Immunology, 2008. 122(2): p. 342-347.

48. Blanc, F., et al., Boiling peanut Ara $h 1$ results in the formation of aggregates with reduced allergenicity. Molecular Nutrition \& Food Research, 2011. 55(12): p. 1887-1894.

49. Madsen, C.B., et al., Risk management for food allergy. Food science and technology international series. 2014, Amsterdam ; Boston: Elsevier, AP, Academic Press is an imprint of Elsevier. xxi, 312 pages.

50. Kleber, N. and J. Hinrichs, Antigenic response of beta-lactoglobulin in thermally treated bovine skim milk and sweet whey. Milchwissenschaft-Milk Science International, 2007. 62(2): p. 121124.

51. Bu, G.H., et al., Effect of heat treatment on the antigenicity of bovine -lactalbumin and lactoglobulin in whey protein isolate. Food and Agricultural Immunology, 2009. 20(3): p. 195206.

52. Mills, E.N.C., et al., Impact of food processing on the structural and allergenic properties of food allergens. Molecular Nutrition \& Food Research, 2009. 53(8): p. 963-969.

53. Sugaya, K., et al., An aggregate-prone conformational epitope in trinucleotide repeat diseases. Neuroreport, 2003. 14(18): p. 2331-2335.

54. Davis, P.J. and S.C. Williams, Protein modification by thermal processing. Allergy, 1998. 53: $\mathrm{p}$. 102-105.

55. Zhong, J.Z., et al., Aggregation and conformational changes of bovine beta-lactoglobulin subjected to dynamic high-pressure microfluidization in relation to antigenicity. Journal of Dairy Science, 2012. 95(8): p. 4237-4245.

56. Teodorowicz, M., J. van Neerven, and H. Savelkoul, Food Processing: The Influence of the Maillard Reaction on Immunogenicity and Allergenicity of Food Proteins. Nutrients, 2017. 9(8).

57. Bu, G.H., et al., Milk processing as a tool to reduce cow's milk allergenicity: a mini-review. Dairy Science \& Technology, 2013. 93(3): p. 211-223.

58. Taheri-Kafrani, A., et al., Effects of heating and glycation of beta-lactoglobulin on its recognition by IgE of sera from cow milk allergy patients. J Agric Food Chem, 2009. 57(11): p. 4974-82.

59. Hattori, M., et al., Reduced immunogenicity of beta-lactoglobulin by conjugation with acidic oligosaccharides. Journal of Agricultural and Food Chemistry, 2004. 52(14): p. 4546-4553.

60. Kobayashi, K., et al., Modulation of the T cell response to beta-lactoglobulin by conjugation with carboxymethyl dextran. Bioconjugate Chemistry, 2003. 14(1): p. 168-176.

61. Hattori, M., et al., Reduced immunogenicity of beta-lactoglobulin by conjugation with carboxymethyl dextran. Bioconjugate Chemistry, 2000. 11(1): p. 84-93.

62. Kobayashi, K., et al., Reduced immunogenicity of beta-lactoglobulin by conjugation with carboxymethyl dextran differing in molecular weight. Journal of Agricultural and Food Chemistry, 2001. 49(2): p. 823-831.

63. Ikeda, K., et al., $\mathrm{N}$-epsilon-(carboxymethyl)lysine protein adduct is a major immunological epitope in proteins modified with advanced glycation end products of the Maillard reaction. Biochemistry, 1996. 35(24): p. 8075-8083.

64. Gebert, A., H.J. Rothkotter, and R. Pabst, $M$ cells in Peyer's patches of the intestine. International Review of Cytology - a Survey of Cell Biology, Vol 167, 1996. 167: p. 91-159.

65. Samadi, N., M. Klems, and E. Untersmayr, The role of gastrointestinal permeability in food allergy. Annals of Allergy Asthma \& Immunology, 2018. 121(2): p. 168-173. 
66. Reitsma, M., et al., Protein transport across the small intestine in food allergy. Mol Nutr Food Res, 2014. 58(1): p. 194-205.

67. Erickson, R.H. and Y.S. Kim, Digestion and Absorption of Dietary-Protein. Annual Review of Medicine, 1990. 41: p. 133-139.

68. Hur, S.J., et al., In vitro human digestion models for food applications. Food Chemistry, 2011. 125(1): p. 1-12.

69. Dupont, D., et al., Comparative resistance of food proteins to adult and infant in vitro digestion models. Molecular Nutrition \& Food Research, 2010. 54(6): p. 767-780.

70. Corzo-Martinez, M., et al., Effect of glycation on the gastrointestinal digestibility and immunoreactivity of bovine beta-lactoglobulin. International Dairy Journal, 2010. 20(11): p. 742-752.

71. Minekus, M., et al., A standardised static in vitro digestion method suitable for food - an international consensus. Food \& Function, 2014. 5(6): p. 1113-1124.

72. Singh, T.K., et al., Influence of heat and shear induced protein aggregation on the in vitro digestion rate of whey proteins. Food \& Function, 2014. 5(11): p. 2686-2698.

73. Sanz, M.L., et al., Characterization and in vitro digestibility of bovine beta-lactoglobulin glycated with galactooligosaccharides. Journal of Agricultural and Food Chemistry, 2007. 55(19): p. 7916-7925.

74. Boyer, J., D. Brown, and R.H. Liu, In vitro digestion and lactase treatment influence uptake of quercetin and quercetin glucoside by the Caco-2 cell monolayer. Nutrition Journal, 2005. 4.

75. Brodkorb, A., et al., INFOGEST static in vitro simulation of gastrointestinal food digestion. Nature Protocols, 2019. 14(4): p. 991-1014.

76. Egger, L., et al., The harmonized INFOGEST in vitro digestion method: From knowledge to action. Food Research International, 2016. 88: p. 217-225.

77. Sanchon, J., et al., Protein degradation and peptide release from milk proteins in human jejunum. Comparison with in vitro gastrointestinal simulation. Food Chemistry, 2018. 239: p. 486-494.

78. Villa, C., et al., Bovine Milk Allergens: A Comprehensive Review. Comprehensive Reviews in Food Science and Food Safety, 2018. 17(1): p. 137-164.

79. Zhou, C.Y., et al., The effect of cooking temperature on the aggregation and digestion rate of myofibrillar proteins in Jinhua ham. Journal of the Science of Food and Agriculture, 2018. 98(9): p. 3563-3570.

80. van Lieshout, G.A.A., et al., How processing may affect milk protein digestion and overall physiological outcomes: A systematic review. Critical Reviews in Food Science and Nutrition, 2019.

81. Deng, Y., et al., Effect of Maillard induced glycation on protein hydrolysis by lysine/arginine and non-lysine/arginine specific proteases. Food Hydrocolloids, 2017. 69: p. 210-219.

82. Ardura, J.A., et al., Targeting Macrophages: Friends or Foes in Disease? Frontiers in Pharmacology, 2019. 10.

83. Santiago-Schwarz, F., Dendritic cells: friend or foe in autoimmunity? Rheumatic Disease Clinics of North America, 2004. 30(1): p. 115-+.

84. Flannigan, K.L., et al., Intestinal, Antigen-Presenting Cells Key Regulators of Immune Homeostasis and Inflammation. American Journal of Pathology, 2015. 185(7): p. 1809-1819.

85. Etzerodt, A. and S.K. Moestrup, CD163 and Inflammation: Biological, Diagnostic, and Therapeutic Aspects. Antioxidants \& Redox Signaling, 2013. 18(17): p. 2352-2363.

86. Guilliams, M., et al., Dendritic cells, monocytes and macrophages: a unified nomenclature based on ontogeny. Nature Reviews Immunology, 2014. 14(8): p. 571-578.

87. Savina, A. and S. Amigorena, Phagocytosis and antigen presentation in dendritic cells. Immunological Reviews, 2007. 219: p. 143-156.

88. Bain, C.C. and A. Schridde, Origin, Differentiation, and Function of Intestinal Macrophages. Frontiers in Immunology, 2018. 9. 
89. Varol, C., E. Zigmond, and S. Jung, Securing the immune tightrope: mononuclear phagocytes in the intestinal lamina propria. Nature Reviews Immunology, 2010. 10(6): p. 415-426.

90. Sampson, H.A., et al., Mechanisms of food allergy. Journal of Allergy and Clinical Immunology, 2018. 141(1): p. 11-19.

91. Frischmeyer-Guerrerio, P.A., et al., Dendritic cell and $T$ cell responses in children with food allergy. Clinical and Experimental Allergy, 2011. 41(1): p. 61-71.

92. Grabowska, J., et al., CD169(+) Macrophages Capture and Dendritic Cells Instruct: The Interplay of the Gatekeeper and the General of the Immune System. Frontiers in Immunology, 2018. 9.

93. Kumar, S., et al., Macrophages in food allergy: An enigma. Molecular Immunology, 2013. 56(4): p. 612-618.

94. Ferenbach, D. and J. Hughes, Macrophages and dendritic cells: what is the difference? Kidney International, 2008. 74(1): p. 5-7.

95. Berger, A., Science commentary: Th1 and Th2 responses: what are they? British Medical Journal, 2000. 321(7258): p. 424-424.

96. Kiewiet, M.B.G., et al., Immunomodulating properties of protein hydrolysates for application in cow's milk allergy. Pediatric Allergy and Immunology, 2015. 26(3): p. 206-217.

97. Tsitoura, D.C. and Y. Tassios, Immunomodulation - The future cure for allergic diseases. Neuroendocrine and Immune Crosstalk, 2006. 1088: p. 100-115.

98. von Mutius, E. and D. Vercelli, Farm living: effects on childhood asthma and allergy. Nature Reviews Immunology, 2010. 10(12): p. 861-868.

99. Lluis, A., et al., Increased regulatory T-cell numbers are associated with farm milk exposure and lower atopic sensitization and asthma in childhood. Journal of Allergy and Clinical Immunology, 2014. 133(2): p. 551-+.

100. Loss, G., et al., The protective effect of farm milk consumption on childhood asthma and atopy: The GABRIELA study. Journal of Allergy and Clinical Immunology, 2011. 128(4): p. 766-U432.

101. Roth-Walter, F., et al., Pasteurization of milk proteins promotes allergic sensitization by enhancing uptake through Peyer's patches. Allergy, 2008. 63(7): p. 882-890.

102. Stojadinovic, M., et al., Cross-Linking of beta-Lactoglobulin Enhances Allergic Sensitization Through Changes in Cellular Uptake and Processing. Toxicological Sciences, 2014. 140(1): p. 224-235.

103. Jiskoot, W., et al., Mouse Models for Assessing Protein Immunogenicity: Lessons and Challenges. Journal of Pharmaceutical Sciences, 2016. 105(5): p. 1567-1575.

104. Benne, N., et al., Orchestrating immune responses: How size, shape and rigidity affect the immunogenicity of particulate vaccines. Journal of Controlled Release, 2016. 234: p. 124-134.

105. Seydoux, E., et al., Size-dependent accumulation of particles in lysosomes modulates dendritic cell function through impaired antigen degradation. International Journal of Nanomedicine, 2014. 9: p. 3885-3902.

106. Chang, T.Z., et al., Effects of ovalbumin protein nanoparticle vaccine size and coating on dendritic cell processing. Biomaterials Science, 2017. 5(2): p. 223-233.

107. Rosalia, R.A., et al., Dendritic cells process synthetic long peptides better than whole protein, improving antigen presentation and T-cell activation. European Journal of Immunology, 2013. 43(10): p. 2554-2565.

108. Thomas, C., V. Gupta, and F. Ahsan, Particle Size Influences the Immune Response Produced by Hepatitis B Vaccine Formulated in Inhalable Particles. Pharmaceutical Research, 2010. 27(5): p. 905-919.

109. Dalgediene, I., A. Luciunaite, and A. Zvirbliene, Activation of Macrophages by Oligomeric Proteins of Different Size and Origin. Mediators of Inflammation, 2018.

110. Seong, S.Y. and P. Matzinger, Hydrophobicity: an ancient damage-associated molecular pattern that initiates innate immune responses. Nature Reviews Immunology, 2004. 4(6): p. 469-478. 
111. Buongiorno, A.M., et al., Immunogenicity of advanced glycation end products in diabetic patients and in nephropathic non-diabetic patients on hemodialysis or after renal transplantation. J Endocrinol Invest, 2008. 31(6): p. 558-62.

112. Hatzioannou, A., et al., Effect of Advanced Glycation End Products on Human Thyroglobulin's Antigenicity as Identified by the Use of Sera from Patients with Hashimoto's Thyroiditis and Gestational Diabetes Mellitus. International Journal of Endocrinology, 2015.

113. Smith, P.K., et al., The false alarm hypothesis: Food allergy is associated with high dietary advanced glycation end-products and proglycating dietary sugars that mimic alarmins. Journal of Allergy and Clinical Immunology, 2017. 139(2): p. 429-437.

114. Hsu, S.C., et al., Antigen coupled with Lewis-x trisaccharides elicits potent immune responses in mice. Journal of Allergy and Clinical Immunology, 2007. 119(6): p. 1522-1528.

115. Hilmenyuk, T., et al., Effects of glycation of the model food allergen ovalbumin on antigen uptake and presentation by human dendritic cells. Immunology, 2010. 129(3): p. 437-445.

116. Ilchmann, A., et al., Glycation of a food allergen by the Maillard reaction enhances its T-cell immunogenicity: Role of macrophage scavenger receptor class A type I and II. Journal of Allergy and Clinical Immunology, 2010. 125(1): p. 175-183.

117. Buttari, B., et al., Advanced glycation end products of human beta(2) glycoprotein I modulate the maturation and function of DCs. Blood, 2011. 117(23): p. 6152-6161.

118. Mueller, G.A., et al., Identification of Maillard reaction products on peanut allergens that influence binding to the receptor for advanced glycation end products. Allergy, 2013. 68(12): p. 1546-1554.

119. Shreffler, W.G., et al., The major glycoprotein allergen from Arachis hypogaea, Ara $h$ 1, is a ligand of dendritic cell-specific ICAM-grabbing nonintegrin and acts as a Th2 adjuvant in vitro. Journal of Immunology, 2006. 177(6): p. 3677-3685. 



\section{Chapter 2}

\section{Heat treatment of $\beta$-lactoglobulin affects its survival and translocation in the upper digestive tract}

Ying Deng, Coen Govers, Monic Tomassen, Kasper Hettinga and Harry J. Wichers

Based on the manuscript submitted to Food Chemistry 
Abstract: Heat treatment is a commonly applied unit operation in the processing of $\beta$-lactoglobulin containing products. This does, however, influence its structure and thereby impacts its activity and digestibility. We describe how various heat-treatments of $\beta$-lactoglobulin change the digestibility using a modified version of the current consensus INFOGEST protocol. Additionally, protein was investigated for its translocation over the intestinal epithelial barrier, which would bring them in contact with immune cells. The extent of gastric digestibility was higher when the protein structure was more modified, while the influence of glycation with lactose was limited. Translocation studies of protein across Caco- 2 cell monolayers showed a lower translocation rate of protein heated in solution compared to the others. Our study indicates that structural modifications after different heattreatments of $\beta$-lactoglobulin increase in particular gastric digestibility and the translocation efficiency across intestinal epithelial cells, parameters that are essential for estimating the protein's interaction with immune cells. 


\section{Introduction}

Cow's milk allergy is one of the major food allergies, especially in children. The prevalence of cow's milk allergy is estimated to be $2.5 \%$ amongst children in the Western Hemisphere [1]. There is some controversy, based on methodological issues, as to whether the prevalence is increasing [2]. Cow's milk contains about $3.5 \%$ protein in which at least 25 different proteins were identified that may act as an allergen. $\beta$-Lactoglobulin (BLG), which accounts for approximately one tenth of all proteins in cow's milk, is one of the major allergens [3].

Thermal processing of milk is an essential step in the dairy industry to prolong its shelf life. However, these heat treatments may lead to unfolding, aggregation, and glycation with lactose [4]. These structure modifications can either reduce or enhance the allergenic potential of BLG depending on the processing conditions [5]. Glycation is linked to masking of epitopes and may result in reduced protein binding by IgE in cow's milk allergic patients [6]. Antigenicity of BLG was reported to increase with increasing heating temperature until $90^{\circ} \mathrm{C}$, but to decrease at even higher temperatures [7]. Moreover, thermal-processing-induced structure modifications may influence the digestibility of the protein, which could further alter its immunogenicity, as the sensitizing capacity of BLG was observed to be decreased along the digestion process [8].

Like many other food allergens, BLG resists gastric digestion, mainly due to its stability at the acidic $\mathrm{pH}$ of the gastric environment and its compact structure. This may result in increased exposure of larger fragments to the gut-associated lymphoid tissue, possibly contributing to the development of an allergic reaction [9]. The consensus in vitro digestion protocol (INFOGEST) confirmed the resistance of BLG to gastric digestion. Using this protocol, BLG was reported to be mainly digested in the intestinal phase, again reflecting physiological processes [10]. Prolonged survival of BLG was also observed in the jejunum in an in vivo study [11]. In addition to support digestion, the gastro-intestinal (GI) tract hosts a large population of immune cells and lymphoid tissue as it is an important route of pathogenic entry. It is thus essential to study the interaction between food proteins like BLG and the immune system based on a clear understanding of its digestibility, which can be influenced by the type and severity of thermal processing that has been applied. For instance, potential cleavage sites for digestive enzymes buried in the native form of a protein might be exposed due to unfolding. BLG heated at around $70^{\circ} \mathrm{C}$ in solution was reported to be susceptible to gastric digestion [12]. Contrastingly, cleavage sites could be modified because of glycation and crosslinking, which can both impair the digestibility of BLG [13, 14]. 
Allergens can be sampled from the intestinal luminal content by antigen presenting cells [15]. In addition, allergens may also actively pass through the intestinal epithelial layer and directly contact with a range of immune cells $[16,17]$. Such active transport through the epithelial layer may be studied with Caco- 2 cells that are differentiated into small intestinal-like enterocytes, which have been used widely for intestinal translocation studies [18]. Literature on the impact of thermal processing of BLG on its digestibility and intestinal epithelial passage is rather scattered, which was the reason for us to analyse these effects in one study. Such information can contribute to better evaluations of the impact of BLG processing on the intestinal immunological response.

\section{Materials and methods}

\section{Chemicals}

All chemicals were purchased from Sigma Aldrich (St Louis, Missouri, USA) unless otherwise stated.

\section{Sample preparation}

$\beta$-Lactoglobulin (BLG) was isolated from raw cow's milk as published [19] and wet-heated (W), hightemperature dry-heated $(\mathrm{H})$ or low-temperature dry-heated $(\mathrm{L})$, centrifuged, dialyzed and protein concentration measured as described in Chapter 3.

\section{In vitro gastrointestinal digestion}

The applied in vitro digestion protocol is a scaled-down adaptation of the INFOGEST model [20]. BLG samples were diluted to $2 \mathrm{mg} / \mathrm{mL}$ with $140 \mathrm{mM} \mathrm{NaCl}+5 \mathrm{mM} \mathrm{KCl}$ solution to mimic the oral situation after which we moved forward with $200 \mu \mathrm{L}$. For mimicking gastric digestion, the $\mathrm{pH}$ was set to 2 with $1 \mathrm{M} \mathrm{HCl}$ and $6.67 \mathrm{uL}$ of pepsin solution $(1092 \mathrm{U} / \mathrm{mL}$ dissolved in $0.1 \mathrm{M} \mathrm{HCl}$ ) was added. Samples were incubated for 1 hour while gently shaking at $37^{\circ} \mathrm{C}$. After the $\mathrm{pH}$ was set to 5.8 with $1 \mathrm{M} \mathrm{NaHCO}_{3}, 1.6$ $\mu \mathrm{g}$ pancreatin (6.84 $\mathrm{U} / \mathrm{mg}$ trypsin activity) and $2.36 \mu \mathrm{g} \alpha$-chymotrypsin $(65.61 \mathrm{U} / \mathrm{mg}$ ) from porcine pancreas, and bile salts ( $453.8 \mu \mathrm{g}$ sodium taurocholate and $415.0 \mu \mathrm{g}$ sodium glycodeoxycholate) dissolved together in $15 \mu \mathrm{L}$ of $0.1 \mathrm{M} \mathrm{NaHCO}_{3}$ were added. Again, the $\mathrm{pH}$ of samples was adjusted to 6.5 by $1 \mathrm{M} \mathrm{NaHCO}_{3}$ after which the samples were incubated at $37^{\circ} \mathrm{C}$ while gently shaking to mimic the intestinal digestion. As a control we prepared a digest which contained all volumes, $\mathrm{pH}$ changes and enzymes but without addition of BLG. 


\section{Sodium dodecyl sulfate polyacrylamide gel electrophoresis (SDS-PAGE)}

Non-reducing SDS-page was performed by mixing $12 \mu \mathrm{g}$ of protein sample with a corresponding volume of NuPAGE ${ }^{\circledR}$ LDS Sample Buffer (4X) (ThermoFischer, Waltham, Massachusetts, USA), followed by boiling for $5 \mathrm{~min}$ and loading on 10\% Bis-Tris gels (ThermoFischer, Waltham, Massachusetts, USA) together with a pre-stained Precision Plus Protein ${ }^{\text {TM }}$ Dual Xtra marker (Bio-Rad, Hercules, California, USA). Gels were running under $160 \mathrm{~V}$ for 50 minutes, stained by PageBlue ${ }^{\mathrm{TM}}$ Protein Staining Solution (ThermoFischer, Waltham, Massachusetts, USA) and de-stained by MilliQ water and analysed using Image Lab version 4.1 software (Bio-Rad, Hercules, California, USA). The lanes of the gels were scanned with Image Lab version 4.1 (Bio-Rad), and the percentage of undigested protein was calculated based on the band density ratio of original loading BLG samples.

\section{Ortho-phthalaldehyde (OPA) measurement}

Protein samples' free amino groups along digestion were measured by OPA as described in Chapter 3. Degree of hydrolysis ( $\mathrm{DH}, \%)$ for gastric or intestinal digestion was calculated separately by the following equation:

$$
\mathrm{DH}(\%)=\left(\frac{\# \mathrm{NH} 2 \text { after digestion }-\# \mathrm{NH} 2 \text { before digesiton }}{\# \text { peptide bonds of BLG }}\right) \times 100 \%
$$

\section{ANS measurement}

To determine the change of surface hydrophobicity during digestion, ANS measurement was applied as described in Chapter 3.

\section{Translocation of protein through the Caco-2 monolayer}

Fluorescein isothiocyanate (FITC) isomer I was used to label and track the translocation of protein through a Caco-2 monolayer. Briefly, $1 \mathrm{mg} / \mathrm{mL}$ protein sample was incubated with $0.1 \mathrm{mg} / \mathrm{mL}$ FITC in borate buffer $(10 \mathrm{mM}, \mathrm{pH} 9)$ overnight at $4{ }^{\circ} \mathrm{C}$. The unbound FITC was filtered out by passing the mixture through a NAP-10 column (GE healthcare, Chicago, Illinois, USA). The FITC labelled protein sample was eluted using $0.1 \mathrm{M} \mathrm{PBS}(\mathrm{pH} 7.4)$ at a final protein concentration of $500 \mu \mathrm{g} / \mathrm{mL}$.

Caco-2 cells were grown in a 6 or 24- well insert transwell plate (Greiner bio-one, Alphen a/d Rijn, The Netherlands) using Dulbecco's Modified Eagles Medium (DMEM) with $4.5 \mathrm{~g} / \mathrm{L}$ glucose, $4 \mathrm{mM} \mathrm{L-}$ glutamine and 25 mM HEPES (Gibco, Thermo Fisher, Waltham, Massachusetts, USA), supplemented with $10 \%$ heat inactivated fetal bovine serum (Invitrogen, Paisley, UK). Cells were seeded at a 
concentration of $0.225 \times 10^{6}$ cells $/ \mathrm{mL}$. A volume of $150 \mu \mathrm{L}$ for 24 -well and $2 \mathrm{~mL}$ for 6 -well was added to the apical side, and $750 \mu \mathrm{L}$ or $4 \mathrm{ml}$ medium was added to the basolateral side of the transwells, respectively. The cells developed into small intestinal like cells after 21 days of incubation with medium being changed three times a week.

To study BLG translocation, the apical medium of both 6 and 24-well inserts was replaced by FITC labelled protein diluted 2 times (final protein concentration $250 \mathrm{mg} / \mathrm{mL}$ ) using DMEM medium without phenol red (Gibco, Thermo Fisher, Waltham, Massachusetts, USA) or medium only as control. After 6 or 24 hours of incubation, basolateral fractions were collected. Fluorescence intensity of the basolateral medium from 24-well inserts was recorded by Infinite ${ }^{\circledR} 200$ PRO NanoQuant with i-control software (Tecan, Männedorf, Switzerland) in arbitrary units using $495 \mathrm{~nm}$ and $525 \mathrm{~nm}$ as excitation and emission wavelength. The final protein concentration was determined using a standard curve of each individual sample with a concentration range of $0-500 \mu \mathrm{g} / \mathrm{mL}$. The basolateral medium from 6well inserts was concentrated using Amicon Ultra- $0.5 \mathrm{~mL}$ Centrifugal Filters (Merck Millipore, Massachusetts, USA) with a cut-off of $3 \mathrm{kDa}$ from $4 \mathrm{ml}$ to $48 \mu \mathrm{L}$. From these concentrates, $9 \mu \mathrm{L}$ was used for SDS-PAGE as described above and the gel was scanned by Image Lab version 4.1 (Bio-Rad) using FITC detection settings.

\section{Statistics}

The statistical analysis including Dunnett's Test, $\mathrm{T}$ test and correlation analysis was performed using Prism 6 software with $p<0.05$ considered to be significant. The PCA plot was generated with the dataset of variables being mean centred and weighted by 1 /standard deviation by Unscrambler software (CAMO, Oslo, Norway).

\section{Results}

\section{Wet-heated BLG was digested much faster in vitro than native or high- or low-temperature dry-heated BLG}

Following heat treatment in the presence or absence of lactose, we observed a minimal effect of lowtemperature dry-heating on the polymerisation degree of BLG and the strongest effect of wet-heating, independent of the presence of lactose (Figure 2.1). An intermediate effect on polymerisation of hightemperature dry-heating was observed, but unlike for the other treatments, polymerisation of BLG was enhanced by the presence of lactose. After heating, we digested the BLG samples in a two-step (gastric and intestinal) in vitro digestion model. SDS-PAGE revealed that the monomeric (18 kDa) and 
dimeric ( $36 \mathrm{kDa}$ ) bands of native BLG remained intact after the gastric phase. Similar to native BLG, the gastric digestion of low-temperature dry-heated BLG was very limited. High-temperature dry-heated BLG appeared to be digested somewhat more than both these samples in the gastric phase. Contrastingly, a considerably larger proportion of wet-heated BLG appeared to be digested in the gastric phase, as large aggregates were no longer visible. For none of the heat treatments, the presence of lactose appeared to affect the degradation of BLG during the gastric phase.

A quantitative analysis of the observed protein bands using density scanning revealed that for native BLG approximately $96 \%$ of protein remained undigested following gastric digestion (Table 2.1). Despite that high- and low-temperature dry-heated samples demonstrated a similar digestion pattern compared to native BLG as described above, these treatments did result in a significant higher gastric digestion, with undigested protein in these samples ranging between $63 \%$ and $75 \%$. Wet-heated BLG samples were digested most extensively in the gastric phase, with only about $38 \%$ undigested protein remaining detectable.

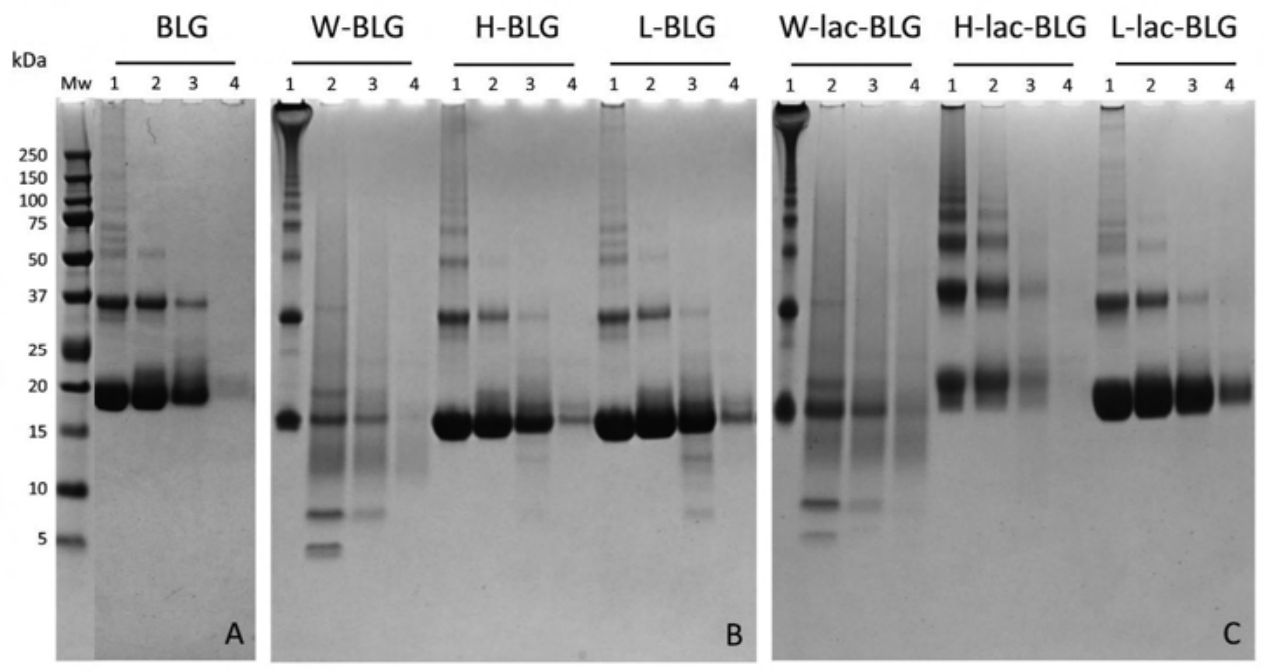

Figure 2.1 Native and dry-heated BLG were hardly digested in the gastric phase, but wet-heating prior to digestion resulted in considerably higher gastric digestion. Native BLG (A), wet-heated, high- and low-temperature dry-heated BLG in the absence (B) or presence of lactose $(C)$ were loaded on SDS-PAGE as lane 1: before digestion; lane 2: following gastric digestion; lane 3: following 10 min of intestinal digestion; lane 4: following 120 min of intestinal digestion; Mw: molecular weight marker. 
Table 2.1 Percentage of native and heated BLG detectable with SDS-Page following various steps of digestion.

\begin{tabular}{llllllll}
\hline Protein Amount (\%) & BLG & W-BLG & H-BLG & L-BLG & W-lac-BLG & H-lac-BLG & L-lac-BLG \\
\hline 60 min gastric & $95.9 \pm 3.1$ & $38.7 \pm 5.8^{* * *}$ & $63.5 \pm 3.3^{* * *}$ & $74.6 \pm 7.0^{* *}$ & $38.1 \pm 8.5^{* * *}$ & $62.7 \pm 6.5^{* * *}$ & $66.9 \pm 0.4^{* * *}$ \\
10 min intestinal & $77.0 \pm 2.0$ & $5.7 \pm 2.8^{* * *}$ & $36.0 \pm 4.9^{* * *}$ & $58.8 \pm 6.4^{* *}$ & $15.2 \pm 1.8^{* * *}$ & $19.8 \pm 3.1^{* * *}$ & $56.3 \pm 3.1^{* * *}$ \\
120 min intestinal & $4.5 \pm 2.6$ & $0.4 \pm 0.5$ & $5.4 \pm 0.6$ & $6.7 \pm 0.6$ & $3.0 \pm 2.3$ & $2.8 \pm 2.0$ & $10.5 \pm 2.5^{*}$ \\
\hline
\end{tabular}

Note: $B L G$ was untreated or heated $(W, L$ or $H)$ in absence or presence of lactose. The shown value was averaged based on 3 independent experiments data \pm standard deviation and the statistical differences were calculated with Dunnett's Test compared each heated BLG sample to native BLG after every digestion step. ${ }^{*} p<0.05 ;{ }^{* *} p<0.01 ;{ }^{* *} p<0.001$.

During the intestinal digestion, the samples analysed after 10 minutes demonstrated that undigested protein, albeit already at a lower intensity, was still clearly visible (Figure 2.1). Surprisingly, digestion of BLG that was dry-heated in the absence of lactose resulted in the presence of BLG fractions smaller than the monomeric BLG. After 120 min of intestinal digestion, native BLG was almost completely degraded with only a faint monomeric BLG band remaining. We observed a similar impact of intestinal digestion for all the other samples. The remaining band of undigested BLG was the clearest for the lowtemperature dry-heated BLG in the presence of lactose.

Quantitative analysis confirmed the presence of undigested BLG in samples after ten minutes of intestinal digestion (Table 2.1). Although a clearly reduced amount of undigested protein was observed in all samples, this was most pronounced for wet-heated BLG of which only 6 and 15\% was still detectable in the absence and presence of lactose, respectively. After completing the digestion protocol, all BLG samples were almost completely digested, independent of treatment, as the percentage of remaining undigested protein for all samples was below $10.5 \%$ and only the low temperature dry heated BLG was higher compared to native BLG.

\section{Relationship between gastric digestibility and physicochemical properties of BLG samples}

The physicochemical properties of the heat-treated BLG samples were investigated completely in Chapter 3. Here, we analysed whether there is a relation between these properties and the observed differences in gastric digestibility for these samples. A PCA plot (Figure 2.2) and a correlation analysis (Figure 2.S1) of the physicochemical properties of the variously processed BLG samples versus the gastric digestibility (percentage of digested protein) showed that the proportion of polymers, exposure 
of hydrophobic regions and percentage of $\alpha$-helix were positively related to gastric digestibility, as opposed to $\beta$-sheet structure and the proportion of monomers that were negatively related.

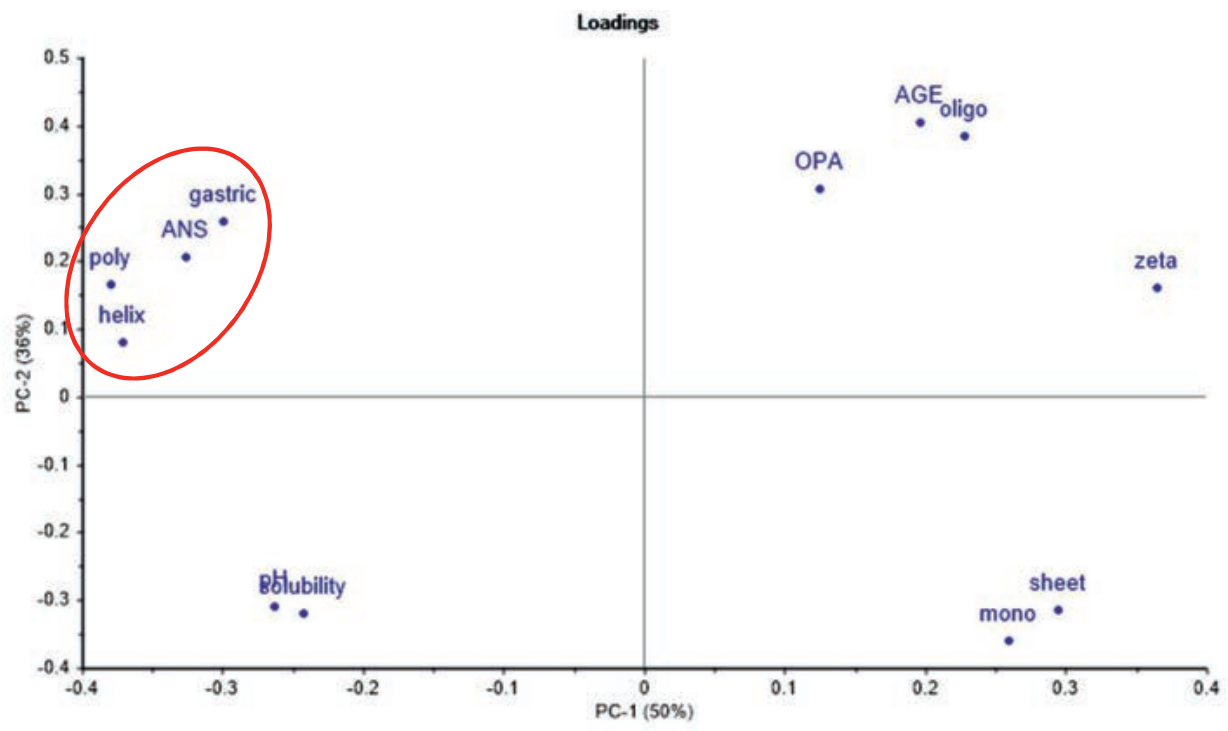

Figure 2.2 Gastric digestibility of BLG samples was related to sample hydrophobicity, proportion of polymers and percentage of $\alpha$-helix. Untreated or heated (W, L or H) BLG in absence or presence of lactose and relationships between their degree of digestion in the gastric phase (gastric), solubility, $\mathrm{pH}$, AGE formation (AGE), glycation (OPA), percentage of $\alpha$-helix (helix) or B-sheet (sheet), proportion of monomer and dimer (mono), oligomers (oligo), polymers (poly), exposure of hydrophobic regions (ANS) and surface charge (zeta) were investigated using a PCA.

\section{Wet-heated BLG samples were more extensively hydrolysed during gastric digestion compared to other samples}

The degree of protein hydrolysis during digestion can be quantified by the percentage of released free amino groups relative to the total number of peptide bonds (161 for BLG). As shown in Figure 2.3, the degree of hydrolysis (DH) in the gastric phase for native and dry-heated BLG samples was lower (around 1\%) than for wet-heated BLG samples. For wet-heated BLG, the presence of lactose seemed to increase the $\mathrm{DH}$ to a value close to $5 \%$ which is significantly higher than for the native and dryheated BLG samples. Hydrolysis of the native and dry-heated BLG samples occurred mainly during the intestinal phase, with the DH being over $6 \%$. Interestingly, the presence of lactose in the heating process seemed to exhibit an inhibitory effect during the intestinal digestion phase, especially for wetheated samples, leading to a significantly lower $\mathrm{DH}$ value. 
A

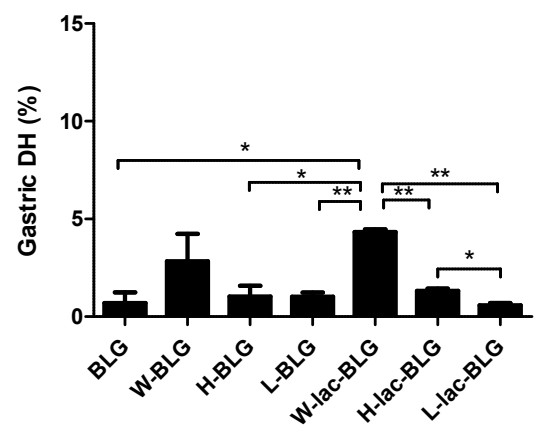

B

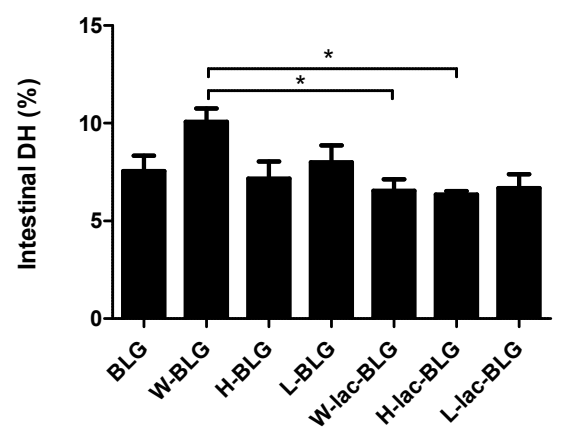

Figure 2.3 Wet-heated BLG was most efficiently digested and the presence of lactose specifically inhibited intestinal digestion. BLG was untreated or heated $(W, L$ or $H)$ in absence or presence of lactose. Degree of hydrolysis for gastric $(A)$ and intestinal digestion (B) was calculated per digestion phase separately and shown in a bar chart with $N=2$ independent assays. Statistical differences were calculated with two-tailed unpaired $T$ test between any two samples: ${ }^{*} p<0.05 ;{ }^{*} p<0.01$.

During gastric digestion, hydrophobic regions in wet-heated BLG samples processed in the presence of lactose were lost

Because of the importance of hydrophobicity as a marker for a protein's structural integrity and its importance in uptake of processed BLG by THP-1 macrophages (Chapter 3), changes in hydrophobicity of the samples were measured during digestion. For all samples, the ANS signal decreased during digestion (Figure 2.4). Before digestion, the ANS signal for wet-heated BLG samples was significantly higher than for the other samples. The ANS signal of wet-heated BLG in the absence of lactose remained significantly higher compared to native BLG until $10 \mathrm{~min}$ of intestinal digestion and remained numerically, but not significantly, higher after completing the intestinal digestion. On the contrary, we observed a strong decrease in hydrophobicity of wet-heated BLG in the presence of lactose during the gastric phase to a value close to native and dry-heated BLG samples, reaching the same level after the entire digestion process. 

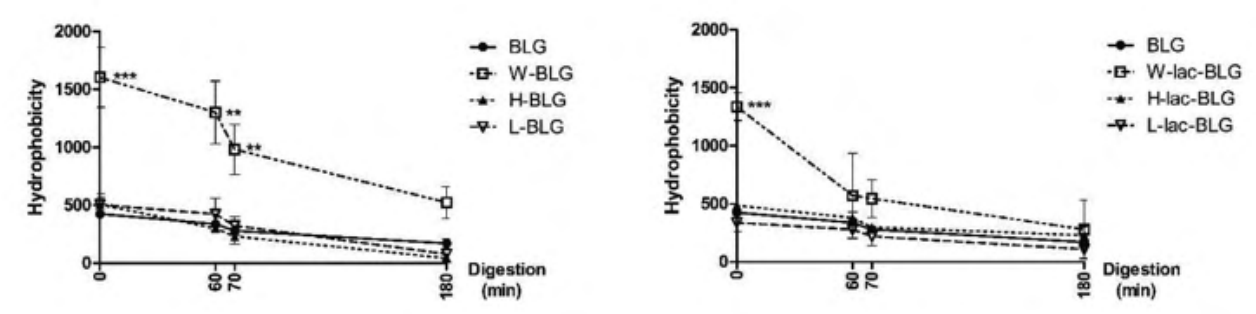

Figure 2.4 Hydrophobicity of wet-heated BLG in the presence of lactose decreased significantly upon gastric digestion. $B L G$ was untreated or heated $(W, L$ or $H)$ in absence or presence of lactose and the data were collected from 2 independent experiments with statistical differences calculated with Dunnett's Test compared processed to native BLG at each digestion step: ${ }^{* *} p<0.01 ;{ }^{* * *} p<0.001$.

\section{Wet-heated BLG was less likely to pass across a Caco-2 epithelial monolayer}

More than half of the native BLG and dry-heated BLG samples, as well as a considerable proportion of wet-heated BLG (> 38\%), remained undigested after the gastric digestion (Table 2.1). Hence, part of the BLG in the samples will enter the small intestine in its undigested form. To understand the passage of undigested protein across epithelial cells, we tested for translocation of BLG across Caco-2 monolayers. To this end, we FITC-labelled BLG samples and applied them for 6 or 24 hours to 3 weeks old transwell-grown small intestinal-like Caco-2 cells. The amount of protein transferred from the apical to the basolateral side was determined by measuring the fluorescent signal of the basolateral fraction (Figure 2.5A). Slightly less than $0.8 \%$ of total input fluorescence signal of native BLG was detectable at the basolateral side after 6 hours of incubation. Translocation of wet-heated BLG in presence or absence of lactose to the basolateral side was significantly less compared to native BLG (ca. 0.1 and $0.3 \%$, respectively). All other samples showed translocation across the Caco-2 monolayer similar to native BLG.

To identify the protein sizes that translocated to the basolateral compartments, we performed SDSPAGE with concentrated samples. Due to the limited effect from the absence of lactose, we focused on the concentrated basolateral fractions for native BLG and lactose-containing samples only (Figure 2.5B). SDS-PAGE analysis showed that the translocated FITC signal mainly corresponds to monomeric BLG in all samples. For the samples with a relative high translocation rate (Figure 2.5A) and strong monomeric BLG signal (Figure 2.5B left), i.e. native and low-temperature dry-heated BLG in the presence of lactose, there were clear fluorescent bands detectable in basolateral medium after 6 hours 
of incubation (Figure 2.5B right). Moreover, the intensity of the protein fluorescent bands increased for all samples upon longer incubation time with the Caco-2 cells, indicating continued translocation.

A

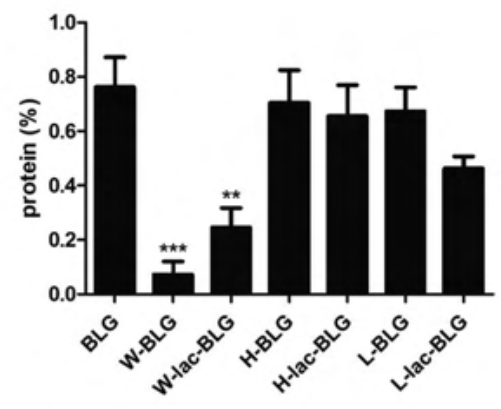

B

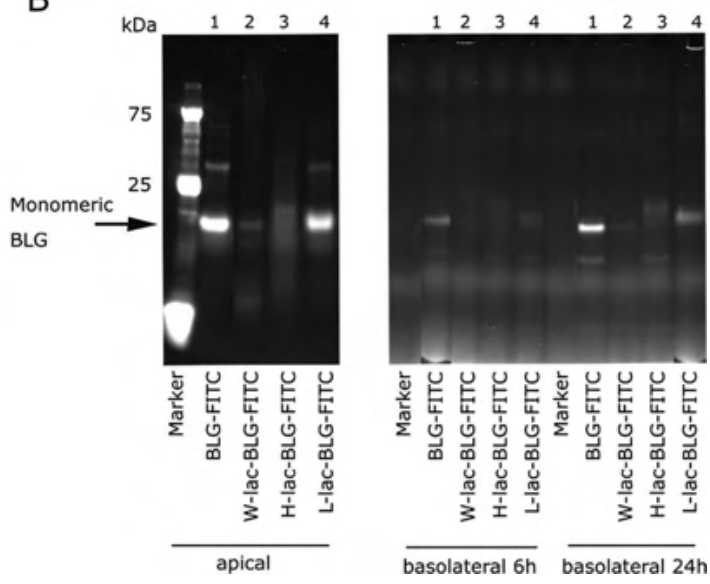

Figure 2.5 Translocation of wet-heated BLG was significantly lower than the other samples. BLG was untreated or heated (W, $H$ or $L)$ in the presence of lactose, labelled with FITC and added to the apical side of a Caco-2 transwell insert. (A) After 6 hours incubation, protein percentage was calculated based on the protein amount corresponding to the detected fluorescent signal of basolateral compartment. Cell experiments were repeated 3-4 times based on two parallel FITC labelled sample sets. The shown value was averaged \pm standard deviation and the statistical differences were calculated with Dunnett's Test compared to BLG: ${ }^{* *} p<0.01 ;{ }^{* *} p<0.001$. (B) Fluorescence scans were made of the SDS-PAGE gel of the samples and a representative figure is shown of samples added to the apical side (left) and the concentrated basolateral fractions resulting from 6 and 24 hours of incubation (right).

\section{Discussion}

The native structure of BLG is made up of nine anti-parallel $\beta$-sheets and one $\alpha$-helix [21], making it rather compact and resistant to pepsinolysis in the gastric phase and partially resistant to degradation by trypsin in the duodenal phase [22]. Although several pepsin cleavage sites have been predicted in the amino acid sequence of BLG, the integrity of the $\beta$-barrel structure under acid conditions [23] protects the protein from hydrolysis by rendering the potential cleavage sites inaccessible to this enzyme [24]. Literature reporting that BLG is hardly digested in the gastric compartment but mainly in the intestine [10] are in line with our findings (Figure 2.1 and Table 2.1), thereby validating the applied in vitro digestion protocol. Building on this, we studied a range of well-defined BLG processing 
conditions, to get a better understanding of the relation between the changes to BLG induced by processing and its digestibility.

Heating BLG in a watery solution has previously been observed to accelerate pepsin digestion [25, 26]. BLG heated under this condition was reported to exhibit increased flexibility of its tertiary structure and conformational changes [27], as well as unfolding of the protein, leading to the formation of aggregates [28]. With these structural changes, higher exposure of hydrophobic structures was observed by us (Figure 2.3) and also others [29, 30]. The hydrophobic binding sites are involved in the hydrolysis by pepsin [31,32], thus enhanced accessibility of peptic cleavage sites would be expected in wet-heated BLG. Indeed, we observed a loss of hydrophobic regions upon gastric digestion, and this decrease of hydrophobicity was related to the degree of hydrolysis, as shown by wet-heated BLG in Figure 2.3A and 2.4. The correlation between structural changes and gastric digestibility is indicated by Figure 2.2 and 2.S1, which show a clear relation between gastric digestibility and the structural change indicators-secondary structure, aggregation, and hydrophobicity. This is in line with our observation of increasing gastric digestibility correlating with the extent of structural modifications from native to low-temperature dry-heated, high-temperature dry-heated and wet-heated BLG as reported in Chapter 3. Higher peptic hydrolysis was observed for BLG samples heated under identical conditions in the presence of lactose, which is probably due to increased conformational changes induced by glycation $[33,34]$.

Not all previous studies on in vitro BLG intestinal digestion report similar results. For example, no significant digestion was observed in a study [13], while in other studies, BLG could be partly digested by trypsin (up to $30 \%$ ) or even completely digested by the end of intestinal digestion [22]. The variability in digestion protocols and activity of the used enzymes may have led to these different results. We observed complete digestion of all BLG samples after two hours of intestinal digestion (Figure 2.1 and Table 2.1). The lower digestibility observed for intestinal digestion of the lactosecontaining heated samples, especially in wet-heated BLG, may be explained by modification of cleavage sites as the amino acids involved in glycation, lysine and arginine, are also cleavage sites for trypsin. This is in agreement with a previous study reporting that an increased degree of glycation increases a protein's resistance to hydrolysis by lysine/arginine specific proteases [35]. Undigested BLG was found in the jejunum one hour (and in some cases even two hours) after oral ingestion in vivo [11]. In our study, the existence of undigested protein after 10 minutes of intestinal digestion was also observed. Due to this presence of undigested protein in the small intestine, the interaction between undigested BLG samples with intestinal cells is physiologically relevant. 
Translocation of BLG over the intestinal epithelial barrier can lead to the exposure of BLG to immune cells, which, in particular for heat-treated BLG, may lead to immune activation (Chapter 3). Translocation over intestinal epithelial cells can occur either paracellular or transcellular and the latter was reported to be the main route for BLG translocation [36]. Wet-heated BLG showed the lowest level of translocation through the Caco-2 layer (Figure 2.5), which was also observed by another study [37], although they did not study the effects of glycation in detail. The significant differences between the translocation of native and wet-heated BLG samples may be related to the formation of aggregates and structural modifications. A decrease in transport of particles through a Caco-2 monolayer was previously related to increasing particle size [38]. Moreover, it was also observed that hydrophobic compounds had a lower transport rate across Caco-2 monolayer compared to hydrophilic compounds [39]. Thus, the high hydrophobicity of wet-heated BLG might have further decreased its translocation through the Caco-2 monolayer.

\section{Conclusion}

As shown in our study, native BLG resists in vitro gastric and early intestinal digestion. Heat processing increased its digestibility to different extents, depending on the specific heating conditions. However, the undigested form of all processed samples still existed after 10 minutes of intestinal digestion. Furthermore, we demonstrated that undigested BLG was able to pass over an intestinal epithelial cell layer. This would allow the native or processed BLG to interact with local immune cells. Previously we demonstrated that wet-heating of BLG most strongly increased uptake by macrophages. However, here we demonstrate that wet-heated BLG was the most efficiently digested and also minimally translocated over the intestinal epithelial cells. We confirmed that heat-treatment affects BLG's digestibility and translocation over intestinal epithelial cells. Taken together with the findings in Chapter 3, this indicates that BLG processing conditions and further in vivo analysis should be considered as there is a potential for low grade chronic immune activation by heat-treated BLG.

\section{Acknowledgments}

The authors thank Yankang Li for some pilot experiments. 


\section{References}

1. Sicherer, S.H. and H.A. Sampson, Food allergy. Journal of Allergy and Clinical Immunology, 2010. 125(2): p. S116-S125.

2. Flom, J.D. and S.H. Sicherer, Epidemiology of Cow's Milk Allergy. Nutrients, 2019. 11(5).

3. Savilahti, E. and M. Kuitunen, Allergenicity of cow milk proteins. J Pediatr, 1992. 121(5 Pt 2): p. S12-20.

4. Morr, C.V., Functionality of Heated Milk-Proteins in Dairy and Related Foods. Journal of Dairy Science, 1985. 68(10): p. 2773-2781.

5. Rahaman, T., T. Vasiljevic, and L. Ramchandran, Effect of processing on conformational changes of food proteins related to allergenicity. Trends in Food Science \& Technology, 2016. 49: p. 24-34.

6. Taheri-Kafrani, A., et al., Effects of heating and glycation of beta-lactoglobulin on its recognition by IgE of sera from cow milk allergy patients. J Agric Food Chem, 2009. 57(11): p. 4974-82.

7. $\mathrm{Bu}, \mathrm{G} . \mathrm{H}$. , et al., Effect of heat treatment on the antigenicity of bovine -lactalbumin and lactoglobulin in whey protein isolate. Food and Agricultural Immunology, 2009. 20(3): p. 195206.

8. Bogh, K.L., V. Barkholt, and C.B. Madsen, The Sensitising Capacity of Intact beta-Lactoglobulin Is Reduced by Co-Administration with Digested beta-Lactoglobulin. International Archives of Allergy and Immunology, 2013. 161(1): p. 21-36.

9. Moreno, F.J., Gastrointestinal digestion of food allergens: Effect on their allergenicity. Biomedicine \& Pharmacotherapy, 2007. 61(1): p. 50-60.

10. Egger, L., et al., The harmonized INFOGEST in vitro digestion method: From knowledge to action. Food Research International, 2016. 88: p. 217-225.

11. Sanchon, J., et al., Protein degradation and peptide release from milk proteins in human jejunum. Comparison with in vitro gastrointestinal simulation. Food Chemistry, 2018. 239: p. 486-494.

12. Macierzanka, A., et al., The effect of gel structure on the kinetics of simulated gastrointestinal digestion of bovine beta-lactoglobulin. Food Chemistry, 2012. 134(4): p. 2156-2163.

13. Pinto, M.S., et al., Heating and glycation of beta-lactoglobulin and beta-casein: Aggregation and in vitro digestion. Food Research International, 2014. 55: p. 70-76.

14. Corzo-Martinez, M., et al., Effect of glycation on the gastrointestinal digestibility and immunoreactivity of bovine beta-lactoglobulin. International Dairy Journal, 2010. 20(11): p. 742-752.

15. Farache, J., et al., Luminal Bacteria Recruit CD103(+) Dendritic Cells into the Intestinal Epithelium to Sample Bacterial Antigens for Presentation. Immunity, 2013. 38(3): p. 581-595.

16. Price, D., L. Ackland, and C. Suphioglu, Nuts ' $n$ ' guts: transport of food allergens across the intestinal epithelium. Asia Pac Allergy, 2013. 3(4): p. 257-65.

17. Hepworth, M.R., et al., Innate lymphoid cells regulate CD4(+) T-cell responses to intestinal commensal bacteria. Nature, 2013. 498(7452): p. 113-+.

18. Bodinier, M., et al., Intestinal translocation capabilities of wheat allergens using the caco- 2 cell line. Journal of Agricultural and Food Chemistry, 2007. 55(11): p. 4576-4583.

19. de Jongh, H.H.J., T. Groneveld, and J. de Groot, Mild isolation procedure discloses new protein structural properties of beta-lactoglobulin. Journal of Dairy Science, 2001. 84(3): p. 562-571.

20. Alegria, A., G. Garcia-Llatas, and A. Cilla, Static Digestion Models: General Introduction, in The Impact of Food Bioactives on Health: in vitro and ex vivo models, K. Verhoeckx, et al., Editors. 2015: Cham (CH). p. 3-12.

21. Hamada, D., S. Segawa, and Y. Goto, Non-native alpha-helical intermediate in the refolding of beta-lactoglobulin, a predominantly beta-sheet protein. Nature Structural Biology, 1996. 3(10): p. 868-873. 
22. Dupont, D., et al., Comparative resistance of food proteins to adult and infant in vitro digestion models. Molecular Nutrition \& Food Research, 2010. 54(6): p. 767-780.

23. Mahe, S., et al., Digestion of Bovine-Milk Proteins in Patients with a High Jejunostomy. American Journal of Clinical Nutrition, 1991. 54(3): p. 534-538.

24. Guo, M.R., et al., Susceptibility of beta-lactoglobulin and sodium caseinate to proteolysis by pepsin and trypsin. Journal of Dairy Science, 1995. 78(11): p. 2336-2344.

25. Kitabatake, N. and Y.I. Kinekawa, Digestibility of bovine milk whey protein and betalactoglobulin in vitro and in vivo. Journal of Agricultural and Food Chemistry, 1998. 46(12): $p$. 4917-4923.

26. Peram, M.R., et al., In vitro gastric digestion of heat-induced aggregates of beta-lactoglobulin. Journal of Dairy Science, 2013. 96(1): p. 63-74.

27. Seo, J.A., et al., Thermal denaturation of beta-lactoglobulin and stabilization mechanism by trehalose analyzed from Raman spectroscopy investigations. J Phys Chem B, 2010. 114(19): p. 6675-84.

28. Peixoto, P.D.S., et al., Formation of beta-Lactoglobulin Aggregates from Quite, Unfolded Conformations upon Heat Activation. Langmuir, 2019. 35(2): p. 446-452.

29. Creamer, L.K., et al., Heat-induced redistribution of disulfide bonds in milk proteins. 1. Bovine beta-lactoglobulin. Journal of Agricultural and Food Chemistry, 2004. 52(25): p. 7660-7668.

30. Havea, P., A.J. Carr, and L.K. Creamer, The roles of disulphide and non-covalent bonding in the functional properties of heat-induced whey protein gels. Journal of Dairy Research, 2004. 71(3): p. 330-339.

31. Tang, J., Specificity of Pepsin and Its Dependence on a Possible Hydrophobic Binding Site. Nature, 1963. 199(489): p. 1094-1095.

32. Ahn, J., et al., Accessing the reproducibility and specificity of pepsin and other aspartic proteases. Biochimica Et Biophysica Acta-Proteins and Proteomics, 2013. 1834(6): p. $1222-$ 1229.

33. Morgan, F., et al., Glycation of bovine beta-Lactoglobulin: effect on the protein structure. International Journal of Food Science and Technology, 1999. 34(5-6): p. 429-435.

34. Chevalier, F., et al., Maillard glycation of beta-lactoglobulin induces conformation changes. Nahrung-Food, 2002. 46(2): p. 58-63.

35. Deng, Y., et al., Effect of Maillard induced glycation on protein hydrolysis by lysine/arginine and non-lysine/arginine specific proteases. Food Hydrocolloids, 2017. 69: p. 210-219.

36. Roth-Walter, F., et al., Pasteurization of milk proteins promotes allergic sensitization by enhancing uptake through Peyer's patches. Allergy, 2008. 63(7): p. 882-890.

37. Perusko, M., et al., Glycation of the Major Milk Allergen beta-Lactoglobulin Changes Its Allergenicity by Alterations in Cellular Uptake and Degradation. Molecular Nutrition \& Food Research, 2018. 62(17).

38. Derakhshandeh, K., G. Hochhaus, and S. Dadashzadeh, In-vitro Cellular Uptake and Transport Study of 9-Nitrocamptothecin PLGA Nanoparticles Across Caco-2 Cell Monolayer Model. Iranian Journal of Pharmaceutical Research, 2011. 10(3): p. 425-434.

39. Wirth, M., E. Bogner, and F. Gabor, Parameters influencing the transport rate of marker compounds across Caco-2 monolayers. Scientia Pharmazeutica, 2001. 69: p. 91-104. 


\section{Supplementary data}

A

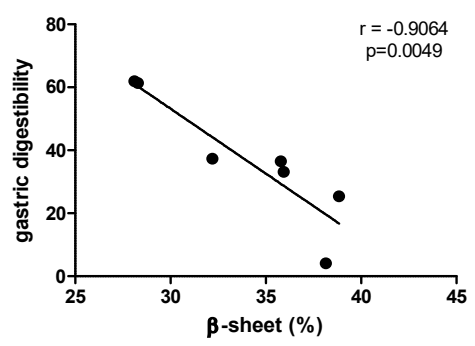

C

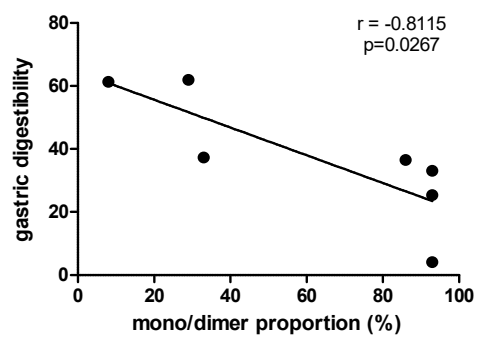

$E$

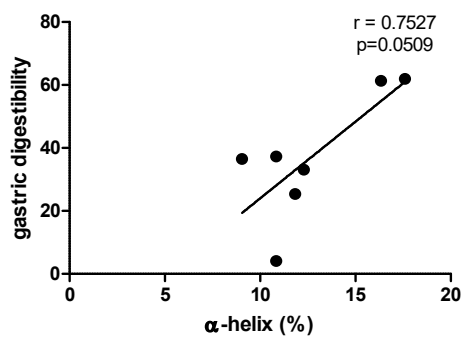

B

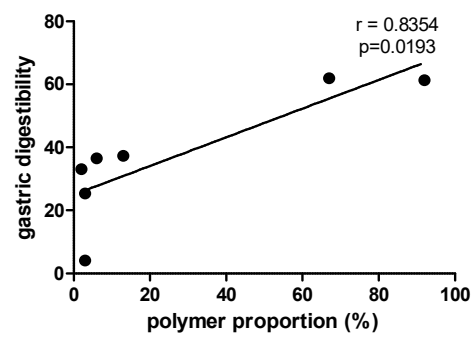

D

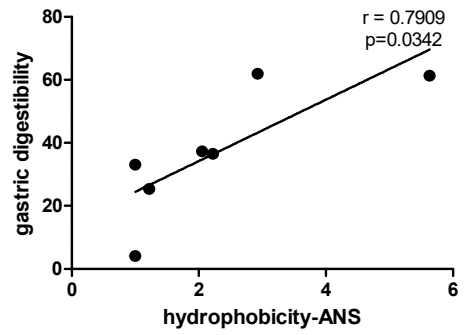

Figure 2.S1 Gastric digestibility of BLG samples correlates with secondary structure, aggregation and hydrophobicity. Percentage of protein digested during gastric phase was plotted against B-sheet, polymer and monomer/dimer proportion, exposure of hydrophobic region and $\alpha$-helix. The correlations were strong with $|r|>0.75$ and significant with $p \leq 0.05$. 



\section{Chapter 3}

Hydrophobicity and aggregation, but not glycation, are key determinants for uptake of thermally processed $\beta$-lactoglobulin by THP-1 macrophages

Ying Deng, Coen Govers, Shanna Bastiaan-Net, Nina van der Hulst, Kasper Hettinga and Harry J. Wichers

Based on the article published in Food Research International (2019) 120, 102-173 
Abstract: The aim of this study is to investigate the immunological relevance of modifications of food protein structure caused by thermal processing. We investigated the uptake of $\beta$-lactoglobulin, treated with 3 different processing methods, by THP-1 macrophages: wet heating $\left(60^{\circ} \mathrm{C}\right.$ in solution) and highor low-temperature $\left(130^{\circ} \mathrm{C}\right.$ or $50{ }^{\circ} \mathrm{C}$, respectively) dry heating, combined with either of 8 types of saccharides or without saccharide. The processing method that was applied significantly affected the uptake of $\beta$-lactoglobulin by THP-1 macrophages, while the type of saccharide only had an influence in high-temperature dry heated samples. A set of physicochemical parameters of processed samples was determined, to determine the samples' molecular weight, hydrophobicity, amyloid-like structure, surface charge and secondary structure. Analysis of protein structure alterations indicated the uptake to be linked to the wet heating processing method and percentage of $\alpha$-helix structure, amyloid-like structures, polymers, and hydrophobicity. We hypothesize that both amyloid-like structures and molecular weight were related to the increased hydrophobicity and therefore postulate that the exposure of hydrophobic regions is the leading physicochemical characteristic for the observed uptake of wet heated BLG samples by THP-1 macrophages. This work demonstrates how differential thermal processing of foods, through protein modification, can have an impact on its interactions with the immune system. 


\section{Introduction}

Bovine milk is a common component of the human diet, especially in western countries. It contains nutrients such as lactose, fat, oligosaccharides, minerals and proteins. Heat treatments like pasteurization and ultra-high temperature (UHT) are widely used to increase the safety of raw milk and extend its shelf life. Such thermal treatments will also lead to structural changes of the milk proteins. In fact, protein aggregation and glycation were observed as a consequence of the usage of high temperature in the production of infant formula [1]. An intriguing question is whether such heat modifications could impact the development of the, still maturing, infant's immune system.

During food processing, protein is often heat-treated in the presence of saccharides, with which it can chemically react. This reaction, called the Maillard reaction or glycation, leads to modifications of protein structure, ranging from the formation of Amadori products in an early stage to protein crosslinking and degradation in advanced stages of glycation [2]. Heating processes in the presence of saccharides and the structural changes caused by this heating were reported to affect the immunological properties of proteins. For instance, glycation of ovalbumin enhanced its uptake by dendritic cells and its immunogenicity compared to the native protein [3, 4]. Dry heating of whey protein at $130^{\circ} \mathrm{C}$ in the presence of lactose led to intense glycation with generation of higher particle diameter and sulfhydryl group accessibility. As a result, the glycated whey protein demonstrated an increased affinity for the receptor for advanced glycation end products (RAGE), and anti-whey IgG binding was reduced [5]. Heat denaturation of the globular whey protein $\beta$-lactoglobulin (BLG), the major protein component of whey protein, was also observed to induce increased infiltration of mononuclear inflammatory cells and eosinophils in the gastro-intestinal mucosa and a lowered IgE response compared to native BLG, in an animal trial [6]. Also, the binding of rabbit antiserum to BLG was shown to depend on the intensity of its prior heat treatment [7].

To clearly link protein structural properties with immunogenicity, BLG represents an interesting and versatile model, as it is a bovine-milk-derived protein, which is consumed in various processed forms, from mainly native in pasteurized dairy products to denatured and glycated in high-temperature dried products. BLG is one of the major allergens in cow's milk [8]. It consists of 162 amino acids including 16 lysine residues, 3 arginine's and 1 free cysteine residue [9], which all allow for irreversible tertiary structure changes such as aggregation upon heat treatment and glycation when this is done in presence of reducing saccharides [10-13].

In this study we applied three different heat-treatments to BLG in the presence, or absence, of various reducing saccharides. The nature of the saccharide used for BLG glycation was reported to determine 
the type and extent of glycation-induced changes potentially resulting in differences in physiochemical properties of BLG [14]. Dry heating at $130{ }^{\circ} \mathrm{C}$ will severely change the protein structure and induces aggregation and glycation as well as lead to the formation of advanced glycation end products [15]. A mild in-solution heating process for a prolonged time is a common alternative method to induce limited glycation $[16,17]$, where the potential formation of aggregates with this method is often overlooked. Finally, heating below the denaturation temperature of BLG in dry form limits the structural changes of the protein itself [18] and induces only the early stage of glycation. The resulting glycated and/or aggregated BLG samples were analyzed for their physicochemical properties, which were then related to their uptake by THP-1-derived macrophage immune cells.

\section{Materials and methods}

\section{Chemicals}

All chemicals were purchased from Sigma Aldrich (St Louis, Missouri, USA) unless otherwise stated.

\section{BLG purification}

Raw cow's milk was collected at the Research Facility 'Carus' of the Department of Animal Sciences, Wageningen UR. $\beta$-Lactoglobulin (BLG) was isolated from raw cow's milk by a mild anion exchange gel DEAE Sepharose C-6B (GE healthcare, Chicago, Illinois, USA) purification procedure described by De Jongh et al. [19]. Isolated BLG was lyophilised (Alpha 1-4 LD, Christ, Osterode, Germany) and stored at $-20{ }^{\circ} \mathrm{C}$. The resulting native BLG content was higher than $94 \%$ according to size exclusion chromatography.

\section{Sample preparation}

\section{Heat processing of BLG}

Purified BLG was dissolved in sodium phosphate buffer (10 mM, pH 7.4, same below) to a protein concentration of $13 \mathrm{mg} / \mathrm{mL}$. To this BLG solution, eight different types of saccharides were added which were D-glucose, D-galactose, D-fructose, D-lactose, D-maltose, maltodextrin (dextrose equivalent 16.5-19.5), fructo-oligosaccharide (FOS; Nutricia Research, Utrecht, the Netherlands), and galacto-oligosaccharide (GOS; Royal FrieslandCampina, Wageningen, the Netherlands) in a molar ratio of 1:80 (protein:saccharide) which is similar to raw milk, corresponding to 4 reducing saccharide ends versus 1 free amino group or no saccharide (control). Solutions of BLG and saccharides were treated as follows: 1 . High-temperature dry heating $(\mathrm{H})$ : according to the protocol described by Liu et al. [20]. The sample solutions were lyophilized, incubated in a desiccator with saturated sodium bromide 
solution for 14 days at room temperature to achieve a water activity (Aw) of 0.59 , and heated at $130^{\circ} \mathrm{C}$ for 10 minutes; 2 . Wet heating (W): heated in solution at $60{ }^{\circ} \mathrm{C}$ for 72 hours; 3 . Low-temperature dry heating (L): lyophilized and heated at $50{ }^{\circ} \mathrm{C}, 9$ hours at a relative humidity of $65 \%$ (equivalent to $\mathrm{Aw}$ 0.65) using a humidity control chamber (HCP108, Memmert, Schwabach, Germany). After heat processing, samples were dissolved in water to starting concentration (if in dry form) and centrifuged at $2900 \times \mathrm{g}$ for 30 minutes to remove insoluble particles. Supernatants were dialyzed at $4{ }^{\circ} \mathrm{C}$ against MQ-water using a membrane with a cut-off of 12-14 kDa (Thermo Fisher, Waltham, Massachusetts, USA), changing water twice every 2 hours before dialyzing overnight. Biological duplicates of each protein sample treatment were prepared and stored at $-20^{\circ} \mathrm{C}$. Samples were coded by treatment followed by the saccharide that was used for glycation. Saccharides were abbreviated to glu, gal, fru, lac, mal, md, FOS and GOS respectively. For example, "W-glu-BLG" represents the BLG sample mixed with glucose and heated in solution. The final protein concentration of each sample was determined by the Dumas method using Flash EA 1112 Nitrogen and Carbon Analyzer (Thermo Fischer, Waltham, Massachusetts, USA) with 6.29 as conversion factor.

\section{Fluorescent labelling}

Fluorescein isothiocyanate isomer I (FITC) was dissolved in DMSO to a concentration of $1 \mathrm{mg} / \mathrm{mL}$ and mixed with $1 \mathrm{mg} / \mathrm{mL}$ protein samples in borate buffer $(10 \mathrm{mM}, \mathrm{pH} 9)$ with a volume ratio 1:10. Mixtures were stored at $4{ }^{\circ} \mathrm{C}$ overnight and passed through a NAP-5 column (GE healthcare, Chicago, Illinois, USA) to remove unbound FITC. Protein samples were eluted from the column using sodium phosphate buffer. For cellular experiments, the protein samples were diluted in RPMI 1640 medium without phenol red (Gibco, Thermo Fisher, Waltham, Massachusetts, USA) to $0.1 \mathrm{mg} / \mathrm{mL}$. Fluorescence intensity in arbitrary units (a.u.) of each diluted sample was recorded by Infinite ${ }^{\circledR} 200$ PRO NanoQuant with i-control software (Tecan, Männedorf, Switzerland) using $495 \mathrm{~nm}$ and $525 \mathrm{~nm}$ as excitation and emission wavelength (Table 2.S2), to correct for differences in fluorescence intensity between samples.

\section{THP-1 macrophage cellular uptake}

The human monocytic leukemia cell line THP-1 (ATCC, Manassas, Virginia, USA) was differentiated into cells characteristic for resting macrophages (M0) as described by Chanput et al. [21] in Corning ${ }^{\circledR} 96$ Well TC-Treated Microplates (Life Sciences, Tewksbury, Massachusetts, USA) with $100 \mathrm{uL}$ cell suspension $\left(1 \times 10^{5}\right.$ cells $)$ in each well. After exposure to $100 \mu \mathrm{L}$ sample for 2 hours in a humidified incubator $\left(37^{\circ} \mathrm{C}, 5 \% \mathrm{CO}_{2}\right.$ ), cells were washed twice with PBS without $\mathrm{Ca}^{2+}$ and $\mathrm{Mg}^{2+}$ (Gibco, Thermo Fisher, Waltham, Massachusetts, USA) and incubated with $100 \mu \mathrm{L}$ of $0.25 \%$ Trypsin-EDTA (Gibco, Thermo Fisher, Waltham, Massachusetts, USA) for 5 minutes. Cell suspension in Trypsin-EDTA was 
centrifuged for 5 minutes at $450 \times \mathrm{g}$, then the cell pellet was re-suspended in $150 \mu \mathrm{L} 1 \%$ paraformaldehyde solution. The mean fluorescence intensity (MFI) of the FITC signal was recorded by flow cytometry (Accuri C6, BD Biosciences, Franklin Lakes, New Jersey, USA) of at least 5,000 cells for each sample using BD Accuri C6 software. For the calculation of relative protein uptake, the background fluorescence intensity of unstimulated cells was subtracted from the obtained MFI after sample exposure, was subsequently corrected for the sample FITC fluorescent signal measured by Tecan after labelling: $\left(\mathrm{MFI}_{\text {sample cell }}-\mathrm{MFI}_{\text {unstimulated cell }} /\right.$ Fluorecence ${ }_{\text {sample, }}$ and finally normalized to untreated BLG signals.

\section{Advance glycation end product (AGE) related fluorescence}

Protein samples were diluted to $1 \mathrm{mg} / \mathrm{mL}$ in sodium phosphate buffer and $100 \mu \mathrm{L}$ of each sample was added to a 96-well black polystyrene plate (Greiner CELLSTAR $^{\circledR}$, Kremsmünster, Austria). The autofluorescence (in a.u.) of AGEs was measured by Infinite ${ }^{\circledR} 200$ PRO NanoQuant with i-control software (Tecan, Männedorf, Switzerland) using $370 \mathrm{~nm}$ as excitation wavelength and $440 \mathrm{~nm}$ as emission wavelength. All samples were measured in duplicate and corrected against a sodium phosphate buffer control.

\section{o-Phtaldialdehyde (OPA) method}

The amount of free amino groups in untreated and treated BLG was determined by OPA based on Nielsen et al. [22]. Briefly, freshly prepared $200 \mu \mathrm{L}$ OPA reagent was added to $30 \mu \mathrm{L}$ of $1 \mathrm{mg} / \mathrm{mL}$ sample solution and incubated for 20 minutes at RT, after which the absorbance at $340 \mathrm{~nm}$ was measured with Infinite ${ }^{\circledR} 200$ PRO NanoQuant (Tecan, Männedorf, Switzerland). Sodium phosphate buffer was used as a control and each measurement was performed in duplicate. The amount of free amino groups was determined using an L-leucine standard curve (0.078-20 mM). The loss of amino groups was calculated as the percentage of blocked amino groups per protein relative to the free amino groups in untreated BLG.

\section{Amyloid structure detection}

The protein solution at $0.5 \mathrm{mg} / \mathrm{mL}$ was mixed with $50 \mu \mathrm{M}$ Thioflavin T (ThT) in sodium phosphate buffer using a volume ratio of 1:2. After 1 minute incubation at RT, $150 \mu \mathrm{L}$ of each sample mixture was measured in 96-well black polystyrene plate (Greiner CELLSTAR $^{\circledR}$, Kremsmünster, Austria) by Infinite ${ }^{\circledR}$ 200 PRO NanoQuant with i-control software (Tecan, Männedorf, Switzerland) in duplicate using 450 $\mathrm{nm}$ and $480 \mathrm{~nm}$ as excitation and emission wavelengths respectively. The fluorescence intensity (a.u.) 
of each sample was corrected for a sodium phosphate buffer control and divided by the value of untreated BLG.

\section{Secondary Structure prediction}

Using Circular Dichroism spectra (Figure 3.S4), percentages of secondary structure elements were estimated using BeStSel (http://bestsel.elte.hu/) which is specifically useful for detecting $\beta$-sheet-rich proteins like BLG [23].

\section{Classification of processed BLG in samples according to molecular weight}

Based on size exclusion chromatography (SEC) (Figure 3.S5), retention volume and area of peaks for each sample were analyzed using Unicorn 5.31 workstation software (GE healthcare, Chicago, Illinois, USA) while the molecular weight was calculated using a standard curve made by ovalbumin (43kDa), conalbumin $(75 \mathrm{kDa})$, aldolase $(158 \mathrm{kDa})$, catalase $(232 \mathrm{kDa})$, ferritin $(440 \mathrm{kDa})$ and blue dextran (2000kDa) from Gel Filtration Calibration Kits (GE healthcare, Chicago, Illinois, USA) Molecular weight of peaks were divided by the molecular weight of monomeric BLG (18.4 kDa) and values were categorized into monomer/dimer (1,2), oligomer (3-9), and polymer ( $\geq 10)$. Sample's molecular weight proportion of each category was calculated according to peak areas.

\section{Hydrophobicity measurement}

To determine the surface hydrophobicity of the samples using the 8-anilino-1-naphthalenesulfonic acid ammonium salt (ANS) analysis, the method of Alizadeh-Pasdar et al. [24] was modified. One mL of protein solution at $0.1 \mathrm{mg} / \mathrm{mL}$ in sodium phosphate buffer was mixed with $5 \mu \mathrm{L}$ ANS stock solution ( $8 \mathrm{mM}$ in sodium phosphate buffer, prepared in the dark). After incubation in the dark for $15 \mathrm{~min}, 100$ $\mu \mathrm{L}$ of each sample mixture was transferred to a 96 -well black polystyrene plate (Greiner CELLSTAR ${ }^{\circledR}$, Kremsmünster, Austria) and measured by Infinite ${ }^{\circledR} 200$ PRO NanoQuant with i-control software (Tecan, Männedorf, Switzerland) using $390 \mathrm{~nm}$ and $470 \mathrm{~nm}$ as excitation and emission wavelengths respectively. The fluorescence intensity (a.u.) of each sample was corrected for the sodium phosphate buffer control, divided by the value of untreated BLG, and shown as relative hydrophobicity (RH).

\section{Surface charge measurement}

The Zetasizer Nano ZS and Zetasizer software (Malvern Panalytica, Malvern, UK) were used to measure the zeta potential, which allows the inference of surface charge of molecules. Prior to measurement, samples were diluted in $M Q$ to $1 \mathrm{mg} / \mathrm{mL}$ and injected into a matched disposable capillary cell 
(DTS1070). The measurements were performed at $25^{\circ} \mathrm{C}$ and 40 volt and three sequential runs were averaged to obtain the results.

\section{Statistics}

All parameters are presented as means \pm standard deviation (SD) and differences between parameters were assessed for their significance with ANOVA using Prism 6 software. Differences with $p<0.05$ were considered significant. The Pearson correlation coefficient $(r)$ and two-tailed $p$ value were analyzed using Prism 6 software. Unscrambler software (CAMO, Oslo, Norway) was used to generate PCA plots integrating data from various measurements. For PCA, the dataset of variables was mean centered and weighted by $1 /$ standard deviation to limit the bias from the numerical value.

\section{Results}

\section{Heat treatment did not affect crucial parameters for cell cultures experimentation}

To link protein structural properties to immunogenicity, we treated BLG in the presence or absence of reducing saccharides with heating methods that variably induce glycation and aggregation. BLG was treated with the wet heating $(\mathrm{W})$ or dry heating at low-temperature $(\mathrm{L})$ or high-temperature $(\mathrm{H})$ methods in the presence of monosaccharides [glucose (glu), galactose (gal) or fructose (fru)], disaccharides [lactose (lac) or maltose (mal)], polysaccharides [maltodextrin (md), fructooligosaccharide (FOS) or galactooligosaccharide (GOS)] or in the absence of saccharides. We firstly analyzed solubility, $\mathrm{pH}$ and osmolality of the resulting BLG samples as these properties not only describe the physicochemical structure but are also critical cell culture parameters.

High-temperature dry heating of BLG reduced its solubility. When this treatment was performed in presence of monosaccharides the solubility was further and significantly reduced. In contrast, the addition of saccharides did not alter the solubility of low-temperature dry heated or wet heated BLG (Figure 3.S1). When dissolved in cell culture medium $(0.1 \mathrm{mg} / \mathrm{ml}$ protein), no significant differences were observed between medium and protein samples regarding $\mathrm{pH}$ and osmolality (Figure 3.S2).

\section{Wet heating most strongly increased BLG uptake by THP-1 macrophages}

We analyzed whether heat treatment of BLG affected the uptake of BLG by macrophages. To ensure that the observed effects were due to treatment-induced protein changes, we analyzed lipopolysaccharide (LPS) contamination in our samples as this may influence results in cellular immune assays. We observed a maximum of $0.155 \mathrm{ng}$ LPS contamination per $0.1 \mathrm{mg}$ of protein in all samples 
(Table 3.S1) which is well below the threshold at which LPS significantly influenced the uptake of fluorescent beads by THP-1 macrophages (i.e. $1000 \mathrm{ng} / \mathrm{ml}$; Figure 3.S3).

A

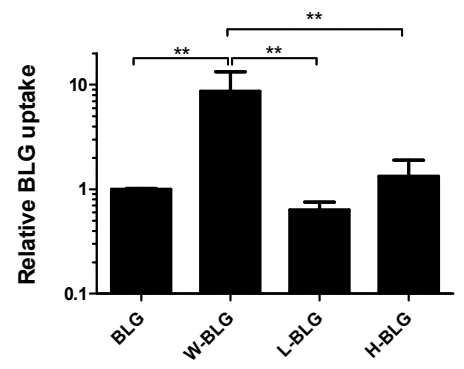

C

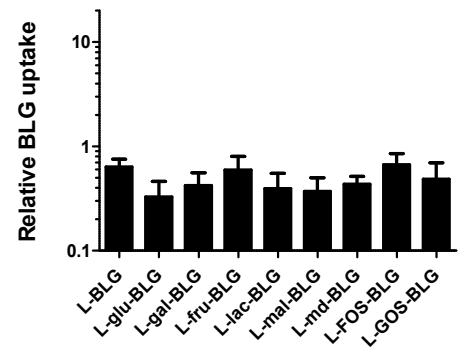

B

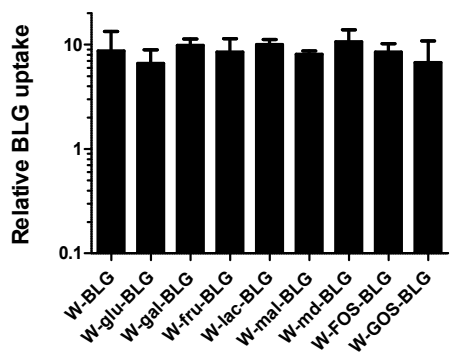

$\mathrm{D}$

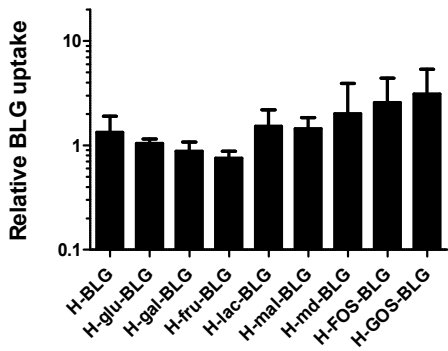

Figure 3.1 BLG phagocytosis by THP-1 macrophages is increased upon wet heating. BLG was untreated or heated (W, L or H) in absence or presence of saccharides (glu, gal, fru, lac, mal, md, FOS or GOS) and subsequently fluorescently labelled. BLG uptake by THP-1 macrophages was determined by flow cytometry as mean fluorescence intensity (MFI). BLG uptake was calculated for each sample and normalized for untreated BLG uptake. THP-1 macrophages were tested for uptake of untreated BLG or $B L G$ heat-treated in absence of sugars (A) and wet-heated (B), low-temperature dry-heated (C) and high-temperature dry-heated (D) BLG in presence of saccharides. Bars represent mean value $\pm S D$ of 4 independent experiments with 2 independent sample sets. Statistical differences were calculated with Tukey's Multiple Comparison Test (A) or Dunnett's Test (B-D): **p $<0.01$.

All heat-treated BLG samples were fluorescently labelled and their uptake by THP-1 macrophages was measured with flow cytometry and reported in mean fluorescence intensity (MFI) following 2 hours incubation. The heating method was found to exert a more pronounced effect on the uptake of BLG than the glycation with various saccharides. Wet-heated BLG, independent of the presence of saccharides, demonstrated significantly increased levels of uptake by the THP-1 macrophages (Figure 
3.1A and B). Within the dry-heated sample sets, a trend of lowered uptake of BLG was observed for samples exposed to low-temperature dry heating in the presence of saccharides and of increased uptake of BLG when treated with high-temperature dry heating in the presence of oligosaccharides (Figure 3.1C and D).

\section{Physicochemical characterization of BLG samples after heating}

Heat treatment, especially in presence of saccharides, led to loss of free amino groups and formation of AGE-related fluorescence

A

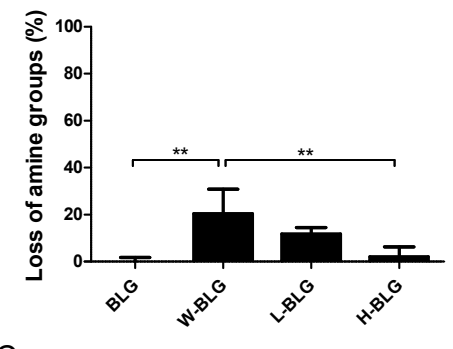

C

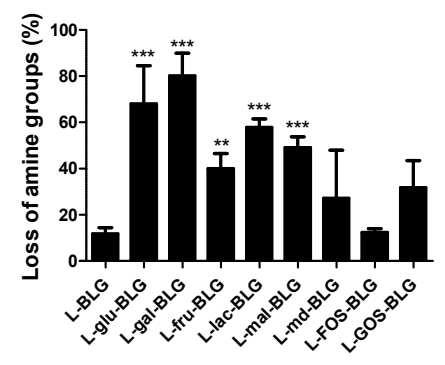

B

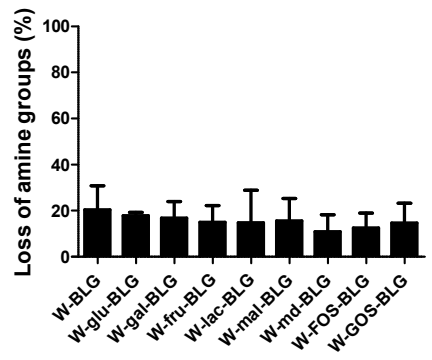

D

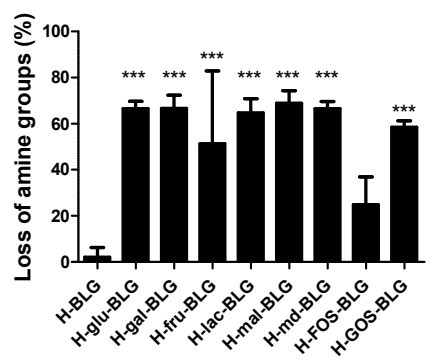

Figure 3.2 High-but also low-temperature dry heating in presence of saccharides of BLG led to a loss of free amine-groups. BLG was untreated or heated $(W, L$ or $H)$ in absence or presence of saccharides (glu, gal, fru, lac, mal, md, FOS or GOS). Glycation of untreated BLG or BLG heat-treated in absence of sugars $(A)$ and wet-heated $(B)$, low-temperature dry-heated $(C)$ and high-temperature dry-heated (D) $B L G$ in presence of saccharides was determined by loss of free amine-groups using OPA. Bars represent mean value $\pm S D$ of 4 independent experiments with 2 independent sample sets. Statistical differences were calculated with Tukey's Multiple Comparison Test (A) or Dunnett's Test (B-D): ${ }^{* *} p<0.01 ;{ }^{* * *} p<$ 0.001 . 
Heat treatment of protein in the presence of reducing saccharides is known to cause glycation which we measured using the OPA method to determine the loss of free amino groups. A significant loss of free amino groups, in comparison to untreated BLG, was detected when wet heating BLG, but not hightemperature or low-temperature dry heating in the absence of saccharides (Figure 3.2A). For wet heated samples, no further loss of free amino groups occurred upon incubation with any of the saccharides (Figure 3.2B). Contrastingly, when treating BLG with high- and low-temperature dry heating in the presence of any saccharides the loss of free amino groups was significantly increased, except md, FOS and GOS for low-temperature- and FOS for high-temperature dry heating (Figure 3.2C and D), with a higher loss of free amino groups compared to wet heated samples.

A

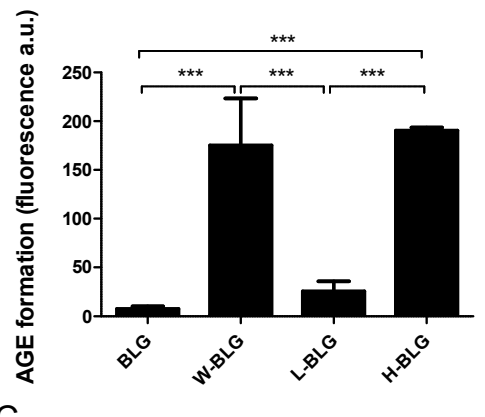

C

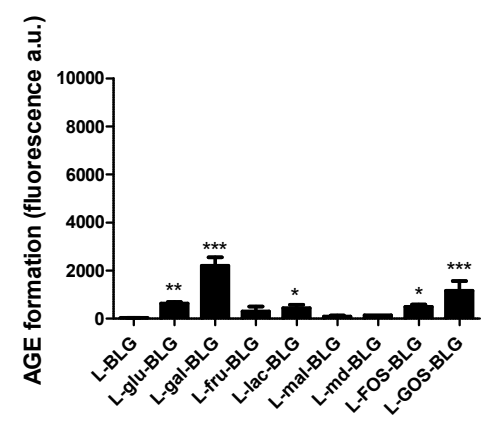

B
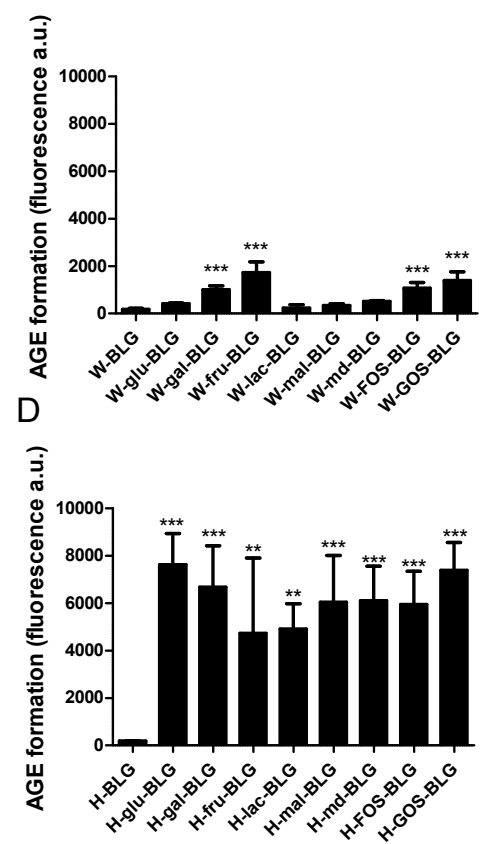

Figure 3.3 High-temperature dry heating in presence of saccharides increased BLG fluorescence which is correlated to advanced glycation end products. BLG was untreated or heated (W, $L$ or $H$ ) in absence or presence of saccharides (glu, gal, fru, lac, mal, md, FOS or GOS). Advanced glycation end-products in untreated BLG or BLG heat-treated in absence of sugars $(A)$ and wet-heated $(B)$, low-temperature dry-heated $(C)$ and high-temperature dry-heated $(D) B L G$ in presence of saccharides was determined by measuring fluorescence (370 nm exc./440 nm em.). Bars represent mean value \pm SD of 4 independent experiments with 2 independent sample sets. Statistical differences were calculated with Tukey's Multiple Comparison Test (A) or Dunnett's Test (B-D): * $p<0.05 ;{ }^{* *} p<0.01 ; * * p<0.001$. 
A next step in the chemical process is the formation of advanced glycation end products (AGEs)[25]. Fluorescence intensities at 370/440 nm (exc./em.) were used as an indicator for AGEs formation in the Maillard reaction [26-28]. In the absence of saccharides, wet-heating but also high-temperature dryheating of BLG induced a significant increase of the fluorescent signal compared to untreated BLG which suggested glycoxidation (Figure 3.3A). This, however, was negligible compared to the observed signals upon the addition of saccharides during heat-treatment. The addition of specific saccharides strongly and significantly increased the fluorescent signal of BLG following wet heating (i.e. gal, fru, FOS and GOS) or low-temperature dry heating (i.e. glu, gal, lac, FOS and GOS; Figure 3.3B and C). Addition of any saccharide in the high-temperature dry heating treatment of BLG significantly increased this signal to levels much higher than with either of the other two heating methods (Figure 3.3D).

A

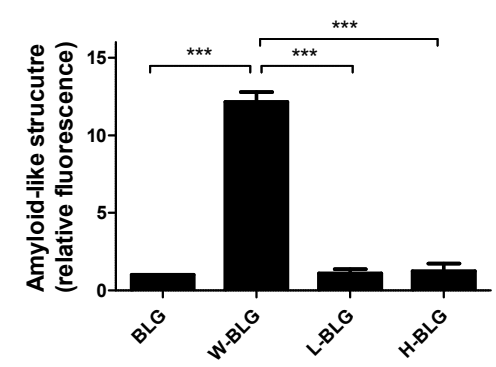

C

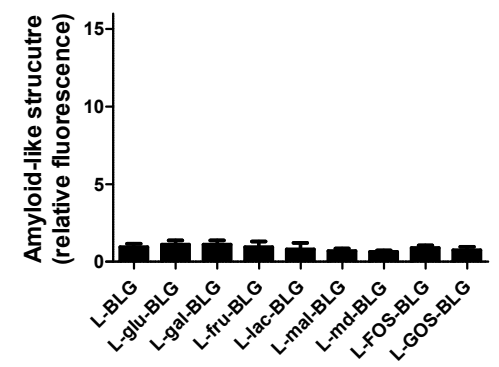

B

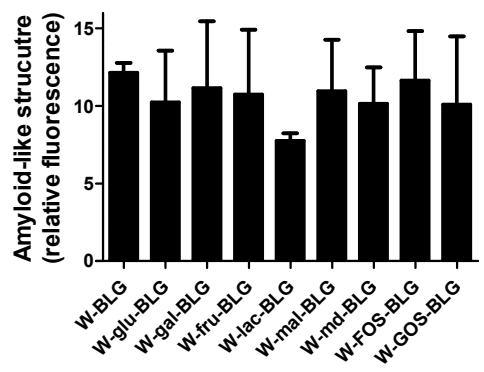

$\mathrm{D}$

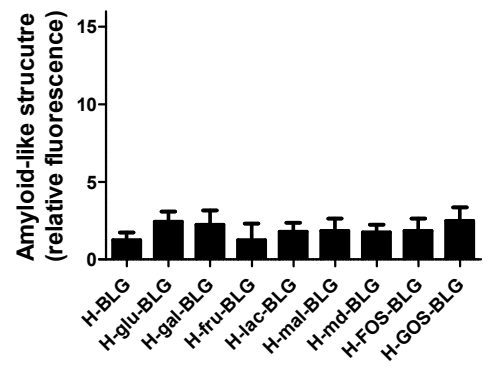

Figure 3.4 Wet heating of BLG increased the presence of amyloid-like structures. BLG was untreated or heated (W, L or $H$ ) in absence or presence of saccharides (glu, gal, fru, lac, mal, md, FOS or GOS). The presence of amyloid-like structures in untreated BLG or BLG heat-treated in absence of sugars $(A)$ and wet-heated $(B)$, low-temperature dry-heated $(C)$ and high-temperature dry-heated $(D) B L G$ in presence 
of saccharides was determined by ThT staining. Bars represent mean value $\pm S D$ of 4 independent experiments with 2 independent sample sets. Statistical differences were calculated with Tukey's Multiple Comparison Test (A) or Dunnett's Test (B-D): ***p<0.001.

\section{Wet heating reduced 6-sheet formation in BLG but increases amyloid-like structures and $\alpha$-helices}

We analyzed changes in protein structures by determining amyloid-like structure formation (Figure 3.4) and presence of $\alpha$-helix and $\beta$-sheets (Figure 3.5). Amyloid-like structure formation was measured with ThT staining which revealed a significant increase compared to untreated BLG only upon wet heating (Figure 3.4A). The addition of saccharides during the heat treatments did not significantly affect the formation of amyloid structures compared to treatment of untreated BLG (Figure 3.4B-C). The prediction of $\alpha$-helix and $\beta$-sheet proportion of the BLG samples was calculated with a software package using the far UV protein spectrum acquired with CD (Figure 3.S4). The spectra of BLG samples treated with low-temperature dry heating were similar to untreated BLG, whereas wet heating of BLG alters the spectra in a saccharide-independent manner. High-temperature dry heating of BLG altered the spectrum compared to untreated BLG and this change was exacerbated upon the addition of saccharides.

Quantification of $\alpha$-helix and $\beta$-sheet revealed some significant differences (Figure 3.5). Wet heating significantly increased the percentages of $\alpha$-helices and reduced the percentages of $\beta$-sheets in BLG (Figure 3.5A, B, E and F). Saccharide addition during treatment did not affect the percentages of $\alpha$ helix or $\beta$-sheet in the BLG samples, except for glucose which increased $\alpha$-helix and lowered $\beta$-sheet percentages upon high-temperature dry heating and maltodextrose which lowered $\alpha$-helix percentages upon low-temperature dry heating (Figure 3.5C, D, G and H).

\section{Wet heating, but also high-temperature dry heating in presence of saccharides, affected the formation of soluble aggregates in BLG samples}

Molecular weights were categorized into monomeric or dimeric BLG, oligomeric (3-9 BLG molecules) and polymeric (> 10 BLG molecules) (Figure 3.6). Low-temperature dry heated BLG samples consisted mainly of dimers which was similar to untreated BLG, whereas wet heated BLG samples mainly consisted of polymers. For both treatments the addition of saccharides did not affect the aggregation of BLG. Besides insoluble aggregates, high-temperature dry heating induced a relatively big amount of soluble oligomers compared to untreated BLG, which appeared to increase with the addition of saccharides in the order of monosaccharide < polysaccharides < oligosaccharide. High-temperature dry heating also appeared to induce the dissociation of the normal dimeric form of BLG in solution (Figure 3.S5). 
A

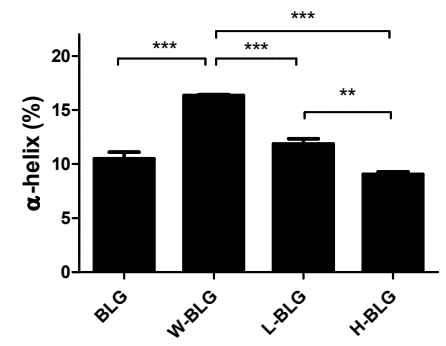

C

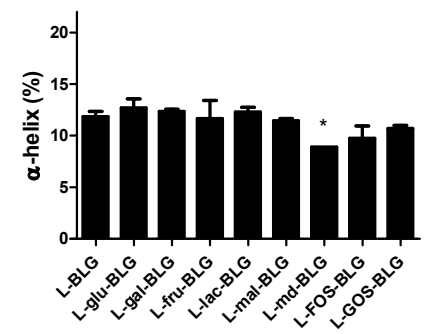

E

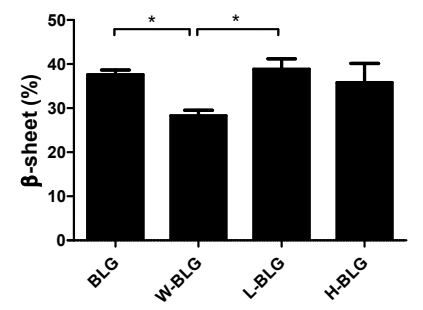

G

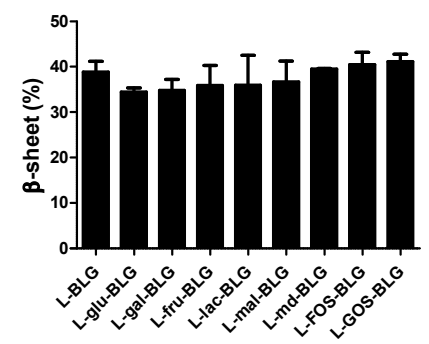

B

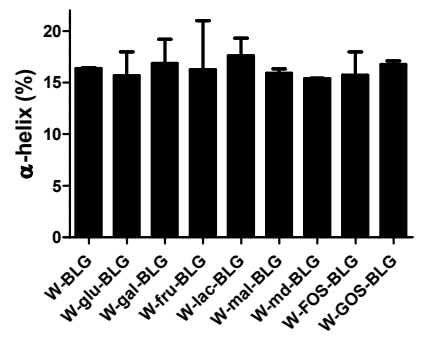

D

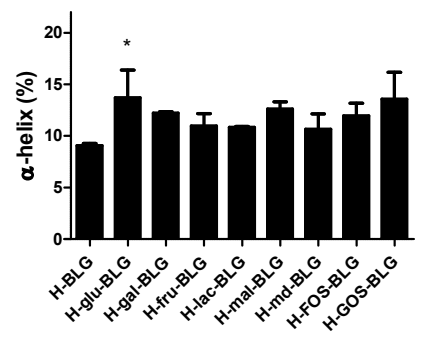

$\mathrm{F}$

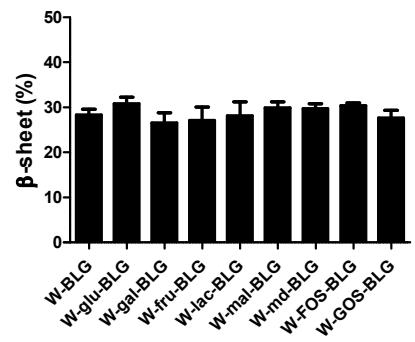

$\mathrm{H}$

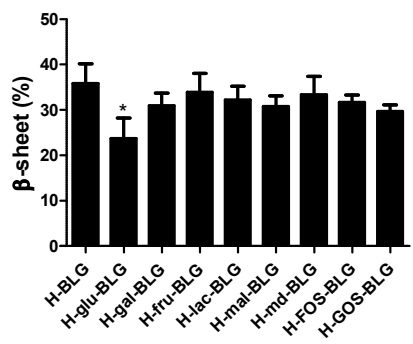

Figure 3.5 Wet heating increased $\alpha$-helix-and lowered B-sheet proportions in BLG. BLG was untreated or heated (W, Lor H) in absence or presence of saccharides (glu, gal, fru, lac, mal, md, FOS or GOS). The percentages of $\alpha$-helix $(A-D)$ and $B$-sheet $(E-H)$ structures in untreated BLG or BLG heat-treated in 
absence of sugars $(A, E)$ and wet-heated $(B, F)$, low-temperature dry-heated $(C, G)$ and hightemperature dry-heated $(D, H) B L G$ in presence of saccharides were determined using BeStSel software to analyse UV-CD spectra. Bars represent mean value $\pm S D$ of 2 independent sample sets, each averaging 10 repeated measurements. Statistical differences were calculated with Tukey's Multiple Comparison Test (A, E) or Dunnett's Test (B-D, F-H): *p<0.05; ${ }^{* *} p<0.01 ; * * p<0.001$.

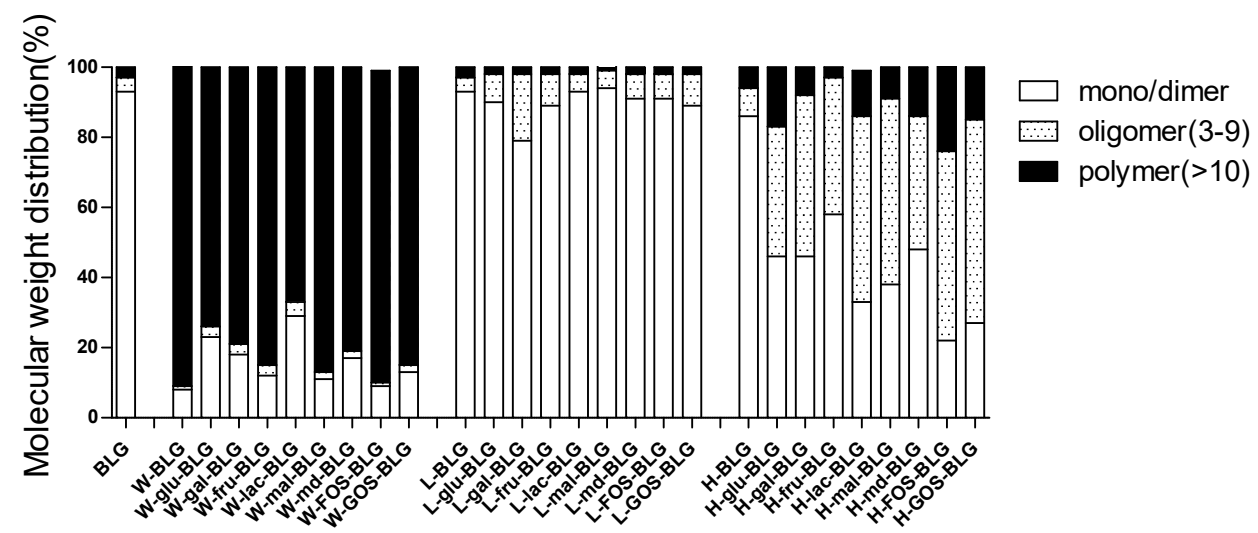

Figure 3.6 An increase in BLG multimers was observed upon high-temperature dry heating in presence of sugars but particularly upon wet heating. BLG was untreated or heated (W, L or $H)$ in absence or presence of saccharides (glu, gal, fru, lac, mal, $\mathrm{md}$, FOS or GOS) and measured for aggregation using SEC chromatography. Stacked bars represent averages following single measurements of 2 independent sample sets.

Heat treatments but also the presence of saccharides affected the zeta potential and exposure of hydrophobic regions of $B L G$

The changes in tertiary and quaternary structure might also result in exposure of hydrophobic regions which could trigger immune responses [29]. Hydrophobicity was determined with the ANS measurement which revealed a significant increase upon wet heating of untreated BLG (Figure 3.7A). The addition of saccharides did not alter the exposure of hydrophobic regions of the BLG protein for any of the three treatments (Figure 3.7B-D).

Finally, we determined the zeta potential as protein surface property. Wet heating of BLG significantly decreased the zeta potential compared to untreated BLG (Figure 3.8A). This effect, however, was counteracted by the addition of any of the saccharides during heating, which increased the zeta potential, except glucose and GOS (Figure 3.8B). In contrast to wet heating, high-temperature dry 
heating showed a trend towards an increased zeta potential of untreated BLG, which further and significantly increased upon the addition glucose, galactose, fructose, lactose and FOS (Figure 3.8D). Finally, low-temperature dry heating in absence or presence of saccharides did not alter the zeta potential compared to untreated BLG (Figure 3.8C).

A

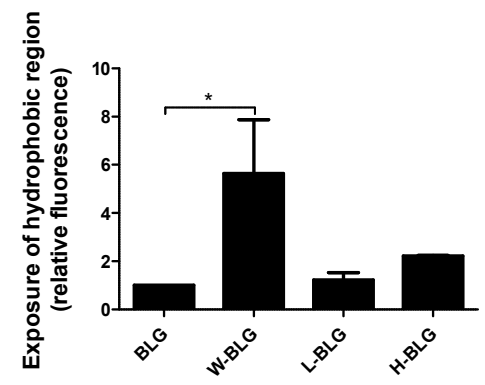

C

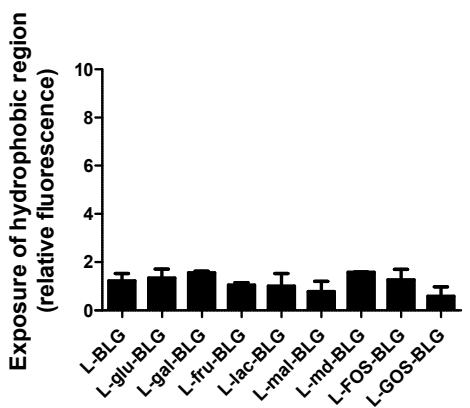

B

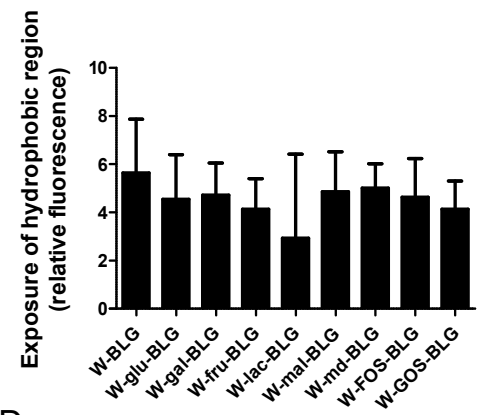

D

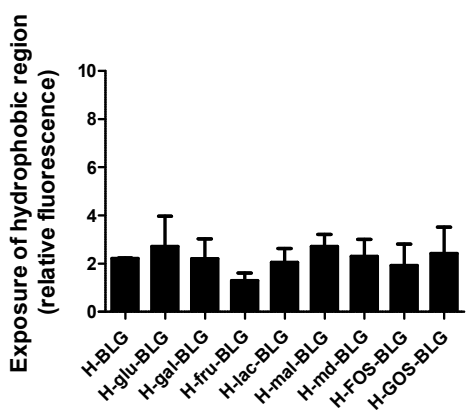

Figure 3.7 Wet heating increased exposure of hydrophobic regions of BLG. BLG was untreated or heated $(W, L$ or $H)$ in absence or presence of saccharides (glu, gal, fru, lac, mal, $m d$, FOS or GOS). The exposure of hydrophobic regions in untreated BLG or BLG heat-treated in absence of sugars ( $A$ ) and wet-heated (B), low-temperature dry-heated (C) and high-temperature dry-heated (D) BLG in presence of saccharides was determined by applying ANS fluorescence normalized to untreated BLG. Bars represent mean value $\pm S D$ of 4 independent experiments with 2 independent sample sets. Statistical differences were calculated with Tukey's Multiple Comparison Test (A) or Dunnett's Test (B-D): * $p<0.05$. 
A

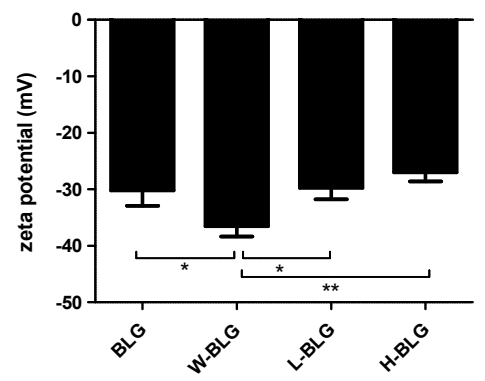

C

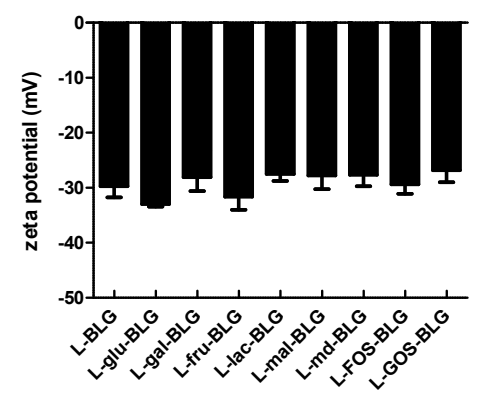

B

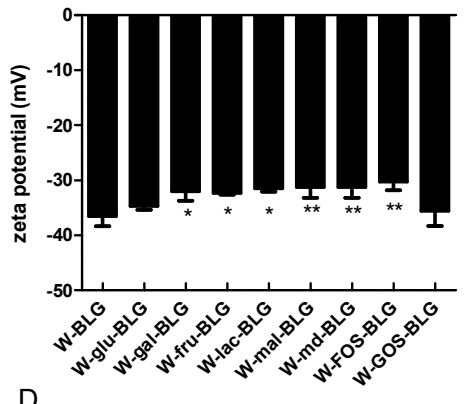

$\mathrm{D}$

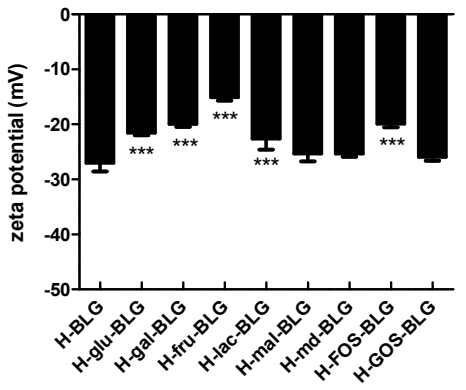

Figure 3.8 Wet heating alone, wet heating or high-temperature dry heating in the presence of saccharides affected the zeta potential of BLG. BLG was untreated or heated (W, L or $H)$ in absence or presence of saccharides ( $g l u, g a l$, fru, lac, mal, $m d$, FOS or GOS). The zeta potential in untreated BLG or $B L G$ heat-treated in absence of sugars $(A)$ and wet-heated $(B)$, low-temperature dry-heated $(C)$ and high-temperature dry-heated (D) BLG in presence of saccharides was measured using the Zetasizer. Bars represent mean value $\pm S D$ of 2 independent sample sets, each averaging 3 repeat measurements. Statistical differences were calculated with Tukey's Multiple Comparison Test (A) or Dunnett's Test (B$D):{ }^{*} p<0.05 ;{ }^{* *} p<0.01 ;{ }^{* *} p<0.001$.

\section{Correlation analysis \& principle component analysis (PCA)}

Heat treatments as well as addition of saccharides during the processing have shown to variably affect the physicochemical characteristics of BLG. We performed a correlation analysis which included all samples and measurements. The correlation analysis (Figure 3.9) demonstrated that the transition from monomer/dimer to polymers, percentage of $\alpha$-helix and amyloid-like structures, and hydrophobicity correlated strongly and significantly with uptake of BLG by THP-1 macrophages $(|r|>$ $0.8, p<0.0001)$. There was a moderate inverse correlation between uptake and percentage of $\beta$-sheet structures, zeta potential, and loss of amino groups (Figure 3.56). We also performed a principle 
component analysis (PCA) (Figure 3.10A) which demonstrated that the type of processing, according to PC1 and PC2 was strongly related to the uptake of BLG. Wet heated samples were taken up most efficiently (Figure 3.1B), whereas the type of saccharide did not influence the clustering of wet heated BLG samples in the PCA. In the PCA, untreated BLG clustered together with BLG that was treated with low-temperature dry heating in absence or presence of the saccharides and with high-temperature dry heated BLG in absence of saccharides, indicating that these samples were modified the least. The lowand high-temperature dry heated BLG in the presence of saccharides, however, clustered separately.

The type of sugar only influenced clustering for the high-temperature dry heated samples. Detailed analysis including only high-temperature dry heated BLG and untreated BLG (Figure 3.10B) confirmed that high-temperature dry heated BLG in presence of either monomeric, dimeric and oligomeric saccharides resulted in separate clusters based on their physicochemical characteristics. Uptake seemed higher for BLG that was glycated with longer saccharides (Figure 3.1D), but also in this case the uptake correlated with the occurrence of larger soluble aggregates (Figure 3.6).

A

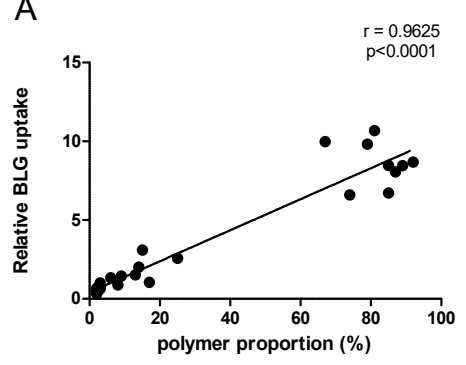

C

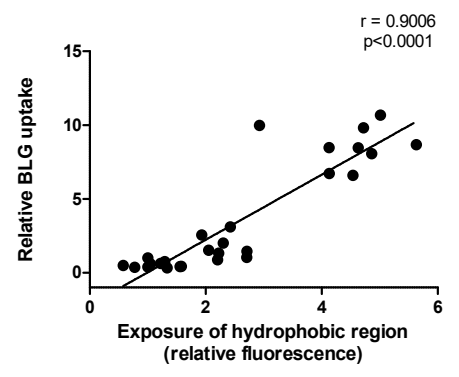

B

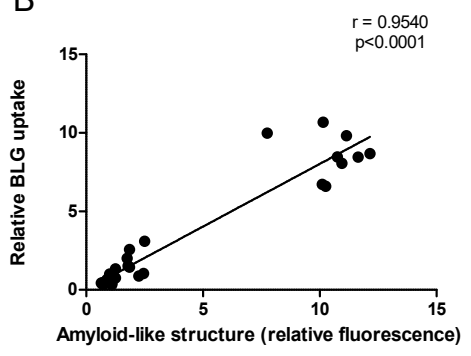

$\mathrm{D}$

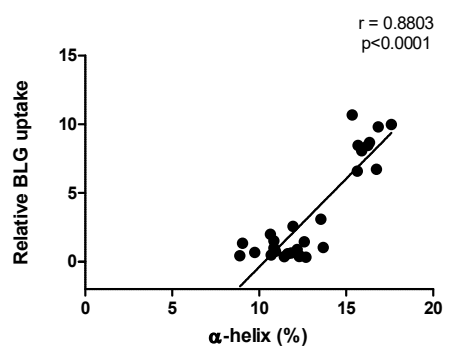

Figure 3.9 Uptake of BLG by THP-1 macrophage correlates with aggregation, presence of amyloid-like structure, hydrophobicity, and percentage of $\alpha$-helix. Uptake of thermally treated BLG in the presence or absence of saccharides was plotted against polymer proportion, amyloid-like structure, exposure of hydrophobic region and $\alpha$-helix. The correlations were strong with $|r|>0.8$ and significant with $p<$ 0.0001 . 
A

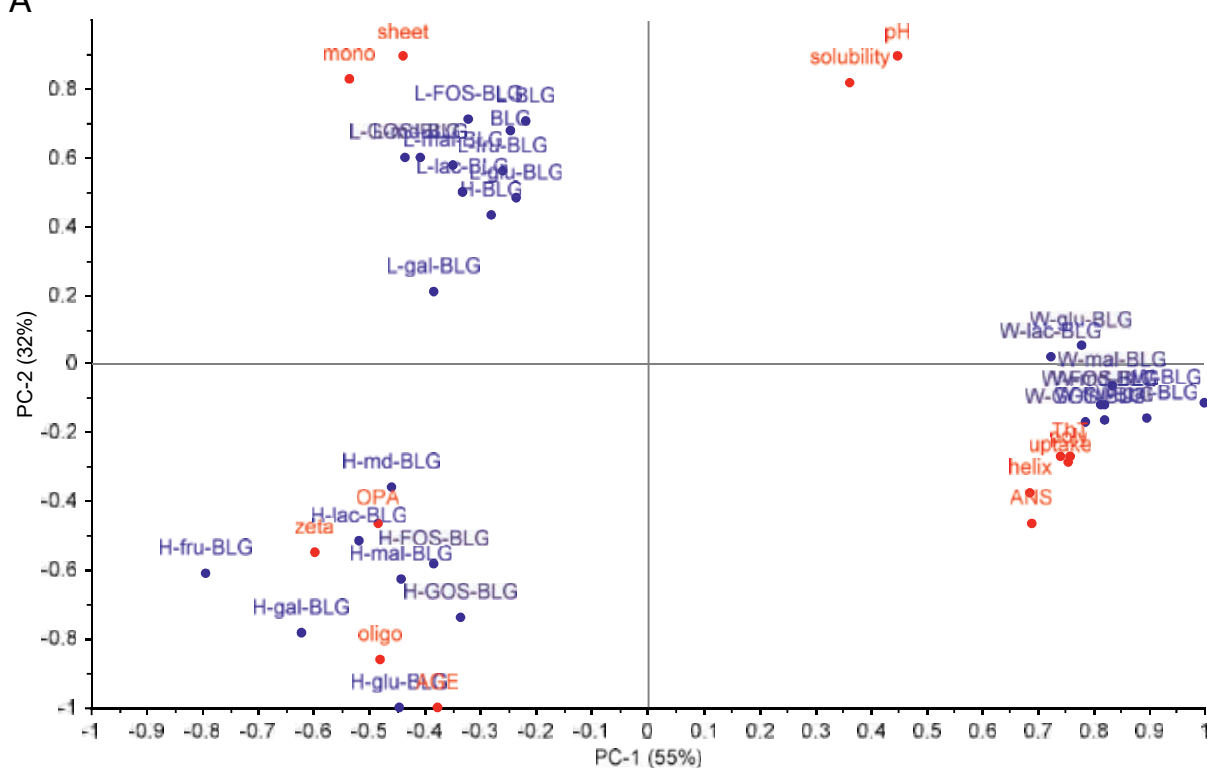

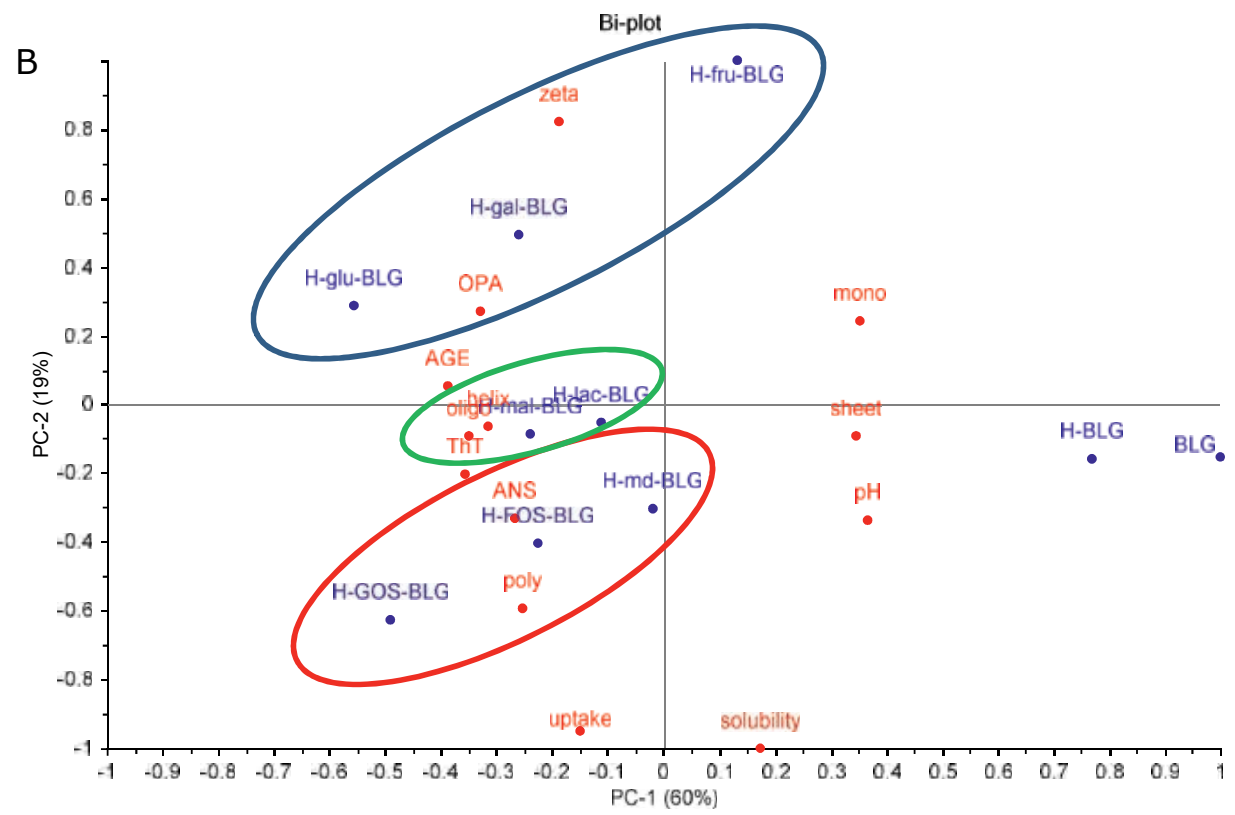

Figure 3.10 Uptake of BLG correlated with wet heating and BLG exposure of hydrophobic regions, percentage of $\alpha$-helix, amyloid-like structures and polymers. BLG was untreated or heated (W, L or $H)$ in absence or presence of saccharides (glu, gal, fru, lac, mal, md, FOS or GOS) and tested for solubility, pH, uptake by THP-1 macrophages (uptake), AGE formation (AGE), glycation (OPA), amyloid-like 
structure formation (ThT), percentage of $\alpha$-helix (helix) or b-sheet (sheet), aggregation [monomer and dimer (mono), oligomers (oligo), polymers (poly)], exposure of hydrophobic regions (ANS) and surface charge (zeta). All parameters (A) or all high-temperature dry heating-related parameters $(B)$ were included in a principle component analysis bi-plot using the Unscrambler software and scattered according to heat treatment and saccharides, respectively. In Figure $B$ the clustering of monosaccharides (blue circle), disaccharides (green circle) and oligosaccharides (red circle) is indicated.

\section{Discussion}

Processing of food affects the immunogenicity of proteins contained therein, as shown for the increased allergenic potential of peanuts upon dry roasting [30] and of BLG upon crosslinking [31]. The suggestion is imminent that structural changes, resulting from the processing of such proteins, form the molecular basis of this increased immuno- or allergenicity. Structural damage to proteins in relation to their immunogenicity are already apparent from the DAMP-concept as postulated by Matzinger [32] or from the precautions that are taken to reduce shear-related damage and aggregate formation in the preparation of proteins for pharmaceutical applications [33]. In this paper, we described how wet heating and low- or high-temperature dry heating affects the uptake of $\beta$ lactoglobulin by THP-1-derived macrophages. BLG was chosen as model because it is a known allergen that is relevant in the context of cow milk allergy. In addition, it is not digested in the human gastric compartment and stable for at least one hour in the jejunal compartment [34] and therefore relatively undigested protein fragments can interact with intestinal immune cells.

We expected the physicochemical characteristics of glycation and AGE formation to most strongly impact protein uptake by macrophages as these properties have been demonstrated to affect protein immunogenicity [35-39]. Low- and high-temperature dry heating of BLG in presence of saccharides, as also previously observed $[5,18]$, resulted in loss of free amino groups suggestive of glycation (Figure 3.2). High-temperature dry heating also induced the formation of AGE-related fluorescence (Figure 3.3) although AGEs were not directly measured quantitatively. Both heating methods, however, did not significantly increase BLG uptake by THP-1 macrophages (Figure 3.1). This contrasts with previous studies which demonstrated that glycation of OVA drives the uptake by dendritic cells $[3,4,40]$. Wet heating also significantly increased the fluorescent signal linked to AGE formation and demonstrated a loss of free amino groups when compared to untreated BLG. Both signals were, however, lower compared to low- or high-temperature dry heated BLG in presence of sugars and could result from oxidation or other secondary structural changes $[41,42]$. Surprisingly, analysis towards receptors involved in the uptake of glycated OVA (AGE-OVA) revealed that not the documented receptors for 
glycated proteins (i.e. RAGE, galectin-3 and CD36 [39]) but the scavenger receptor class A I/II, recognized as DAMP-receptor, was important for the uptake by DCs. Further comparison showed that, in these studies, AGE-OVA was obtained by heating at $50^{\circ} \mathrm{C}$ for 6 weeks in solution [43] in which the lengthy incubation period will cause more extensive glycation. As a result, this method holds the middle between our wet heating and dry heating method. Taken together, it appears possible that the increased uptake of AGE-OVA should not be attributed solely to the glycation and AGE formation (i.e. typical physicochemical characteristics of dry heated proteins) and that other wet heating-induced physicochemical changes played a role as well.

We found that the process of wet heating of BLG, independent of saccharide presence, induced a significant increase in protein uptake by THP-1 macrophages. Using a correlation and PCA analysis, we demonstrated that the uptake of BLG following wet heating positively was correlated with physicochemical parameters of polymerization, exposure of hydrophobic regions, presence of amyloid-like structures and $\alpha$-helices and, moderate, correlated inversely with $\beta$-sheets, surface charge and loss of amino groups (Figure 3.9 and Figure 3.56). Most of these physicochemical protein characteristics have individually been linked to immune responses and/or increased phagocytosis by macrophages. Seong and Matzinger concluded that almost all immuno-stimulatory molecules were either entirely hydrophobic or contained exposed hydrophobic regions which can trigger various receptors such as CD14, toll-like receptors (TLRs) or scavenger receptors that recognize dangerassociated molecular patterns (DAMPs) [32]. Furthermore, they also point out that hydrophobic structures are prone to aggregation into (insoluble) particles. Aggregation itself has also been linked to adverse immune responses through stimulating TLRs via intrinsic factors such as size, concentration and generation of neo-epitopes [44, 45]. Of note, these immune responses appear to result from stimulation with soluble aggregates and not insoluble large aggregates [46] as shown with fractions of aggregated amyloid beta. Amyloid beta may be the best-known example of aggregated protein interacting with the immune system. It is cleared in the brain by macrophages and microglia via binding by scavenger receptors and TLRs $[47,48]$, which forms a rationale for the observed relation between amyloid-like structures in BLG and uptake by THP-1 macrophages. From literature, the relation between uptake and the increased presence of $\alpha$-helix structures is less apparent. Similar to wet heating, high-temperature dry heating in the presence of glucose increased the proportion of $\alpha$-helices in BLG (Figure 3.5D). However, this sample didn't show increased protein uptake which dismisses the importance of $\alpha$-helix structures for uptake of BLG by THP-1 macrophages.

Correlation of exposure of hydrophobic regions, protein polymerization (> 10 monomers) and presence of amyloid-like structures to wet heating-mediated enhanced uptake of BLG was also most apparent from the individual figures. Of interest, these parameters are all linked to receptor mediated 
uptake in contrast to $\alpha$-helix and surface charge. The fluorescent dye ThT has been used for decades to detect amyloid structures for a variety of applications. More recently, the molecular base for this interaction has been studied. This revealed that in addition to detecting amyloid fibrils, a loss of protein structure and exposure of hydrophobic regions can also lead to ThT binding in hydrophobic pockets of globular proteins, which putatively requires 5 aromatic and/or hydrophobic residues [49, 50]. The observed ThT signal for wet heated BLG samples might therefore result from exposed hydrophobic regions and provide a false-positive ThT signal. As already discussed above and shown in relation to ANS measurements [51], aggregation can be a result of exposure of hydrophobic regions. We therefore postulate that the exposure of hydrophobic regions is the leading physicochemical characteristic for the observed uptake by THP-1 macrophages of wet heated BLG samples. This is supported by multiple studies, using various types of particles, that correlated a hydrophobic particle surface to increased and more rapid uptake by immune cells [52-55].

\section{Conclusion}

We have demonstrated that wet heating of BLG increased the exposure of hydrophobic regions and led to the formation of soluble polymeric aggregates, which we hypothesize are at the basis of the observed increased uptake by macrophages. As indicated above, thermally processed proteins have more widely been demonstrated to be able to activate the immune system. The physiological consequences of such a trigger to the immune system remains to be established but may be relevant to explain the increased incidences of inflammation-associated health effects. 


\section{References}

1. Puig, C., et al., Effect of processing on the composition of infant formulas. MilchwissenschaftMilk Science International, 2003. 58(9-10): p. 476-480.

2. Zhang, Q.B., et al., A Perspective on the Maillard Reaction and the Analysis of Protein Glycation by Mass Spectrometry: Probing the Pathogenesis of Chronic Disease. Journal of Proteome Research, 2009. 8(2): p. 754-769.

3. Hilmenyuk, T., et al., Effects of glycation of the model food allergen ovalbumin on antigen uptake and presentation by human dendritic cells. Immunology, 2010. 129(3): p. 437-445.

4. Ilchmann, A., et al., Glycation of a food allergen by the Maillard reaction enhances its T-cell immunogenicity: Role of macrophage scavenger receptor class A type I and II. Journal of Allergy and Clinical Immunology, 2010. 125(1): p. 175-183.

5. Liu, F.H., et al., The decrease in the IgG-binding capacity of intensively dry heated whey proteins is associated with intense Maillard reaction, structural changes of the proteins and formation of RAGE-ligands. Food \& Function, 2016. 7(1): p. 239-249.

6. Rytkonen, J., et al., Effect of heat denaturation on beta-lactoglobulin-induced gastrointestinal sensitization in rats: Denatured beta $L G$ induces a more intensive local immunologic response than native beta LG. Pediatric Allergy and Immunology, 2002. 13(4): p. 269-277.

7. $\mathrm{Bu}, \mathrm{G} . \mathrm{H}$. , et al., Effect of heat treatment on the antigenicity of bovine -lactalbumin and lactoglobulin in whey protein isolate. Food and Agricultural Immunology, 2009. 20(3): p. 195206.

8. Bleumink, E. and L. Berrens, Synthetic Approaches to Biological Activity of Beta-Lactoglobulin in Human Allergy to Cows Milk. Nature, 1966. 212(5061): p. 541-\&.

9. Alexander, L.J., et al., Complete Sequence of the Bovine Beta-Lactoglobulin Cdna. Nucleic Acids Research, 1989. 17(16): p. 6739-6739.

10. Mousavi, S.H.A., A.K. Bordbar, and T. Haertle, Changes in structure and in interactions of heattreated bovine beta-lactoglobulin. Protein and Peptide Letters, 2008. 15(8): p. 818-825.

11. Palazolo, G., et al., Heat treatment of beta-lactoglobulin: Structural changes studied by partitioning and fluorescence. Journal of Agricultural and Food Chemistry, 2000. 48(9): p. 38173822.

12. Chobert, J.M., et al., Impact of Maillard type glycation on properties of beta-lactoglobulin. Biotechnology Advances, 2006. 24(6): p. 629-632.

13. Chevalier, F., et al., Maillard glycation of beta-lactoglobulin induces conformation changes. Nahrung-Food, 2002. 46(2): p. 58-63.

14. Chevalier, F., et al., Maillard glycation of beta-lactoglobulin with several sugars: comparative study of the properties of the obtained polymers and of the substituted sites. Lait, 2001. 81(5): p. 651-666.

15. Uribarri, J., et al., Advanced Glycation End Products in Foods and a Practical Guide to Their Reduction in the Diet. Journal of the American Dietetic Association, 2010. 110(6): p. 911-916.

16. Chun, S.H., et al., Effects of Glycated Whey Protein Concentrate on Pro-inflammatory Cytokine Expression and Phagocytic Activity in RAW264.7 Macrophages. Biological \& Pharmaceutical Bulletin, 2016. 39(2): p. 199-206.

17. Chevalier, F., et al., Improvement of functional properties of beta-lactoglobulin glycated through the Maillard reaction is related to the nature of the sugar. International Dairy Journal, 2001. 11(3): p. 145-152.

18. Deng, Y., et al., Effect of Maillard induced glycation on protein hydrolysis by lysine/arginine and non-lysine/arginine specific proteases. Food Hydrocolloids, 2017. 69: p. 210-219.

19. de Jongh, H.H.J., T. Groneveld, and J. de Groot, Mild isolation procedure discloses new protein structural properties of beta-lactoglobulin. Journal of Dairy Science, 2001. 84(3): p. 562-571.

20. Liu, F.H., et al., Generation of Soluble Advanced Glycation End Products Receptor (sRAGE)Binding Ligands during Extensive Heat Treatment of Whey Protein/Lactose Mixtures Is 
Dependent on Glycation and Aggregation. Journal of Agricultural and Food Chemistry, 2016. 64(33): p. 6477-6486.

21. Chanput, W., et al., Characterization of polarized THP-1 macrophages and polarizing ability of LPS and food compounds. Food \& Function, 2013. 4(2): p. 266-276.

22. Nielsen, P.M., D. Petersen, and C. Dambmann, Improved method for determining food protein degree of hydrolysis. Journal of Food Science, 2001. 66(5): p. 642-646.

23. Micsonai, A., et al., Accurate secondary structure prediction and fold recognition for circular dichroism spectroscopy. Proceedings of the National Academy of Sciences of the United States of America, 2015. 112(24): p. E3095-E3103.

24. Alizadeh-Pasdar, N. and E.C.Y. Li-Chan, Application of PRODAN fluorescent probe to measure surface hydrophobicity of proteins interacting with kappa-carrageenan. Food Hydrocolloids, 2001. 15(3): p. 285-294.

25. Goldberg, T., et al., Advanced glycoxidation end products in commonly consumed foods. Journal of the American Dietetic Association, 2004. 104(8): p. 1287-1291.

26. Nakamura, K., Y. Nakazawa, and K. lenaga, Acid-stable fluorescent advanced glycation end products: Vesperlysines $A, B$, and $C$ are formed as crosslinked products in the maillard reaction between lysine or proteins with glucose. Biochemical and Biophysical Research Communications, 1997. 232(1): p. 227-230.

27. Yamaguchi, K., et al., Determination of Furosine and Fluorescence as Markers of the Maillard Reaction for the Evaluation of Meat Products during Actual Cooking Conditions. Food Science and Technology Research, 2012. 18(1): p. 67-76.

28. Sero, L., et al., Tuning a 96-Well Microtiter Plate Fluorescence-Based Assay to Identify AGE Inhibitors in Crude Plant Extracts. Molecules, 2013. 18(11): p. 14320-14339.

29. Moyano, D.F., et al., Nanoparticle Hydrophobicity Dictates Immune Response. Journal of the American Chemical Society, 2012. 134(9): p. 3965-3967.

30. Moghaddam, A.E., et al., Dry roasting enhances peanut-induced allergic sensitization across mucosal and cutaneous routes in mice. Journal of Allergy and Clinical Immunology, 2014. 134(6): p. 1453-1456.

31. Stojadinovic, M., et al., Cross-Linking of beta-Lactoglobulin Enhances Allergic Sensitization Through Changes in Cellular Uptake and Processing. Toxicological Sciences, 2014. 140(1): p. 224-235.

32. Seong, S.Y. and P. Matzinger, Hydrophobicity: an ancient damage-associated molecular pattern that initiates innate immune responses. Nature Reviews Immunology, 2004. 4(6): p. 469-478.

33. Bee, J.S., T.J. Goletz, and J.A. Ragheb, The future of protein particle characterization and understanding its potential to diminish the immunogenicity of biopharmaceuticals: a shared perspective. J Pharm Sci, 2012. 101(10): p. 3580-5.

34. Sanchon, J., et al., Protein degradation and peptide release from milk proteins in human jejunum. Comparison with in vitro gastrointestinal simulation. Food Chemistry, 2018. 239: $\mathrm{p}$. 486-494.

35. Price, C.L., et al., Advanced glycation end products modulate the maturation and function of peripheral blood dendritic cells. Diabetes, 2004. 53(6): p. 1452-1458.

36. Buttari, B., et al., Advanced glycation end products of human beta(2) glycoprotein I modulate the maturation and function of DCs. Blood, 2011. 117(23): p. 6152-6161.

37. Haucke, E., et al., Glycation of extracellular matrix proteins impairs migration of immune cells. Wound Repair Regen, 2014. 22(2): p. 239-45.

38. Nedic, O., et al., Molecular effects of advanced glycation end products on cell signalling pathways, ageing and pathophysiology. Free Radical Research, 2013. 47: p. 28-38.

39. Ott, C., et al., Role of advanced glycation end products in cellular signaling. Redox Biology, 2014. 2: p. 411-429.

40. Heilmann, M., et al., Ovalbumin Modified with Pyrraline, a Maillard Reaction Product, shows Enhanced T-cell Immunogenicity. Journal of Biological Chemistry, 2014. 289(11): p. 7919-7928. 
41. Beisswenger, P.J., et al., Two Fluorescent Wavelengths, 440(ex)/520(em) nm and $370(\mathrm{ex}) / 440(\mathrm{em}) \mathrm{nm}$, Reflect Advanced Glycation and Oxidation End Products in Human Skin Without Diabetes. Diabetes Technology \& Therapeutics, 2012. 14(3): p. 285-292.

42. Soladoye, O.P., et al., Protein Oxidation in Processed Meat: Mechanisms and Potential Implications on Human Health. Comprehensive Reviews in Food Science and Food Safety, 2015. 14(2): p. 106-122.

43. Gasic-Milenkovic, J., et al., beta-amyloid peptide potentiates inflammatory responses induced by lipopolysaccharide, interferon-gamma and 'advanced glycation endproducts' in a murine microglia cell line. European Journal of Neuroscience, 2003. 17(4): p. 813-821.

44. Moussa, E.M., et al., Immunogenicity of Therapeutic Protein Aggregates. Journal of Pharmaceutical Sciences, 2016. 105(2): p. 417-430.

45. Joubert, M.K., et al., Highly Aggregated Antibody Therapeutics Can Enhance the in Vitro Innate and Late-stage T-cell Immune Responses. Journal of Biological Chemistry, 2012. 287(30): p. 25266-25279.

46. Weltzien, R.B. and J.S. Pachter, Visualization of beta-amyloid peptide (A beta) phagocytosis by human mononuclear phagocytes: Dependency on $A$ beta aggregate size. Journal of Neuroscience Research, 2000. 59(4): p. 522-527.

47. Mohamed, A. and E. Posse de Chaves, Abeta internalization by neurons and glia. Int J Alzheimers Dis, 2011. 2011: p. 127984.

48. Lai, A.Y. and J. McLaurin, Clearance of amyloid-beta peptides by microglia and macrophages: the issue of what, when and where. Future Neurol, 2012. 7(2): p. 165-176.

49. Biancalana, M. and S. Koide, Molecular mechanism of Thioflavin-T binding to amyloid fibrils. Biochimica Et Biophysica Acta-Proteins and Proteomics, 2010. 1804(7): p. 1405-1412.

50. Reinke, A.A. and J.E. Gestwicki, Insight into Amyloid Structure Using Chemical Probes. Chemical Biology \& Drug Design, 2011. 77(6): p. 399-411.

51. Bolognesi, B., et al., ANS Binding Reveals Common Features of Cytotoxic Amyloid Species. Acs Chemical Biology, 2010. 5(8): p. 735-740.

52. Kawaguchi, H., et al., Phagocytosis of Latex-Particles by Leukocytes .1. Dependence of Phagocytosis on the Size and Surface-Potential of Particles. Biomaterials, 1986. 7(1): p. 61-66.

53. Simon, S.I. and G.W. Schmidschonbein, Biophysical Aspects of Microsphere Engulfment by Human-Neutrophils. Biophysical Journal, 1988. 53(2): p. 163-173.

54. Torche, A.M., et al., PLGA microspheres phagocytosis by pig alveolar macrophages: Influence of poly(vinyl alcohol) concentration, nature of loaded-protein and copolymer nature. Journal of Drug Targeting, 2000. 7(5): p. 343-354.

55. Sahoo, S.K., et al., Residual polyvinyl alcohol associated with poly (D,L-lactide-co-glycolide) nanoparticles affects their physical properties and cellular uptake. Journal of Controlled Release, 2002. 82(1): p. 105-114. 


\section{Supplementary materials and methods}

\section{- $\quad$ Lipopolysaccharide (LPS) detection}

The possible presence of the endotoxin LPS in protein samples was analysed using the EndoZyme ${ }^{\circledR}$ Recombinant Factor C Assay (Hyglos $\mathrm{GmbH}$, Bernried am Starnberger See, Germany) according to the manufacturer's instructions.

\section{- Solubility, pH and osmolality}

Sample solubility was calculated using the following formula:

Solubility $(\%)=\frac{\text { protein concentation }(\mathrm{mg} / \mathrm{mL}) * \text { solution volumn }(\mathrm{mL})}{\text { starting protein amount }(\mathrm{mg})} * 100 \%$

$\mathrm{pH}$ and osmolality of protein samples were measured with an Orion Star ${ }^{\mathrm{TM}} \mathrm{A} 121 \mathrm{pH}$ Portable Meter (Thermo Fischer, Waltham, Massachusetts, USA) and Osmomat 030 (Gonotec GmbH, Berlin, Germany) respectively.

\section{- THP-1 macrophage cellular uptake of fluorescent beads}

Fluoresbrite ${ }^{\circledR}$ YG Carboxylate Microspheres $2.0 \mu \mathrm{m}$ (Polysciences, Warrington, Pennsylvania, USA) was diluted in non-heated human serum to a concentration of $1 \times 10^{5}$ containing $0.001,0.01,0.1,1,10,100$ or $1000 \mathrm{ng} / \mathrm{ml}$ LPS. MO THP-1 macrophage was cultivated as indicated in the main text and $2 \mu \mathrm{L}$ of fluorescent beads dilution was added to each well. Cells were harvested after incubation for 10, 30, $60,120,240$ or 360 minutes and measured MFI by flow cytometer using same protocol of BLG sample uptake.

\section{- Circular Dichroism (CD)}

Protein samples were diluted in sodium phosphate buffer containing $0.1 \mathrm{mM}$ EDTA to a concentration of $0.15 \mathrm{mg} / \mathrm{mL}$ and measured by a Jasco J-715 spectropolarimeter (Jasco, Tokyo, Japan). Ellipticity (mdeg) was recorded within the far UV range from 185 to $260 \mathrm{~nm}$. All samples were measured 10 times in quartz cuvettes with a path length of $0.1 \mathrm{~cm}$ at $20^{\circ} \mathrm{C}$. Values were averaged and corrected for buffer background afterwards.

\section{- Size Exclusion Chromatography (SEC)}

Samples were lyophilised and re-dissolved in $200 \mu \mathrm{L}$ sodium phosphate buffer containing $150 \mathrm{mM} \mathrm{NaCl}$ to achieve a protein concentration of $10 \mathrm{mg} / \mathrm{mL}$, based on Dumas results. A system of Superdex 200 Increase 10/300 GL column on an Äktamicro machine (GE healthcare, Chicago, Illinois, USA) was injected with $50 \mu \mathrm{L}$ sample at flow rate of $0.750 \mathrm{~mL} / \mathrm{min}$. 


\section{- Correlation coefficient analysis}

Software R 3.4.2 and package "corrplot" were used to calculate a Pearson correlation coefficient and generate a correlogram (type = "lower") for visualizing the correlation matrix.

\section{Supplementary data}

Table 3.S1 LPS contamination in BLG samples

\begin{tabular}{lc}
\hline Samples & LPS ng/0.1 mg protein \\
\hline BLG & 0.1550 \\
W-BLG & 0.0004 \\
W-glu-BLG & 0.0023 \\
W-gal-BLG & 0.0010 \\
W-fru-BLG & 0.0004 \\
W-lac-BLG & 0.0077 \\
W-mal-BLG & 0 \\
W-md-BLG & 0.0038 \\
W-FOS-BLG & 0 \\
W-GOS-BLG & 0.0006 \\
H-BLG & 0.0041 \\
H-glu-BLG & 0.0046 \\
H-gal-BLG & 0.0089 \\
H-fru-BLG & 0.0023 \\
H-lac-BLG & 0.0318 \\
H-mal-BLG & 0.0274 \\
H-md-BLG & 0.0558 \\
H-FOS-BLG & 0.0438 \\
H-GOS-BLG & 0.0152 \\
L-BLG & 0.0040 \\
L-glu-BLG & 0.0078 \\
L-gal-BLG & 0.0080 \\
L-fru-BLG & 0.0045 \\
L-lac-BLG & 0.0166 \\
L-mal-BLG & 0.0034 \\
L-md-BLG & 0.0082 \\
L-FOS-BLG & 0.0102 \\
L-GOS-BLG & 0.0044 \\
\hline
\end{tabular}


Table 3.S2 Fluorescence intensity (a.u. in mean value \pm SD) of FITC-labelled protein sample for uptake experiment $(\mathrm{N}=4)$

\begin{tabular}{ll}
\hline sample & Fluorescence intensity (a.u.) \\
\hline BLG & $5428 \pm 4770$ \\
W-BLG & $14451 \pm 19996$ \\
W-glu-BLG & $7294 \pm 3762$ \\
W-gal-BLG & $6022 \pm 4098$ \\
W-fru-BLG & $8805 \pm 6915$ \\
W-lac-BLG & $7419 \pm 7414$ \\
W-mal-BLG & $5982 \pm 3200$ \\
W-md-BLG & $7029 \pm 4853$ \\
W-FOS-BLG & $7421 \pm 5562$ \\
W-GOS-BLG & $6546 \pm 4564$ \\
H-BLG & $21028 \pm 25218$ \\
H-glu-BLG & $6962 \pm 5517$ \\
H-gal-BLG & $8317 \pm 7700$ \\
H-fru-BLG & $10793 \pm 7757$ \\
H-lac-BLG & $8542 \pm 6172$ \\
H-mal-BLG & $6865 \pm 2722$ \\
H-md-BLG & $8382 \pm 4732$ \\
H-FOS-BLG & $10065 \pm 8951$ \\
H-GOS-BLG & $8869 \pm 7731$ \\
L-BLG & $21677 \pm 14161$ \\
L-glu-BLG & $12925 \pm 7525$ \\
L-gal-BLG & $10176 \pm 4832$ \\
L-fru-BLG & $18846 \pm 13023$ \\
L-lac-BLG & $11808 \pm 7921$ \\
L-mal-BLG & $13864 \pm 7891$ \\
L-md-BLG & $17012 \pm 9783$ \\
L-FOS-BLG & $26942 \pm 21644$ \\
L-GOS-BLG & $18505 \pm 14635$ \\
\hline & \\
\hline
\end{tabular}


A

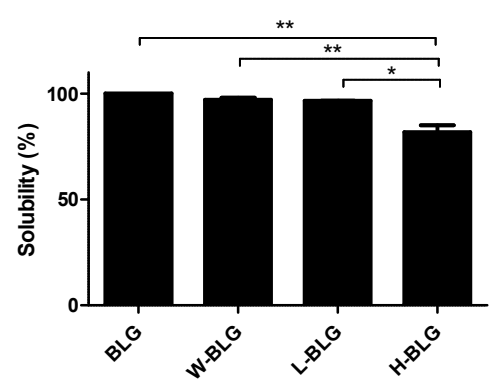

C

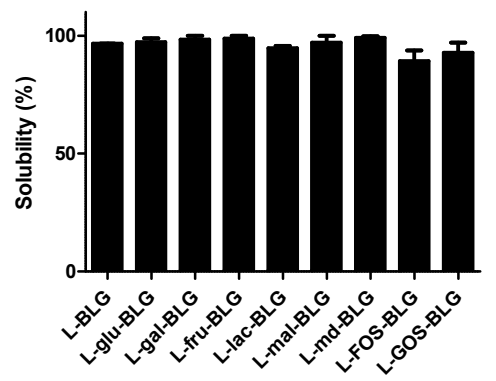

B
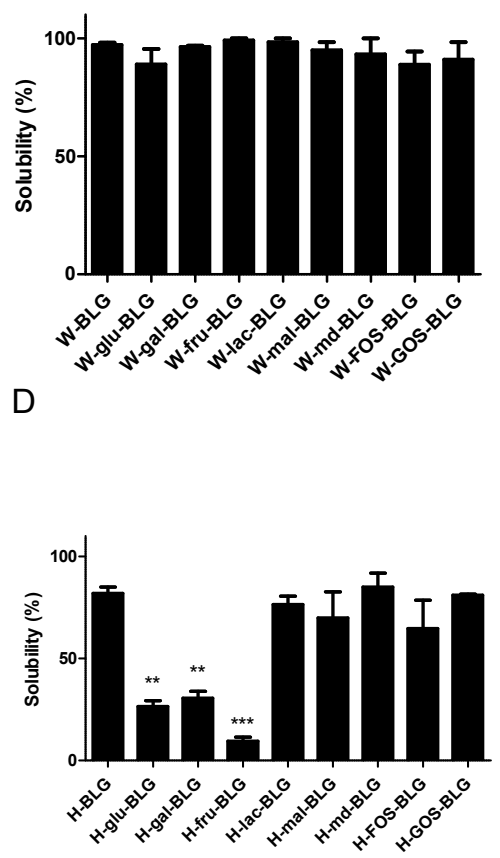

Figure 3.S1 High temperature dry heating, especially in presence of monosaccharides, reduced the solubility of BLG. BLG was non-treated or heated $(W, L$ or $H)$ in absence or presence of saccharides (glu, gal, fru, lac, mal, md, FOS or GOS). The solubility of non-treated BLG or BLG heat-treated in absence of sugars $(A)$ and wet-heated $(B)$, low-temperature dry-heated $(C)$ and high-temperature dry-heated (D) BLG in presence of saccharides was determined by DUMAS. Bars represent mean value \pm SEM of 2 independent sample sets. Statistical differences were calculated with Tukey's Multiple Comparison Test (A) or Dunnett's Test (B-D): * ${ }^{*}<0.05 ;{ }^{*} p<0.01 ;{ }^{* *} p<0.001$. 
A

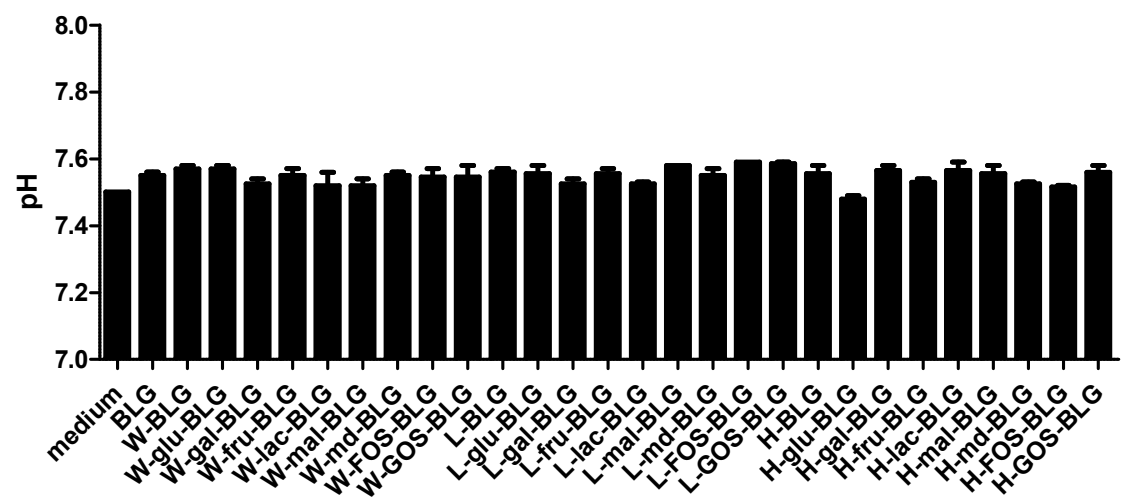

B

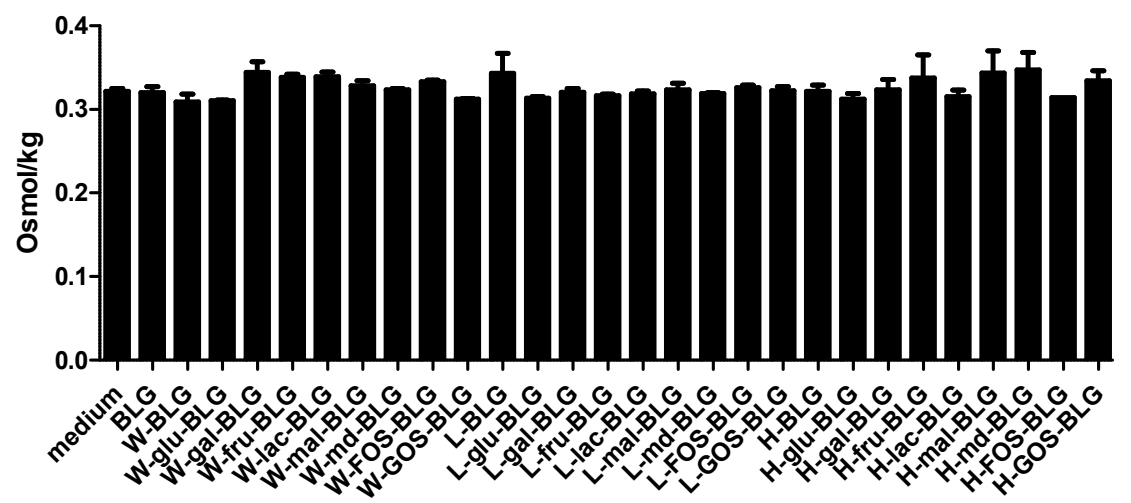

Figure 3.S2 The $\mathrm{pH}$ or osmolality of treated BLG dissolved in medium were similar to native BLG. BLG was non-treated or heated (W, L or H) in absence or presence of saccharides (glu, gal, fru, lac, mal, md, FOS or GOS). The $\mathrm{pH}$ and osmolality in non-treated BLG or BLG heat-treated in absence of sugars $(A)$ and wet-heated (B), low-temperature dry-heated (C) and high-temperature dry-heated (D) BLG in presence of saccharides were determined with a $\mathrm{pH}$ meter and Osmomat, respectively. Bars represent mean value \pm SEM of 2 independent sample sets. No statistical significant differences were detected. 


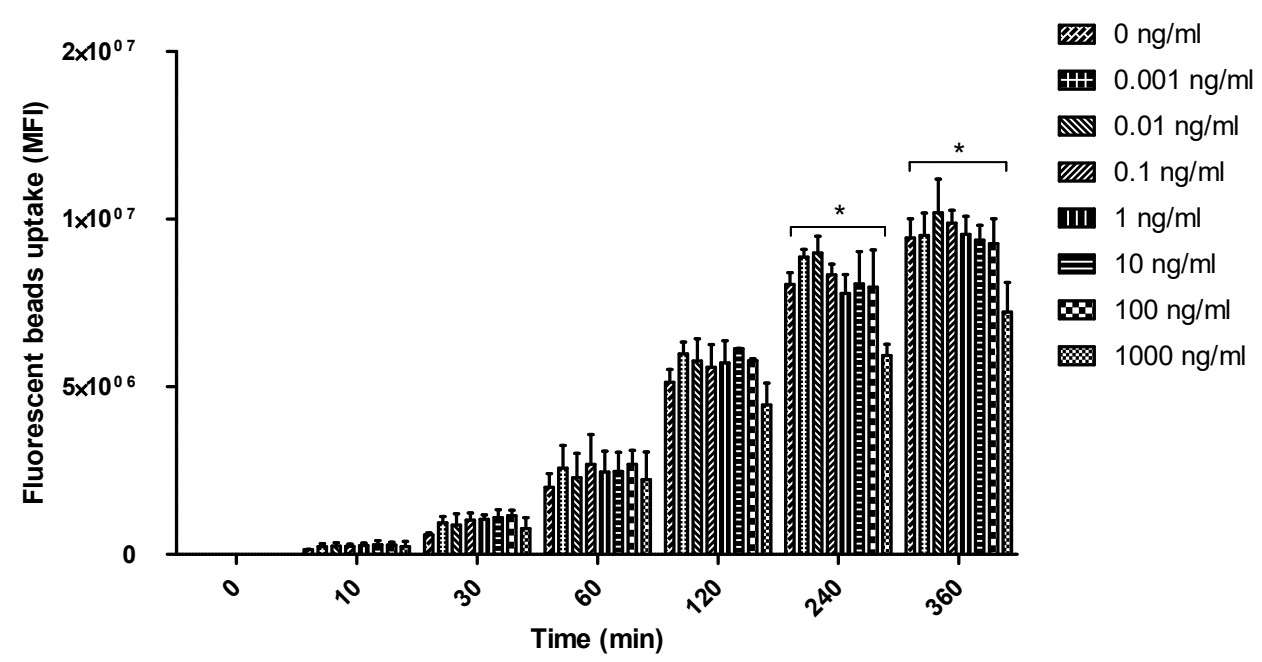

Figure 3.S3 LPS did not influence the uptake of beads by THP-1 macrophages with an incubation period of 120 minutes or below $1000 \mathrm{ng} / \mathrm{ml}$ of LPS. The uptake of fluorescent beads by THP-1 macrophages following stimulation with $0.001,0.01,0.1,1,10,100 \mathrm{or} 1000 \mathrm{ng} / \mathrm{ml}$ of lipopolysaccharide for 10, 30, $60,120,240$ or 360 minutes was determined by flow cytometry as mean fluorescence intensity (MFI). Bars represent mean value $\pm S D$ of 2 independent measurements. Statistical differences were calculated with a two-way repeated measures ANOVA test: ${ }^{*} p<0.05$. 
A

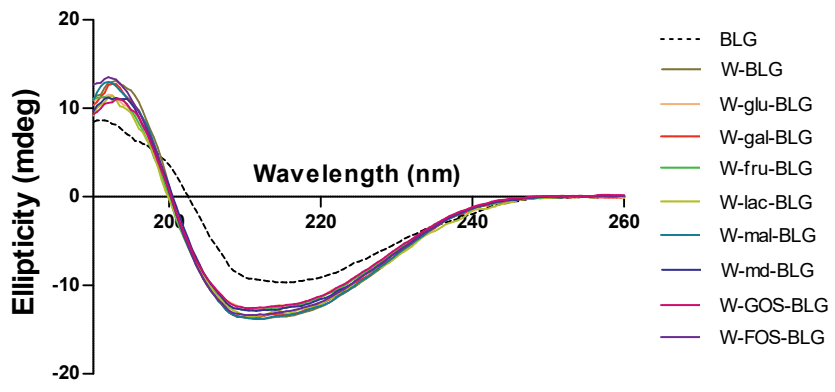

B
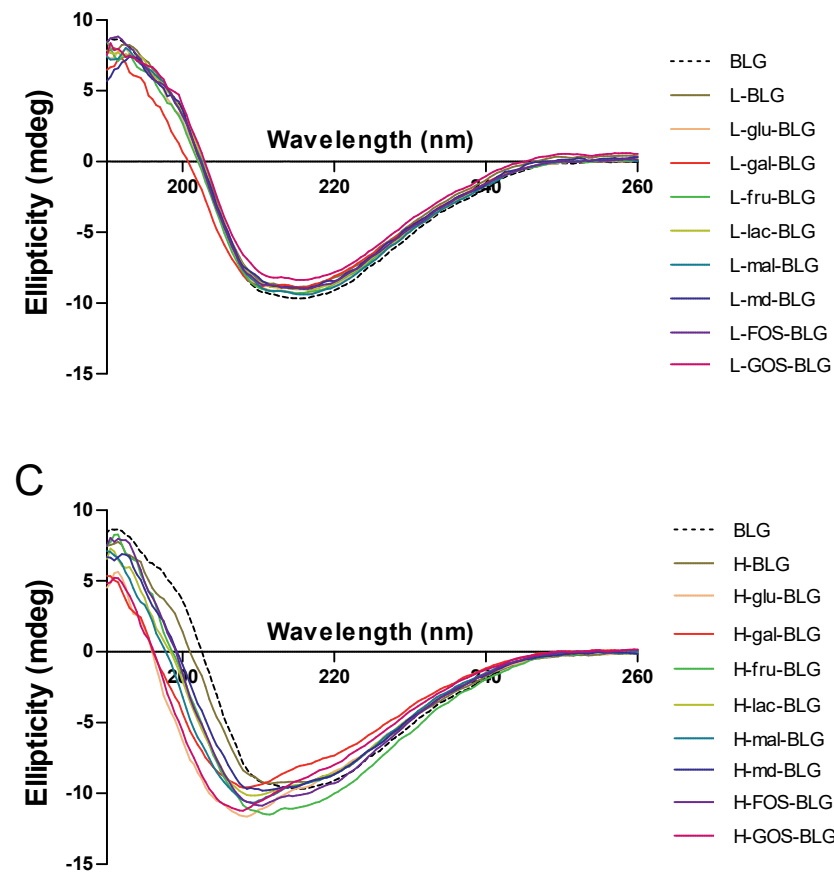

Figure 3.S4 Heating methods and/or the presence of saccharides can alter the secondary structure of BLG. BLG was non-treated or heated (W, L or H) in absence or presence of saccharides (glu, gal, fru, lac, mal, md, FOS or GOS). The far UV protein spectrum of wet-heated (A), low-temperature dry-heated (B) and high-temperature dry-heated (C) BLG in presence of saccharides and non-treated BLG were determined with circular dichroism. Lines represent mean value of duplicate measurements each averaging 10 recordings. 

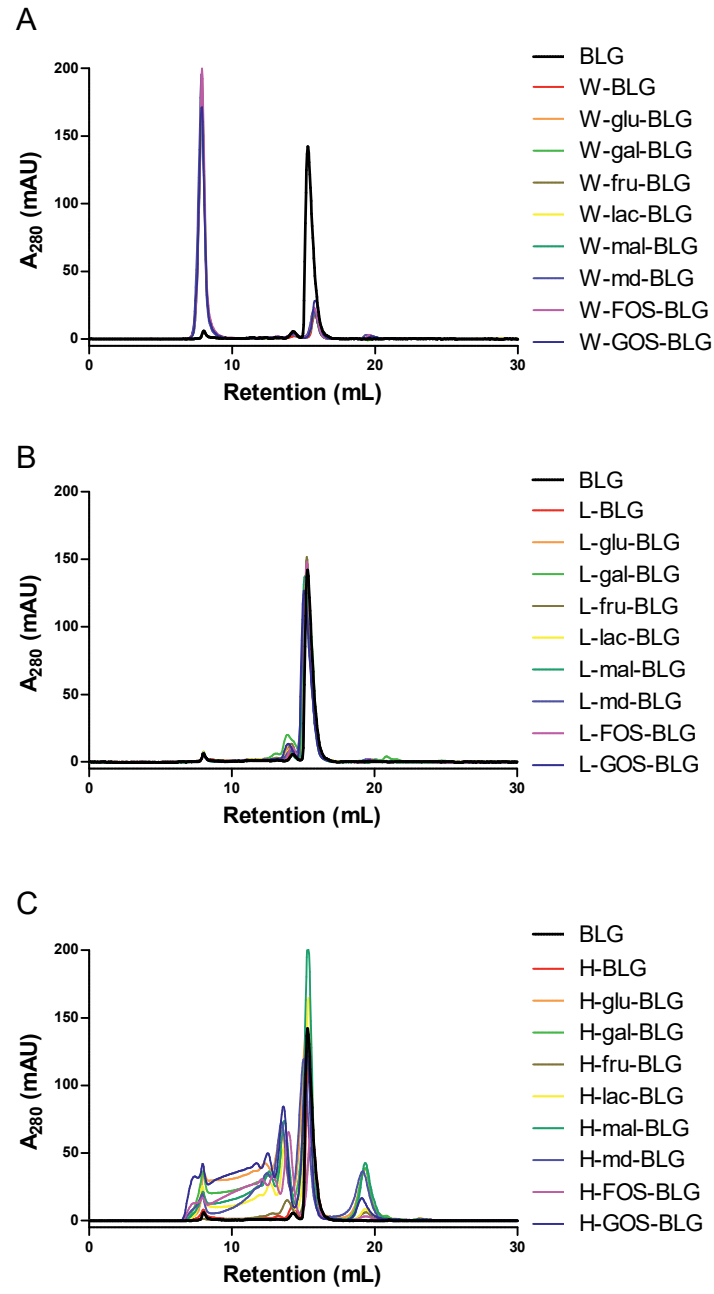

Figure 3.55 Heating methods and/or the presence of saccharides can alter the molecular weight distribution of BLG. BLG was non-treated or heated (W, $L$ or $H)$ in absence or presence of saccharides (glu, gal, fru, lac, mal, md, FOS or GOS). The molecular weight distribution of wet-heated (A), lowtemperature dry-heated (B) and high-temperature dry-heated $(C) B L G$ in presence of saccharides and non-treated BLG were determined with SEC chromatography. 
A

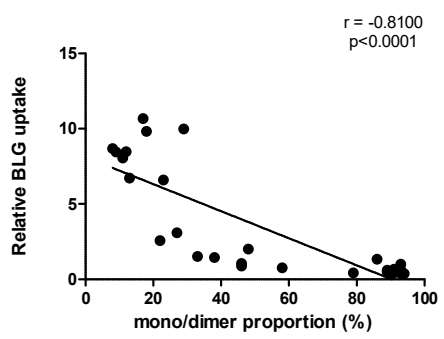

C

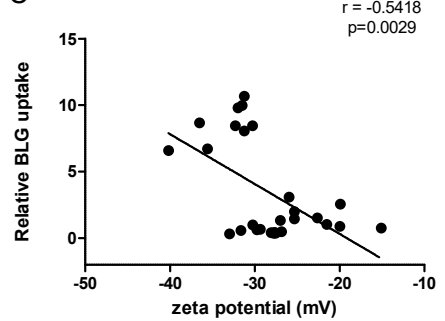

$\mathrm{E}$

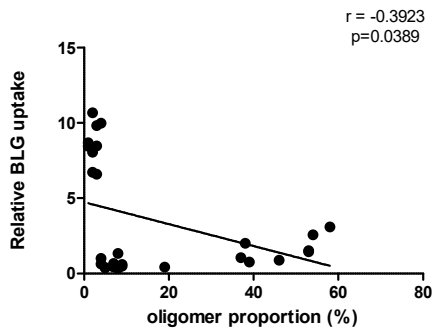

G

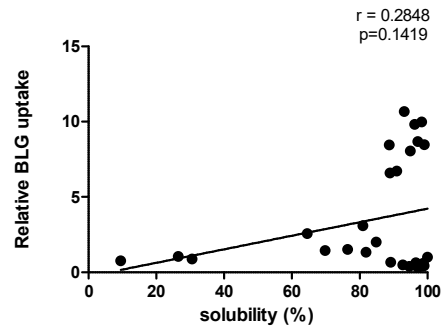

B

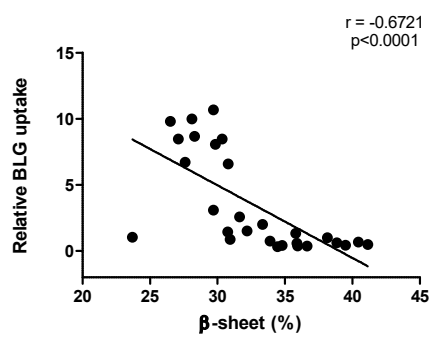

D

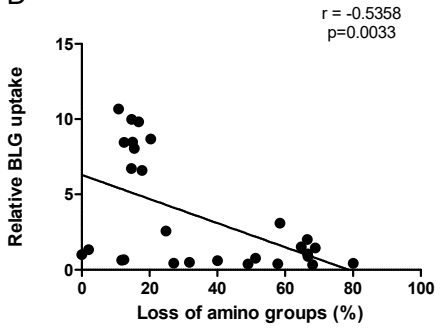

$\mathrm{F}$

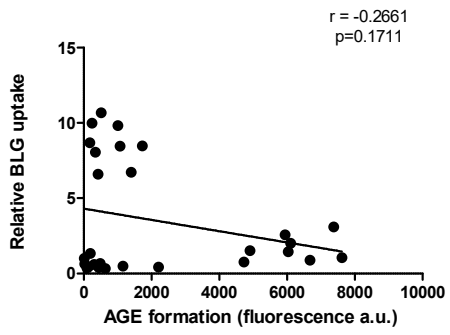

$\mathrm{H}$

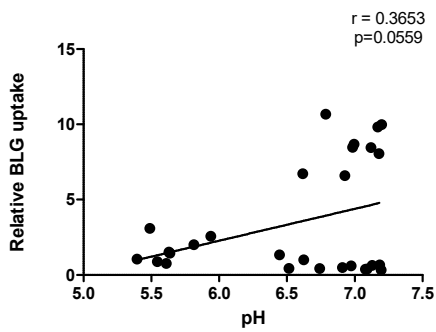

Figure 3.S6 Uptake of BLG by THP-1 macrophage moderately correlates with percentage of 6 -sheet, surface charge and loss of free amino groups. Uptake of thermally treated BLG in the presence or absence of saccharides was strongly corrected with monomer/dimer proportion, moderate correlated with percentage of 6 -sheet, zeta-potential, loss of free amino group amyloid-like structure with an absolute $r$ value between 0.5 and 0.8 . No clear correlation was found between uptake and oligomer proportion, AGE formation, solubility and $\mathrm{pH}$. 


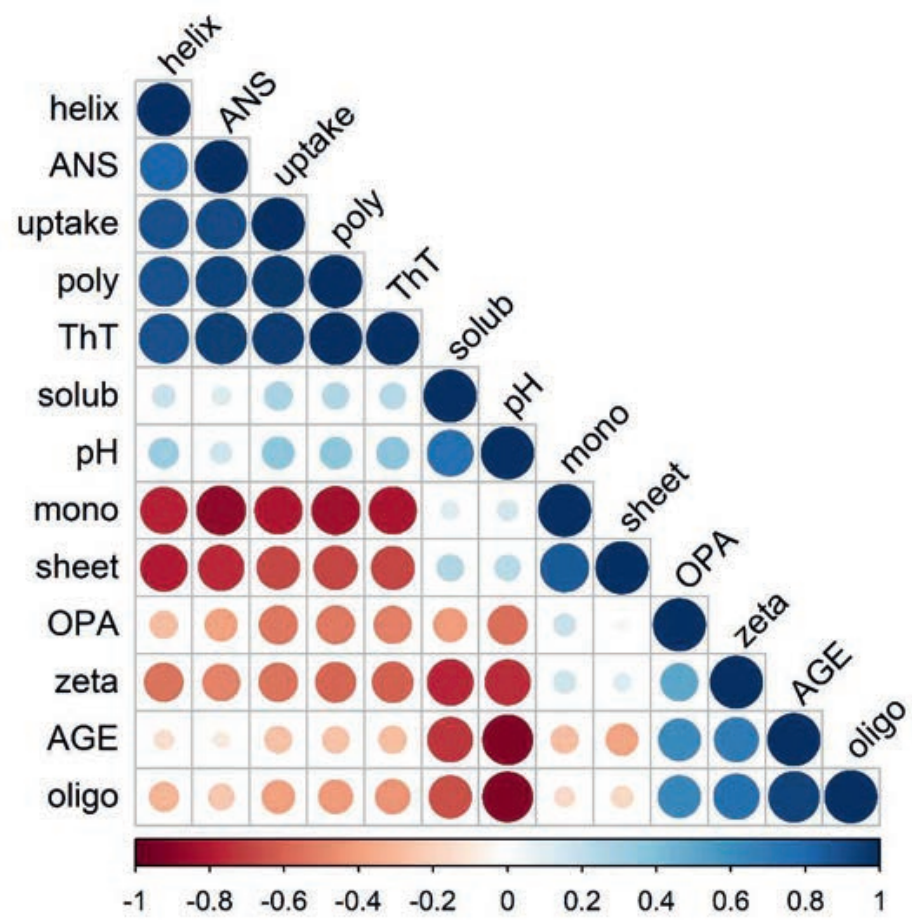

Figure 3.S7 Uptake of BLG by THP-1 macrophage correlates with the percentage of $\alpha$-helix, hydrophobicity, aggregation and amyloid-like structure presence. Thermally treated BLG in the presence or absence of saccharides was tested for solubility, $\mathrm{pH}$, uptake by THP-1 macrophages (uptake), AGE formation (AGE), glycation (OPA), amyloid-like structure formation (ThT), percentage of $\alpha$-helix (helix) or 6 -sheet (sheet), aggregation (monomer and dimer (mono), oligomers (oligo), polymers (poly)), exposure of hydrophobic regions (ANS) and surface charge (zeta). Correlation between these parameters was determined using R-programming language and the "corrplot" package. 



\section{Chapter 4}

\section{A THP-1 Cell Line-based Exploration of Immune Responses Towards Heat- treated BLG}

Ying Deng, Coen Govers, Ellen ter Beest, Aalt-Jan van Dijk, Kasper Hettinga and Harry J. Wichers

Manuscript submitted 
Abstract: Allergen recognition and processing by antigen presenting cells is essential for the sensitization step of food allergy. Macrophages and dendritic cells are both phagocytic antigen presenting cells and play important roles in innate immune responses and signaling between the innate and adaptive immune system. To obtain a model system with a homogeneous genetic background, we derived macrophages and dendritic cells from THP-1 monocytes. The difference between macrophages and dendritic cells was clearly shown by the difference in transcription response (microarray) and protein expression levels. Their resemblance to primary cells was analyzed by comparison to properties as described in literature. The uptake of $\beta$-lactoglobulin after wet-heating ( $60{ }^{\circ} \mathrm{C}$ in solution) by THP-1 macrophages was reported to be significantly increased. To analyse the subsequent immune response, we incubated THP-1 derived macrophages and dendritic cells with native and differently processed $\beta$-lactoglobulin and determined the transcription and cytokine expression levels of the cells. A stronger transcriptional response was found in macrophages than dendritic cells, while severely structurally modified $\beta$-lactoglobulin induced a limited transcriptional response, especially when compared to native and limitedly modified $\beta$-lactoglobulin. These results showed that that processing is relevant for the transcriptional response towards $\beta$-lactoglobulin of innate immune cells and also that the transcriptional response was not related to cellular uptake efficiency. 


\section{Introduction}

A food allergy is an immune mediated reaction against an otherwise harmless protein, also known as allergen $[1,2]$. Cow's milk allergy is one of the most common food allergies in the world which affects about $2.5 \%$ of all children [3]. Thermal processing of milk is an essential step in the dairy industry to ensure microbiological safety and prolong shelf life [4]. However, heat treatments like pasteurization, sterilization and spray drying will affect the structural characteristics of the proteins present in cow's milk, including the whey protein $\beta$-lactoglobulin (BLG) which is one of the major allergens present in milk [5-7]. Structural modifications like unfolding, aggregation and glycation with lactose could occur during the heat treatment, either reducing or enhancing the allergenic potential, i.e. IgE-binding, of $\operatorname{BLG}[8,9]$.

After passing through the epithelial cells of the intestine, BLG may be taken up by antigen-presenting cells (APC) of the innate immune system. Of these, macrophages and dendritic cells are considered to be professional APCs [10]. They have the ability to capture an allergen into specialised endosomes/lysosomes, and then process it into linear peptides which are expressed on major histocompatibility complex (MHC) molecules on the outer surface, to which a naïve T cell can bind. Although both derived from the same precursor-monocytes, macrophages and dendritic cells exert different functions and functional properties. Macrophages focus on scavenging allergens, manipulating inflammatory responses and recruiting $T$ cells and B cells in situ. Dendritic cells are specialized in their ability to preserve the ingested allergens, driving $\mathrm{T}$ cell responses following their migration to the secondary lymphoid tissues [11].

To study the immunological response of macrophages and dendritic cells upon exposure to heattreated BLG, macrophages and dendritic cells derived from THP-1 cells were used. This approach would circumvent possible effects of different genetic backgrounds on the cellular responses [12] thus provides a more reproducible picture of the effect of processing of proteins. THP-1 monocytes stimulated by phorbol-12-myristate-13-acetate (PMA) are regarded as macrophage-like cells, showing similarities with primary macrophages in morphology, gene transcription and expression [13-16]. There are only few reports describing the differentiation of THP-1 monocytes into dendritic-like cells. A recent study showed THP-1 monocytes, stimulated with PMA together with IL-4, to express typical cellular surface markers and properties of primary immature dendritic cells [17].

By using microarray analysis, the similarities and differences between THP-1 derived macrophages and dendritic cells were measured based on the transcription levels for their entire genome. Together with morphology, protein expression levels, and antigen processing capability analyses, their resemblance 
to primary cells was estimated according to the reported properties of primary cells from literature [18]. Differences in uptake capability of THP-1 derived macrophage-like and dendritic-like cells upon exposure to differently heat-treated BLG was described in Chapter 3 and 5. Finally, the immune reactivity of THP-1 derived APCS stimulated by differently heat-treated BLG was analysed in this study.

\section{Materials and methods}

\section{Chemicals}

All chemicals were purchased from Sigma Aldrich (St Louis, Missouri, USA) unless otherwise stated.

\section{THP-1 cell culture and differentiation}

The human monocytic leukemia cell line THP-1 (ATCC, Manassas, Virginia, USA) was differentiated into cells characteristic for resting macrophages (MO) as described by Chanput et al. [19] at a concentration of $1 \times 10^{6}$ cells $/ \mathrm{mL}$ using $100 \mathrm{ng} / \mathrm{mL}$ phorbol-12-myristate-13-acetate (PMA) for a 48 hours stimulation followed by 2 times cell washing with medium and another 48 hours of resting. Similarly, using a cell concentration of $0.25 \times 10^{6}$ cells $/ \mathrm{mL}$, immature dendritic cells (iDC) were generated after incubating with $20 \mathrm{ng} / \mathrm{mL}$ of IL-4 (R\&D Systems, Minneapolis, Minnesota, USA) and $20 \mathrm{ng} / \mathrm{mL}$ of PMA for 4 days as described by Katayama et al. [20]. Lipopolysaccharide (LPS) was used to stimulate MO and iDC into LPS-M and mature dendritic cells (mDC) with a concentration of $500 \mathrm{ng} / \mathrm{mL}$ and $1 \mu \mathrm{g} / \mathrm{mL}$, respectively, for 48 hours.

\section{Morphology measurement of cells}

The morphology of cells was observed by an automated digital inverted microscope Evos FL Auto 2 (Thermo Fisher, Waltham, Massachusetts, USA) using a 40x objective lens. Scale bars were added to the picture using ImageJ software.

\section{Sample preparation and cell stimulation}

$\beta$-Lactoglobulin (BLG) was isolated from raw cow's milk as described in the protocol of De Jongh et al. [21]. In the absence or presence of glucose, BLG was wet-heated (W), high-temperature dry-heated (H) or low-temperature dry-heated (L), centrifuged, dialyzed and the protein concentration measured, as described previously (Chapter 3). THP-1 derived MO and iDC were incubated in Corning ${ }^{\circledR} 24$ Well TCTreated Microplates (Life Sciences, Tewksbury, Massachusetts, USA) with $100 \mu \mathrm{g} / \mathrm{mL}$ BLG, L-glu-BLG, H-glu-BLG, W-glu-BLG and the culture medium as a control for 6 hours. 


\section{Microarray analysis}

THP-1 derived MO and IDC with or without exposure to BLG samples were incubated in Corning ${ }^{\circledR} 24$ Well TC-Treated Microplates (Life Sciences, Tewksbury, Massachusetts, USA). RNA of the cultured cells was extracted as described by Tang et al. [22], using $200 \mu \mathrm{L}$ TRIzol (Invitrogen, Bleiswijk, The Netherlands) per well. Each cell experiment was performed in triplicate with cells from different passage numbers. RNA quality was verified using the RNA 6000 Nano assay on an Agilent 2100 Bioanalyzer (Agilent Technologies, Amstelveen, The Netherlands). Quality approved RNA was labelled and hybridized to the GeneChip ${ }^{\circledR}$ Human Gene 2.1 ST Array in a GeneTitan ${ }^{\circledR}$ platform (Affymetrix, Santa Clara, California, USA) according to standard protocols. The arrays were scanned by the high-resolution scanner of GeneChip Scanner (Affymetrix, Santa Clara, California, USA) and the signal was converted to cell intensity file (.CEL files) using GeneChip Operating Software (GCOS). The raw CEL data was normalized using the Bioconductor packages of MADMAX pipeline [23] and analysed after data filtering. Fold change (FC) of MO and IDC was calculated based on the gene transcription level compared to monocytes, while FC of $\mathrm{MO}$ or IDC incubated with BLG samples was compared with MO or IDC without incubation. Genes with an absolute FC value $>2$ and an intensity-based moderated t-statistics (IBMT) raw q-value $<0.05$ were considered to be significantly differentially transcribed.

\section{Data visualization}

The Venn diagrams were generated using Venny 2.1 (https://bioinfogp.cnb.csic.es/tools/venny/).

\section{Pathway analysis}

Pathway analysis was carried out using the program Ingenuity Pathway Analysis (IPA, Qiagen, Hilden, Germany). For $\mathrm{MO}$ and $\mathrm{BDC}$ without BLG incubation and $\mathrm{MO}$ incubated with native and low-temperature dry-heated BLG, only the significantly differentially transcribed genes were uploaded in the software. The IPA core analysis was done based on setting: "Human" in the "Species" category, and "THP1 ", "Dendritic cells" or "Macrophages" in the category of "Tissue \& Cell lines".

\section{Cell staining and flow cytometry analysis}

Cells cultivated in Corning ${ }^{\circledR} 96$ Well TC-Treated Microplates (Life Sciences, Tewksbury, Massachusetts, USA) were harvested as described previously (Chapter 3 ). Cell surface marker staining was done by first suspending the cell pellets with $100 \mu \mathrm{L}$ PBS containing $10 \mu \mathrm{L}$ FcR blocking reagent (Miltenyi Biotec, Auburn, CA) and incubating the cell suspensions at $4{ }^{\circ} \mathrm{C}$ for $10 \mathrm{~min}$. Then $90 \mu \mathrm{L}$ PBS containing $1 \mu \mathrm{L}$ of each detection antibody was added to the cells and incubated for $30 \mathrm{~min}$ at $4{ }^{\circ} \mathrm{C}$ (gently mixed in 
between once). The following fluorescence-labelled mouse anti-human monoclonal antibodies (mAbs) were used: panel 1 [FITC-conjugated CD209 (clone DCN46), PE-conjugated HLA-DR (clone G46-6), PE$\mathrm{Cy}^{\mathrm{TM}} 7$-conjugated CD83 (clone HB15e) and APC-conjugated CD11c (clone B-ly6)], panel 2 [FITC-

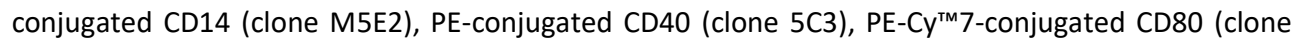
L307.4) and APC-conjugated HLA-ABC (clone G46-2.6)] and panel 3 [FITC-conjugated CD63 (clone H5C6) and APC-conjugated CD81 (clone JS-81)] all from BD Biosciences (Franklin Lakes, New Jersey, USA). After staining, $1 \mathrm{~mL}$ PBS was added to the cells followed by centrifugation for 5 minutes at $450 \times \mathrm{g}$. The cell pellet was resuspended in $150 \mu \mathrm{L}$ PBS and the mean fluorescence intensity (MFI) of each fluorescence signal was analyzed for four-color immunofluorescence with an Accuri C6 flow cytometry (BD Biosciences, Franklin Lakes, New Jersey, USA) of at least 5,000 cells for each sample using BD Accuri C6 software. The MFI of unstained cells was subtracted from each value.

\section{Cytokine production measurement}

Supernatant of THP-1 derived MO and IDC without sample stimulation or incubated for 16 hours with $100 \mu \mathrm{g} / \mathrm{mL}$ samples were collected. The cytokine production measurement was done with the ELISA Deluxe Set Human IL-8/IL10/IL-6/TNF- $\alpha /$ CCL20/IL-1ß (Biolegend, San Diego, California, United States) following the manufacturer's protocol.

\section{Assessing the antigen processing capacity of cells using DQ-ovalbumin (DQ-OVA)}

DQ-OVA is a self-quenched conjugate of ovalbumin which will exhibit bright green fluorescence upon proteolytic degradation. THP-1 derived MO or iDC cells in Corning ${ }^{\circledR} 96$ Well TC-Treated Microplates (Life Sciences, Tewksbury, Massachusetts, USA) were incubated with medium containing $10 \mathrm{ug} / \mathrm{mL}$ DQOVA (Life Sciences, Tewksbury, Massachusetts, USA) for 1 hour. After that, cells were washed twice with PBS without $\mathrm{Ca}^{2+}$ and $\mathrm{Mg}^{2+}$ (Gibco, Thermo Fisher, Waltham, Massachusetts, USA) and incubated with $100 \mu \mathrm{L}$ of $0.25 \%$ Trypsin-EDTA (Gibco, Thermo Fisher, Waltham, Massachusetts, USA) for 5 minutes. The cell suspension in Trypsin-EDTA was centrifuged for 5 minutes at $450 \times \mathrm{g}$, after which the cell pellets were resuspended in $150 \mu \mathrm{L}$ PBS. The mean fluorescence intensity (MFI) of the FITC signal was recorded by flow cytometry (Accuri C6, BD Biosciences, Franklin Lakes, New Jersey, USA) of at least 5,000 cells for each sample using BD Accuri C6 software.

\section{Statistics}

The statistical analysis of microarray data was performed as described in the previous paragraph. The statistical analysis of other data was done using Prism 6 software or R studio (package "agricolae"), considering $\mathrm{p}<0.05$ to be significant. 


\section{Results}

\section{THP-1 monocyte-derived macrophages and dendritic cells differed in morphology}

To build genetically homogenous innate immune cell models, we used the THP-1 monocytic cell line to obtain macrophage-like and dendritic-like cells. After 2 days of culturing in medium supplemented with $100 \mathrm{ng} / \mathrm{mL}$ of PMA followed by 2 days of rest or 4 days with $20 \mathrm{ng} / \mathrm{ml}$ of PMA and $20 \mathrm{ng} / \mathrm{m}$ of IL-4, THP1 monocytes differentiated into macrophage-like cells in the resting stage $(\mathrm{MO})$ or immature dendriticlike cells (iDC), respectively. We first analyzed the morphology of different stimulated cells in comparison with their precursor monocytes. Morphology of both induced cell types demonstrated an increase in size while MO maintained their globosity, and iDC showed an elongated shape (Figure 4.1). In addition, both induced cell types were adherent in contrast to the monocytes.
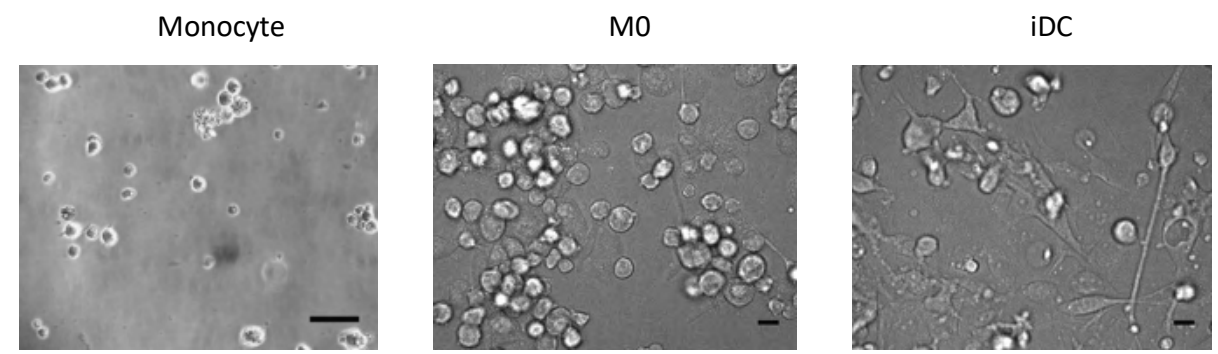

Figure 4.1 The morphologies of THP-1 monocytes and their derived macrophage-like cells at resting stage (MO) and immature dendritic-like cells (IDC) can be distinguished from each other. THP-1 monocytes were differentiated into macrophage-like and dendritic-like cells on a 12 well plate and analyzed under the microscope. Scale bar is $20 \mu \mathrm{m}$. Representative pictures of repeated differentiation experiments were shown.

\section{Gene transcription and pathway analysis of THP-1 monocyte-derived macrophages and dendritic cells showed different profiles}

The gene transcription of $\mathrm{MO}$ and $\mathrm{DCC}$ was compared qualitatively and quantitatively to that of monocytes to obtain an indication of up- or down-regulation of specific macrophage and dendric cell related genes. In total, there were 5,307 genes transcribed significantly differentially in at least one of the cell types out of more than 25,000 tested genes. As shown in the scatter plot of the significantly differentially transcribed genes (Figure 4.2A), the correlation of the transcription fold change of MO and iDC was weak $(r=0.645, p<0.001)$. Moreover, it can be observed in Figure 4.2B that approximately half of the significantly differentially transcribed genes were shared by both cell types, but these two 
cell types also showed unique transcription profiles. A detailed list with 25 genes which showed the largest transcription difference in MO and iDC cells can be found in Table $4 . \mathrm{S} 1$ (increased transcription in $\mathrm{MO}$ ) and 4.52 (increased transcription in iDC). Notably, the overall fold change in transcription level of iDC was much higher than for M0. CYBB, IL7R, CD180, WIPF3, KCNMA1, MMP2, TLR7, ZC3H12D and CHODL demonstrated higher transcription levels in $\mathrm{MO}$ and these genes are related to antigen uptake, innate immunity and cell signalling. ENDRA, S1PR1, CHRM3, C3AR1 which also demonstrated higher transcription levels in $\mathrm{MO}$ are related to $\mathrm{G}$ protein and its coupled receptors. Four SERPINB related genes were in the gene list of iDC which might be due to the IL-4 usage for cell stimulation [24, 25]. AQP9, CCL22, INHBE, CD274, MMP7 and VCAM1 which are related to immunological response, EHF, SSCIMP, NR4A3 and ALDH1A2 which are related to dendritic cell, GJB2 and VCAM1 which are related to cell adhesion had a higher transcription level in iDC compared to MO.

A

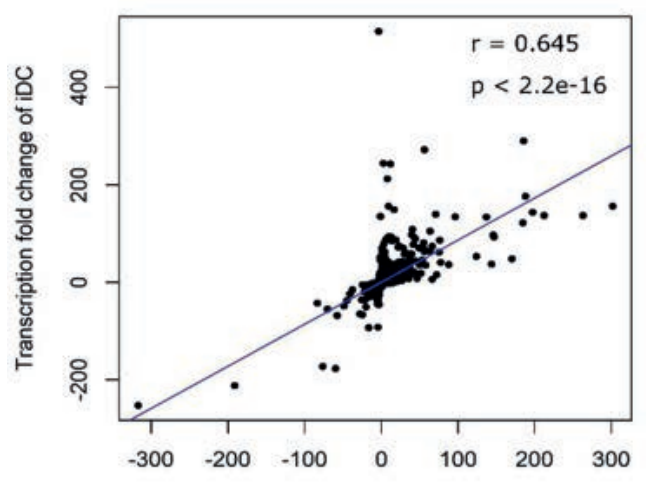

Transcription fold change of $\mathrm{MO}$

\section{B}

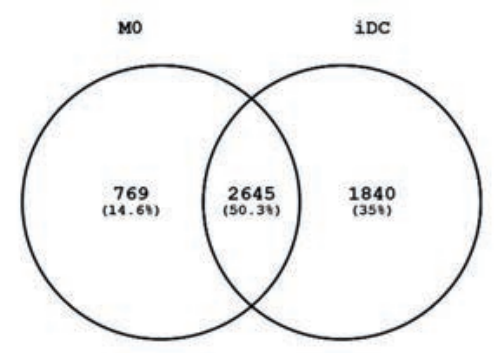

Figure 4.2 Significantly differentially transcribed genes in macrophage and dendritic cells have both similarities and differences. THP-1 monocytes were differentiated into macrophage-like and dendriticlike cells and the gene transcription level was measured by microarray. Genes with an absolute value of fold change $>2$ and $q$ value $<0.05$ in at least one cell type are considered to be significant. The scatter plot $(A)$ was generated to show the correlation of transcription level between MO and iDC whereas the Venn diagram $(B)$ showed the numbers of significantly transcribed genes which are shared or exclusive for different cell types. The Pearson correlation coefficient ( $r$ ) and two-tailed $p$ value were analyzed using $R$ studio. 


\section{Canonical Pathways}

MO iDC

Inhibition of Matrix Metalloproteases

Estrogen-mediated S-phase Entry

Cell Cycle Control of Chromosomal Replication

Role of Tissue Factor in Cancer

Role of BRCA1 in DNA Damage Response

Bladder Cancer Signaling

Hepatic Fibrosis / Hepatic Stellate Cell Activation

Glioma Invasiveness Signaling

IL-8 Signaling

Hepatic Fibrosis Signaling Pathway

Atherosclerosis Signaling

Granulocyte Adhesion and Diapedesis

Aryl Hydrocarbon Receptor Signaling

Macropinocytosis Signaling

Colorectal Cancer Metastasis Signaling

Ovarian Cancer Signaling

Paxillin Signaling

Acute Phase Response Signaling

Inhibition of Angiogenesis by TSP1

Virus Entry via Endocytic Pathways

IL-6 Signaling

Toll-like Receptor Signaling

GADD45 Signaling

Mismatch Repair in Eukaryotes

Role of CHK Proteins in Cell Cycle Checkpoint Control

Hereditary Breast Cancer Signaling

Cyclins and Cell Cycle Regulation

Leukocyte Extravasation Signaling

IL-10 Signaling

LXR/RXR Activation

Agranulocyte Adhesion and Diapedesis

ATM Signaling

Molecular Mechanisms of Cancer

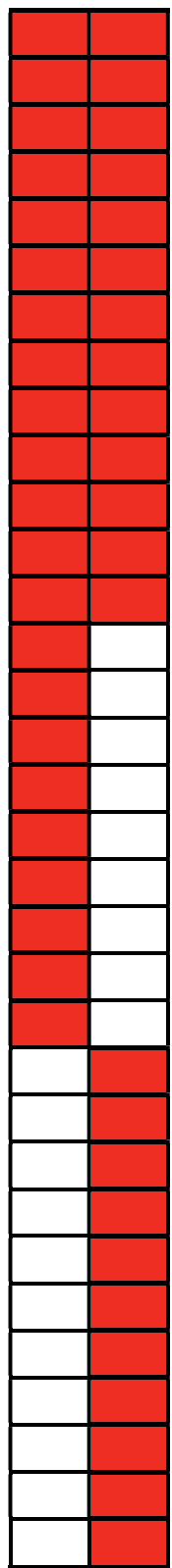

Figure 4.3 Significantly associated canonical pathway patterns in MO and iDC differed from each other. THP-1 monocytes were differentiated into macrophage-like (MO) or dendritic-like cells (iDC) and gene transcription levels were imported into IPA for canonical pathway analysis. Canonical pathways with a $p$ value $<0.001$ were labelled in red. 
The microarray data were analysed with Ingenuity Pathway Analysis (IPA) based on the significantly differentially transcribed genes for MO and iDC. The significantly associated canonical pathways $(p<$ 0.001) are listed in Figure 4.3. Inhibition of Matrix Metalloproteases, Atherosclerosis Signaling and Aryl Hydrocarbon Receptor Signaling which are pathways related to cell differentiation or proliferation were significantly associated with both cell types, as well as IL-8 Signaling and Granulocyte Adhesion and Diapedesis which are pathways related to inflammation and immune response. Macropinocytosis Signaling and Virus Entry via Endocytic Pathways (uptake related), Paxillin Signaling (cell adhesion and morphology related), Acute Phase Response Signaling and Toll-like Receptor Signaling (proinflammatory and cytokine related) were significantly associated with MO. On the other hand, Leukocyte Extravasation Signaling, IL-10 Signaling and Agranulocyte Adhesion and Diapedesis are immune response related pathways which were significantly associated with iDC. As shown in Table 4.S3, upstream regulators ECSIT, CD40LG, TNF- $\alpha$, TLR4, CD40, IL17A, IL6 and TNFRSF1A were found to be activated in both $\mathrm{MO}$ and $\mathrm{IDC}$. However, the extent of activation was stronger in iDC than MO, with TNF- $\alpha$, TLR- 4 and TREM1 had the biggest difference in $z$-score and TSLP was activated in iDC but absent in $\mathrm{MO}$.

\section{Surface marker expression, cytokine production and antigen processing clearly distinguished THP-1 derived macrophages from dendritic cells}

To further characterize the two different cell types generated from THP-1 monocytes, based on gene expression, we measured their cell surface marker expression and secretion of cytokines. In addition, to compare cells in an inflammatory state, we challenged them with LPS, resulting in LPS-stimulated macrophages (LPS-M) and mature dendritic cells (mDC). As shown in Figure 4.4, expression of circulating monocyte-derived macrophage differentiation markers CD14 and HLA-ABC $[26,27]$ were significantly higher in MO compared to monocytes or iDC, while the expression of circulating monocyte-derived dendritic cell marker and T cell activator CD209 [28] was significantly higher in iDC compared to monocytes or M0. Primary APC surface markers CD11C and CD81 [29, 30] had similar expression levels in MO and iDC and were both significantly higher expressed than in monocytes. Furthermore, CD63 which was reportedly expressed on circulating monocyte-derived immature dendric cells [31], showed significantly higher expression in iDC but not in $\mathrm{MO}$ compared to monocytes. After LPS stimulation, expression of CD83 and CD40, which are related to dendritic cell maturation and interaction with T and B cells [32], were significantly higher in MDC than iDC or LPS-M. CD209 was significantly decreased in $\mathrm{MDC}$ compared to iDC. On the other hand, cell surface expression levels of CD14 and CD80 (activated circulating monocyte-derived macrophage marker) [33] on LPS-M were significantly higher than on both $\mathrm{MO}$ and $\mathrm{mDC}$. 

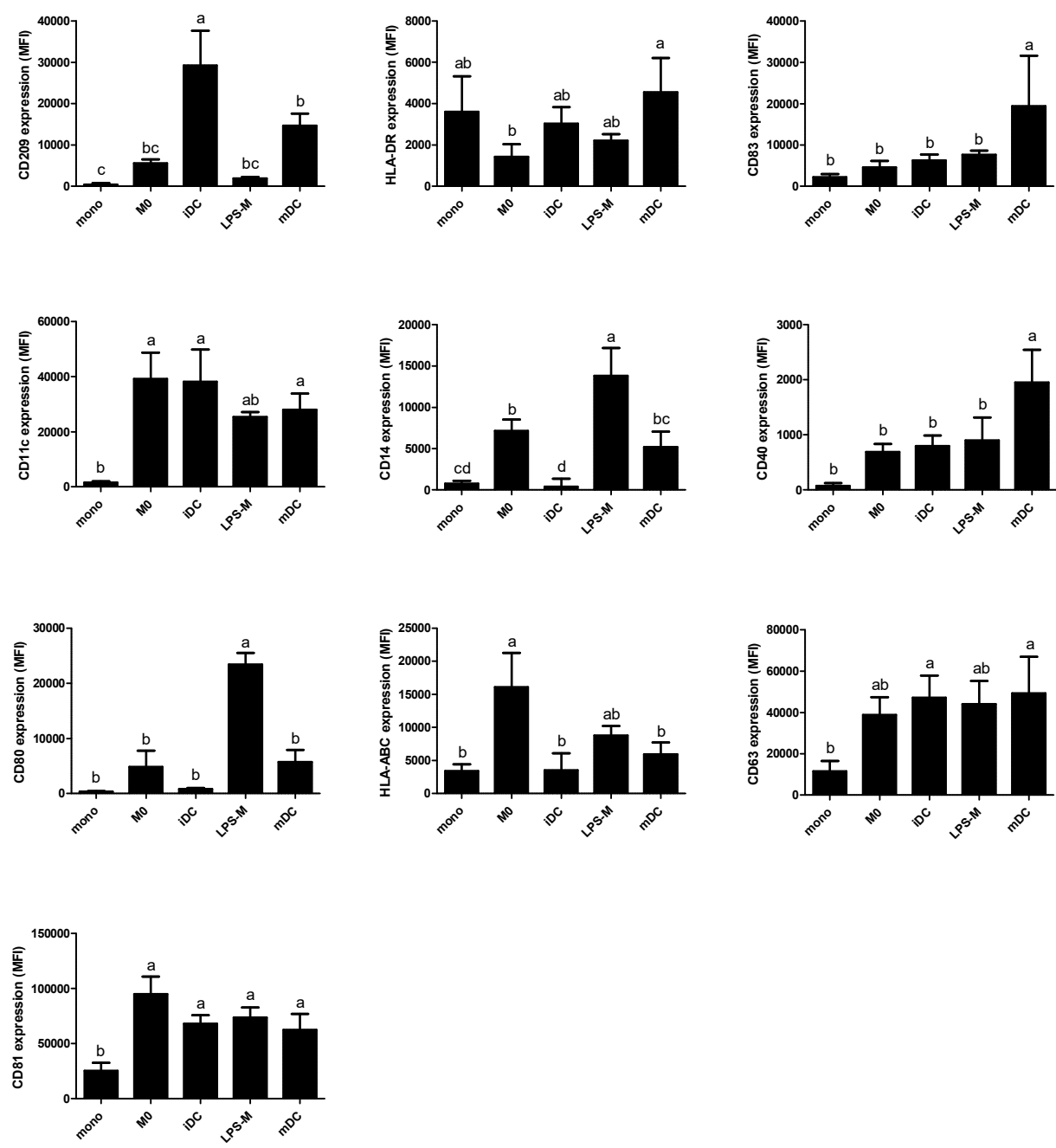

Figure 4.4 THP-1 derived MO and iDC and their LPS-stimulated forms LPS-M and mDC showed different surface marker profiles compared to monocytes. THP-1 monocytes were differentiated into macrophage-like and dendritic-like cells and stimulated with LPS. Expression of surface markers was checked using fluorescence-labelled antibodies and MFI was measured by flow cytometry. The results represent the mean MFI \pm SD of 5 independent experiments. The statistical differences were calculated with Fishers Least Significant Difference (LSD) test and bars with different letters represent significant differences $(p<0.05)$.

The secretion of cytokines also showed significant differences between MO and iDC, and their corresponding cell types after LPS stimulation (Figure 4.5). Monocytes did not produce any of the 
tested cytokines. There was no significant difference in the secretion levels of IL-6 and IL-10 (inflammation regulatory and anti-inflammatory cytokines) by MO and IDC, which were both nonsignificant compared to monocytes. However, the secretion levels of IL-8 and TNF- $\alpha$ (proinflammatory cytokines) in iDC were significantly higher than M0 (avg. 22.0 vs. 0.2 and $0.3 \mathrm{vs.} 0 \mathrm{ng} / \mathrm{mL}$ respectively). LPS stimulation led to higher cytokine production for both stimulated cell types compared to their corresponding non-stimulated cells for all tested cytokines. The significantly higher secretion levels of IL-8 and TNF $\alpha$ in iDC compared to MO were maintained in their LPS-stimulated cells with a general higher production level (avg. 75.8 vs. 57.1 and 3.9 vs. $2.2 \mathrm{ng} / \mathrm{mL}$ ). On the contrary, the production of IL-6 and IL-10 in LPS-M became significantly higher than in $\mathrm{mDC}$ (avg. 1.7 vs. 0.4 and $3.9 \mathrm{vs.} 0.5 \mathrm{ng} / \mathrm{mL}$ ). IL-12 production was not detected in any of the cell types or differentiation stages (data not shown).

A

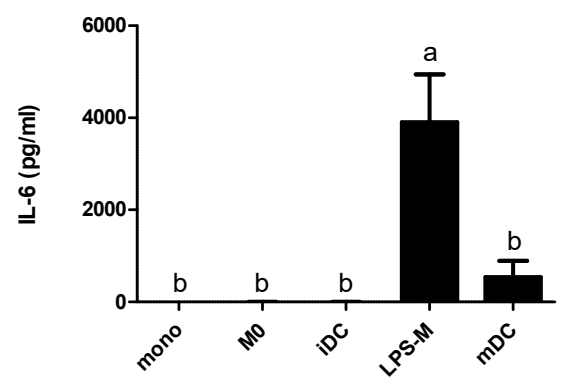

C

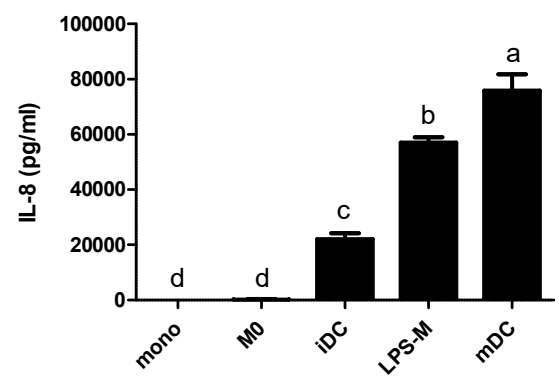

B

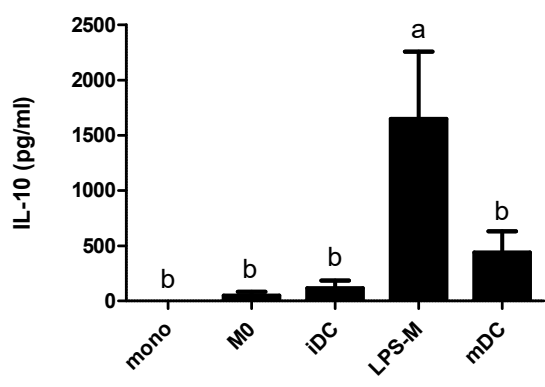

D

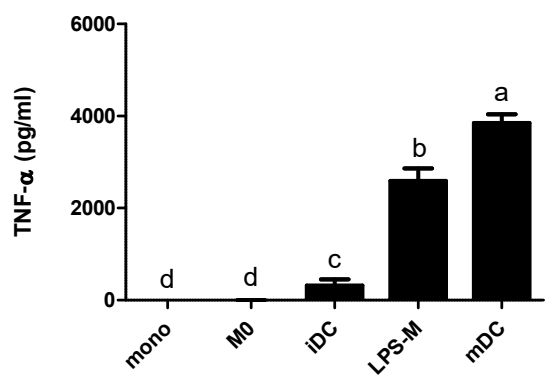

Figure 4.5 Cytokine production of monocytes (mono) and derived macrophages in the resting stage (MO) and immature dendritic cells (iDC), LPS-induced macrophages (LPS-M) and mature dendritic cells (mDC) differs. THP-1 monocytes were differentiated into macrophage-like and dendritic-like cells and stimulated with LPS. The supernatant of the cell cultures was collected, and the cytokines were measured using corresponding ELISA kits. The results represent mean values $\pm S D$ of 2-4 independent experimental measurements. The statistical differences were calculated with Fishers Least Significant Difference (LSD) test and bars with different letters represent significant differences $(p<0.05)$. 


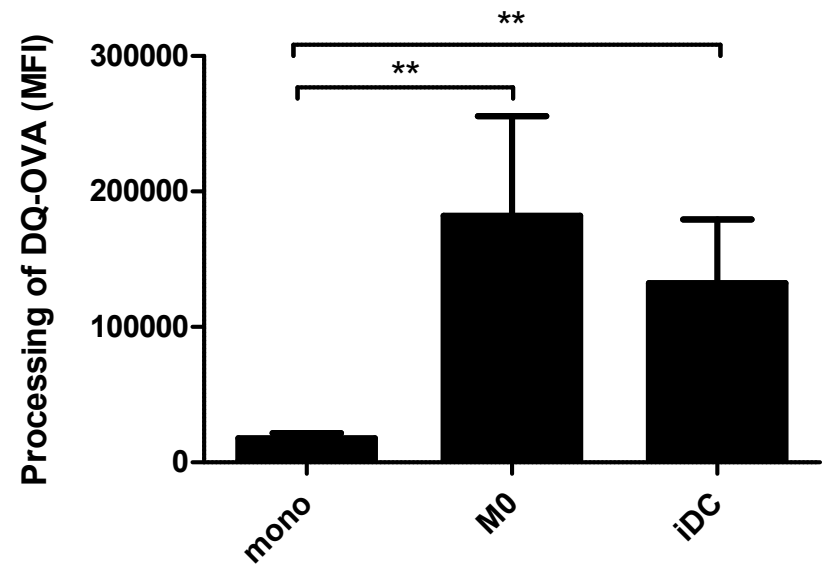

Figure 4.6 The antigen processing ability of macrophage-like cells at resting stage (MO) and immature dendritic-like cells (IDC) are significantly higher than that of THP-1 monocytes (mono). DQ-OVA shows florescence when it is taken up and processed by phagocytes. THP-1 monocytes were differentiated into macrophage-like and dendritic-like cells and incubated with DQ-OVA for 2 hours, after which the florescence signal was measured by flow cytometry. The results represent mean MFI $\pm S D$ of 5 independent experimental measurements. The statistical differences between any two data set were calculated with two-tailed unpaired T-test, ${ }^{* *} p<0.01$.

A key hallmark of APCs is the internalization and processing of antigens for subsequent presentation to adaptive immune cells. Using DQ-OVA, we tested the antigen uptake and processing capacity of MO and iDC. Compared to monocytes, both $\mathrm{MO}$ and iDC showed significantly higher antigen processing ability (Figure 4.6).

\section{Treatment of MO and iDC with processed BLG led to different gene transcription profile}

The THP-1 derived MO and IDC model was used further to study the impact of processed BLG samples on the transcription levels. The cells were exposed to native, wet-heated, high- and low-temperature dry-heated BLG in the presence glucose (BLG, W-glu-BLG, H-glu-BLG and L-glu-BLG), after which the gene transcription levels were analysed using microarray and compared to non-treated M0 and iDC.

In general, there were more upregulated genes than downregulated in either cell type treated by either sample type. In MO treated with BLG and L-glu-BLG, the highest total number of significantly differentially transcribed genes (fold change absolute value $>2, q<0.05$ ) was found (246 and 172 respectively; Figure $4.7 \mathrm{~A}$ ). Figure $4.7 \mathrm{C}$ showed that most of the significantly differentially transcribed genes in $\mathrm{MO}$ incubated with L-glu-BLG were also transcribed in $\mathrm{MO}$ incubated with BLG. In MO treated 
with H-glu-BLG or W-glu-BLG and iDC treated with any sample, the number of significantly differentially transcribed genes was below 20 (Figure 4.7A and 4.7B), for which details can be found in Table 4.S4 and S5.

A

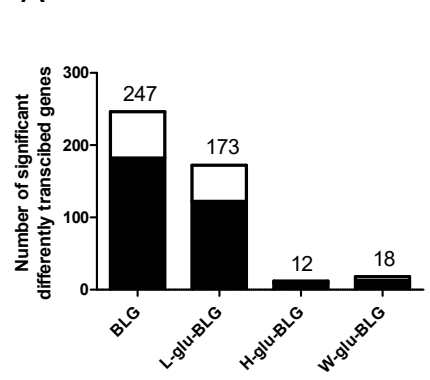

C
B

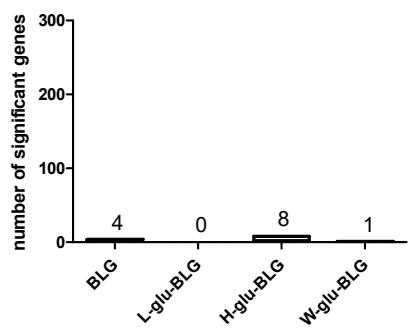

up-regulated
down-regulated
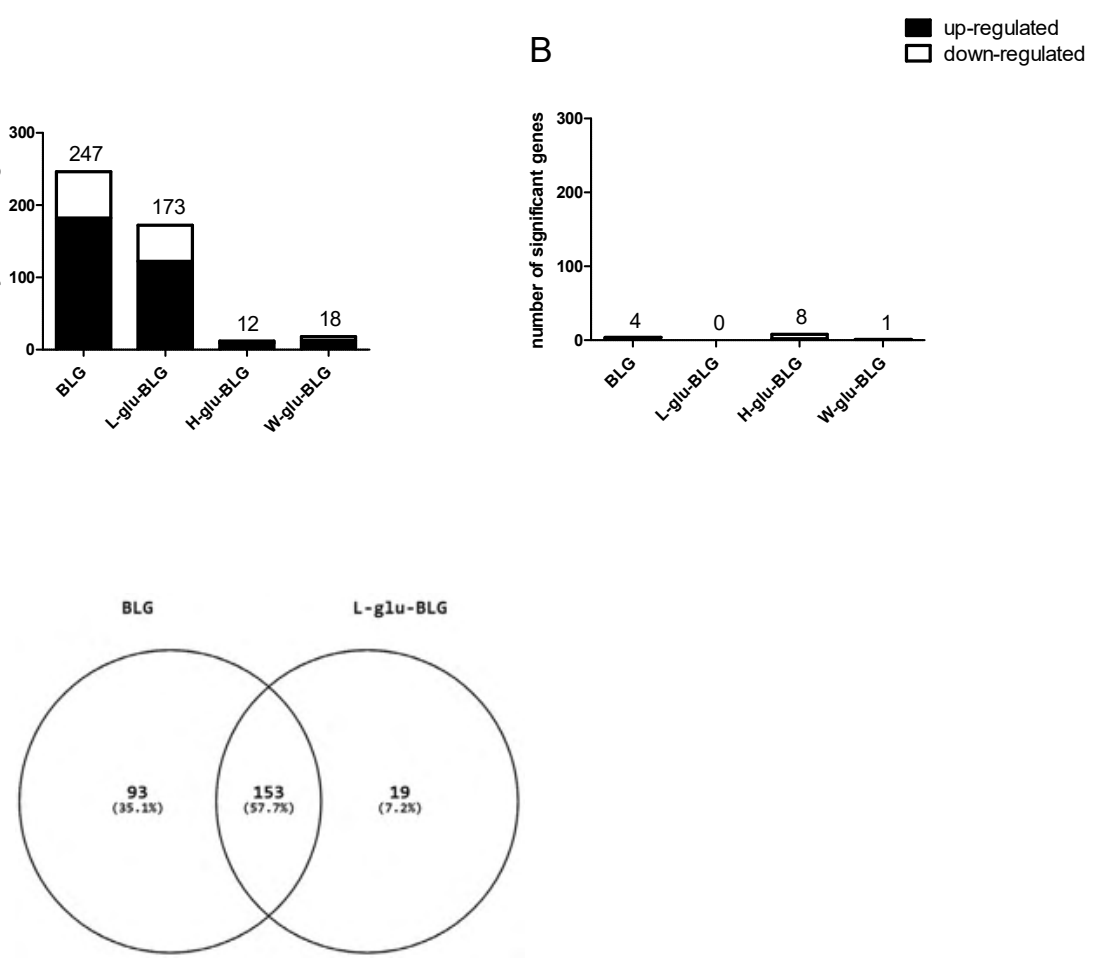

Figure 4.7 THP-1 derived MO and iDC responded with different gene transcription profiles to native or processed BLG. THP-1 monocytes were differentiated into macrophage-like and dendritic-like cells and incubated with native and processed BLG samples for 6 hours. The gene transcription level of cells was measured by microarray based on isolated RNA and expressed as fold change compared to the gene transcription level of medium control cells. The bar chart indicated the number of significantly (with fold change absolute value $>2$ and $q$ value $<0.05$ ) up or down regulated genes for different BLG sample treated $M O(A)$ and $I D C(B)$. Venn diagram (C) showed the numbers of significantly transcribed genes which are shared or exclusive for MO incubated with BLG or L-glu-BLG.

\section{Innate immunity related pathways and upstream regulators were significantly associated or activated in for MO treated with native or low-temperature dry-heated BLG}

The gene transcription profiles of MO treated with native or low-temperature dry-heated BLG were integrated and interpreted further by IPA to obtain a view towards potentially related immune 
responses. Analysis of the canonical pathways (Figure 4.8) showed that both BLG and L-glu-BLG induced immune related pathways in $\mathrm{MO}$ after incubation. Inflammation induction-related pathways were significantly associated with M0 treated with BLG and L-glu-BLG, including Th1 Pathway, NF-KB Signaling, TNFR1 Signaling, TWEAK Signaling, Activation of IRF by Cytosolic Pattern Recognition Receptors, TNFR1 Signaling, Toll-like Receptor Signaling, CD40 Signaling, HMGB1 Signaling, TREM1 Signaling. Additionally, pathways like IL-23 Signaling Pathway (Th17 maintenance and expansion), Communication between Innate and Adaptive Immune Cells, Agranulocyte Adhesion and Diapedesis, Granulocyte Adhesion and Diapedesis and IL-15 Production (regulatory of natural killer cells and T cells) which are related to adaptive immunity signalling were also significantly associated with MO treated with BLG and L-glu-BLG. Compared to MO incubated with BLG, MO incubated with L-glu-BLG did not show significant association with Th17 Activation Pathway and IL-12 Signaling and Production in Macrophages. On the contrary, several B cell related pathways and Th2 pathway were only significant associated in $\mathrm{MO}$ incubated with L-glu-BLG.

\section{Canonical pathways}

BLG L-glu-BLG

IL-23 Signaling Pathway

Dendritic Cell Maturation

IL-17A Signaling in Gastric Cells

Role of Hypercytokinemia/hyperchemokinemia in the Pathogenesis of Influenza Hepatic Fibrosis / Hepatic Stellate Cell Activation

Role of IL-17A in Arthritis

Altered T Cell and B Cell Signaling in Rheumatoid Arthritis

Communication between Innate and Adaptive Immune Cells

Th1 Pathway

Tryptophan Degradation to 2-amino-3-carboxymuconate Semialdehyde

L-serine Degradation

NF-kB Signaling

Airway Pathology in Chronic Obstructive Pulmonary Disease

Agranulocyte Adhesion and Diapedesis

Granulocyte Adhesion and Diapedesis

Hepatic Fibrosis Signaling Pathway

4-1BB Signaling in T Lymphocytes

TWEAK Signaling

Induction of Apoptosis by HIV1

Activation of IRF by Cytosolic Pattern Recognition Receptors

Lymphotoxin $\beta$ Receptor Signaling

TNFR1 Signaling

Role of IL-17F in Allergic Inflammatory Airway Diseases

IL-15 Production

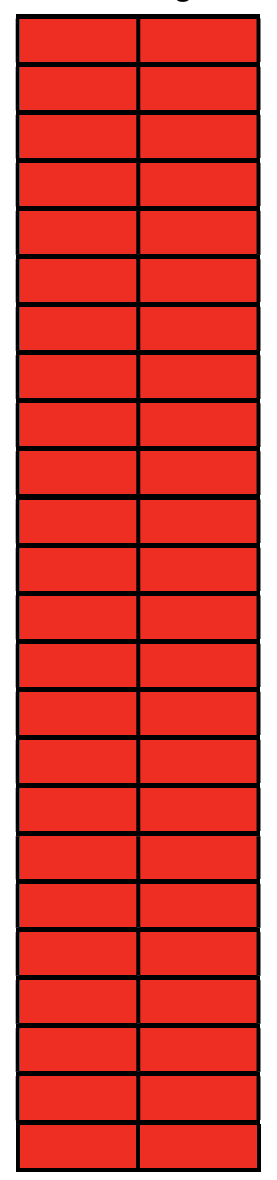


Crosstalk between Dendritic Cells and Natural Killer Cells

FAT10 Cancer Signaling Pathway

TNFR2 Signaling

Toll-like Receptor Signaling

CD40 Signaling

Neuroinflammation Signaling Pathway

HMGB1 Signaling

TREM1 Signaling

Hepatic Cholestasis

Th17 Activation Pathway

Pathogenesis of Multiple Sclerosis

IL-12 Signaling and Production in Macrophages

Relaxin Signaling

G-Protein Coupled Receptor Signaling

Atherosclerosis Signaling

iCOS-iCOSL Signaling in T Helper Cells

IL-6 Signaling

Gas Signaling

April Mediated Signaling

B Cell Activating Factor Signaling

Role of PKR in Interferon Induction and Antiviral Response CD27 Signaling in Lymphocytes

Systemic Lupus Erythematosus In B Cell Signaling Pathway

B Cell Development

Th1 and Th2 Activation Pathway

Th2 Pathway

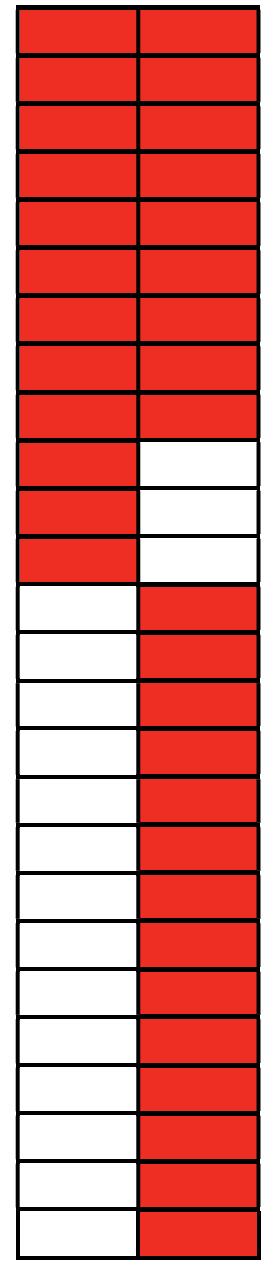

Figure 4.8 Significantly associated canonical pathways in MO incubated with BLG and L-glu-BLG were mostly related with immune response. THP-1 monocytes were differentiated macrophage-like (MO) were incubated with native or low-temperature dry-heated BLG in the presence of glucose, and their gene transcription levels were imported into IPA for canonical pathway analysis. Canonical pathways with a $p$ value $<0.001$ were labelled in red and listed from top to bottom followed the $p$ value difference between $M O$ and iDC from large to small for categories of $p<0.001$ in both MO and iDC, only MO and only iDC separately.

As shown in Table 4.S6, in MO treated with native BLG, innate immune related regulators ECSIT (intermediate In Toll Pathway), IFNG (IFN $\gamma)$, TLR4, IL27, TLR2, TNF (TNF- $\alpha$ ) and IL-1 $\beta$ were significantly activated while LY6E which has immunosuppressive effects [34] was inhibited. There was no clear 
difference in the activated or inhibited upstream regulators in $\mathrm{MO}$ incubated with L-glu-BLG compared to $B L G$, but the activation levels (based on z-scores) were weaker in the former condition.

Cytokine transcription and secretion level differed for native and processed BLG treated MO and iDC

Cytokines that regulate cell signalling and immune response were further investigated for estimating the influence of processing of BLG on innate immune cell responses. From the formerly described transcription data (microarray), cytokine genes with a significantly differentially transcription level in MO following incubation with any BLG samples are presented in Table 4.1 (no significantly differentially transcribed cytokine gene for iDC). In fact, differential transcription was found only in MO incubated with BLG or L-glu-BLG with transcription levels being slightly higher in BLG stimulated MO. Significantly higher transcription levels were found for IL23A, IL32, CCL1, CCL4, CCL5, CCL19, CCL22, CXCL3, CXCL8, CXCL10 and CXCL11 in BLG treated M0 compared to medium-treated M0.

Table 4.1 Transcription fold change of cytokine genes in MO incubated with BLG samples

\begin{tabular}{lcccc}
\hline Cytokine & BLG & L-glu-BLG & H-glu-BLG & W-glu-BLG \\
\hline IL23A & $2.3^{*}$ & 1.6 & 1.2 & 1.0 \\
IL32 & $3.4^{*}$ & $3.8^{*}$ & 1.8 & 2.1 \\
CCL1 & $4.9^{*}$ & $2.6^{*}$ & 1.7 & 1.4 \\
CCL4 & $4.5^{*}$ & 3.3 & 1.3 & 1.6 \\
CCL5 & $2.2^{*}$ & $1.8^{*}$ & 1.1 & 1.2 \\
CCL19 & $4.9^{*}$ & $4.3^{*}$ & 1.3 & 1.5 \\
CCL22 & $3.7^{*}$ & $2.7^{*}$ & 1.6 & 1.6 \\
CXCL3 & $3.5^{*}$ & 3.3 & -1.2 & 1.1 \\
CXCL8 & $14.0^{*}$ & 9.0 & 2.2 & 3.5 \\
CXCL10 & $3.8^{*}$ & $5.7^{*}$ & 1.4 & 2.1 \\
CXCL11 & $2.1^{*}$ & 1.8 & 1.3 & 1.2 \\
\hline
\end{tabular}

Note: The values indicate fold changes of the transcription of THP-1 derived MO and IDC exposed to different BLG samples compared to their control (exposed to culture medium) of $N=3$ experiments, ${ }^{*} q$ $<0.05$.

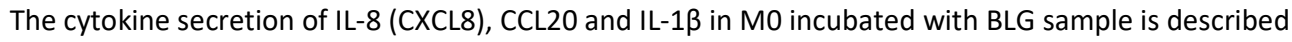
in the next chapter, in which we showed a significantly higher secretion of all cytokines by MO incubated with BLG and L-glu-BLG compared to other BLG samples. In iDC, BLG and L-glu-BLG similarly induced a significantly increased secretion of IL-8 and IL-1 $\beta$, but not CCL20 (Figure 4.9). 
A

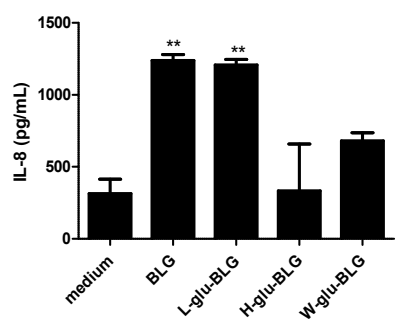

B

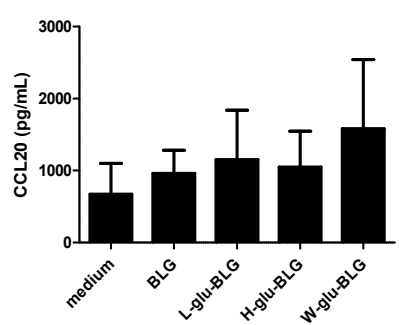

C

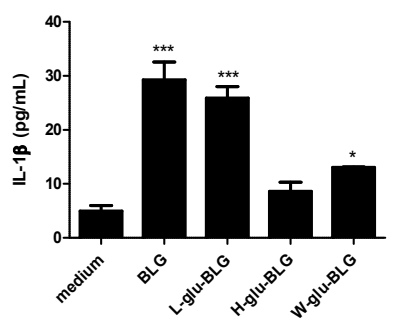

Figure 4.9 Cytokine response of iDC upon exposure to native or heat-treated BLG varies. BLG was untreated or heated (W, $H$ or $L$ ) in the presence of glucose and incubated with iDC. The cytokine secretion of IL-8 (A), CCL2O (B) and IL-1B (C) was analysed in the supernatant with ELISA. The results represent mean values $\pm S D$ of 3 independent experimental measurements. The statistical differences were calculated with Dunnett's Test compared to medium control: ${ }^{*} p<0.05 ;{ }^{* *} p<0.01 ;{ }^{* *} p<0.001$.

\section{Discussion}

The differentiation of THP-1 monocytes into macrophage-like cells (MO) by PMA is a common method, whereas studies about the differentiation into dendritic-like cells are relatively limited [35]. Based on the protocol of Katayama et al. [20], we generated THP-1 derived dendritic-like cells (iDC) using PMA and IL-4. The morphology, transcription profile, expression and secretion levels of surface markers and cytokines, and antigen processing capability of THP-1 derived MO and IDC were determined, and these were compared to their progenitor monocytes. Although PMA was used to derive both cell types, the lower concentration of PMA and the addition of IL-4 induced dendritic-like cells as a clearly different cell type from macrophages and monocytes.

The difference of MO and IDC from their progenitor THP-1 monocytes is clear from their morphology (Figure 4.1), as well as the vast number of significantly transcribed genes compared to monocytes (Figure 4.2). There were in total 5254 genes significantly differentially transcribed in MO and iDC compared to monocytes, of which about half were shared by both cell types. From the canonical pathway analysis in Figure 4.3, both MO and iDC showed significant association with the cell differentiation and immune response related pathways, indicating their differentiated cell identities and essential role in immune responses. Their antigen processing capabilities were illustrated by the significantly higher MFI signals due to the processing of DQ-OVA compared to monocytes, as shown in Figure 4.6. 
The difference between iDC and MO was also obvious from their morphology, with a typical circular shape and the presence of dendrites for iDC (Figure 4.1). When looking at the significantly differentially transcribed genes compared to monocytes, the correlation between the fold change of MO and iDC is weak (Figure 4.2A). The fact that iDC had a higher number of significantly differentially transcribed genes than M0 might be caused by stronger activation of upstream regulators (TNF- $\alpha$, TLR4, TREM1 and TSLP) and secretion of IL-8 and TNF- $\alpha$ compared to M0 (Table 4.S3 and Figure 4.5). TNF- $\alpha$ and TLR4 play essential roles in activating $T$ cells $[36,37]$. This coincided with the significantly associated pathways Leukocyte Extravasation Signaling and IL-10 Signaling (Figure 4.3). CD209, which was significantly stronger expressed in IDC than in THP-1 monocytes and MO, was also reported to be involved in dendritic cell-T cell interaction [38]. These observations indicate the additional $T$ cell signalling function of iDC next to their role in phagocytosis and other immune regulatory functions as APCs [39]. On the other hand, MO showed exclusive and significant associations with uptake and proinflammatory related pathways (Figure 4.3), which is in line with the more prominent role of $\mathrm{MO}$ in inducing phagocytosis and inflammation compared to iDC. Expression of CD14 was significantly higher in $\mathrm{MO}$, which is reported to be a pattern recognition receptor for LPS and related to the innate immune response of macrophages [40]. LPS-stimulated M0 secreted significantly higher amounts of IL6 and IL10 (Figure 4.5), which is in line with other studies [41, 42]. The transcription levels of the surface markers (Table 4.S7) corroborated the outcomes of the surface marker expression for both $\mathrm{MO}$ and iDC, and even included more surface marker genes. For instance, CD3G, CD22, CD36, CD109, CD163, CD180 showed clearly higher gene transcription levels for M0 than for iDC, while CD1B, CD1C, CD4, CD86, CD226, CD274, CD300LB were more transcribed by iDC than by M0. These surface markers may have potential value in distinguishing MO and IDC for future studies. Overall, our THP-1 derived M0 and IDC could be distinguished from each other and showed clearly differing properties. Moreover, they successfully represented the primary derived macrophages and dendritic cells by showing the strengths in antigen uptake, processing and inflammatory response by $\mathrm{MO}$ and the function of $\mathrm{T}$ cell stimulation and signalling between the innate and adaptive immune system by iDC.

Although iDC had more significantly differentially transcribed genes and higher transcription levels compared to monocytes than MO, its response to BLG samples was much weaker than $\mathrm{MO}$ in both transcription and cytokine secretion levels (Figure 4.7 and 4.9). BLG was reported to have limited immunomodulatory effects when incubated with murine DC [43], whereas its immune effect on macrophages has not been studied yet besides in our uptake study (Chapter 3). As shown in Table 4.1 and Figure 4.9, MO incubated with native and low-temperature dry-heated BLG induced the transcription and secretion of cytokine genes to higher levels compared to wet-heated and hightemperature dry-heated BLG. The lower cytokine secretion levels of wet-heated and high-temperature 
dry-heated BLG are probably due to their more severe structural changes after processing, as indicated previously (Chapter 3).

The inflammatory pathways and activation of innate immunity related upstream regulators in $\mathrm{M} 0$ that were significantly associated with incubation with BLG and L-glu-BLG (Figure 4.8 and Table 4.S6) appear to indicate a pro-inflammatory effect of BLG. There were also quite a number of adaptive immunity and $\mathrm{T}$ cell signalling pathways activated, which is not surprising as other studies also demonstrated that macrophages have the ability to regulate adaptive immune responses and even directly contact $T$ cells $[44,45]$. The pro-inflammatory effect of BLG and L-glu-BLG could possibly be linked with the significant association of Thelper 1 (Th1) Pathways [46]. In addition to the Th1 response, the response of MO to incubation with the BLG sample also seemed to impact Th17 cells, as suggested by the significant association with IL-23, Th17 and several IL-17 related pathways. There was no clear difference in the activation of upstream regulators by MO incubated with BLG or L-glu-BLG, and they also shared most of the significantly associated pathways. Glycation in L-glu-BLG, as previously reported (Chapter 3 ), might still influence the immunogenicity of BLG to some extent. As indicated in Figure 4.9, IL-6 Signaling and Th2 Pathway is only significantly associated with MO incubated with Lglu-BLG. This observation is in line with the finding of the stronger enhancing effect of ovalbumin glycated at $50^{\circ} \mathrm{C}$ for 6 weeks than native ovalbumin towards IL- 6 secretion and Th2 differentiation [47]. This study, where human primary monocyte-derived dendritic cells were used and Th2 cytokine (IL-4 and IL-5) secretion levels were measured based on $\mathrm{CD}^{+} \mathrm{T}$ cells co-cultures of dendritic cells, showed that native ovalbumin was also associated with Th1 induction.

\section{Conclusion}

Endocytic uptake is the crucial and fundamental step in inducing further primary responses by APCS [48]. As indicated by our results, MO incubated with BLG samples showed stronger transcriptional responses than iDC. Wet-heated BLG, which has the severest physicochemical modification and the highest uptake by APCs among all tested samples (Chapter 3 and 5), did not cause the strongest transcriptional response by innate immune cells. There is thus no clear relationship between the uptake efficiency in $\mathrm{MO}$ and the transcription-inducing effects of BLG. Glycation, as occurring at lowtemperature dry-heated conditions, might change the immunogenicity of BLG towards a Th2 differentiation enhancing direction which is related to chronic inflammatory disease and allergy [49]. In conclusion, dendritic-like cells were generated successfully from THP-1 monocytes, which clearly differed from both their progenitor and MO. In addition, the uptake of protein by APCs was shown to not be linked with gene transcription and cytokine production. It should be noted that this work is based on in vitro experiments, the result of which still need to be further verified in vivo, as functional 
responses of immune cells, rather than uptake or gene transcription alone, are required to identify immunogenicity of proteins before and after processing-induced changes. 


\section{References}

1. Mills, E.N.C., A.S. Tatham, and B. Caballero, ALLERGENS, in Encyclopedia of Food Sciences and Nutrition (Second Edition). 2003, Academic Press: Oxford. p. 143-150.

2. Rahaman, T., T. Vasiljevic, and L. Ramchandran, Effect of processing on conformational changes of food proteins related to allergenicity. Trends in Food Science \& Technology, 2016. 49: p. 24-34.

3. Tsabouri, S., K. Douros, and K.N. Priftis, Cow's milk allergenicity. Endocrine, Metabolic and Immune Disorders - Drug Targets, 2014. 14(1): p. 16-26.

4. Morr, C.V., Functionality of Heated Milk Proteins in Dairy and Related Foods. Journal of Dairy Science, 1985. 68(10): p. 2773-2781.

5. Villa, C., et al., Bovine Milk Allergens: A Comprehensive Review. Comprehensive Reviews in Food Science and Food Safety, 2018. 17(1): p. 137-164.

6. Rytkönen, J., et al., Effect of heat denaturation on beta-lactoglobulin-induced gastrointestinal sensitization in rats: Denatured BLG induces a more intensive local immunologic response than native BLG. Pediatric Allergy and Immunology, 2002. 13(4): p. 269-277.

7. Wal, J.-M., Cow's milk allergens. Allergy, 1998. 53(11): p. 1013-1022.

8. $\mathrm{Bu}, \mathrm{G} . \mathrm{H}$. , et al., Effect of heat treatment on the antigenicity of bovine -lactalbumin and lactoglobulin in whey protein isolate. Food and Agricultural Immunology, 2009. 20(3): p. 195206.

9. Zenker, H.E., et al., Differential Effects of Dry vs. Wet Heating of beta-Lactoglobulin on Formation of sRAGE Binding Ligands and slgE Epitope Recognition. Nutrients, 2019. 11(6).

10. Schuijs, M.J., H. Hammad, and B.N. Lambrecht, Professional and 'Amateur' Antigen-Presenting Cells In Type 2 Immunity. Trends in Immunology, 2019. 40(1): p. 22-34.

11. Savina, A. and S. Amigorena, Phagocytosis and antigen presentation in dendritic cells. Immunological Reviews, 2007. 219: p. 143-156.

12. Chanput, W., J.J. Mes, and H.J. Wichers, THP-1 cell line: An in vitro cell model for immune modulation approach. International Immunopharmacology, 2014. 23(1): p. 37-45.

13. Daigneault, M., et al., The Identification of Markers of Macrophage Differentiation in PMAStimulated THP-1 Cells and Monocyte-Derived Macrophages. PLOS ONE, 2010. 5(1): p. e8668.

14. Riddy, D.M., et al., Comparative genotypic and phenotypic analysis of human peripheral blood monocytes and surrogate monocyte-like cell lines commonly used in metabolic disease research. PLOS ONE, 2018. 13(5): p. e0197177.

15. Kohro, T., et al., A comparison of differences in the gene expression profiles of phorbol 12myristate 13-acetate differentiated THP-1 cells and human monocyte-derived macrophage. Journal of atherosclerosis and thrombosis, 2004. 11(2): p. 88-97.

16. Auwerx, J., The human leukemia cell line, THP-1: a multifacetted model for the study of monocyte-macrophage differentiation. Experientia, 1991. 47(1): p. 22-31.

17. Katayama, S., et al., Apple polyphenols suppress antigen presentation of ovalbumin by THP-1derived dendritic cells. Food Chemistry, 2013. 138(2): p. 757-761.

18. Ferenbach, D. and J. Hughes, Macrophages and dendritic cells: what is the difference? Kidney International, 2008. 74(1): p. 5-7.

19. Chanput, W., et al., Characterization of polarized THP-1 macrophages and polarizing ability of LPS and food compounds. Food Funct, 2013. 4(2): p. 266-76.

20. Katayama, S., et al., Apple polyphenols suppress antigen presentation of ovalbumin by THP-1derived dendritic cells. Food Chemistry, 2013. 138(2-3): p. 757-761.

21. de Jongh, H.H.J., T. Groneveld, and J. de Groot, Mild isolation procedure discloses new protein structural properties of beta-lactoglobulin. Journal of Dairy Science, 2001. 84(3): p. 562-571.

22. Tang, Y.F., et al., Macrophages treated with non-digestible polysaccharides reveal a transcriptionally unique phenotype. Journal of Functional Foods, 2017. 36: p. 280-289. 
23. Lin, K., et al., MADMAX - Management and analysis database for multiple omics experiments. J Integr Bioinform, 2011. 8(2): p. 160.

24. Izuhara, K., et al., Squamous Cell Carcinoma Antigen 2 (SCCA2, SERPINB4): An Emerging Biomarker for Skin Inflammatory Diseases. International Journal of Molecular Sciences, 2018. 19(4).

25. Okawa, T., et al., Serum levels of squamous cell carcinoma antigens 1 and 2 reflect disease severity and clinical type of atopic dermatitis in adult patients. Allergology International, 2018. 67(1): p. 124-130.

26. Zamani, F., et al., Induction of CD14 Expression and Differentiation to Monocytes or Mature Macrophages in Promyelocytic Cell Lines: New Approach. Adv Pharm Bull, 2013. 3(2): p. 32932.

27. Laupeze, B., et al., Differential expression of major histocompatibility complex class la, Ib and II molecules on monocytes and monocyte-derived dendritic and macrophagic cells. Human Immunology, 1999. 60(7): p. 591-597.

28. Cheong, C., et al., Microbial Stimulation Fully Differentiates Monocytes to DC-SIGN/CD209(+) Dendritic Cells for Immune T Cell Areas. Cell, 2010. 143(3): p. 416-429.

29. Hume, D.A., Macrophages as APC and the Dendritic Cell Myth. Journal of Immunology, 2008. 181(9): p. 5829-5835.

30. Peng, W.M., et al., Tetraspanins CD9 and CD81 are molecular partners of trimeric FcvarepsilonRI on human antigen-presenting cells. Allergy, 2011. 66(5): p. 605-11.

31. Mantegazza, A.R., et al., CD63 tetraspanin slows down cell migration and translocates to the endosomal-Iysosomal-MIICs route after extracellular stimuli in human immature dendritic cells. Blood, 2004. 104(4): p. 1183-1190.

32. Nagai, T., Difference between Immature Dendritic Cells (imDCs) and Mature Dendritic Cells ( $m D C s)$ Derived from Human Monocytes. Journal of Immunology, 2017. 198(1).

33. Raggi, F., et al., Regulation of Human Macrophage M1-M2 Polarization Balance by Hypoxia and the Triggering Receptor Expressed on Myeloid Cells-1. Front Immunol, 2017. 8: p. 1097.

34. Xu, X., et al., IFN-Stimulated Gene LYGE in Monocytes Regulates the CD14/TLR4 Pathway but Inadequately Restrains the Hyperactivation of Monocytes during Chronic HIV-1 Infection. Journal of Immunology, 2014. 193(8): p. 4125-4136.

35. Starr, T., et al., The phorbol 12-myristate-13-acetate differentiation protocol is critical to the interaction of THP-1 macrophages with Salmonella Typhimurium. Plos One, 2018. 13(3).

36. Bauman, S.K., G.B. Huffnagle, and J.W. Murphy, Effects of tumor necrosis factor alpha on dendritic cell accumulation in lymph nodes draining the immunization site and the impact on the anticryptococcal cell-mediated immune response. Infection and Immunity, 2003. 71(1): p. 68-74.

37. Fang, H.L., et al., TLR4 is essential for dendritic cell activation and anti-tumor T-cell response enhancement by DAMPs released from chemically stressed cancer cells. Cellular \& Molecular Immunology, 2014. 11(2): p. 150-159.

38. Geijtenbeek, T.B.H., et al., Identification of DC-SIGN, a novel dendritic cell-specific ICAM-3 receptor that supports primary immune responses. Cell, 2000. 100(5): p. 575-585.

39. Guilliams, M., et al., Dendritic cells, monocytes and macrophages: a unified nomenclature based on ontogeny. Nature Reviews Immunology, 2014. 14(8): p. 571-578.

40. Wright, S.D., et al., Cd14, a Receptor for Complexes of Lipopolysaccharide (Lps) and Lps BindingProtein. Science, 1990. 249(4975): p. 1431-1433.

41. Rego, D., et al., IL-6 Production Is Positively Regulated by Two Distinct Src Homology Domain 2-Containing Tyrosine Phosphatase-1 (SHP-1)-Dependent CCAAT/Enhancer-Binding Protein beta and NF-kappa B Pathways and an SHP-1-Independent NF-kappa B Pathway in Lipopolysaccharide-Stimulated Bone Marrow-Derived Macrophages. Journal of Immunology, 2011. 186(9): p. 5443-5456. 
42. Iyer, S.S., A.A. Ghaffari, and G. Cheng, Lipopolysaccharide-Mediated IL-10 Transcriptional Regulation Requires Sequential Induction of Type I IFNs and IL-27 in Macrophages. Journal of Immunology, 2010. 185(11): p. 6599-6607.

43. Brix, S., et al., Immunostimulatory potential of beta-lactoglobulin preparations: Effects caused by endotoxin contamination. Journal of Allergy and Clinical Immunology, 2003. 112(6): p. 12161222.

44. Hilhorst, M., et al., $T$ cell-macrophage interactions and granuloma formation in vasculitis. Frontiers in Immunology, 2014. 5: p. 1-14.

45. Roberts, C.A., A.K. Dickinson, and L.S. Taams, The Interplay Between Monocytes/Macrophages and CD4(+) T Cell Subsets in Rheumatoid Arthritis. Frontiers in Immunology, 2015. 6: p. 1-19.

46. Berger, A., Science commentary: Th1 and Th2 responses: what are they? British Medical Journal, 2000. 321(7258): p. 424-424.

47. Hilmenyuk, T., et al., Effects of glycation of the model food allergen ovalbumin on antigen uptake and presentation by human dendritic cells. Immunology, 2010. 129(3): p. 437-445.

48. Frei, P.C., Benacerr.B, and Thorbeck.Gj, Phagocytosis of Antigen a Crucial Step in Induction of Primary Response. Proceedings of the National Academy of Sciences of the United States of America, 1965. 53(1): p. 20-\&.

49. Walker, J.A. and A.N.J. McKenzie, TH2 cell development and function. Nat Rev Immunol, 2018. 18(2): p. 121-133. 


\section{Supplementary data}

Table 4.S1 Top 25 genes with the largest difference in transcription between MO and iDC- higher in Mo

\begin{tabular}{|c|c|c|c|}
\hline $\begin{array}{l}\text { Gene } \\
\text { name }\end{array}$ & Mo & iDC & Gene Ontology Description \\
\hline CYBB & $15.1^{*}$ & -1.6 & $\begin{array}{l}\text { inflammatory response, antigen processing and presentation of } \\
\text { peptide antigen via MHC class I }\end{array}$ \\
\hline IL7R & $10.3^{*}$ & -2.1 & regulation of DNA recombination, signal transduction \\
\hline EDNRA & $19.3^{*}$ & 1.2 & respiratory gaseous exchange, glucose transport \\
\hline PRR5L & 13.5 & -1.1 & TORC2 signaling, negative regulation of protein phosphorylation \\
\hline PLK2 & $5.4^{*}$ & $-2.6^{*}$ & mitotic cell cycle checkpoint, Ras protein signal transduction \\
\hline S1PR1 & $18.3^{*}$ & 1.3 & $\begin{array}{l}\text { blood vessel maturation, cardiac muscle tissue growth involved in } \\
\text { heart morphogenesis }\end{array}$ \\
\hline CHRM3 & $17.9 *$ & 1.3 & $\begin{array}{l}\text { G-protein coupled acetylcholine receptor signaling pathway, signal } \\
\text { transduction }\end{array}$ \\
\hline C3orf80 & $2.4^{*}$ & $-5.2^{*}$ & NA \\
\hline DYSF & $66.4 *$ & $5.9 *$ & plasma membrane repair, vesicle fusion \\
\hline SLC9A9 & $21.1^{*}$ & 1.9 & ion transport, transmembrane transport \\
\hline CD180 & $9.7^{*}$ & -1.1 & $\begin{array}{l}\text { positive regulation of lipopolysaccharide-mediated signaling } \\
\text { pathway, B cell proliferation involved in immune response }\end{array}$ \\
\hline UST & $17.4^{*}$ & 1.7 & carbohydrate metabolic process, protein sulfation \\
\hline ANTXR1 & $3.3^{*}$ & $-3.1 *$ & signal transduction, actin cytoskeleton reorganization \\
\hline GALNT16 & $11.2^{*}$ & 1.1 & protein glycosylation \\
\hline C3AR1 & $11.6^{*}$ & 1.2 & $\begin{array}{l}\text { metabolic process, complement receptor mediated signaling } \\
\text { pathway }\end{array}$ \\
\hline HEY2 & $14.2 *$ & 1.5 & vasculogenesis, muscular septum morphogenesis \\
\hline SERPINI1 & $12.9^{*}$ & $1.4^{*}$ & $\begin{array}{l}\text { central nervous system development, peripheral nervous system } \\
\text { development }\end{array}$ \\
\hline NA & $11.0^{*}$ & 1.2 & NA \\
\hline WIPF3 & $12.6^{*}$ & 1.4 & $\begin{array}{l}\text { Fc-gamma receptor signaling pathway involved in phagocytosis, } \\
\text { innate immune response }\end{array}$ \\
\hline PPFIA2 & $7.3^{*}$ & -1.2 & signal transduction, cell-matrix adhesion \\
\hline KCNMA1 & $19.2^{*}$ & $2.2^{*}$ & blood coagulation, synaptic transmission \\
\hline MMP2 & $3.6^{*}$ & $-2.3^{*}$ & extracellular matrix disassembly, extracellular matrix organization \\
\hline TLR7 & $8.7^{*}$ & 1.0 & $\begin{array}{l}\text { toll-like receptor signaling pathway, toll-like receptor } 9 \text { signaling } \\
\text { pathway }\end{array}$ \\
\hline ZC3H12D & $2.8^{*}$ & $-3.0 *$ & negative regulation of cell growth, negative regulation of G1 \\
\hline CHODL & $14.3^{*}$ & 1.8 & $\begin{array}{l}\text { inflammatory response, antigen processing and presentation of } \\
\text { peptide antigen via } \mathrm{MHC} \text { class I }\end{array}$ \\
\hline
\end{tabular}


Table 4.S2 Top 25 genes with the largest difference in transcription between MO and iDC- higher in iDC

\begin{tabular}{|c|c|c|c|}
\hline Gene name & MO & iDC & Gene Ontology Description \\
\hline SERPINB4 & $-3.3^{*}$ & $515.0^{*}$ & negative regulation of peptidase activity, regulation of proteolysis \\
\hline SERPINB3 & -1.1 & $135.2^{*}$ & response to virus, negative regulation of peptidase activity \\
\hline AQP9 & $2.8^{*}$ & $243.9 *$ & metabolic process, transport \\
\hline CCL22 & -1.5 & $51.5^{*}$ & cell-cell signaling, response to virus \\
\hline SERPINB12 & 1.3 & $70.7^{*}$ & $\begin{array}{l}\text { negative regulation of protein catabolic process, negative } \\
\text { regulation of endopeptidase activity }\end{array}$ \\
\hline SERPINB13 & -1.2 & $45.9 *$ & response to UV, regulation of proteolysis \\
\hline KCNK3 & 1.3 & $65.1^{*}$ & potassium ion transport, synaptic transmission \\
\hline EHF & 1.8 & $60.7^{*}$ & multicellular organismal development, cell proliferation \\
\hline SCIMP & -2.2 & $15.0^{*}$ & positive regulation of ERK1 and ERK2 cascade \\
\hline SLC1A2 & 1.0 & $32.2^{*}$ & ion transport, synaptic transmission \\
\hline INHBE & 1.2 & $35.8^{*}$ & growth \\
\hline CD274 & -1.4 & $20.0^{*}$ & T cell costimulation, positive regulation of $\mathrm{T}$ cell proliferation \\
\hline NR4A3 & 1.4 & $38.5^{*}$ & $\begin{array}{l}\text { biological_process, transcription initiation from RNA polymerase II } \\
\text { promoter }\end{array}$ \\
\hline NPR1 & 1.5 & $41.3^{*}$ & $\begin{array}{l}\text { positive regulation of renal sodium excretion, positive regulation of } \\
\text { urine volume }\end{array}$ \\
\hline ALDH1A2 & $7.8^{*}$ & $211.9 *$ & $\begin{array}{l}\text { retinoic acid metabolic process, negative regulation of cell } \\
\text { proliferation }\end{array}$ \\
\hline SPINT2 & $-2.3^{*}$ & $10.7^{*}$ & $\begin{array}{l}\text { cellular component movement, negative regulation of } \\
\text { endopeptidase activity }\end{array}$ \\
\hline MMP7 & $3.5^{*}$ & $78.4^{*}$ & extracellular matrix disassembly, extracellular matrix organization \\
\hline VCAM1 & 1.3 & $27.7^{*}$ & $\begin{array}{l}\text { cytokine-mediated signaling pathway, extracellular matrix } \\
\text { organization }\end{array}$ \\
\hline WISP1 & 1.0 & $20.9 *$ & cell-cell signaling, signal transduction \\
\hline TFPI2 & $12.1^{*}$ & $242.6^{*}$ & blood coagulation, negative regulation of endopeptidase activity \\
\hline IL13RA2 & -1.1 & $18.3^{*}$ & signal transduction, cytokine-mediated signaling pathway \\
\hline GJB2 & -1.5 & $12.7^{*}$ & transport, gap junction assembly \\
\hline TGM2 & $9.3^{*}$ & $156.1^{*}$ & positive regulation of cell adhesion, apoptotic cell clearance \\
\hline F3 & $-2.4 *$ & $6.7^{*}$ & blood coagulation, blood coagulation, extrinsic pathway \\
\hline ROR1-AS1 & 1.2 & $18.8^{*}$ & NA \\
\hline
\end{tabular}

Note: The values of fold changes indicate the fold change of the gene transcription of $M O$ and iDC compared to THP-1 monocyte based on 3 parallel cell-based experiments, ${ }^{*} q<0.05$ indicates the significance calculated by intensity-based moderated t-statistics (IBMT). Top 25 genes are genes for which iDC have the highest difference with MO. 
Table 4.S3 Significantly activated or inhibited upstream regulators in MO and iDC (z-score > 2 or <2)

\begin{tabular}{lll}
\hline Upstream Regulators & M0 (z-score) & iDC (z-score) \\
\hline ECSIT & 3.47 & 3.74 \\
CD40LG & 2.77 & 3.25 \\
TNF & 2.68 & 3.29 \\
TLR4 & 2.38 & 3.13 \\
CD40 & 2.40 & 2.78 \\
IL17A & 2.42 & 2.61 \\
IL6 & 2.40 & 2.40 \\
TNFRSF1A & 2.20 & 2.42 \\
TREM1 & 1.63 & 2.12 \\
GAPDH & N/A & 2.22 \\
TSLP & N/A & 2.21 \\
mir-155 & -2.24 & -2.24 \\
mir-146 & -2.21 & -1.98 \\
TGFB1 & N/A & -2.20 \\
LY6E & -1.96 & -2.18 \\
\hline
\end{tabular}

Note: $z$-score was calculated by IPA which represents the bias in gene regulation that predicts whether the upstream regulator exists in an activated or inactivated state. $z$-score $>2$ (red) or $<-2$ (green) was considered to be significant. 
Table 4.S4 Significantly differentially transcribed genes and their fold change (FC) in H-glu-BLG and W-glu-BLG treated MO

\begin{tabular}{|c|c|c|c|}
\hline Sample & Gene name & FC & Gene Ontology Description \\
\hline \multirow[t]{12}{*}{ H-glu-BLG } & HLA-DQA2 & 2.0 & $\begin{array}{l}\text { cytokine-mediated signaling pathway, antigen processing and } \\
\text { presentation of exogenous peptide antigen via MHC class II }\end{array}$ \\
\hline & AMPD3 & 2.1 & purine nucleobase metabolic process, AMP catabolic process \\
\hline & SLC2A6 & 2.4 & transmembrane transport, glucose transport \\
\hline & SOD2 & 2.6 & $\begin{array}{l}\text { release of cytochrome } \mathrm{c} \text { from mitochondria, vasodilation by } \\
\text { acetylcholine involved in regulation of systemic arterial blood } \\
\text { pressure }\end{array}$ \\
\hline & NCF1C & 3.0 & oxidation-reduction process \\
\hline & CRIM1 & 3.3 & $\begin{array}{l}\text { nervous system development, insulin-like growth factor } \\
\text { receptor signaling pathway }\end{array}$ \\
\hline & EBI3 & 3.6 & humoral immune response, T-helper 1 type immune response \\
\hline & TNFAIP6 & 4.1 & cell-cell signaling, signal transduction \\
\hline & IL18R1 & 9.1 & signal transduction, immune response \\
\hline & ID1 & -3.1 & $\begin{array}{l}\text { angiogenesis, transforming growth factor beta receptor } \\
\text { signaling pathway }\end{array}$ \\
\hline & ID3 & -2.9 & $\begin{array}{l}\text { multicellular organismal development, negative regulation of } \\
\text { transcription, DNA-templated }\end{array}$ \\
\hline & MBNL2 & -2.1 & $\begin{array}{l}\text { regulation of RNA splicing, regulation of alternative mRNA } \\
\text { splicing, via spliceosome }\end{array}$ \\
\hline \multirow[t]{13}{*}{ W-glu-BLG } & SUMO4 & 2.1 & protein sumoylation \\
\hline & TAF7 & 2.1 & $\begin{array}{l}\text { spermine transport, negative regulation of transcription from } \\
\text { RNA polymerase II promoter }\end{array}$ \\
\hline & ACKR3 & 2.1 & $\begin{array}{l}\text { receptor internalization, chemokine-mediated signaling } \\
\text { pathway }\end{array}$ \\
\hline & HLA-DQA2 & 2.2 & $\begin{array}{l}\text { cytokine-mediated signaling pathway, antigen processing and } \\
\text { presentation of exogenous peptide antigen via MHC class II }\end{array}$ \\
\hline & IDO1 & 2.3 & tryptophan catabolic process, female pregnancy \\
\hline & NCF1C & 2.5 & oxidation-reduction process \\
\hline & SOD2 & 2.6 & $\begin{array}{l}\text { release of cytochrome } \mathrm{c} \text { from mitochondria, vasodilation by } \\
\text { acetylcholine involved in regulation of systemic arterial blood } \\
\text { pressure }\end{array}$ \\
\hline & $\mathrm{EBI3}$ & 3.2 & humoral immune response, T-helper 1 type immune response \\
\hline & TNFRSF9 & 3.2 & apoptotic process, negative regulation of cell proliferation \\
\hline & CRIM1 & 3.3 & $\begin{array}{l}\text { nervous system development, insulin-like growth factor } \\
\text { receptor signaling pathway }\end{array}$ \\
\hline & TNFAIP6 & 4.1 & cell-cell signaling, signal transduction \\
\hline & TNFSF18 & 4.5 & $\begin{array}{l}\text { T cell proliferation involved in immune response, tumor } \\
\text { necrosis factor-mediated signaling pathway }\end{array}$ \\
\hline & IL18R1 & 7.6 & signal transduction, immune response \\
\hline
\end{tabular}


Table 4.S5 Significantly differentially transcribed genes and their fold change (FC) in BLG, H-glu-BLG and W-glu-BLG treated iDC

\begin{tabular}{|c|c|c|c|}
\hline Sample & Gene name & FC & Gene Ontology Description \\
\hline \multirow[t]{4}{*}{ BLG } & TNFAIP6 & 3.7 & cell-cell signaling, signal transduction \\
\hline & TRBV5-5 & 2.4 & NA \\
\hline & SOD2 & 2.2 & $\begin{array}{l}\text { release of cytochrome c from mitochondria, vasodilation by } \\
\text { acetylcholine involved in regulation of systemic arterial blood } \\
\text { pressure }\end{array}$ \\
\hline & LOC101929565 & -2.2 & NA \\
\hline \multirow[t]{8}{*}{ H-glu-BLG } & OR8A1 & 3.2 & $\begin{array}{l}\text { G-protein coupled receptor signaling pathway, axon } \\
\text { guidance }\end{array}$ \\
\hline & CD6 & 2.3 & cell adhesion , receptor-mediated endocytosis \\
\hline & LINC01099 & -2.1 & NA \\
\hline & LOC101929565 & -2.1 & NA \\
\hline & MIR4797 & -2.2 & NA \\
\hline & AKTIP & -2.2 & $\begin{array}{l}\text { positive regulation of protein phosphorylation, positive } \\
\text { regulation of protein binding }\end{array}$ \\
\hline & UGT1A5 & -2.7 & metabolic process \\
\hline & MIR4451 & -3.3 & NA \\
\hline W-glu-BLG & UGT1A5 & -3.2 & metabolic process \\
\hline
\end{tabular}

Table 4.S6 Significantly activated or inhibited upstream regulators in MO incubated with BLG and Lglu-BLG (z-score $>2$ or $<-2)$

\begin{tabular}{lll}
\hline Upstream Regulators & BLG (z-score) & L-glu-BLG (z-score) \\
\hline ECSIT & 3.09 & 2.95 \\
IFNG & 3.09 & 2.75 \\
TLR4 & 3.09 & 2.58 \\
IL27 & 2.40 & 2.18 \\
TLR2 & 2.19 & 1.95 \\
TNF & 2.41 & $\mathrm{~N} / \mathrm{A}$ \\
IL1B & 2.21 & $\mathrm{~N} / \mathrm{A}$ \\
LY6E & -2.43 & -2.43 \\
\hline
\end{tabular}

Note: $z$-score was calculated by IPA which represents the bias in gene regulation that predicts whether the upstream regulator exists in an activated or inactivated state. $z$-score $>2$ (red) or $<-2$ (green) was considered to be significant. 
Table 4.S7 Gene transcription of surface markers in MO and iDC

\begin{tabular}{|c|c|c|}
\hline Phenotypic markers & MO & iDC \\
\hline CD1B & 1.1 & $4.6^{*}$ \\
\hline $\mathrm{CD} 1 \mathrm{C}$ & 2.0 & $12.5^{*}$ \\
\hline CD1E & $1.9^{*}$ & $2.1^{*}$ \\
\hline CD3G & $3.3^{*}$ & -1.4 \\
\hline CD4 & 1.2 & $-3.6^{*}$ \\
\hline CD9 & $42.9 *$ & $20.8^{*}$ \\
\hline CD11c & $15.7^{*}$ & $21.2^{*}$ \\
\hline CD14 & $27.9 *$ & $9.0 *$ \\
\hline $\mathrm{CD} 22$ & $87.9 *$ & $36.1^{*}$ \\
\hline CD36 & $20.6 *$ & $9.4^{*}$ \\
\hline CD38 & $-12.3^{*}$ & $-7.4^{*}$ \\
\hline CD40 & $2.3^{*}$ & $5.4^{*}$ \\
\hline CD44 & $2.7^{*}$ & $6.4^{*}$ \\
\hline CD47 & $2.0^{*}$ & 1.2 \\
\hline CD52 & $27.9 *$ & $41.7^{*}$ \\
\hline CD53 & $7.4^{*}$ & $9.3^{*}$ \\
\hline CD55 & $3.4^{*}$ & $2.7^{*}$ \\
\hline CD58 & $2.4^{*}$ & $1.7^{*}$ \\
\hline CD59 & $4.8^{*}$ & $4.7^{*}$ \\
\hline CD63 & $1.7^{*}$ & $2.0 *$ \\
\hline $\mathrm{CD} 70$ & $-4.6^{*}$ & $-8.5^{*}$ \\
\hline CD79A & -1.5 & $2.1^{*}$ \\
\hline CD81 & $2.5^{*}$ & $2.0^{*}$ \\
\hline CD82 & $2.7^{*}$ & $4.1^{*}$ \\
\hline CD83 & $7.4^{*}$ & $16.1^{*}$ \\
\hline CD84 & $2.1^{*}$ & $3.8^{*}$ \\
\hline CD86 & 1.8 & $9.5^{*}$ \\
\hline CD101 & 1.6 & -2.1 \\
\hline CD109 & $10.1^{*}$ & $2.2^{*}$ \\
\hline CD151 & $2.5^{*}$ & $2.4^{*}$ \\
\hline CD163 & $17.9^{*}$ & $6.6^{*}$ \\
\hline CD164 & $2.3^{*}$ & $2.0^{*}$ \\
\hline CD180 & $9.7^{*}$ & -1.1 \\
\hline CD209 & $5.7^{*}$ & $13.4^{*}$ \\
\hline CD226 & 2.3 & $16.6^{*}$ \\
\hline CD244 & $-2.3^{*}$ & $-6.4^{*}$ \\
\hline $\mathrm{CD} 274$ & -1.4 & $20.0^{*}$ \\
\hline CD276 & $3.6^{*}$ & $4.3^{*}$ \\
\hline CD300a & $5.6^{*}$ & $3.9 *$ \\
\hline CD300lb & $2.8^{*}$ & $26.0^{*}$ \\
\hline CD320 & $-2.1 *$ & -1.5 \\
\hline HLA-A & $2.2^{*}$ & $1.8^{*}$ \\
\hline HLA-B & $1.7^{*}$ & $1.7^{*}$ \\
\hline HLA-C & $2.3^{*}$ & $2.3^{*}$ \\
\hline HLA-DPA1 & $-3.5^{*}$ & $-2.6^{*}$ \\
\hline HLA-DPB1 & $-3.5^{*}$ & $-2.8^{*}$ \\
\hline HLA-DQB1 & $-2.9^{*}$ & $-6.5^{*}$ \\
\hline HLA-DQB2 & $-1.6^{*}$ & $-2.0^{*}$ \\
\hline HLA-DRA & $-12.0^{*}$ & $-8.7^{*}$ \\
\hline HLA-DRB1 & 1.0 & $-4.3^{*}$ \\
\hline HLA-DRB5 & $-3.1^{*}$ & $-5.8^{*}$ \\
\hline HLA-DRB6 & $1.9 *$ & 1.2 \\
\hline
\end{tabular}

Note: The values indicate the surface markers' gene transcription fold changes of THP-1 derived MO and iDC compared to their control (exposed to culture medium) of $N=3$ experiments, ${ }^{*} p<0.05$. Only genes which has significant transcription level in at least one cell type were shown in the table. 


\section{Chapter 5}

Hydrophobicity drives receptormediated uptake of heat-processed proteins by THP- 1 macrophages and dendritic cells, but not cytokine responses

Ying Deng, Coen Govers, Malgorzata Teodorowicz, leva Liobyte, llaria De Simone, Kasper Hettinga and Harry J. Wichers 
Abstract: Impact of processing on immunogenicity of food proteins has clearly been demonstrated, but the underlying mechanisms are still unclear. In our previous study, the uptake of the cow's milk protein $\beta$-lactoglobulin (BLG) by THP-1 macrophages differed after different processing methods and was positively correlated with hydrophobicity and aggregation. Here we applied the same 3 processing methods: wet heating $\left(60^{\circ} \mathrm{C}\right)$ and low- or high-temperature $\left(50^{\circ} \mathrm{C}\right.$ or $130^{\circ} \mathrm{C}$, respectively) dry heating in absence or presence of reducing sugars (i.e. glucose, lactose or GOS) to lysozyme and thyroglobulin, which have different pl or molecular weight compared to BLG, respectively. Uptake was tested in two types of, genetically homogeneous, antigen-presenting cells (APCs; being macrophages and dendritic cells derived from THP-1 monocytes). This revealed a strong correlation between the uptake of the different protein samples by macrophages and dendritic cells, and confirmed the key role of hydrophobicity, over aggregation, in determining the uptake. Several uptake routes were shown to contribute to the uptake of BLG by macrophages. However, cytokine responses following exposure of macrophages to BLG samples was not related to the levels of uptake. Remarkably, heat-treatmentmediated increases in uptake did thus not induce immune responses. Together, our results demonstrate that heat-treatment-induced increased hydrophobicity is the prime driving factor in uptake, but not in cytokine production, by THP-1 macrophages. 


\section{Introduction}

Dietary proteins play an important physiological role, not only in providing amino acids and energy, but also for the maturation and regulation of the immune system. Proteins are known to be involved in the regulation of chronic inflammation and be the cause of allergies [1]. For example, a higher intake of animal protein was found to enhance the pro-inflammatory response of macrophages in mice [2]. Most of the proteins that humans ingest have been through a heating process as part of the food processing, as a result of which structural modifications, such as glycation (with reducing sugar) and/or aggregation of proteins, may occur.

In Chapter 3 study on $\beta$-lactoglobulin (BLG), the major whey protein of milk, we observed that heat processing under different conditions altered the physicochemical properties and its uptake by THP-1 macrophages. In particular, heating in solution at $60{ }^{\circ} \mathrm{C}$ for 3 days introduced changes in hydrophobicity and molecular weight, which were shown to be the key determinants for uptake. This event may be relevant for a possible subsequent immune response $[3,4]$ and has been linked to heattreatment-induced changes in protein's propensity to induce an allergic reaction [5-7]. Despite the new findings in that study, obvious limitations relate to the use of a single protein, cell phenotype and immunological read-out.

Here, we extend previous findings by investigating 2 other proteins (lysozyme and thyroglobulin), another cell phenotype (THP-1 dendritic cells), and different immunological responses to yield novel insight. Lysozyme and thyroglobulin were subjected to the same heat treatments as used for BLG. Lysozyme from chicken egg white is a single chain polypeptide with four disulphide bridges and is a rather inflexible protein with stable structure and properties [8]. It has a molecular weight of $14.3 \mathrm{kDa}$ which is similar to $18.4 \mathrm{kDa}$ of BLG and an isoelectric point (pl) of 10.7 [9] which is clearly different from BLG (pl = 5.2) [10], resulting in a positive net charge of this protein at the physiological pH. Moreover, it is also known as an allergen, as exemplified by the high incidence of sensitization and allergenicity to this protein [11]. On the other hand, bovine thyroglobulin, which originates from follicular cells of the thyroid gland, is a protein that is considered as less allergenic. It has a molecular weight of $330 \mathrm{kDa}$ as a monomer, making it more than 18 times larger than the mass of the other two proteins under investigation. It has a complex quaternary structure composed of four subunits that are disulphide bonded $[12,13]$. Its $\mathrm{pl}(4.6)$ is close to that of BLG, conferring it a similar charge as BLG at the $\mathrm{pH}$ of the exposure medium. We analysed uptake of heat-treated proteins by macrophages and dendritic cells and correlated this to physicochemical characteristics. Furthermore, we investigated the mechanism and receptors that are involved in uptake and identified downstream immunological responses by quantifying cytokine response. 
Taken together, our findings provide insights into the role of protein size and $\mathrm{pl}$ towards physicochemical changes upon food-processing related heat-treatments. This appears to yield generalisable correlations between a protein's physicochemical characteristics and its uptake by antigen-presenting cells (APCs). Finally, we identified routes of uptake and downstream immunological responses which will help to clarify the interaction of protein particles with macrophages and the potential immunoregulatory effect of it.

\section{Materials and methods}

\section{Chemicals}

All chemicals were purchased from Sigma Aldrich (St Louis, Missouri, USA) unless otherwise stated.

\section{Sample preparation and fluorescent labelling}

$\beta$-Lactoglobulin (BLG) from cow's milk, lysozyme from chicken egg white and thyroglobulin from bovine thyroid were dissolved in sodium phosphate buffer (10 mM, pH 7.4, same below) to a protein concentration of $5 \mathrm{mg} / \mathrm{mL}$. Besides this solution without saccharides, D-glucose (glu), D-lactose (lac), and galacto-oligosaccharide (GOS; Royal FrieslandCampina, Wageningen, the Netherlands) were added to reach a final 1:4 molar ratio of total free amino groups to saccharide reducing ends. Identical heating methods, being high-temperature dry-heating $(H)$, wet-heating $(W)$ and low-temperature dryheating (L), sample preparation and naming were applied to the proteins as indicated in Chapter 3. Fluorescein isothiocyanate isomer I (FITC) labelling was performed as described in Chapter 3.

\section{THP-1 cell culture and differentiation}

The human monocytic leukaemia cell line THP-1 (ATCC, Manassas, Virginia, USA) was differentiated into cells characteristic for resting macrophages (MO) as described by Chanput et al. [14], at a concentration of $1 \times 10^{6}$ cells $/ \mathrm{mL}$ using $100 \mathrm{ng} / \mathrm{mL}$ phorbol-12-myristate-13-acetate (PMA) for a 48 hours stimulation followed by 2 times cell washing with medium and another 48 hours of rest. Using a cell concentration of $0.25 \times 10^{6}$ cells $/ \mathrm{mL}$ and incubation with $20 \mathrm{ng} / \mathrm{mL}$ of IL-4 and $20 \mathrm{ng} / \mathrm{mL}$ of PMA for 4 days, as described by Katayama et al. [15], immature dendritic cells were generated from THP-1 monocytes.

\section{Physicochemical analysis}

The analysis of solubility (protein mass remaining in solution), loss of amino group (OPA method), AGE formation (fluorescent measurement), exposure of hydrophobicity region (ANS method), surface charge (zeta-potential measurement), secondary structure (circular dichroism measurement) and 
aggregation (size exclusion chromatography) were performed using the protocols as described in Chapter 3.

\section{Uptake and blocking of different uptake routes}

Uptake experiments were performed as described in Chapter 3. No significant difference of absolute uptake value has been found for native BLG, thyroglobulin or lysozyme in macrophages or dendritic cells (Figure 5.S1). As there is no bias for the basic uptake capability for the different proteins, all the uptake results were presented relative to their corresponding native protein to be able to compare among different proteins. To determine which route of uptake was involved in a protein's uptake by THP-1-derived cells, the cells were pre-incubated for 30 minutes with DMSO (control) or different inhibitors: $25 \mu \mathrm{g} / \mathrm{mL}$ nystatin (caveolae-dependent uptake inhibitor), $10 \mu \mathrm{g} / \mathrm{mL}$ chlorpromazine (clathrin-dependent uptake inhibitor), $10 \mu \mathrm{g} / \mathrm{mL}$ cytochalasin B (microphagocytosis inhibitor) or all inhibitors combined, dissolved in DMSO. Following incubation, the cells were washed with PBS and incubated for 2 hours with FITC-labelled protein samples. Subsequently, the cells were harvested and measured using flow cytometry as described in Chapter 3. For uptake blocking experiment, $5 \mu$ l trypan blue was added per $100 \mu$ l of cell suspension to quench extracellular FITC signal before flow cytometry measurement especially. Uptake was calculated as either the relative fold change in mean fluorescence intensity (MFI) relative to control, after correcting for FITC labelling efficiency (control = 1 ), or as percentage to control (control $=100 \%$ ).

\section{Soluble receptor for advanced glycation end products (sRAGE), CD36 and Galectin-3 inhibition ELISA}

The experiment was performed according to the method of Liu et al. [16]. As a positive control, soy protein (Bulk Powder, Colchester, UK) was mixed with D-glucose in an equal w/w ratio in $10 \mathrm{mM}$ PBS buffer ( $\mathrm{pH}$ 7.4), to a protein concentration of $10 \mathrm{mg} / \mathrm{mL}$ and heated for 90 minutes at $120{ }^{\circ} \mathrm{C}$. The positive control was diluted in $1.5 \mathrm{mM}$ of sodium carbonate buffer $\mathrm{pH} 9.6$ to $20 \mu \mathrm{g} / \mathrm{ml}$ and used for plate coating by adding $100 \mu \mathrm{L}$ per well to a Nunc MaxiSorp ${ }^{\mathrm{TM}}$ flat-bottom plate (Thermo Fisher, Waltham, Massachusetts, USA) and incubating overnight at $4{ }^{\circ} \mathrm{C}$. Then the plate was washed 3 times with PBS buffer $\mathrm{pH} 7.4$ containing $0.05 \%(\mathrm{v} / \mathrm{v})$ Tween-20. After blocking with $3 \%$ bovine serum albumin (BSA) in PBS for 1 hour at room temperature, the wells were washed 3 times and incubated with $80 \mu \mathrm{L}$ protein samples $(25 \mu \mathrm{g} / \mathrm{mL})$ at $37^{\circ} \mathrm{C}$ for 1 hour more. The samples were preheated at $37^{\circ} \mathrm{C}$ for 45 minutes with recombinant human SRAGE, CD36 or galectin-3 at a concentration of $1,0.5$ or $3 \mu \mathrm{g} / \mathrm{mL}$ (R\&D Systems, Minneapolis, Minnesota, United States) in PBS buffer with 1.5\% BSA and 0.025\% Tween20. For detection, the plate was washed 3 times and incubated with $80 \mu \mathrm{L}$ per well monoclonal mouse 
IgG2B human SRAGE or galectin-3 antibody (R\&D Systems, Minneapolis, Minnesota, United States) at a concentration of $0.2 \mu \mathrm{g} / \mathrm{ml}$ for 30 minutes shaking at room temperature. After washing 4 times the plate was incubated 30 minutes shaking at room temperature with $80 \mu \mathrm{L}$ per well polyclonal goat antimouse HRP-conjugated (DAKO, Glostrup, Denmark) at a concentration $0.25 \mu \mathrm{g} / \mathrm{mL}$. For galectin-3, an additional incubation step with $0.2 \mu \mathrm{g} / \mathrm{ml}$ Streptavidin (SDT, Baesweiler, Germany) $80 \mu \mathrm{L}$ per well for 20 minutes was followed. For CD36, only incubation with goat anti-human IgG/HRP (SouthernBiotech, Birmingham, Alabama, United States) at a concentration of $0.25 \mu \mathrm{g} / \mathrm{ml}$ for 30 minutes shaking at room temperature was used for the detection step. For all receptor inhibition ELISA, the plate was then washed 4 times and $80 \mu \mathrm{L}$ per well TMB substrate was added and incubated for 10 minutes before stop the reaction by adding $100 \mathrm{uL}$ of $2 \% \mathrm{HCl}$ per well. The absorbance measured at $620 \mathrm{~nm}$ by an Infinite ${ }^{\circledR} 200$ PRO NanoQuant (Tecan, Männedorf, Switzerland) was subtracted from the absorbance at $450 \mathrm{~nm}$. Each sample was measured in triplicate and values were averaged. Absorbance of buffer was used as a control and was subtracted from every sample. The absorbance of the control solution without any inhibition was coded by Abscontrol. The percentage of inhibition for samples was equal to (Abscontrol-Abssample)/Abscontrol*100\%.

\section{Cytokine production measurement}

THP-1 macrophages were incubated with $100 \mu \mathrm{g} / \mathrm{mL}$ of protein sample or medium as control for 16 hours, after which the supernatant was collected. The cytokine production was measured using the ELISA Deluxe Set Human IL-8/CCL20/IL-1ß (Biolegend, San Diego, California, United States), following the manufacturer's protocol.

\section{Statistics}

The statistical analysis was performed using Prism 6 software (GraphPad Software, San Diego, California, United States) with $p<0.05$ considered to be significant. The correlation analysis was done by calculating the Pearson correlation coefficient $(r)$ and the two-tailed $p$ value. The PCA plot was generated with the dataset of variables after being mean centred and weighted by $1 /$ standard deviation using Unscrambler software (CAMO, Oslo, Norway).

\section{Results}

Wet-heating significantly increased uptake of thyroglobulin by THP-1-derived macrophages whereas heat-treatment did not increase uptake of lysozyme

Thyroglobulin and lysozyme were wet-heated (W), high-temperature dry-heated (H) or lowtemperature dry-heated (L) in the absence or presence of saccharides with different lengths: 
monosaccharide (glucose), disaccharide (lactose) or oligosaccharide (GOS). Soluble protein from each sample was concentration calibrated, fluorescently labelled and incubated with resting macrophages that were derived from THP-1 monocytes. High-temperature dry-heating of thyroglobulin in the presence of glucose and lactose and lysozyme in the presence of glucose led to complete insolubility (Figure 5.S2A and 5.S4A) and therefore these samples were excluded from further analysis. Prior to the experiment, the lipopolysaccharide (LPS) contamination was measured (Table 5.S1) and found to be below the threshold level that significantly affected uptake capacity of THP-1 macrophages (i.e. $1000 \mathrm{ng} / \mathrm{mL}$ ), as reported in Chapter 3.

For uptake of thyroglobulin samples by macrophages (Figure 5.1A), the type of heat treatment appeared to play a more determining role than the presence or absence of saccharides. Notably, all wet-heated samples, regardless of the presence of saccharides, showed significantly increased uptake compared to untreated protein. Although not significantly differing from untreated protein, high- and low-temperature dry-heating increased the uptake of thyroglobulin as well. On the contrary, no significant differences in uptake of lysozyme after different treatments and saccharide exposures were noticeable (Figure 5.1B).

A

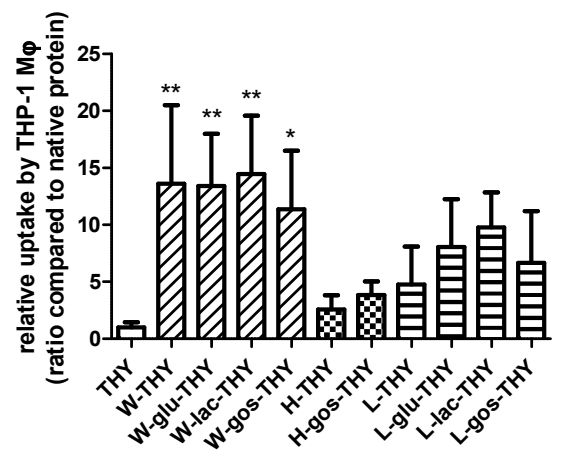

B

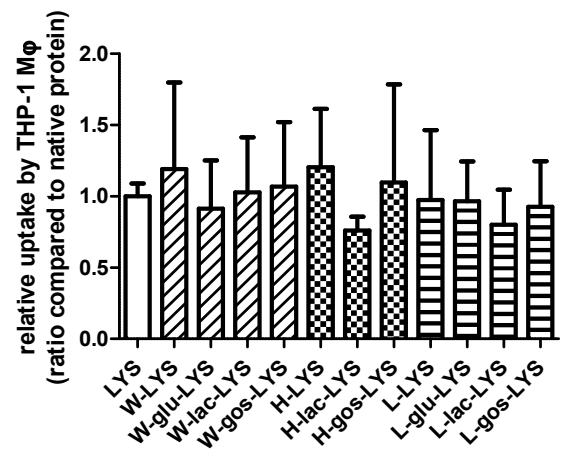

Figure 5.1 Uptake of thyroglobulin by macrophages was significantly increased upon wet-heating of the sample. Thyroglobulin (THY; A) and lysozyme (LYS; B) were untreated or heated $(W, H$ or $L)$ in the absence or presence of saccharides (glu, lac or GOS). THP-1-derived macrophages (M $\phi$ ) were incubated with FITC-labelled protein samples for 2 hours after which uptake was determined using flow cytometry. The results represent mean values $\pm S D$ of $N=4$ measurement of 2 independent experiments based on 2 independent sample sets. Statistical differences compared to native proteins were calculated with Dunnett's Test: ${ }^{*} p<0.05 ;{ }^{* *} p<0.01$. 
Uptake of heat-treated protein samples by THP-1-derived immature dendritic cells is qualitatively similar to that of THP-1-derived macrophages

The uptake of processed thyroglobulin and lysozyme, as well as BLG which was reported in Chapter 3, was only tested for macrophages. To expand the understanding of the proteins' uptake beyond only macrophages, THP-1-derived immature dendritic cells (iDC) were also tested, with identical methodology as for macrophages. The uptake of wet-heated thyroglobulin in the absence or presence of saccharides by these iDC was significantly increased compared to the native protein (Figure 5.2A). For lysozyme, the relative uptake remained the same regardless of the treatment (Figure 5.2B). The uptake of wet-heated BLG in the absence or presence of saccharides by iDCs was also increased compared to the native form, although only reaching significance when heat-treated in the presence of glucose (Figure 5.2C). When compared to the results obtained with macrophages, the increase in uptake upon protein wet-heating for iDC was less pronounced (BLG-macrophage data from Chapter 3). However, the uptake pattern of all samples correlated strongly $(r>0.8, p<0.0001)$ between the two cell types (Figure 5.2D).

A

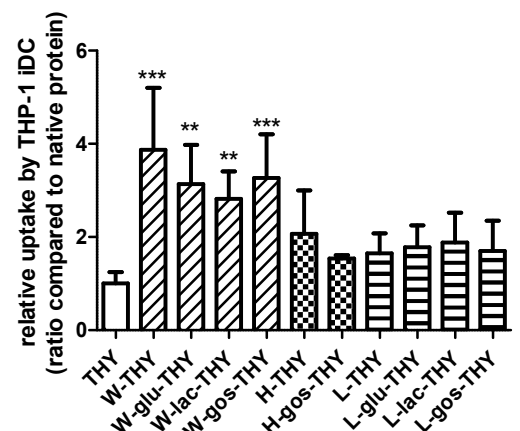

C

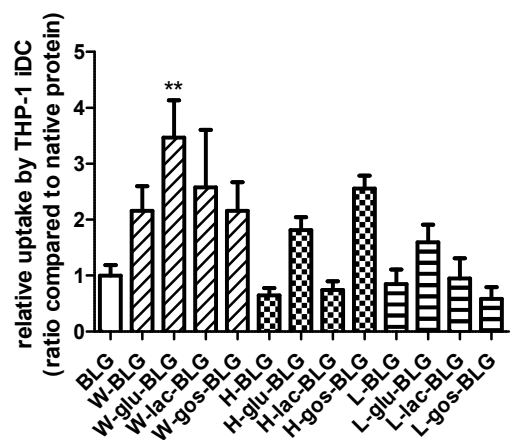

B

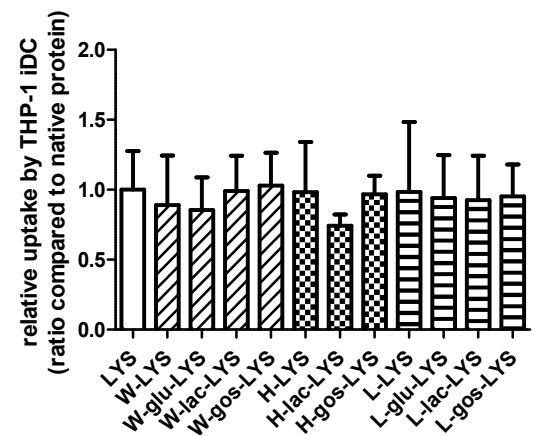

$\mathrm{D}$

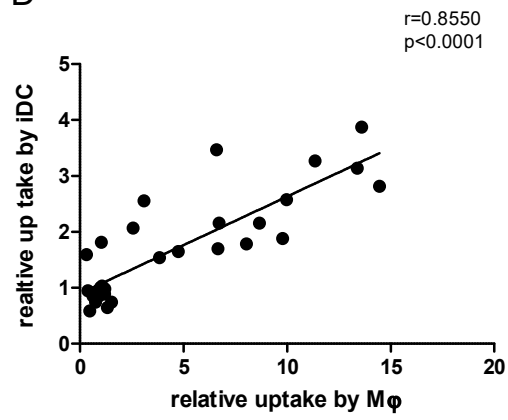


Figure 5.2 Uptake of thyroglobulin, lysozyme and BLG samples by THP-1-derived iDC was strongly and significantly correlated with uptake by macrophages. Figure A-C: Thyroglobulin (THY), lysozyme (LYS) and BLG were untreated or heated $(W, H$ or $L$ ) in the absence or presence of saccharides (glu, lac or GOS). THP-1-derived IDC were incubated with FITC-labelled protein samples for 2 hours after which uptake was determined using flow cytometry. The results represent the mean values $\pm S D$ of $N=4$ measurement of 2 independent experiments based on 2 independent sample sets. Statistical differences compared to native proteins were calculated with Dunnett's Test: ${ }^{* *} p<0.01 ;{ }^{* * *} p<0.001$. Figure $D$ : the relative uptake values of all protein samples by iDC were plotted against macrophages $(M \phi)$ and the correlation analysis was done by calculating the Pearson correlation coefficient ( $r$ ) and two-tailed $p$ value.

\section{Increased uptake of thyroglobulin by THP-1-derived macrophages was correlated to hydrophobicity and aggregation}

Due to the strongly correlated responses of iDC and macrophages in uptake, with a higher relative uptake by macrophages, the latter was used for further testing. To explain the physicochemical mechanism behind the differing uptake capability of macrophages for differently treated thyroglobulin and lysozyme, a set of measurements was performed on the soluble fraction after treatment.

For thyroglobulin, dry-heating at high-temperature significantly decreased its solubility, resulting in the absence of a soluble fraction when heating was performed in the presence of glucose and lactose (Figure 5.S2A). The soluble fraction of samples in the presence of GOS or without saccharide showed a strong significant decrease in free amino groups (Figure 5.S2B). The former sample also showed significant increases in both AGE-related fluorescence and formation of polymers (Figure 5.S2C and 5.S3). Wet-heating did not result in loss of solubility, but also led to formation of polymers and, albeit less pronounced as for high-temperature dry-heating significant, losses of amino groups and increases in AGE-related fluorescence. Moreover, wet-heating induced a significant increase in the exposure of hydrophobic regions as measured by ANS-binding (Figure 5.S2D). Dry-heating at low-temperature did not have much influence on the measured parameters for thyroglobulin, except for a significant loss of amino groups and a slight increase in oligomer and polymer formation (Figure 5.S2B and Figure 5.S3). There was no significant change in the secondary structure for any of the treated thyroglobulin samples (Figure 5.S2E and 5.S2F).

Applying identical heating and glycation conditions to thyroglobulin did not generate any soluble aggregates for lysozyme (size exclusion chromatography, data not shown). High-temperature dryheating led to a significant decrease of solubility with complete insolubility for the samples heated in 
the presence of glucose (Figure 5.S4A). Among the soluble fraction of the high-temperature dry-heated samples, there was a significant decrease of free amino groups, increases of AGE-related fluorescence and exposure of hydrophobic regions in the presence of saccharides (Figure 5.S4B-D). Moreover, results showed a significant loss of $\beta$-sheet structure for all samples except when heated in the presence of GOS (Figure 5.S4F). Similarly, low-temperature dry-heating resulted in a significant loss of $\beta$-sheets in all lysozyme samples. For wet-heated lysozyme, only the exposure of hydrophobic regions in the presence of saccharides was significantly increased (Figure 5.S4D).

To identify the most important physicochemical properties of heat-processed thyroglobulin that are related to its uptake by macrophages, we performed a principle component analysis (PCA) and a correlation analysis. In the PCA plot (Figure 5.3). All low-temperature dry-heated samples clustered together with the untreated thyroglobulin, indicating that the physicochemical properties of these samples were similar. Uptake was strongly related with wet-heating and positively related to hydrophobicity (ANS) and polymer proportion, and inversely to monomer and oligomer proportion. These relations were further confirmed by correlation analyses. As shown in Figure 5.4, there was a strong correlation between uptake and hydrophobicity and a moderate correlation between uptake and aggregation, as indicated by the fraction of monomers, polymers and oligomers. Of note is that the samples were strongly clustering into two groups at the extremes of the data scale for both the uptake and monomer or polymer content correlation plots (Figure 5.4B and 5.4C); the correlation between uptake and oligomer content was less profound (Figure 5.4D, p > 0.05). Contrastingly to thyroglobulin, no clear correlation could be found between treatment and physicochemical properties for lysozyme in the PCA plot (Figure 5.S5). 


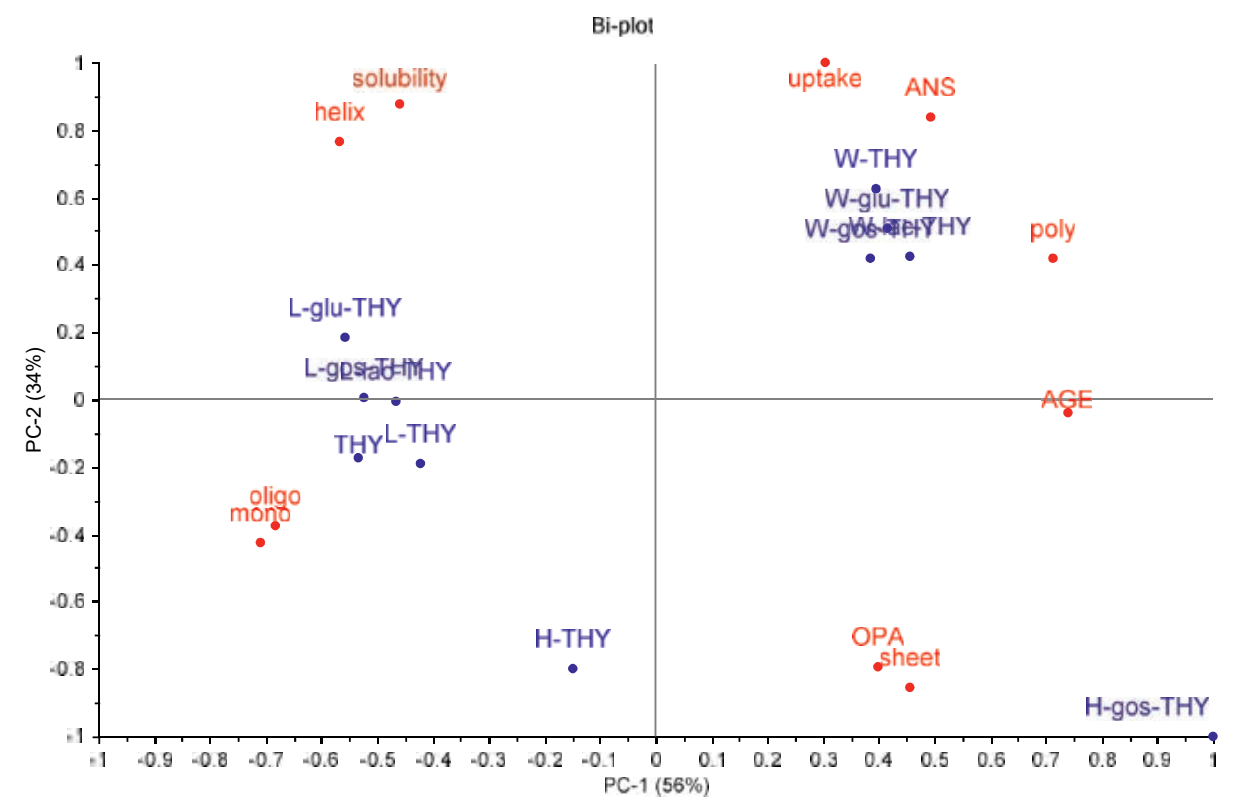

Figure 5.3 Uptake of thyroglobulin by macrophages is related to wet-heating, hydrophobicity and aggregation. Thyroglobulin (THY) was untreated or heated (W, $H$ or $L$ ) in the absence or presence of saccharides (glu, lac or GOS) and tested for solubility, uptake by THP-1 macrophages (uptake), AGE formation (AGE), glycation (OPA), percentage of $\alpha$-helix (helix) or 6-sheet (sheet), aggregation (monomer and smaller (mono), oligomers (oligo), polymers (poly)) and exposure of hydrophobic regions (ANS). PCA scores of the samples were given in blue and the parameter loadings of the principal components were given in red. 
A

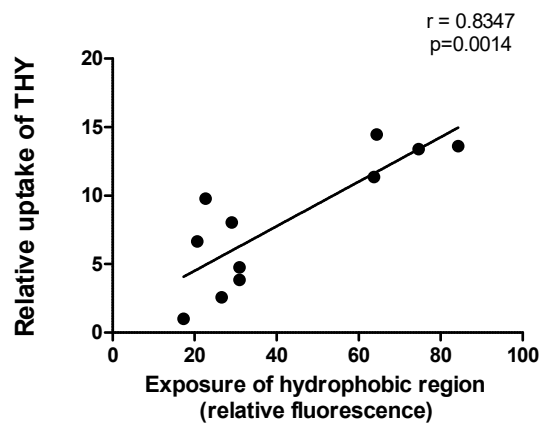

C

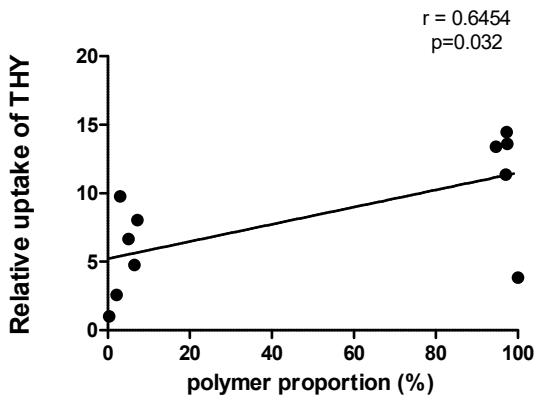

B

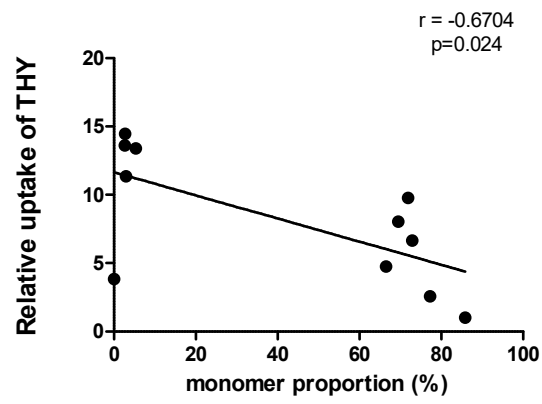

$\mathrm{D}$

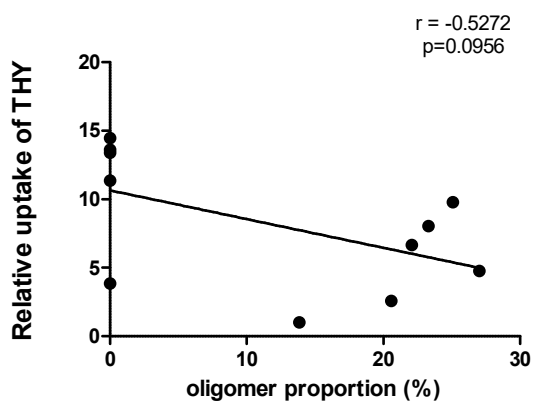

Figure 5.4 Correlation analysis of uptake of heat-treated thyroglobulin with physicochemical parameters. Uptake of thyroglobulin (THY) by macrophages was correlated to hydrophobicity $(|r|>$ $0.7)$ and proportion of monomer, polymer and oligomer $(0.7>|r|>0.5)$. The correlation analysis was done by calculating the Pearson correlation coefficient ( $r$ ) and two-tailed $p$ value.

\section{EDC-mediated cross-linking or increased hydrophobicity demonstrates that hydrophobicity} is the main factor driving uptake by macrophages

EDC links the carboxyl and amino group of amino acids, and thereby offers the possibility to crosslink proteins without heating. This allows for a discrimination between aggregation and other heatingrelated effects, like hydrophobicity changes, with regard to their impact on uptake. As shown in Figure 5.5A and 5.5B, the proportion of polymer and monomer significantly increased, resp. decreased, in the EDC cross-linked BLG and thyroglobulin. On the contrary, only limited aggregation of lysozyme was observed upon EDC-treatment. Surprisingly, EDC significantly increased the hydrophobicity of lysozyme (Figure 5.5C) and also its uptake by macrophages (Figure 5.5D). 
A

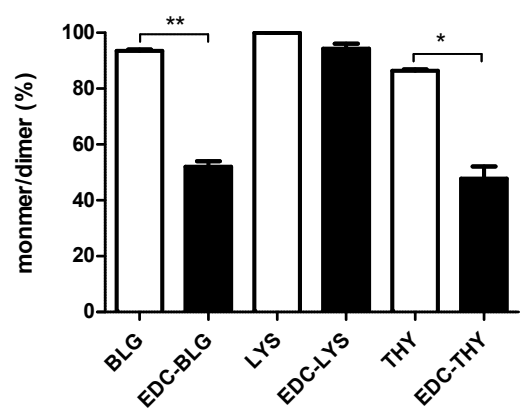

C

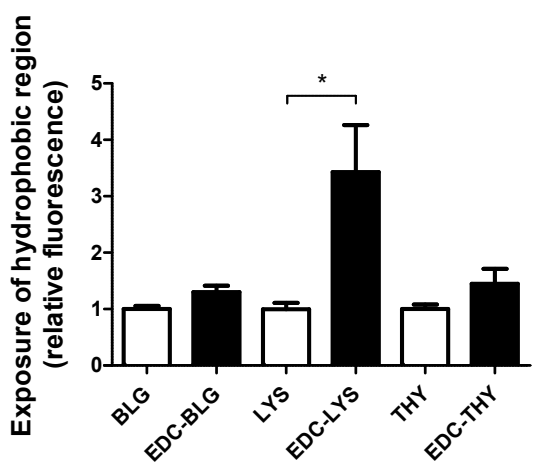

B

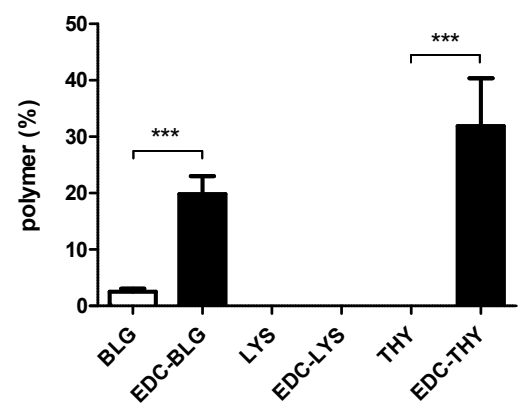

D

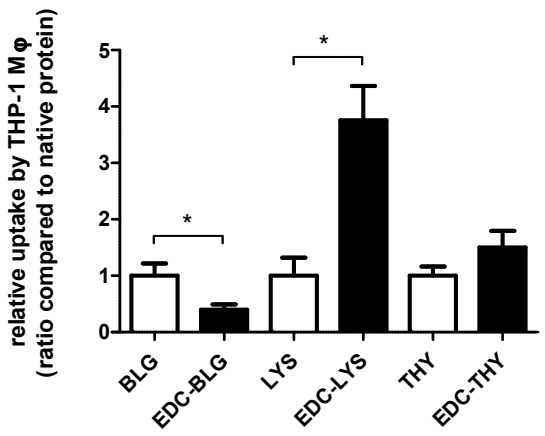

Figure 5.5 Increased hydrophobicity appears to be the dominant factor to drive uptake of proteins. Lysozyme was crosslinked limitedly after EDC treatment based on SEC analysis but caused a significant increase in both its hydrophobicity and uptake by macrophages. Thyroglobulin (THY), lysozyme (LYS) and BLG were non-treated or treated with EDC and subsequently analysed for the proportion of polymer $(A)$, the proportion of monomer/dimer (B), and protein hydrophobicity (C). EDC-treated and nontreated proteins were fluorescently labelled and their uptake by macrophage $(M \phi)$ was measured $(D)$. The results represent the mean values $\pm S D$ of 3-4 independent experiments with 2 independent sample sets. Statistical differences were calculated with two-tailed unpaired T-test with Welch's correction: *p $<0.05 ; * *<0.01 ; * * p<0.001$.

\section{Uptake of heated-treated BLG was mediated via several routes}

To better understand the mechanism of uptake, we set out to identify which routes of uptake were utilized for the various proteins that differed in physicochemical characteristics and therefore in their recognition motifs for cells. Native and heat-treated BLG in the presence of glucose was used as a subset, because these samples covered the whole range of levels of physicochemical modifications. 
Pre-incubation of macrophages with nystatin (caveolae inhibitor) and the combination of inhibitors, prior to BLG samples exposure, significantly inhibited the uptake (Figure 5.6). The usage of cytochalasin (microphagocytosis inhibitor) significantly decreased the uptake for both high-temperature dry-heated and wet-heated BLG (Figure 5.6B and 5.6C). Moreover, chlorpromazine did significantly reduce uptake of wet-heated BLG, indicating that clathrin was involved in this process (Figure 5.6C).

A

BLG

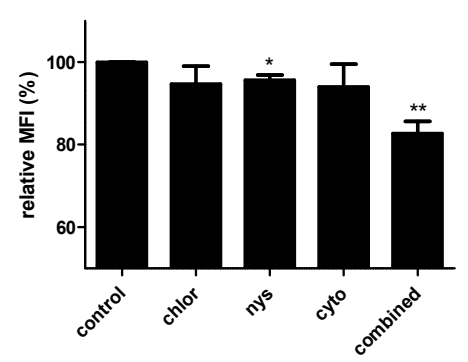

C

W-glu-BLG

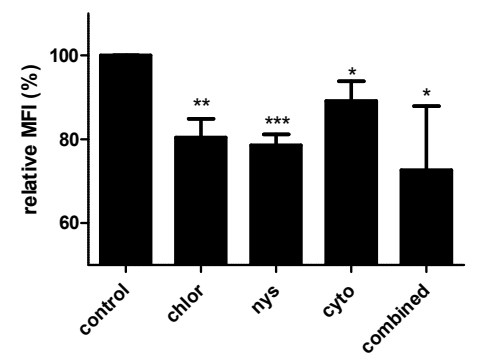

B

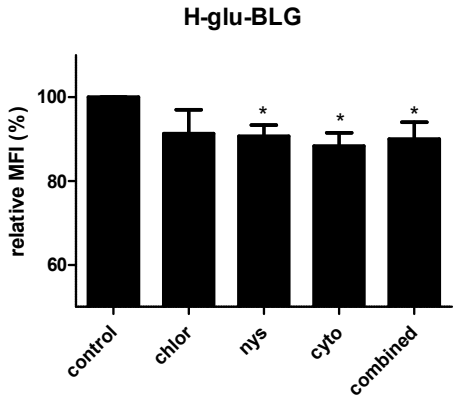

$\mathrm{D}$

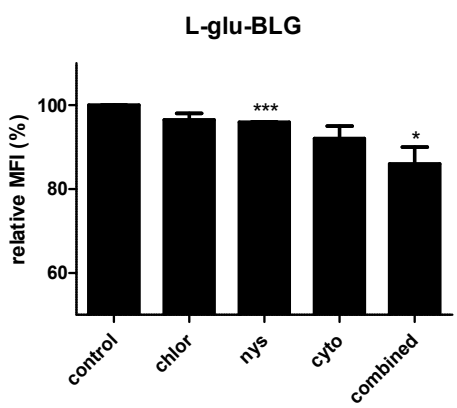

Figure 5.6 Several routes appeared to be involved in the uptake of wet-heated BLG. BLG was untreated or heated (W, H or L) in the presence of glucose (glu) and FITC-labelled. Macrophages were pre-treated with DMSO (control) or inhibitors (dissolved in DMSO) of clathrin-dependent uptake (chlorpromazine; chlor), caveolae-dependent uptake (nystatin; nys), microphagocytosis (cytochalasin; cyto) or a combination of them (combined) for 30 min before a 2 hour incubation with protein samples, then the uptake was measured using flow cytometry. The relative MFI was calculated by dividing the MFI of a sample by the MFI of the corresponding control. The results represent mean values $\pm S D$ of multiple independent cell experiments $(N=2-4)$ with 2 independent sample sets. The statistical differences were calculated with one-tailed unpaired T-test compared to control, ${ }^{*} p<0.05,{ }^{* *} p<0.01,{ }^{* * *} p<0.001$. 
Inhibition ELISA showed high binding of high-temperature dry-heated and wet-heated BLG by sRAGE, CD36 and galectin-3

We investigated several receptors of the cells that might be involved in binding and subsequent uptake of the BLG samples that were heat-treated in the presence of glucose. Using an inhibition ELISA, the binding of the BLG samples to the soluble receptor for advanced glycation end products (sRAGE), CD36scavenger receptor and galectin-3 was analysed (Figure 5.7). Native BLG did not show any binding to SRAGE, galectin-3 or CD36. In contrast, high-temperature dry-heating of BLG induced significant receptor binding for all three tested receptors. Wet-heating of BLG similarly resulted in binding to all three receptors, albeit to a lesser extent and only significantly for sRAGE and galectin-3. Finally, lowtemperature dry-heating did not induce any receptor-binding.

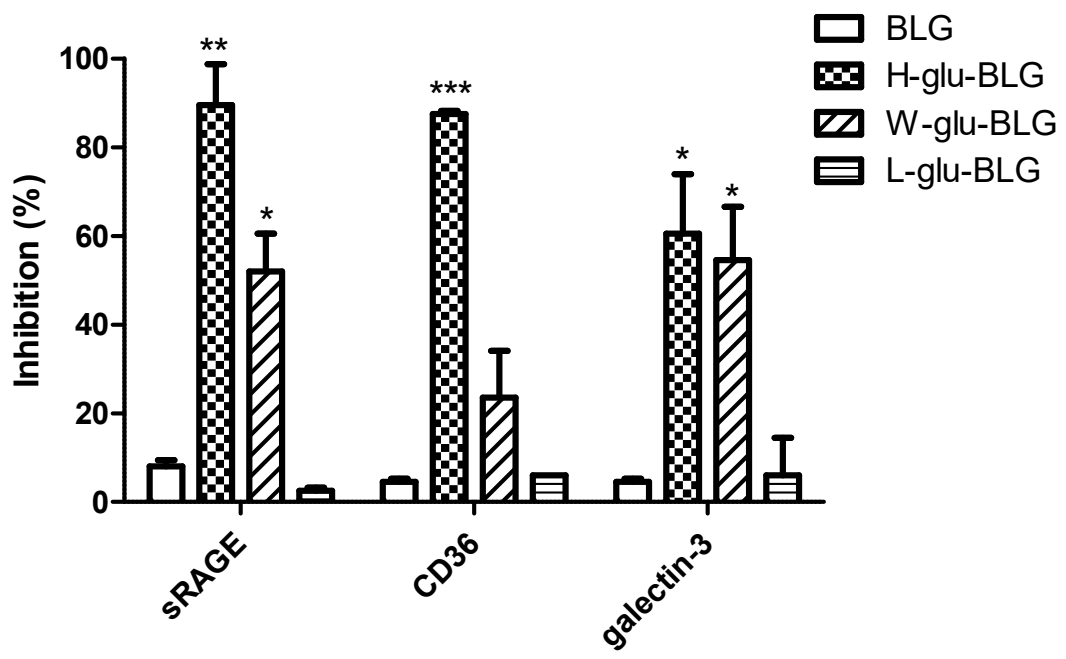

Figure 5.7 Heat-treatment in the presence of glucose induces receptor binding capacity of BLG. BLG was untreated or heated (W, H or L) in the presence of glucose ( $g l u$ ) and was used to inhibit the binding of receptor to a positive control in the inhibition ELISA with a concentration of $25 \mu \mathrm{g} / \mathrm{mL}$. Data shown are the mean values $\pm S D$ of $N=4$ based on measurement of 2 parallel prepared samples. Statistically significant differences between native and processed BLG for each receptor was determined using unpaired two-tailed t-Test, ${ }^{*} p<0.05,{ }^{* *} p<0.01,{ }^{* * *} p<0.001$.

Cytokine responses of macrophages following exposure to BLG were reduced upon heattreatment of BLG 
Receptor binding and uptake of protein samples can lead to immune activity. The immunological response could be estimated by the expression of secreted cytokines or chemokines. Upon exposure of medium to native or heat-treated BLG in the presence of glucose, the response of macrophages was analysed by measuring the levels of secreted IL-8, CCL20 and IL-1ß. As shown in Figure 5.8, nonprocessed native BLG induced significant levels of IL-8, CCL20 and IL-1 $\beta$ secretion in macrophages. High-temperature dry-heating of BLG significantly reduced the secretion of all three analysed cytokines. A similar reduction was observed in wet-heated BLG for IL-8 and IL-1 3 , but less strongly and not significantly for CCL20. There is no clear difference between native and low-temperature dry-heated BLG in stimulating macrophages' cytokine secretion.

A

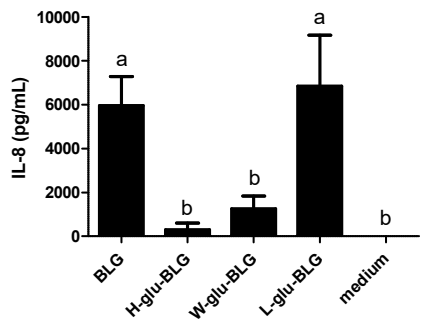

B

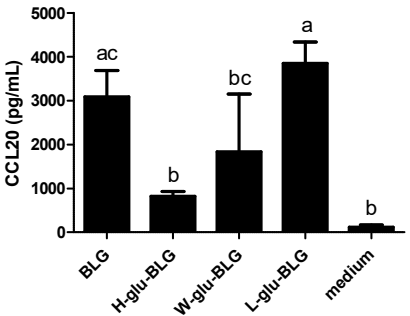

C

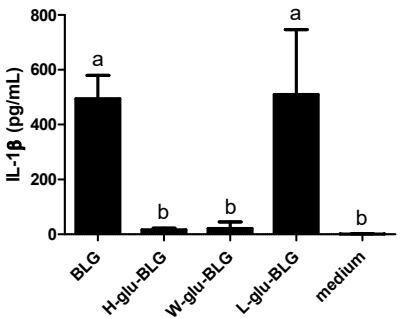

Figure 5.8 Cytokine and chemokine responses of macrophages upon exposure to native or heat-treated $B L G$ varies. BLG was untreated or heated (W, $H$ or $L)$ in the presence of glucose and incubated with macrophages. The cytokine secretion of IL-8 (A), CCL2O (B) and IL-1B (C) was analysed in the supernatant with ELISA. The results represent mean values $\pm S D$ of 2-4 independent experimental measurements. The statistical differences were calculated with Tukey's Test and means with different letters $(a-c)$ in the bars indicated significant difference $(p<0.05)$.

\section{Discussion}

In earlier study of Chapter 3, we observed that hydrophobicity and the aggregation state of heatprocessed BLG were important parameters that were related to the protein uptake by THP-1 macrophages. Here, we extended these findings by analysing whether similar relations would apply for 1) a protein with similar pl but higher molecular weight (thyroglobulin); 2) a protein with similar molecular weight but higher pl (lysozyme); 3) EDC-aggregated proteins; and 4) another cell type by using THP-1 dendritic cells. The comparison between THP-1-derived macrophages and dendritic cells regarding uptake revealed similar patterns for the three tested proteins and the different heattreatments. Macrophages and DCs are both able to capture exogenous particles and induce an immunogenic response, playing an essential role in immunological defence [17]. We did observe, 
however, a quantitative difference in uptake, as the uptake values for wet-heated thyroglobulin and BLG were relatively higher in macrophages than in DCs. This suggests that macrophages are more efficient in their phagocytic role than DCs, which is in line with literature stating that uptake by macrophages is part of their core role in eliminating harmful substances, whereas uptake by DCs is a mean to initiate an adaptive immune response [18]. Upon comparing the three tested proteins, we found that the physicochemical alterations upon heat-treatment to thyroglobulin and BLG were similar through different from lysozyme. These proteins differ in size (thyroglobulin (330 kDa) > BLG (18.4 kDa) lysozyme (14.3 kDa)), pl (lysozyme (10.7) > BLG (5.2) thyroglobulin (4.5)) and denaturation temperature (lysozyme $\left(75^{\circ} \mathrm{C}\right)>\mathrm{BLG}\left(65^{\circ} \mathrm{C}\right) \sim$ thyroglobulin $\left(60^{\circ} \mathrm{C}\right)$ ) [19-22], suggesting that both $\mathrm{pl}$ and heat stability may be important for heat-induced structural protein changes. The high pl may have conferred a positive charge to lysozyme during wet-heating $(\mathrm{pH} 7.4)$, thereby hindering aggregation under this condition due to electrostatic repulsion. The strong internal coherence and stability of lysozyme's structure may also have contributed to its stability during heat processing [23]. It is reported that the addition of the saccharides would impact the physicochemical characteristics, in particular in combination with the different applied heat-treatments [24], but we found only a very limited influence from the presence of saccharide on the protein characteristics.

Significant increases in uptake of thyroglobulin by macrophages correlated with increases in hydrophobicity and aggregation that occurred upon wet-heating (Figure 5.3 and 5.4), similar to BLG. This is in line with the findings on lysozyme, where the lack of uptake of lysozyme by macrophages may have been related to the lack of aggregation and only minimal increases in hydrophobicity upon heattreatment. In order to better distinguish between aggregation and hydrophobicity as drivers of uptake, we used EDC to chemically crosslink proteins through the carboxyl and amino groups of amino acids (Figure 5.5), thereby being able to generate protein aggregates without applying a thermal treatment. Polymerisation was induced for BLG and thyroglobulin, as expected. Unexpectedly, EDC was unable to induce polymerisation in lysozyme, but it increased its hydrophobicity instead. This might be due to the intramolecular crosslinking of lysozyme between Lys-13 and Leu-129, as described by Yamada et al. [25], upon EDC treatment. In contrast to intermolecular crosslinking, which occurs for most proteins upon EDC treatment, the induction of intramolecular linkages might lead to protein unfolding resulting in exposure of hydrophobic regions. The strong increase in uptake of EDC-treated lysozyme indicates that hydrophobicity is a more important driver for uptake of heat-treated proteins than aggregation. A correlation between hydrophobicity of peptides and uptake by HeLa cells was also reported earlier by Sakagami et al. [26]. Heating of proteins, through its resulting increased exposure of hydrophobicity, may thus underlie the increased uptake by APCs. 
Next, we aimed to elucidate the uptake route of BLG samples by macrophages. Uptake could be categorized into clathrin-dependent and clathrin-independent. In clathrin-dependent uptake, also known as receptor-dependent uptake, cells recognise and internalize the receptor-bound substance [27]. Clathrin-independent uptake functions via caveolae structures (membrane domains) or actin (taking up large particles up to $5 \mu \mathrm{m}$ ) [28]. Uptake mediated by the CD36 receptor was reported to be clathrin-dependent, while galectin-3 and SRAGE are thought to be clathrin-independent [29-31]. Caveolae are reported to be involved in the uptake by APCs [32]. Caveolae-dependent uptake might be the basic route for the uptake of BLG by macrophages as the inhibition of this route led to a reduced uptake for all samples (Figure 5.6). Uptake of wet-heated BLG by macrophages seemed to involve other endocytosis routes (Figure 5.6C). The blocking of microphagocytosis led to a significant decrease in uptake of wet-heated and also high-temperature dry-heated BLG, probably due to the presence of higher molecular weight particles. The clathrin-dependent route might also be involved in the uptake of wet-heated BLG by macrophages, as a significant decrease was found when the respective inhibitor was used. As shown in Figure 5.7, wet-heated BLG in the presence of glucose had clearly higher binding capacity for SRAGE, galectin-3 and CD36 compared to native BLG. However, it is doubtful if these receptors do play essential roles in the significantly enhanced uptake of wet-heated BLG, as these receptors are mainly reported to be involved in the uptake of glycated proteins [33-35], which is not abundant in wet-heated BLG as shown in our previous study in Chapter 3. The binding of wet-heated BLG to the receptors may be due to the high hydrophobicity of the sample. For example, the strong affinity of the S100 protein for SRAGE was ascribed to a binding mechanism in which hydrophobic structures are involved $[36,37]$. High-temperature dry-heated BLG, which had a high degree of glycation, bound to all receptors to a higher extent than wet-heated BLG, but its uptake was not significantly reduced when clathrin-dependent uptake was blocked. This may be due to the fact that the initial uptake efficiency of high-temperature dry-heated BLG was already low, similar to native and low-temperature dry-heated BLG. In another study, the uptake of BLG that had been heated in solution at $60{ }^{\circ} \mathrm{C}$ for 10 days with glucose by dendritic cells was reported to be SRAGE independent but scavenger receptor dependent [38]. Scavenger receptor $A$ was reported to be involved in the uptake of glycated ovalbumin by macrophages [39], which could thus be a potential target for future discrimination. The increased uptake of wet-heated BLG might be due to enhancing of the clathrin/receptor-dependent route, although the involved receptors still need to be clarified.

IL-8, CCL20 and IL-1 $\beta$ are proinflammatory cytokines secreted by macrophages when they are activated by antigens [40]. However, there seems to be no direct relation between the macrophage's cytokine release and its uptake of BLG. As shown in Figure 5.8, the cytokine production of macrophages treated with native and low-temperature dry-heated BLG was generally significantly higher than for the other 
samples. The strong cytokine release of macrophages in response to native BLG exposure might (partly) be related to the higher levels of LPS contamination in this sample, as shown in Figure 5.S1. Native BLG had an LPS concentration of $155 \mathrm{pg} / \mathrm{mL}$, while wet-heated, low- and high-temperature dry-heated BLG in the presence of glucose contained $2.3,7.8$ or $4.6 \mathrm{pg} / \mathrm{mL}$, respectively. A previous study demonstrated that $500 \mathrm{pg} / \mathrm{mL}$ LPS induced a 7-fold increased IL-8 production of THP-1 macrophages compared to the medium [41]. Here, we found a production of $6,000 \mathrm{pg} / \mathrm{mL}$ of IL-8 for macrophages treated with BLG compared to no production with medium. Thus, although the cytokine production of macrophages due to BLG exposure might be partly endotoxin-driven, the BLG-induced IL-8, and also CCL20 and IL-1 $\beta$, production is much higher than would be expected from the level of LPS contamination alone. When comparing the samples, the minor structural changes following lowtemperature dry-heating of BLG did not change the levels of cytokine responses. In contrast, hightemperature dry-heated or wet-heated BLG, with significant structural changes compared to native $B L G$, only induced limited cytokine responses by macrophages. This is in line with findings from another study where BLG-induced production of cytokines by murine bone-marrow derived dendritic cells was also reduced upon exposure to heated BLG, which these authors claimed to be due to glycation. However, in this study BLG was heated in solution with glucose for 10 days at $60^{\circ} \mathrm{C}$, similar to our wetheating process. This makes it likely that also in their samples, physicochemical changes such as increased hydrophobicity, rather than glycation, led to a lowered cytokine response [38]. Increased hydrophobicity of our BLG samples did not induce clear immunological responses but rather lowered these. Seong and Matzinger [28] hypothesized hydrophobic structures ('hyppos') as molecular patterns that initiate immune responses (DAMPs), playing a role in aetiology of certain pathologies. Although we found that hydrophobicity was strongly correlated to uptake of protein (aggregates), we could not establish a direct link with immunogenicity in our strictly in vitro experimental models. Considering the differences in types of proteins that were included in our study vs. the endogenously present, malformed proteins that are the basis of Seong and Matzinger's hypothesis, it is perhaps too early to correlate our findings to the DAMP-concept.

\section{Conclusion}

In conclusion, the uptake of proteins by macrophages and dendritic cells showed strong similarities, indicating that the protein uptake capacity is, to a considerable extent, independent of the phenotype of APCs. Processed thyroglobulin shares characteristics of physicochemical changes and THP-1 macrophage uptake properties with processed BLG. Taking also the data of EDC crosslinked proteins into account, it seems that hydrophobicity is the most important determining factor for uptake. Receptor-dependent uptake might contribute to the enhanced uptake of wet-heated BLG by macrophages whereas the exact involved receptors are not clear yet. The uptake of processed BLG by 
macrophages appears to be insufficient to directly correlate with their potential immunogenicity, as the cytokine secretion pattern of macrophages is not in line with the uptake pattern. Nevertheless, the obtained data showed clearly that there are immunological consequences of physicochemical modifications due to different heating methods, which is of relevance to the food industry in developing new methods to minimize possible adverse immunological effects of processed food. 


\section{References}

1. Gaffney, R.M. and J.R. Casleysmith, Excess Plasma-Proteins as a Cause of Chronic Inflammation and Lymphoedema - Biochemical Estimations. Journal of Pathology, 1981. 133(3): p. 229-242.

2. Kostovcikova, K., et al., Diet Rich in Animal Protein Promotes Pro-inflammatory Macrophage Response and Exacerbates Colitis in Mice. Frontiers in Immunology, 2019. 10.

3. Jorritsma, S.H.T., et al., Delivery methods to increase cellular uptake and immunogenicity of DNA vaccines. Vaccine, 2016. 34(46): p. 5488-5494.

4. Unanue, E.R. and B.A. Askonas, Persistence of Immunogenicity of Antigen after Uptake by Macrophages. Journal of Experimental Medicine, 1968. 127(5): p. 915-\&.

5. Stojadinovic, M., et al., Cross-Linking of beta-Lactoglobulin Enhances Allergic Sensitization Through Changes in Cellular Uptake and Processing. Toxicological Sciences, 2014. 140(1): p. 224-235.

6. Guillon, B., et al., Heat processing of peanut seed enhances the sensitization potential of the major peanut allergen Ara $h$ 6. Molecular Nutrition \& Food Research, 2016. 60(12): p. 27222735.

7. Moghaddam, A.E., et al., Dry roasting enhances peanut-induced allergic sensitization across mucosal and cutaneous routes in mice. Journal of Allergy and Clinical Immunology, 2014. 134(6): p. 1453-1456.

8. Faccio, G., From Protein Features to Sensing Surfaces. Sensors, 2018. 18(4).

9. Abeyrathne, E.D.N.S., H.Y. Lee, and D.U. Ahn, Sequential separation of lysozyme, ovomucin, ovotransferrin, and ovalbumin from egg white. Poultry Science, 2014. 93(4): p. 1001-1009.

10. Majhi, P.R., et al., Electrostatically driven protein aggregation: beta-lactoglobulin at low ionic strength. Langmuir, 2006. 22(22): p. 9150-9159.

11. Fremont, S., et al., Prevalence of lysozyme sensitization in an egg-allergic population. Allergy, 1997. 52(2): p. 224-228.

12. Pitt-Rivers, R., The subunit structure of thyroglobulin. Biochem J, 1976. 157(3): p. 767-8.

13. Edelhoch, H. and B. De Crombrugghe, The properties of thyroglobulin. 13. The structure of reduced alkylated thyroglobulin. J Biol Chem, 1966. 241(19): p. 4357-65.

14. Chanput, W., et al., Characterization of polarized THP-1 macrophages and polarizing ability of LPS and food compounds. Food \& Function, 2013. 4(2): p. 266-276.

15. Katayama, S., et al., Apple polyphenols suppress antigen presentation of ovalbumin by THP-1derived dendritic cells. Food Chemistry, 2013. 138(2-3): p. 757-761.

16. Liu, F.H., et al., Generation of Soluble Advanced Glycation End Products Receptor (sRAGE)Binding Ligands during Extensive Heat Treatment of Whey Protein/Lactose Mixtures is Dependent on Glycation and Aggregation. Journal of Agricultural and Food Chemistry, 2016. 64(33): p. 6477-6486.

17. Ginhoux, F., M. Guilliams, and S.H. Naik, Editorial: Dendritic Cell and Macrophage Nomenclature and Classification. Frontiers in Immunology, 2016. 7.

18. Savina, A. and S. Amigorena, Phagocytosis and antigen presentation in dendritic cells. Immunological Reviews, 2007. 219: p. 143-156.

19. Ui, N., Electrophoretic Mobility and Isoelectric Point of Hog Thyroglobulin. Biochimica Et Biophysica Acta, 1972. 257(2): p. 350-\&.

20. Yan, Y.F., et al., pH-Dependent Aggregation and Disaggregation of Native beta-Lactoglobulin in Low Salt. Langmuir, 2013. 29(14): p. 4584-4593.

21. Pavelkic V. , O.S., Beljanski M. , Vucelic V., and Vucelic D., The thermal stability of $b$ microglobulin and thyroglobulin, in BIBLID. 1998. p. 181-188.

22. Chang, J.Y. and L. Li, The unfolding mechanism and the disulfide structures of denatured lysozyme. Febs Letters, 2002. 511(1-3): p. 73-78.

23. Arai, T. and W. Norde, The Behavior of Some Model Proteins at Solid Liquid Interfaces .2. Sequential and Competitive Adsorption. Colloids and Surfaces, 1990. 51: p. 17-28. 
24. Liang, Y.C., et al., Effect of sugar type and concentration on the heat coagulation of oil-in-water emulsions stabilized by milk-protein-concentrate. Food Hydrocolloids, 2014. 41: p. 332-342.

25. Yamada, H., et al., Intramolecular Cross-Linkage of Lysozyme - Imidazole Catalysis of the Formation of the Cross-Link between Lysine-13 (Epsilon-Amino) and Leucine-129 (AlphaCarboxyl) by Carbodiimide Reaction. Biochemistry, 1983. 22(19): p. 4551-4556.

26. Sakagami, K., et al., Importance of Net Hydrophobicity in the Cellular Uptake of All-Hydrocarbon Stapled Peptides. Molecular Pharmaceutics, 2018. 15(3): p. 1332-1340.

27. Mousavi, S.A., et al., Clathrin-dependent endocytosis. Biochemical Journal, 2004. 377: p. 1-16.

28. Hansen, C.G. and B.J. Nichols, Molecular mechanisms of clathrin-independent endocytosis. Journal of Cell Science, 2009. 122(11): p. 1713-1721.

29. Eckhardt, E.R.M., et al., High density lipoprotein endocytosis by scavenger receptor SR-BII is clathrin-dependent and requires a carboxyl-terminal dileucine motif. Journal of Biological Chemistry, 2006. 281(7): p. 4348-4353.

30. Du Toit, A., ENDOCYTOSIS Bend it like galectin 3. Nature Reviews Molecular Cell Biology, 2014. 15(7).

31. Stitt, A.W., et al., Advanced glycation end-product receptor interactions on microvascular cells occur within caveolin-rich membrane domains. Faseb Journal, 2000. 14(15): p. 2390-2392.

32. Harris, J., et al., Caveolae and caveolin in immune cells: distribution and functions. Trends in Immunology, 2002. 23(3): p. 158-164.

33. Neeper, M., et al., Cloning and Expression of a Cell-Surface Receptor for Advanced Glycosylation End-Products of Proteins. Journal of Biological Chemistry, 1992. 267(21): p. 14998-15004.

34. Ohgami, N., et al., CD36 serves as a receptor for advanced glycation endproducts (AGE). Journal of Diabetes and Its Complications, 2002. 16(1): p. 56-59.

35. Vlassara, H., et al., Identification of Galectin-3 as a High-Affinity Binding-Protein for Advanced Glycation End-Products (Age) - a New Member of the Age-Receptor Complex. Molecular Medicine, 1995. 1(6): p. 634-646.

36. Dattilo, B.M., et al., The extracellular region of the receptor for advanced glycation end products is composed of two independent structural units. Biochemistry, 2007. 46(23): p. 69576970.

37. Bongarzone, S., et al., Targeting the Receptor for Advanced Glycation Endproducts (RAGE): A Medicinal Chemistry Perspective. Journal of Medicinal Chemistry, 2017. 60(17): p. 7213-7232.

38. Perusko, M., et al., Glycation of the Major Milk Allergen beta-Lactoglobulin Changes Its Allergenicity by Alterations in Cellular Uptake and Degradation. Molecular Nutrition \& Food Research, 2018. 62(17).

39. Franca, A., et al., Macrophage scavenger receptor A mediates the uptake of gold colloids by macrophages in vitro. Nanomedicine, 2011. 6(7): p. 1175-1188.

40. Chittezhath, M., et al., Molecular Profiling Reveals a Tumor-Promoting Phenotype of Monocytes and Macrophages in Human Cancer Progression. Immunity, 2014. 41(5): p. 815829.

41. Teodorowicz, M., et al., Optimized Triton X-114 assisted lipopolysaccharide (LPS) removal method reveals the immunomodulatory effect of food proteins. Plos One, 2017. 12(3). 


\section{Supplementary materials and methods}

\section{- Lipopolysaccharide (LPS) detection}

The concentration LPS in each sample was checked using the EndoZyme ${ }^{\circledR}$ Recombinant Factor C Assay (Hyglos GmbH, Bernried am Starnberger See, Germany) according to the manufacturer's instructions.

\section{Supplementary data}

Table 5.S1 LPS contamination in samples ( $\mathrm{ng} / 0.1 \mathrm{mg}$ protein)

\begin{tabular}{lccc}
\hline Treatment & Thyroglobulin & Lysozyme & BLG \\
\hline L & 0.69 & 0.43 & 0.00 \\
L-glu & 0.71 & 0.36 & 0.01 \\
L-lac & 0.89 & 0.34 & 0.02 \\
L-gos & 0.97 & 0.39 & 0.00 \\
H & 0.20 & 0.23 & 0.00 \\
H-glu & - & - & 0.00 \\
H-lac & - & 0.32 & 0.03 \\
H-gos & 0.03 & 0.17 & 0.02 \\
W & 0.90 & 0.17 & 0.00 \\
W-glu & 0.71 & 0.24 & 0.00 \\
W-lac & 0.84 & 0.30 & 0.01 \\
W-gos & 0.80 & 0.28 & 0.00 \\
EDC & 0.05 & 0.31 & 0.00 \\
native & 1.00 & 0.63 & 0.16 \\
\hline
\end{tabular}

A

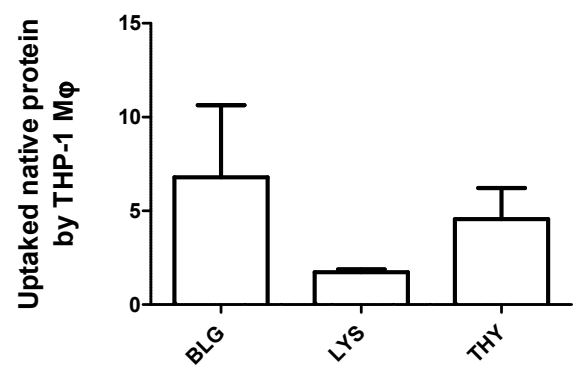

B

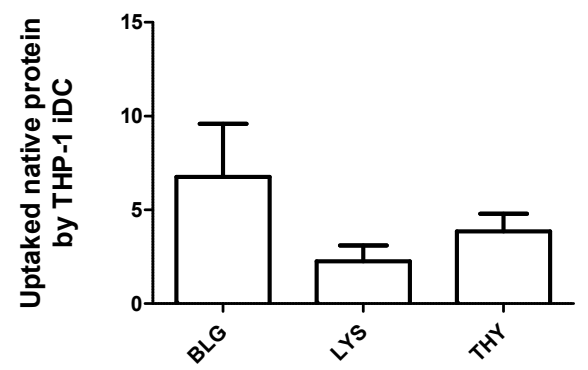

Figure 5.S1 There is no significant difference between the original uptake value of BLG, lysozyme and thyroglobulin for THP-1 derived macrophages $(M \phi, A)$ and immature dendritic cells (iDC, $B)$. The result represents the mean value $\pm S D$ of 4 independent cell experiments. No significant differences have been found using unpaired T-test with Welch's correction. 
A

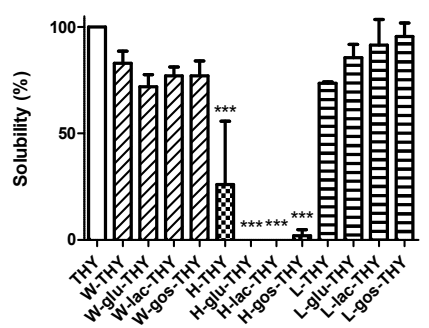

C

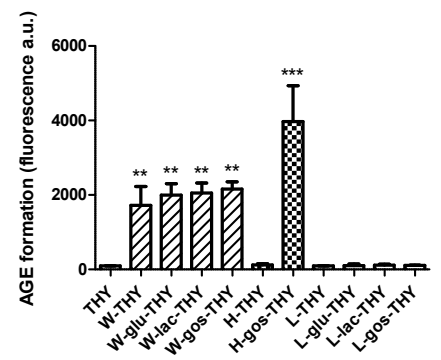

$\mathrm{E}$

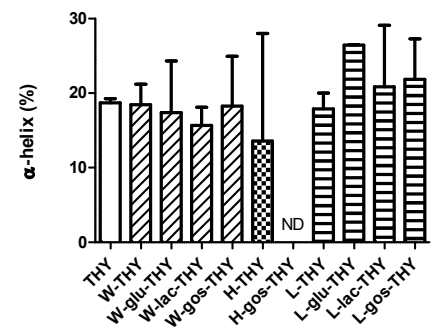

B

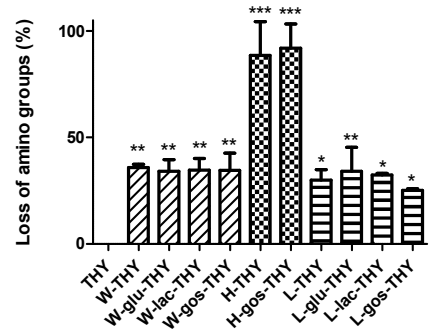

D

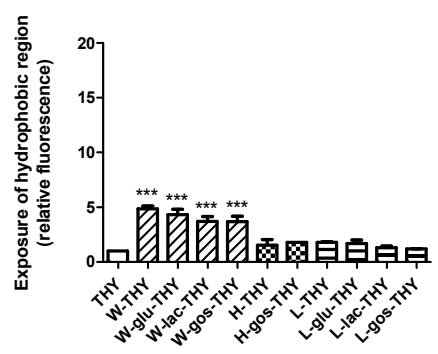

$\mathrm{F}$

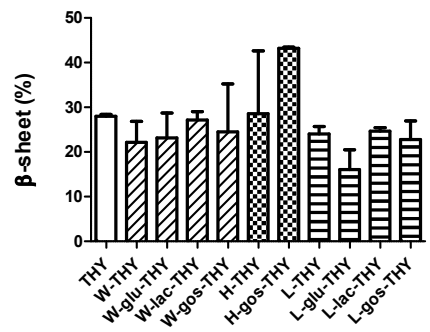

Figure 5.S2 Wet-heating and high-temperature dry-heating significantly altered the physicochemical properties of native thyroglobulin. Thyroglobulin (THY) was untreated or heated (W, $H$ or $L$ ) in the absence or presence of saccharides (glu, lac or GOS) and a number of physiochemical parameters (i.e. solubility, loss of amino group, AGE-related fluorescence, hydrophobicity, $\alpha$-helix and 6 -sheet structure) were measured. The results represent mean values $\pm S D$ of $N=4$ measurements of 2 independent experiments based on 2 independent sample sets. Statistical differences compared to native THY were calculated with Dunnett's Test: ${ }^{*} p<0.05 ;{ }^{* *} p<0.01 ;{ }^{* *} p<0.001$. ND: not detectable. 


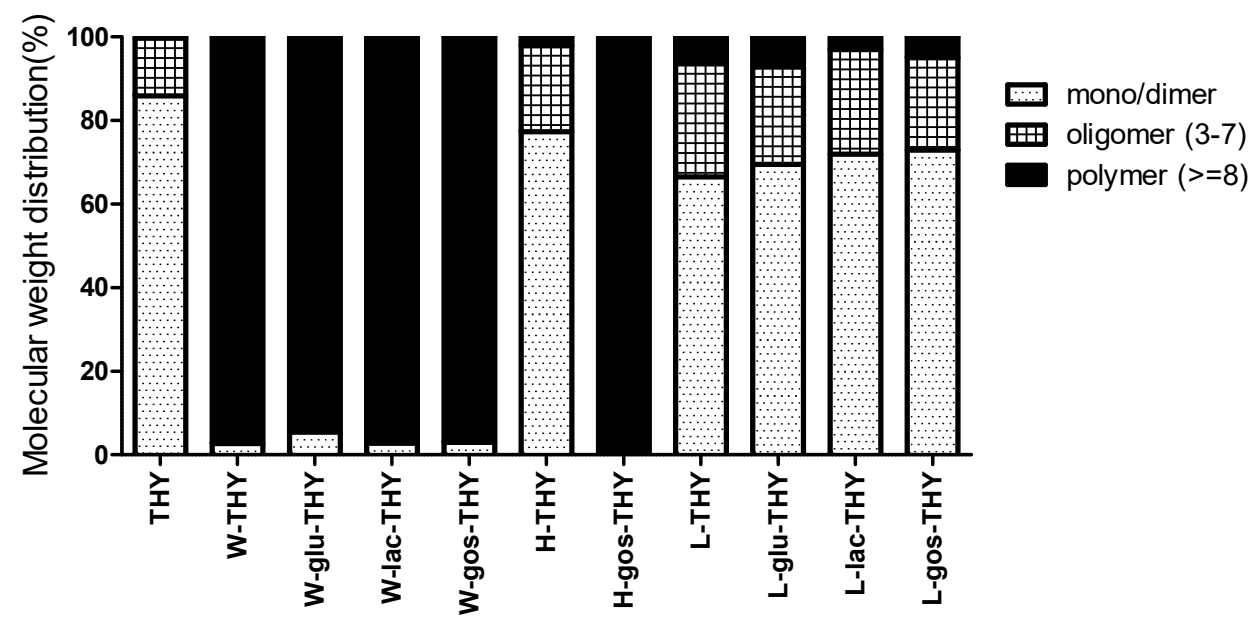

Figure 5.53 Aggregation of thyroglobulin was prominent upon wet-heating and high-temperature dryheating in the presence of GOS. Thyroglobulin (THY) was untreated or heated (W, H or L) in the absence or presence of saccharides (glu, lac or GOS) and aggregation in the soluble fraction was measured using size exclusion chromatography. The data points represent the average values of 2 independent sample sets. 
A
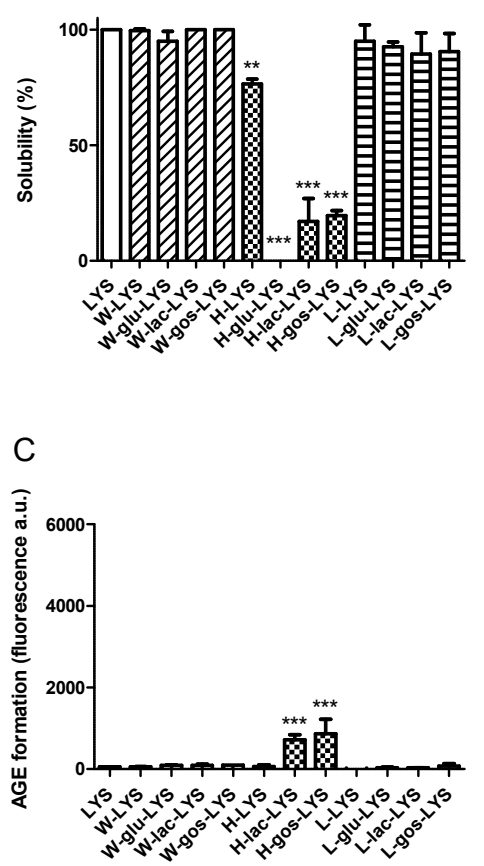

$E$

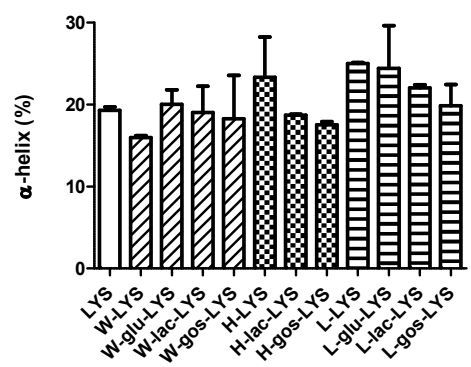

B

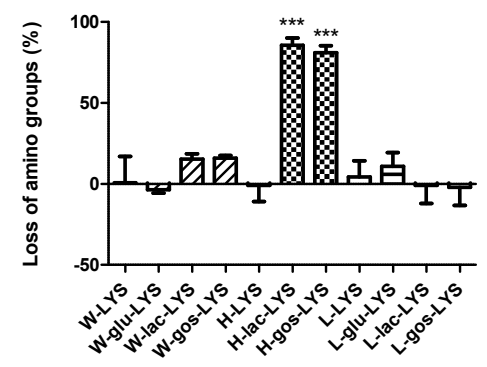

$\mathrm{D}$

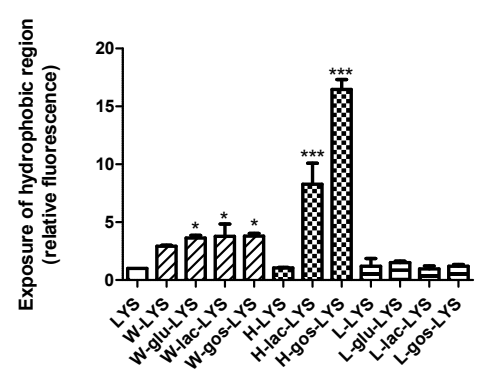

$\mathrm{F}$

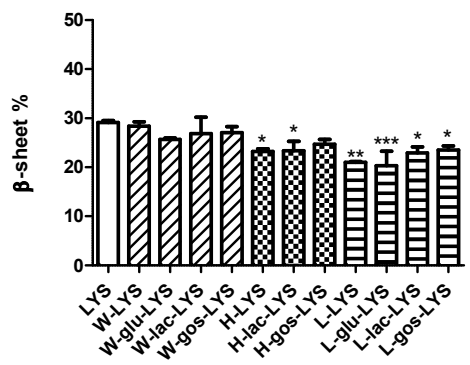

Figure 5.S4 Significant physicochemical modifications of native lysozyme were mainly induced by hightemperature dry-heating. Lysozyme (LYS) was untreated or heated (W, H or L) in the absence or presence of saccharides (glu, lac or GOS) and a number of physicochemical parameters (i.e. solubility, loss of amino group, AGE-related fluorescence, hydrophobicity, $\alpha$-helix and B-sheet structure) were measured. The results represent the mean values $\pm S D$ of $N=4$ measurements of 2 independent experiments based on 2 independent sample sets. Statistical differences compared to native LYS were calculated with Dunnett's Test: ${ }^{*} p<0.05 ;{ }^{* *} p<0.01 ;{ }^{* *} p<0.001$. 


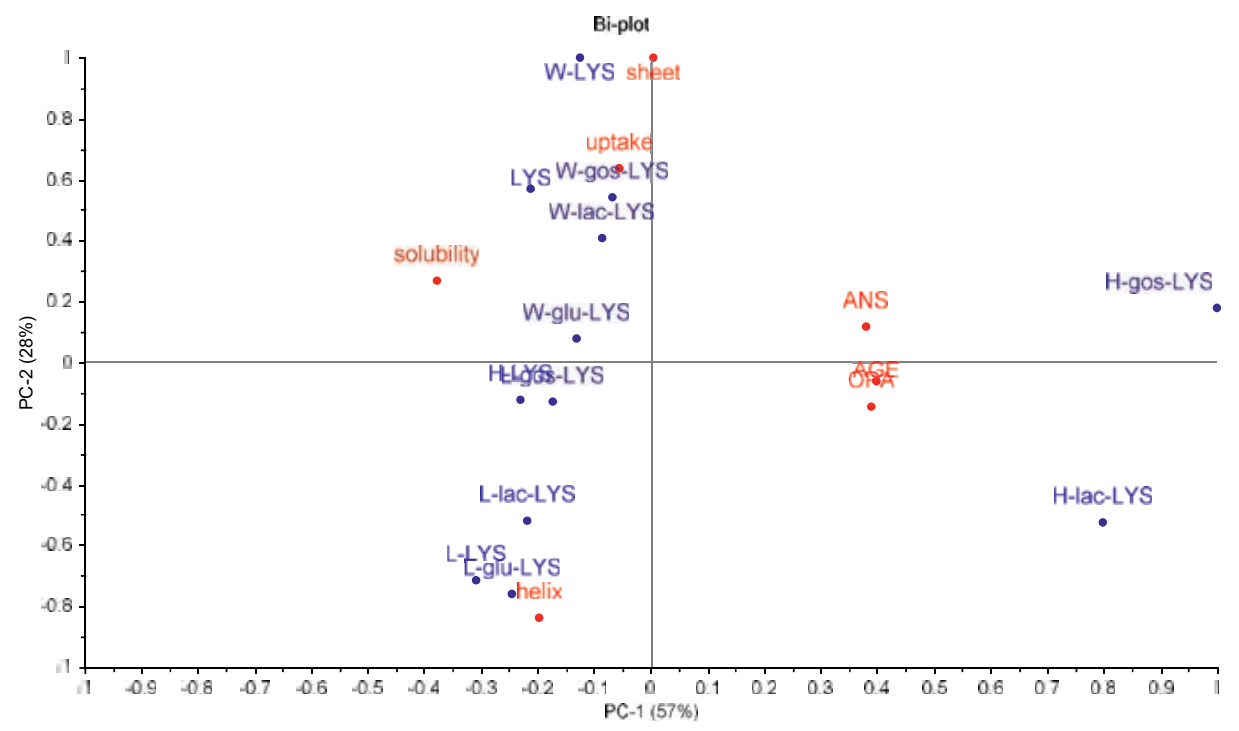

Figure 5.S5 Wet-heated lysozyme samples had physicochemical properties that are similar to native lysozyme. Lysozyme (LYS) was untreated or heated ( $W, H$ or $L$ ) in the absence or presence of saccharides (glu, lac or GOS) and tested for solubility, uptake by THP-1 macrophages (uptake), AGE formation (AGE), glycation (OPA), percentage of $\alpha$-helix (helix) or 6 -sheet (sheet), and exposure of hydrophobic regions (ANS). The aggregation-related parameters, proportion of monomer, oligomers and polymers are not shown as they did not differ between the samples. 

Chapter 6

General discussion 
The impact of nutrition on health is gaining increasing attention for a variety of reasons, for instance because of an expected increase of health care cost due to ageing populations in many countries. Only a small fraction of the foods that humans consume has not been processed, with processing nearly always including one or more heating steps. To contribute to a better understanding on the impact of processing on the biological properties of foods, this thesis studied the impact of processing-induced structural alterations of proteins on their biological properties, in particular on their digestion and on the impact on the response of cells that belong to the innate part of the immune system. As model proteins, $\beta$-lactoglobulin (BLG), lysozyme and thyroglobulin were selected, because of their differences as well as similarities in molecular mass and isoelectric point, with most focus on BLG being one of the main food allergens.

It is well known that the immunomodulatory properties of milk proteins in general, and BLG in particular, can be altered by heat processing. After pasteurization, the intestinal uptake of the whey proteins BLG and $\alpha$-lactalbumin (ALA) switched from transcellular transport to uptake via the Peyer's patches and induced significantly higher production of Th2 cytokines in a murine model [1]. The analysis of the physicochemical, cellular and molecular mechanisms behind this observation is, however, limited.

The intention of the work described in this thesis is to characterize these physicochemical, cellular and molecular mechanisms, and thereby contribute from a food technological perspective to the development of tools for estimating and minimizing the immunogenicity of processed dairy proteins. The influence of three different heating methods on the kinetics of accessibility, uptake and immune response of the proteins BLG, thyroglobulin and lysozyme by a THP-1 derived innate immune cells model was studied.

\section{Impact of processing on the physicochemical properties of protein}

During dairy processing, glycation and aggregation will occur due to the heating in the presence of a reducing saccharide (lactose or other sugars) [2,3]. Three different heating methods were applied to BLG, to achieve varying degrees and types of structural modifications (in particular aggregation, unfolding and glycation). High-temperature dry-heating $(H)$ was performed with a water activity $\left(a_{w}\right)$ of 0.59 and a temperature of $130{ }^{\circ} \mathrm{C}$, which is far above the denaturation temperature of BLG [4]. A large extent of aggregation and advanced glycation (in the presence of saccharides) was expected in this sample [5]. On the other hand, after low-temperature dry-heating $\left(L ; 50{ }^{\circ} \mathrm{C}, \mathrm{a}_{\mathrm{w}} 0.65\right)$, limited unfolding and aggregation as the processing temperature was below the denaturation temperature, but still glycation in the presence of reducing saccharides was expected [6]. Wet-heating (W) of protein 
in the presence of reducing saccharides in solution at $60{ }^{\circ} \mathrm{C}$ for $72 \mathrm{~h}$ is a method reported to induce glycation combined with denaturation and aggregation [7, 8]. A number of physicochemical measurements was performed on these potentially glycated and/or aggregated BLG samples.

\section{Effect of processing in the absence of saccharides on BLG}

As described in Chapter 3, different structural modifications were observed after these three heating methods. The wet-heating method strongly altered the structure of BLG with significant increases in molecular weight and hydrophobicity together with changes of the secondary structure. Hightemperature dry-heating led to more limited modification of the structure while low-temperature dryheating modified the protein's native structure even less.

BLG is a protein with one $\alpha$-helix and nine anti-parallel $\beta$-strands around a hydrophobic core $[9,10]$. The temperature of wet-heating induces BLG to adopt an intermediate molten globule state [11], which enables the protein to unfold, leading to exposure of hydrophobic areas and creating ordered cross-linked BLG probably via rearrangement of disulphide bonds. In another study, BLG aggregates were found to be disulphide-linked when BLG was heated at $65^{\circ} \mathrm{C}$ in solution for hours [12]. The role of disulphide bonds in the aggregation of the wet-heated aggregated BLG was clearly seen in Figure 6.1. The bands in a non-reducing gel indicating high molecular weight protein aggregates in the upper part of the W-BLG lane almost completely disappeared in SDS-PAGE under reducing conditions. The long incubation time (72 hours) enables extensive cross-linking via this disulphide interchange mechanism, leading to the formation of large but still soluble particles. This hypothesis corroborated the outcomes, as described in Chapter 3, of significant increases in hydrophobicity, and the large proportion of polymers observed in SEC chromatography, for wet-heated samples. Due to the limited $a_{w}$, protein unfolding proceeds to a much lesser extent in dry-heated samples [13]. High-temperature dry-heated BLG showed slightly more aggregates than low-temperature dry-heated samples in Figure 3.6, which indicated the effect of temperature on inducing aggregation. As indicated by all the physicochemical measurements in Chapter 3, there are no clear structural modifications in the lowtemperature heated sample, probably reflecting the simultaneous effects of the limitation in water activity and a heating temperature well below the denaturation temperature of BLG. This confirms that the heating temperature, either above or below the denaturation temperature of BLG, is an essential parameter for predicting the extent of aggregation. 


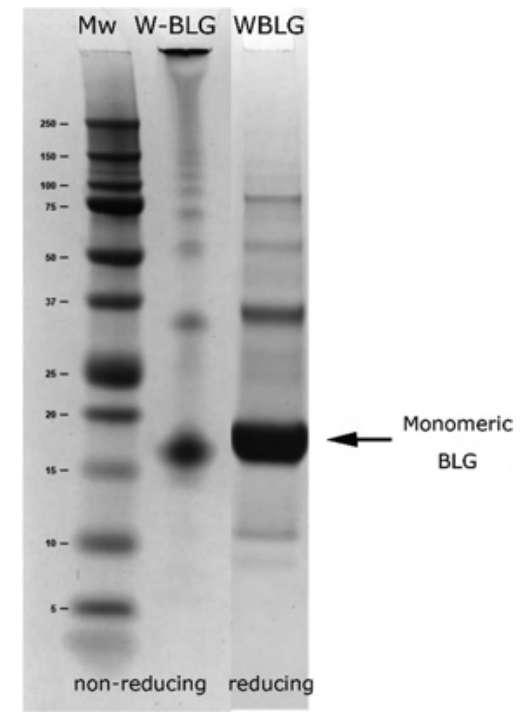

Figure 6.1 Wet-heated BLG on non-reducing SDS-PAGE showed aggregates bigger than $100 \mathrm{kDa}$, which were not visible in electrophoresis under reducing conditions. Wet-heated BLG (W-BLG) was mixed with NUPAGE ${ }^{\circledR}$ LDS Sample Buffer (4X), boiled for 5 min and loaded on $10 \%$ Bis-Tris gels with a pre-stained Precision Plus Protein ${ }^{T M}$ Dual Xtra marker for non-reducing conditions. For reducing SDS-PAGE, the protein sample was mixed with 6-mercaptoethanol (volume ratio 10:1) and further followed the same procedure.

\section{Effect of processing in the presence of reducing saccharides on BLG}

Lactose, the main saccharide present in cow's milk, is a reducing sugar that can react with free amino groups in proteins, which reaction is commonly known as the Maillard reaction [14]. To analyse the impact of this important processing-related reaction, BLG was also heated according the mentioned three processes in the presence of lactose. When dairy proteins are incorporated in complex foods, also other sugars than lactose may participate in the reaction. Therefore, also other types of reducing saccharides were included.

The presence of saccharides during heating did not introduce significant structural changes for wetheated BLG compared to heating in their absence, as shown in Figure 3.2 and 3.4-3.7. Advanced glycation end products (AGEs) are likely to be formed in the temperature range of $65-115^{\circ} \mathrm{C}$, which is higher than the temperatures used for wet-heating [15]. Indeed, there is no further loss of amino groups in the presence of saccharides, which confirmed that glycation hardly occurred. 
More severe structural changes occurred in high-temperature dry-heated BLG when saccharides were present. The reaction was severe enough to create insoluble particles, especially for monosaccharides there were significant decreases of solubility for the final products (Figure 3.S1). As shown in Figure 3.2 and 3.3, there was a significant loss of free amino groups and extensive formation of AGEs in the soluble fraction. The aggregates formed in high-temperature dry-heated BLG in the presence of saccharides were mainly insoluble and were larger than those formed in wet-heated samples. In the remaining soluble fraction, there is less disturbance of the basic native structure of BLG because changes in protein folding were limited as a consequence of the low water activity. The proportion of aggregates in the soluble fraction of high-temperature dry-heated samples was less than for wetheated samples. The presence of saccharides did not induce changes in the hydrophobicity of the soluble fraction of the samples even though the glycation reached the advanced stage and the secondary structure seemed to be altered to a larger extent compared to samples that were treated with the same method but without saccharides. The physicochemical characterisation of the samples was only performed on the soluble part.

For the low-temperature dry-heated samples, it is clear that glycation was the main reaction that occurred in the samples. The highest glycated samples were those that were treated with monosaccharides, followed by moderately glycated samples for disaccharides, and the lowest glycated samples for polysaccharides. It is known that shorter chain reducing sugars are more reactive in the Maillard reaction [16]. The inverse correlation between the chain length of the saccharides and the degree of glycation, as shown in Figure 3.2C, was as expected [17]. Despite the obvious glycation that occurred in the low-temperature dry-heated BLG samples, the limited progress to the advanced stage of the Maillard reaction can likely be explained by the low heat load. AGEs were formed limitedly (Figure 3.3) and structural changes or aggregation hardly took place in these samples (Figure 3.4-3.8).

\section{Effects of processing on lysozyme and thyroglobulin}

In Chapter 5, the same processing methods were applied to lysozyme (with a similar molecular weight but different $\mathrm{pl}$ than BLG) and thyroglobulin (much higher molecular weight but similar pl than BLG). Thyroglobulin showed physicochemical changes similar to BLG, with wet-heated samples forming a vast amount of aggregates and exhibiting a higher exposure of hydrophobic regions. High-temperature dry-heated samples showed advanced glycation while there were no clear changes for lowtemperature dry-heated samples compared to native thyroglobulin.

On the other hand, heat processed lysozyme behaved quite differently compared to the other two proteins. Hardly any modification was observed in wet-heated and low-temperature dry-heated 
lysozyme, while advanced glycation still took place in high-temperature dry-heated samples. The differences in denaturation temperature of these three proteins might play a role in the differences of their heat-induced physicochemical behaviour. Lysozyme has the highest denaturation temperature $\left(75^{\circ} \mathrm{C}\right)$ of the tested proteins $\left(65^{\circ} \mathrm{C}\right.$ and $60^{\circ} \mathrm{C}$ for $B L G$ and thyroglobulin respectively) $[4,18,19]$, which is above the temperature of wet-heating and low-temperature dry-heating, making structural modification of lysozyme less likely following these two heating methods. The unique native structure of lysozyme, encompassing 4 disulphide bonds, enables it to behave like a "hard" protein (i.e. difficult to structurally modify) [20]. The high pl of lysozyme could also introduce a high positive charge on the surface of lysozyme at the $\mathrm{pH}$ of the experiments, which might further hinder the aggregation by electrostatic repulsion.

Overall, BLG and thyroglobulin, having a similar pl and denaturation temperature, showed similar physicochemical modification after the different processing methods. A different physicochemical modification pattern was observed for lysozyme, which might be related to its higher $\mathrm{pl}$ and denaturation temperature.

\section{Relevance of digestion for exposure of native and processed BLG to innate immune cells}

Protein would only be accessible for intestinal immune cells, and subsequently play a role for the intestinal immune system, if at least the gastric digestion is survived. In Chapter 2, the impact of processing-induced modification of BLG was analysed on its digestion and transport across an artificial epithelial layer. The in vitro digestion model in this study is modified from INFOGEST, a consensus protocol that is widely accepted by numerous labs and researchers all over the world [21]. Before its establishment in 2014, digestion protocols included variable starting protein concentrations, digestion fluid composition, enzyme concentrations, reaction time and $\mathrm{pH}$ values. Such variations, as used in different studies, introduced also large variations in the outcomes, hampering comparison of results among studies. Examples of different digestion protocols are shown in Table 6.1.

BLG itself is a relatively gastric digestion-resistant protein, due to its compact structure [22]. Based on the modified digestion model, as described in Chapter 2 (Figure 2.1 and Table 2.1), heat processing was shown to increase the gastric digestibility of BLG to a different extent depending on the heating condition. 
Table 6.1 Details for different in vitro digestion systems

\begin{tabular}{|c|c|c|c|c|}
\hline Reference & $\begin{array}{l}\text { Protein } \\
\text { concentration }\end{array}$ & $\begin{array}{l}\text { Homogenous } \\
\text { system }\end{array}$ & Gastric system & Intestinal system \\
\hline $\begin{array}{l}\text { D. Dupont et } \\
\text { al. [22] }\end{array}$ & $1 \mathrm{mg} / \mathrm{ml}$ & $\begin{array}{l}63.5 \mathrm{mM} \\
\text { Phosphatidylch } \\
\text { oline, sonication }\end{array}$ & $\begin{array}{l}0.15 \mathrm{M} \mathrm{NaCl}, \mathrm{PP} 182 \\
\mathrm{U} / \mathrm{mg} \text { protein, } 1 \mathrm{~h}, \mathrm{pH} \\
2.5\end{array}$ & $\begin{array}{l}4 \mathrm{mM} \text { bile salts, } 26.1 \mathrm{mM} \text { Bis-Tris } \\
\text { buffer, BC } 0.4 \mathrm{U} / \mathrm{mg} \text { protein, PT } 34.5 \\
\mathrm{U} / \mathrm{mg}, 0.5 \mathrm{~h}, \mathrm{pH} 6.5\end{array}$ \\
\hline $\begin{array}{l}\text { M. Corzo- } \\
\text { Martínez et } \\
\text { al. [23] }\end{array}$ & $3 \mathrm{mg} / \mathrm{ml}$ & Dissolution & $\begin{array}{l}0.15 \mathrm{M} \mathrm{NaCl}, \mathrm{PP} 165 \\
\mathrm{U} / \mathrm{mg} \text { protein, } 2 \mathrm{~h}, \mathrm{pH} \\
2.5\end{array}$ & $\begin{array}{l}0.125 \mathrm{M} \text { bile salts, } 1 \mathrm{M} \mathrm{CaCl}_{2}, 0.25 \mathrm{M} \\
\text { Bis-Tris buffer, } \\
\text { PT } 35.75 \mathrm{U} / \mathrm{mg} \text { protein, } \mathrm{BC} 0.62 \mathrm{U} / \mathrm{mg} \\
\text { protein, } 15 \mathrm{~min} \text {, pH } 6.5\end{array}$ \\
\hline $\begin{array}{l}\text { M. Minekus } \\
{[24]}\end{array}$ & $100 \mathrm{mg} / \mathrm{ml}$ & Dissolution & $\begin{array}{l}\text { Simulated gastric } \\
\text { fluid*, PP } 20 \mathrm{U} / \mathrm{mg} \\
\text { food, } 2 \mathrm{~h}, \mathrm{pH} 3\end{array}$ & $\begin{array}{l}\text { Simulated intestinal fluid*, PT } 1 \mathrm{U} / \mathrm{mg} \\
\text { food, } \mathrm{BC} 0.25 \mathrm{U} / \mathrm{mg} \text { food, } 2 \mathrm{~h}, \mathrm{pH} 7.0\end{array}$ \\
\hline $\begin{array}{l}\text { T. Singh et } \\
\text { al. [25] }\end{array}$ & $100 \mathrm{mg} / \mathrm{ml}$ & $\begin{array}{l}\text { Constant } \\
\text { agitation }\end{array}$ & $\begin{array}{l}0.034 \mathrm{M} \mathrm{NaCl}, \mathrm{PP} 100 \\
\mathrm{U} / \mathrm{mg} \text { protein, } 2 \mathrm{~h}, \mathrm{pH} \\
1.9\end{array}$ & $\begin{array}{l}12 \mathrm{mM} \text { bile salts, } 10 \mathrm{mM} \mathrm{CaCl} 2,150 \\
\mathrm{mM} \mathrm{NaCl}, 4 \mathrm{mM} \text { Tris buffer, porcine } \\
\text { pancreatin }\left(8 \times \mathrm{USP}^{*}\right), 2 \mathrm{~h}, \mathrm{pH} 6.8\end{array}$ \\
\hline $\begin{array}{l}\text { M. Sanz et } \\
\text { al. [26] }\end{array}$ & $3 \mathrm{mg} / \mathrm{ml}$ & $*$ & $\begin{array}{l}0.15 \mathrm{M} \mathrm{NaCl}, \mathrm{PP} 3300 \\
\mathrm{U} / \mathrm{mg} \text { protein, } 2 \mathrm{~h}, \mathrm{pH} \\
2.5\end{array}$ & $\begin{array}{l}0.125 \mathrm{M} \text { bile salts, } 1 \mathrm{M} \mathrm{CaCl}_{2}, 0.25 \mathrm{M} \\
\text { Bis-Tris, PP } 14300 \mathrm{U} / \mathrm{mg} \text { protein, } \mathrm{BC} \\
62 \mathrm{U} / \mathrm{mg} \text { protein, } 15 \mathrm{~min}-2 \mathrm{~h}, \mathrm{pH} 6.5\end{array}$ \\
\hline $\begin{array}{l}\text { J. Boyer et } \\
\text { al. [27] }\end{array}$ & $\begin{array}{l}1000 \mathrm{mg} / \mathrm{ml} \\
\text { (whole food) }\end{array}$ & Homogenizer & $\begin{array}{l}0.14 \mathrm{M} \mathrm{NaCl}, 0.05 \mathrm{M} \\
\mathrm{KCl}, \mathrm{PP} * 1.3 \mathrm{mg} / \mathrm{g} \text { food, } \\
0.5 \mathrm{~h}, \mathrm{pH} 2.0\end{array}$ & $\begin{array}{l}\text { Porcine bile extract } 0.55 \mathrm{mg} / \mathrm{g} \text { food, } \\
\text { PP } 0.0875 \mathrm{mg} / \mathrm{g}_{\text {food }}{ }^{*}, 1 \mathrm{~h}, \mathrm{pH} 6.5\end{array}$ \\
\hline
\end{tabular}

Note: PP: porcine pepsin; PT: porcine trypsin; BC: bovine $\alpha$-chymotrypsin; *: information incomplete

Wet-heated BLG was digested the most in the gastric digestion phase, followed by high-temperature dry-heated BLG, and the least for low-temperature dry-heated BLG, which hardly differed from native BLG. The gastric digestibility seems to correlate with the extent of structural modification of BLG after processing, as a consequence of increased exposure of cleavage sites to digestive enzymes in more unfolded proteins. The increase in hydrophobicity may also enhance the accessibility of the protein to pepsin [28], which may explain the increased gastric digestion of wet-heated BLG. Following digestion, around $1 \%$ of the fraction of the BLG samples that survived the gastric digestion was translocated across a Caco-2 monolayer, except for the wet-heated samples which showed significantly lower translocation levels compared to the other samples (Figure 2.5). The high molecular weight and high hydrophobicity of the wet-heated BLG samples might have contributed to their lower translocation.

Although it was observed that the processed BLG, especially after wet-heating, was digested strongly in the gastric phase and (if undigested) hardly passed through the epithelial layer, it does not necessarily mean that there is no added value in further studying the interaction between intestinal immune cells and processed BLG samples. Firstly, almost all or a considerable proportion of resp. native and processed BLG still is intact after gastric digestion, although the remaining undigested amount differed. Approximately $60 \%$ of wet-heated was digested after gastric digestion (Table 2.1), being the highest among all the samples. Secondly, the digestion system of infants is less mature than the adult counterpart, leading to a lower degree of protein hydrolysis due to the higher gastric $\mathrm{pH}$ and 
the lower concentrations of digestive enzymes. Significantly higher amounts of undigested protein may thus reach the intestine and interact with intestinal immune cells in infants than adults [29]. It is meaningful to study the immune effect of processed BLG since infants are a major consumers of milk protein-containing powder. Lastly, the physiological intestinal transportation system is more complicated than the cell model. In the mouse model, aggregated BLG was reported to be sampled by Peyer's Patches, whereas native BLG is taken up via epithelial uptake [30]. Moreover, dendritic cells (DCs) in the lamina propria could sample antigens by protruding the epithelial layer. The increased size, a characteristic property of wet-heated BLG, was reported to enhance the uptake of particles by DCS [31].

Although digestion influences the accessibility of differently processed BLG to immune cells, it is still meaningful to perform the further immunological analyses on undigested BLG samples, due to the presence of intact protein in the gastric digest. Differently processed protein samples, in identical concentration, were used for immunological experiments to allow a better comparison between samples.

\section{Key determinants for the uptake of heat processed protein by THP-1 APCs}

In Chapter 3, the uptake of differently processed BLG samples by THP-1 macrophages was analysed and correlated with the samples' physicochemical properties, seeking the key parameters for uptake. The experiment was extended to processed thyroglobulin and lysozyme, and dendritic-like cells, to find possibly existing 'universal' key parameters that influence the uptake of processed proteins with different properties by THP-1 derived APCs. In line with the less prominent role of DCs in phagocytosis compared to macrophages, the absolute uptake level for DC of BLG, lysozyme and thyroglobulin was smaller than for macrophages (Figure 3.1, 5.1, 5.2). Since there was a strong correlation between the uptake performance of macrophages and DCs (Figure 5.2D), the data for macrophage uptake were used to further analyse the key parameters that determine the uptake of processed samples. In Chapter 3, wet-heating of BLG was reported to enhance it uptake significantly, for which hydrophobicity and aggregation were found to be the key determinants. The set of included proteins was broadened to lysozyme and thyroglobulin with intrinsic properties differing from BLG (Chapter 5). In line with physicochemical modifications of thyroglobulin being similar to BLG after processing, wetheating also significantly increased the uptake of thyroglobulin, also being strongly correlated to hydrophobicity and aggregation. It is not possible to postulate a role of hydrophobicity and aggregation in the uptake of lysozyme after exposure to different heating conditions since there is no clear change of lysozyme's physicochemical properties and uptake by macrophages. 
With the help of EDC (a protein crosslinker), aggregates could be generated without the severe structural changes as occurring upon heat-induced unfolding and exposure of hydrophobic regions. EDC-crosslinking of BLG and thyroglobulin led to the formation of aggregates but did not increase the uptake significantly. On the other hand, EDC did not lead to the expected aggregation of lysozyme as, instead of intermolecular crosslinking, probably intramolecularly crosslinking occurred [32] which altered the protein structure and increased its hydrophobicity. A significant increase of uptake for EDCtreated lysozyme was observed, which further confirmed the importance of hydrophobicity in determining the uptake capability.

The net charge of the cell surface depends on a very complex set of variables which is still disputed [33]. If the surface of cells is positively charged, due to the abundant phosphatidylserine and ion transport channels on the cell membrane, it is likely more accessible for negative particles. BLG and thyroglobulin, which both showed increased uptake, are also both negatively charged $(\mathrm{pl}<7)$ under experimental conditions. Higher uptake has been observed for more negatively charged particles for RAW 264.7 murine macrophages and for a few mammalian cell lines including leukemic cell lines UT-7 and $K 562[34,35]$. It could also be seen from Figure $5 . S 1$ that native lysozyme has the lowest absolute uptake value compared to the other two proteins. Its positive charge at physiological pH might explain why heat-treated lysozyme was not readily taken up.

Surface characteristics including hydrophobicity and charge have been shown to be essential for uptake by macrophages [36]. Based on these observations, it could be concluded that hydrophobicity plays a more profound role than aggregation in enhancing the uptake of protein by THP-1 macrophages. Surface charge might also be involved in this process, but further validation is needed.

\section{Immune response of THP-1 derived macrophages and dendritic cells upon exposure to proteins}

As indicated in Chapter 4, the THP-1 monocyte derived macrophage-like (M0) and dendritic-like cells (iDC) to a considerable extent mimicked the properties of primary monocyte derived cells. Not only the expected morphology (Figure 4.1) and higher transcription levels of typical genes was observed (Table 4.S1 and 4.S2), but also the expression of surface markers (Figure 4.4) and their antigen processing capability (Figure 4.6) fitted the observations reported in literature. Although THP-1 monocyte derived macrophages have been widely used, dendritic cells derived from THP-1 monocytes using a cytokine cocktail of GM-CSF and IL-4 were unable to show induced expression of surface marker CD86 and HLA-DR as primary monocyte-derived DCs do [37]. Although HLA-DR expression was also not significantly induced, the stimuli (LPS and IL-4) used in this thesis were able to differentiate the THP-1 
monocyte into a dendritic-like cell type. M0 exhibited the strengths of macrophages in antigen uptake, processing and inflammatory response while $\mathrm{T}$ cell stimulation and signalling between innate and adaptive immune system seemed to be more apparent in iDC.

No significant influence of the type of saccharides on protein structure modification or uptake was observed in Chapter 3. Thus, for further immune response experiments based on APCs, BLG processed by different heating methods was used, but only with glucose, as it is one of the most widely used saccharides in the food industry. After incubation with BLG samples, the transcription response of iDC was weaker than that of MO. Although iDC treated with different BLG samples did not show many immune related genes which were significantly differentially transcribed, the cytokine secretion levels of IL-8 and IL-1 $\beta$ in native BLG and L-glu-BLG incubated iDC were significantly higher than observed for other BLG samples, as shown in Figure 4.9. The cytokine inducing effect of native BLG and L-glu-BLG could be more clearly observed for $\mathrm{MO}$ in Figure 5.8. The protein structural modification of native BLG was reported to be very limited in the L-glu-BLG sample in Chapter 3. IL-8, CCL-20 and IL-1B are all related with pro-inflammatory responses which might indicate the inflammation-inducing effect of native BLG upon incubation with APCs. The inflammation-inducing effect of native BLG is also indicated by inflammation induction-related pathways which were significantly associated with $\mathrm{MO}$ treated with BLG and L-glu-BLG as shown by pathway analysis (Figure 4.8). The presence of mild glycation products in L-glu-BLG might cause a Th2 differentiation enhancing impact on MO. A similar conclusion was drawn based on the dendritic cell experiment with glycated ovalbumin [38]. It is notable that although cell exposure to W-glu-BLG led to the highest uptake, but the impact on other analysed immune response parameters was the lowest among all the samples. Thus, the uptake efficiency in APCs of protein cannot directly be linked to transcription-inducing effects.

\section{Potential immunological impact of lipopolysaccharide (LPS) and inhibitory protocol}

LPS produced by gram-negative bacteria is very commonly present in our daily life, including our diet. Its increased translocation has been associated with several adverse health conditions, including Alzheimer's disease, chronic inflammation of the gut, diabetes, etc. [39]. LPS is used by immune cells for detection of bacteria and will induce a strong pro-inflammatory cytokine production to deal with possible bacterial invasion. The levels of LPS, being a strong immune stimulus, should be kept below threshold levels in in vitro immunological studies as it will obscure the effect of the tested sample itself.

The contamination levels of LPS in the samples used for this thesis is presented in Table 3.S1 and 5.S1. The LPS concentration for all samples was below $1 \mu \mathrm{g} / \mathrm{mL}$, the threshold which was shown to lead to a significant increase of uptake by THP-1 macrophages (Figure 3.S3). BLG samples in general had lower 
LPS levels than lysozyme and thyroglobulin, which might be due to the controlled conditions during inhouse isolation of BLG from cow's milk. For further immunological research, BLG samples were used in which processed BLG showed lower LPS levels $(2.3-7.8 \mathrm{pg} / \mathrm{mL})$ than native BLG $(155 \mathrm{pg} / \mathrm{mL})$. The lower concentration of LPS in processed samples might be due to the dialysis step in sample preparation which was meant to remove the unreacted saccharides.

About $5 \mathrm{pg} / \mathrm{mL}$ of LPS was found to exert no effect on a healthy human body [40, 41]. THP-1 monocytes were reported to be less sensitive to LPS stimulation compared to primary monocytes with lower and even no production for different cytokines [42]. On the other hand, in other immunological studies 10$100 \mathrm{ng} / \mathrm{ml}$ LPS was normally regarded to be sufficient to induce a clear and obvious response, which is 1000x higher than the LPS concentration for the sample set [43-46]. The LPS related pathway was also not enhanced in data of Chapter 4. Although L-glu-BLG has a much lower LPS level than native BLG, the immune response it induced in macrophages and dendric cells is as strong as for BLG, which further supports the notion that the observed immunological effect is mainly induced by the intrinsic immunogenicity of BLG. Thus, it was concluded that no LPS inhibitory procedure is needed for these samples.

\section{Macrophage polarization after exposure to native and processed BLG}

Activated macrophages can, at the extremes of polarisation, be classified into the pro-inflammatory cell type M1 and anti-inflammatory cell type M2 [49]. Due to their plasticity, many intermediate phenotypes may occur. The M1 phenotype produces iNOS and secretes IL-1ß, tumour necrosis factor (TNF), IL-12, IL-18 and IL-23, which all promote a Th1 response. On the contrary, the M2 phenotype is characterized by a high level of IL-10 and low level of II-12, which promotes Th2 responses and is related to cell proliferation and tissue repair [50]. M2 could be further divided into M2a, M2b and M2c and $\mathrm{M} 2 \mathrm{~d}$ according to their different gene transcription profiles and cellular functions [51]. According to the protocol of Chanput et al. [46], THP-1 monocytes were first differentiated into macrophages at resting stage (M0) by PMA, then incubated 6 hours with IFN- $\gamma(20 \mathrm{ng} / \mathrm{mL})$ and LPS $(1 \mu \mathrm{g} / \mathrm{mL})$ to induce an M1 phenotype or IL-4 (20 ng/mL) to induce an M2a phenotype. The M2b or M2c phenotypes were obtained by incubating M0 with LPS $(1 \mu \mathrm{g} / \mathrm{mL})$ and IL-1 $(10 \mathrm{ng} / \mathrm{mL})$ or IL-10 $(10 \mathrm{ng} / \mathrm{mL})$ for 6 hours, respectively $[52,53]$. To analyse which direction of macrophages polarization has been induced by the BLG samples, a qPCR analysis was conducted on the THP-1 macrophages treated with native or heated BLG and phenotype controls. Transcription levels of phenotype related genes CXCL9, arginase, CCL1, CCL5, CCL15, CCL18, CCL24, CD86, CXCL8, IL-10, IL-12, mannose receptor, TGF- $\beta$, TNF- $\alpha$ were measured by qPCR. 


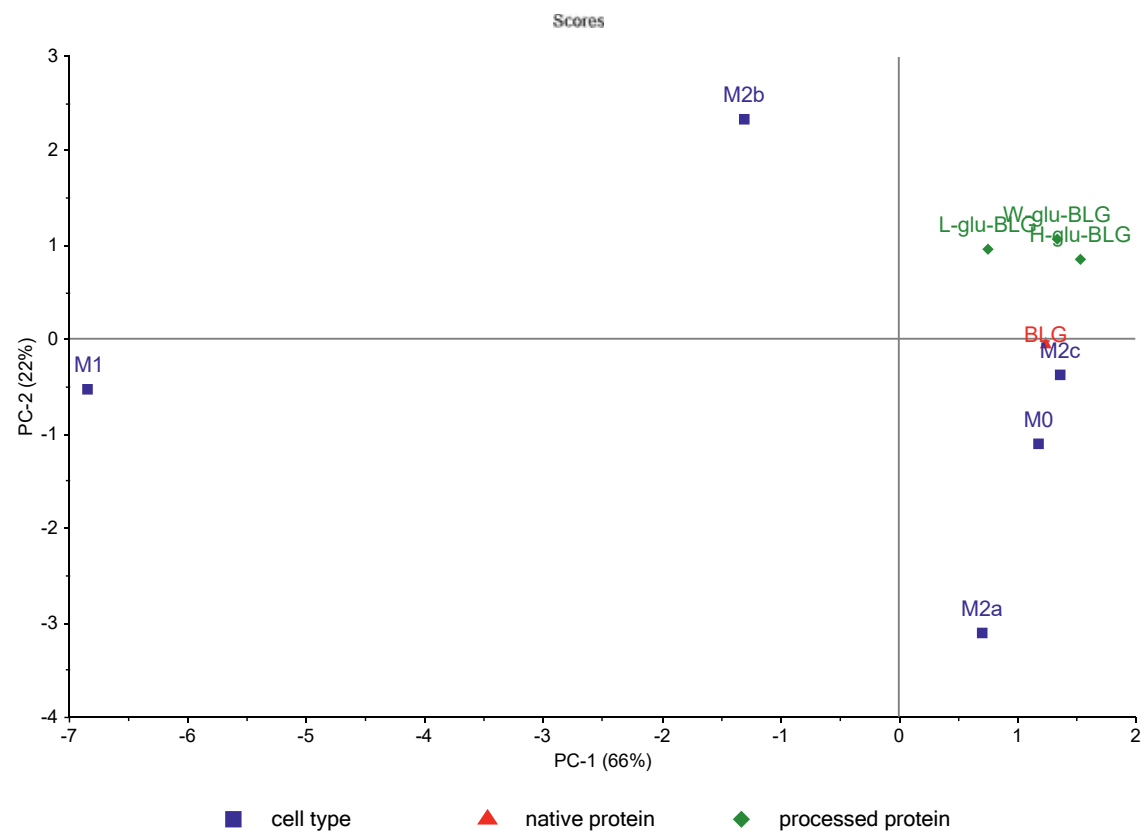

Figure 6.2 Macrophages at resting stage $(\mathrm{MO})$ treated with native BLG showed a gene transcription profile closely related to the M2c phenotype, and the ones treated with processed BLG samples related to the $M 2 b$ and $M 2 c$ phenotypes. BLG was untreated or heated $(W, L$ or $H)$ in absence or presence of glucose and tested for gene transcription of macrophage polarization markers together with $M 1, M 2 a$, M2b, M2c cell controls. Gene transcription data were plotted in a bi-plot after PCA analysis.

After incubation with BLG samples for 6 hours, macrophages treated with native BLG seemed to present a transcription profile close to $\mathrm{M} 2 \mathrm{c}$ which is related with suppression of immune responses and tissue remodelling (Figure 6.2) [53]. This may be interpreted as unexpected, as M1 is reported to be related with inflammation [54], and native BLG (pro-inflammatory) incubated MO might be stimulated into this direction. Compared to native BLG, variously processed BLG (especially lowtemperature dry-heated BLG in the presence of glucose) seemed to induce a shift towards the M2b phenotype, which is typical for its immunoregulatory functions and Th2 enhancing properties [55]. This observation corroborates the previous conclusion (Chapter 4) about the suppressive inflammatory effect of L-glu-BLG upon exposure to MO.

\section{Gene transcription profile induced by thyroglobulin and lysozyme}

The modification of physicochemical properties of thyroglobulin and lysozyme after heating and their correlation with uptake by THP-1 APCs has been studied in Chapter $\mathbf{5}$. Relatively severe modifications 
occurred in wet-heated thyroglobulin, which may be the cause of the significant increase in uptake by THP-1 APCs. Physicochemical modifications were limited in processed lysozyme and there was no clear change in uptake compared to native lysozyme. To investigate the responses of THP-1 macrophages upon exposure to heated lysozyme or thyroglobulin after uptake, the transcription levels of 21 immune related genes were measured by qPCR (Figure 6.3). There appears to be a correlation between gene transcription and physicochemical properties as the samples, in the heat map, clustered partly in relation to the heating methods. For example, all low-temperature dry-heated thyroglobulin samples were hierarchically clustered with native protein. Almost all wet-heated and high-temperature dryheated lysozyme samples were hierarchically clustered with each other. Lysozyme induced a similar gene transcription profile as thyroglobulin, with the exception of higher transcription levels for CCL1 and IL-10, to which a homeostatic or anti-inflammatory role is assigned, respectively $[58,59]$. This indicated a stronger immune modulatory and anti-inflammatory effect for lysozyme samples.

With respect to transcription of individual genes, the gene transcription level of IL-12 was suppressed for all samples treated with either protein. The strongest overall increase occurred for CXCL8 (IL-8) in macrophages that were treated with either sample. CXCL8 is a potent pro-inflammatory chemokine which is released by macrophages to recruit other cells directly after encountering antigens [56], and is produced in large amounts in response to the endotoxin LPS. The LPS-contamination in the samples can be found in Table 5.S1, which was between 0.1-1 ng/mL. This LPS concentration is unlikely to explain the macrophage response, as IL-8 production of THP-1 macrophages did not increase significantly until the LPS concentration was above $1 \mathrm{ng} / \mathrm{mL}$ (data shown in the following content). Another study showed that monocytes stimulated with $0.2 \mathrm{ng} / \mathrm{mL}$ LPS did not show clear IL-8 expression [57]. An increase was also observed for CCL18, CCL5, CCL15, LOX-1, and Galectin-3, albeit to a lesser extent than CXCL8. 
A

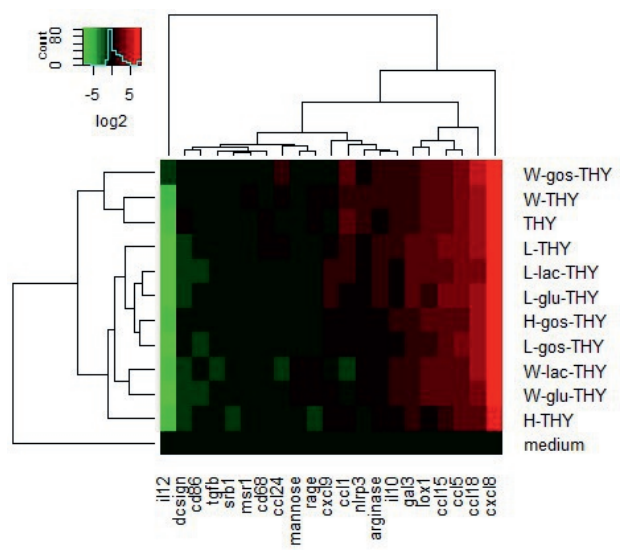

B

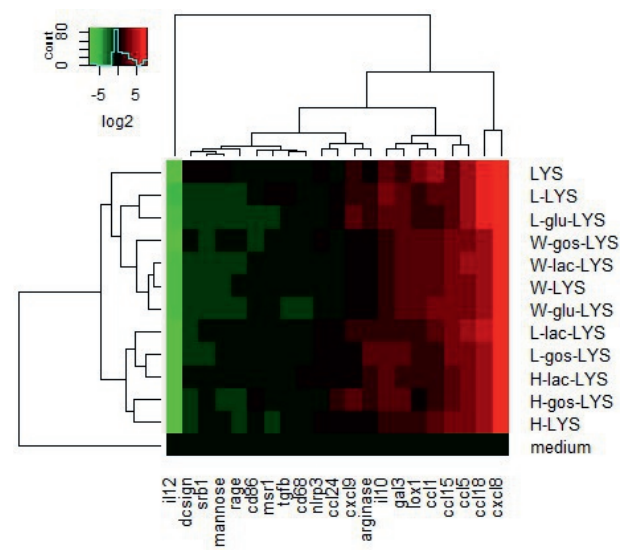

Figure 6.3 Hierarchical clustering showing different patterns of gene transcription of lysozyme and thyroglobulin samples. Thyroglobulin (THY; A) and lysozyme (LYS; B) were untreated or heated (W, H or $L$ ) in the absence or presence of saccharides (glu, lac or GOS). The plotted data was log 2 value of average fold change of $N=4$ qPCR measurements of 2 independent cell experiments based on 2 independent sample sets. The included genes are IL-12, DC-SIGN, srb1, mannose receptor, RAGE, CD86, msr1, TGF-6, CD68, NLRP3, CCL24, CXCL9, arginase, IL-10, GAL3, LOX1, CCL1, CCL15, CCL5, CCL18, CXCL8.

After exposing macrophages to the thyroglobulin and lysozyme samples, there was a high transcription level for M1 markers as CXLC8 and CCL5 [60], while the gene expression profile also showed a typical

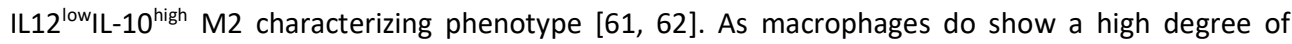
plasticity, and M1/M2 re-polarization was often observed in vivo $[63,64]$, it is plausible that the macrophages in the current study, after protein stimulation, were polarized into a unique subset, which differs from the characteristic M1 or M2 profile, similar to what was observed for a unique nondigestible polysaccharides treated macrophages phenotype [65] or a recently found M2d phenotype $[66,67]$.

To sum up, the immune responses of macrophages incubated with BLG, thyroglobulin and lysozyme seemed to be influenced by the processing methods to which the proteins had been exposed. The importance of the physicochemical modifications or uptake kinetics of protein by THP-1 macrophages for their immunological influence is not clear, whereas the initial intrinsic immunomodulatory property of the protein samples might play a dominant role. BLG showed a pro-inflammatory effect on macrophages. Lysozyme samples showed a stronger anti-inflammatory and immune modulatory effect than thyroglobulin, and the polarization of these two protein-treated macrophages does not reflect a typical M1 or M2 phenotype. These results indicate the potential immunomodulatory effect of proteins, via different processing, on macrophages. 


\section{Maturation of iDC by different concentrations of LPS}

After phagocytizing antigens, immature DCs are activated and become mature during the migration from peripheral tissue to lymph nodes, presenting the antigen on the MHC-II receptor to naïve T cells [68]. There are various protocols for maturing DCs, for which LPS is a commonly used stimulus [69]. After incubation with different concentrations of LPS for 2 days, the expression of CD209 decreased with increasing concentration, while the expression of CD83 and CD80 increased (Figure 6.4). When the concentration was above $250 \mathrm{ng} / \mathrm{mL}$, the decrease of the expression of CD209 was observed to be significant. The increase of CD83 and CD80 seemed to reach a plateau at a concentration higher than $125 \mathrm{ng} / \mathrm{mL}$. The concentration of LPS used for human primary monocyte-derived iDC maturation was normally $1 \mu \mathrm{g} / \mathrm{mL}$, and $10 \mu \mathrm{g} / \mathrm{mL}$ for mouse iDC [70-72]. This is the first time that mDCs were generated in a two-step protocol from THP-1 monocytes-derived iDC, other than the direct generation from THP1 monocytes [73]. This result demonstrated that LPS with a concentration of $500 \mathrm{ng} / \mathrm{mL}$ is sufficient to mature THP-1 derived iDC.

A

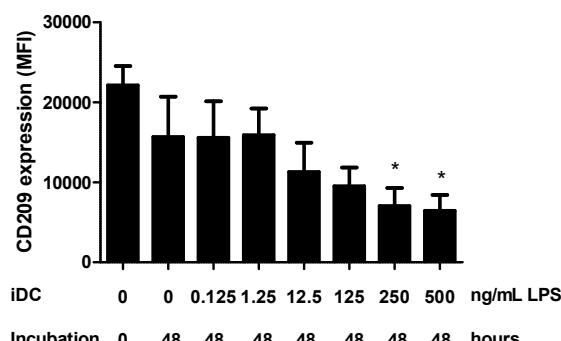

$\begin{array}{llllllllll}\text { Incubation } & 0 & 48 & 48 & 48 & 48 & 48 & 48 & 48 & \text { hours }\end{array}$

C

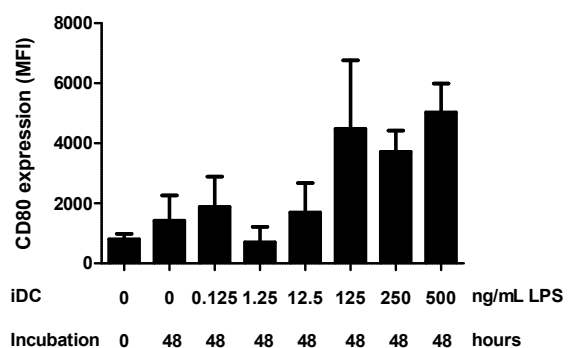

B

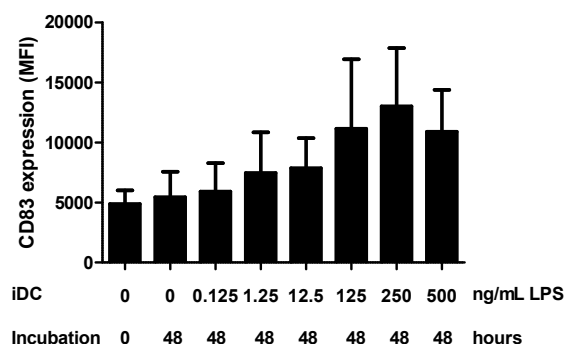

$\begin{array}{llllllllll}\text { Incubation } & 0 & 48 & 48 & 48 & 48 & 48 & 48 & 48 & \text { hours }\end{array}$

Figure 6.4 THP-1 derived iDC stimulated with $500 \mathrm{ng} / \mathrm{mL}$ LPS showed the best maturation performance. IDC was incubated with different concentration of LPS for 2 days and checked by their surface marker of CD209 (A), CD83 (B) and CD80 (C) using flow cytometry. The results represent the mean MFI $\pm S D$ of 3 independent experiments. Statistical differences compared to iDC were calculated with Dunnett's Test: ${ }^{*} p<0.05$. 
As mentioned above, there were not many significantly differentially transcribed genes in iDC incubated with BLG samples. However, the cytokine secretion levels of iDC treated with differently processed BLG showed significant differences. It would be worthwhile to investigate if differently processed BLG samples could differently mature iDC. Processed BLG in the absence of glucose was also included to further estimate the effect of glycation. No significant change of mDC markers CD209, CD83 or CD80 was observed after exposing the iDCs to heat-treated BLG-samples (Figure 6.5). The immunomodulatory capacity of BLG samples might still not be strong enough to mature iDC. A more sensitive method would then be needed to give more detailed information about the immunological influence of BLG samples. For example, a DC and CD4+ T cells coculture system is widely used to be able to capture the following T cell response after antigen-presenting of DC [38, 74]. Moreover, it gives the possibility to clearly distinguish the Th1 or Th2 skewed immune response induced by the protein samples.

A

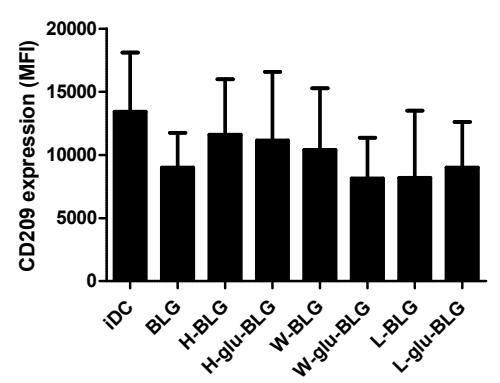

C

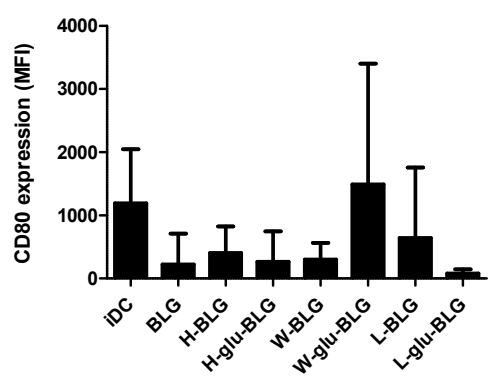

B

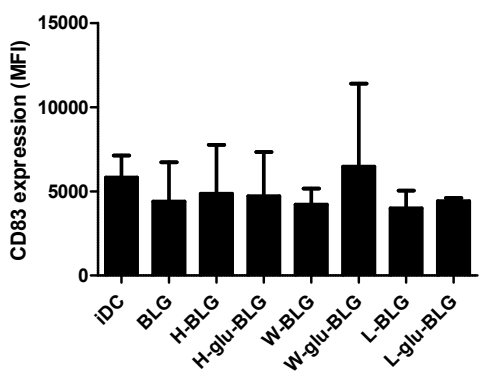

Figure 6.5 THP-1 derived IDC stimulated with different BLG samples did not lead to clear maturation. iDCs were incubated with BLG samples for 2 days and checked by their surface marker expression of CD209 (A), CD83 (B) and CD80 (C) using flow cytometry. The results represent the mean MFI $\pm S D$ of 3 independent experiments. No statistical differences compared to iDC were found. 


\section{Conclusions and future perspectives}

This thesis demonstrated that wet-heating of BLG led to a higher gastric digestibility and decreased translocation across a Caco-2 monolayer, which indicates its potentially lowered accessibility to intestinal immune cells (Chapter 2). Of all the physicochemical modifications, the hydrophobicity increase of wet-heated BLG is the key determinant for its enhanced uptake by THP-1 macrophages (Chapter 3), which also applied for other proteins with different molecular weight and pl, and THP-1 iDC as another type of APC (Chapter 5). Further analysis of the response of APCs upon exposure to processed BLG showed a strong influence of protein structural change for its immunogenicity, for macrophages in particular, which is based on a well-described and representative THP-1 monocytesderived APC model (Chapter 4). In Chapter 5, it was also observed that cytokine secretion of macrophages incubated with BLG samples did not directly correlate with its uptake behaviour.

The results of this study could help to better understand how the immunogenicity of food proteins can be modulated through processing. BLG was used as a model protein because of its prominent role in infant nutrition, and the fact that it is heated in many different ways in industry, leading to a wide range of structural and chemical modifications. Performing similar studies on other proteins, or on whole foods, will demonstrate whether, and to which extent, the observations can be extrapolated. High-temperature dry-heating is a harsh condition which leads to considerable modifications of the protein, including a major loss of solubility. The quantitative dimension of the physicochemical and immunological influence of those insoluble aggregates were not included in this thesis but remain interesting topics for further studies. Ideally, immunological experiments would be done using digested protein samples, instead of undigested protein samples, as this would better represent the situation in vivo. Firstly, bioactive peptides which play a functional role in immune modulation could also be generated upon digestion. Secondly, under in vivo conditions, gastric digestion might break down the insoluble aggregates in high-temperature dry-heated proteins, increasing its accessibility and potentially immunogenicity to immune cells. It is of utmost importance to take not only processingrelated, but also possible digestion-related effects into account when studying the physiological impact of differentially heat-treated dietary proteins. 


\section{References}

1. Roth-Walter, F., et al., Pasteurization of milk proteins promotes allergic sensitization by enhancing uptake through Peyer's patches. Allergy, 2008. 63(7): p. 882-890.

2. Geicu, O.I., et al., Proteomic and immunochemical approaches to understanding the glycation behaviour of the casein and beta-lactoglobulin fractions of flavoured drinks under UHT processing conditions. Scientific Reports, 2018. 8.

3. Puig, C., et al., Effect of processing on the composition of infant formulas. MilchwissenschaftMilk Science International, 2003. 58(9-10): p. 476-480.

4. Yan, Y.F., et al., pH-Dependent Aggregation and Disaggregation of Native beta-Lactoglobulin in Low Salt. Langmuir, 2013. 29(14): p. 4584-4593.

5. Liu, F.H., et al., Generation of Soluble Advanced Glycation End Products Receptor (sRAGE)Binding Ligands during Extensive Heat Treatment of Whey Protein/Lactose Mixtures is Dependent on Glycation and Aggregation. Journal of Agricultural and Food Chemistry, 2016. 64(33): p. 6477-6486.

6. Deng, Y., et al., Effect of Maillard induced glycation on protein hydrolysis by lysine/arginine and non-lysine/arginine specific proteases. Food Hydrocolloids, 2017. 69: p. 210-219.

7. Chevalier, F., et al., Maillard glycation of beta-lactoglobulin with several sugars: comparative study of the properties of the obtained polymers and of the substituted sites. Lait, 2001. 81(5): p. 651-666.

8. Donald, A.M., Aggregation in beta-lactoglobulin. Soft Matter, 2008. 4(6): p. 1147-1150.

9. Kuwata, K., et al., Solution structure and dynamics of bovine beta-lactoglobulin A. Protein Science, 1999. 8(11): p. 2541-2545.

10. Hamada, D., et al., High Helical Propensity of the Peptide-Fragments Derived from BetaLactoglobulin, a Predominantly Beta-Sheet Protein. Journal of Molecular Biology, 1995. 254(4): p. 737-746.

11. Qi, X.L., et al., Thermal-Denaturation of Beta-Lactoglobulin-Effect of Protein-Concentration at Ph-6.75 and Ph-8.05. Biochimica Et Biophysica Acta-Protein Structure and Molecular Enzymology, 1995. 1248(1): p. 43-49.

12. Hoffmann, M.A.M. and P.J.J.M. vanMil, Heat-induced aggregation of beta-lactoglobulin: Role of the free thiol group and disulfide bonds. Journal of Agricultural and Food Chemistry, 1997. 45(8): p. 2942-2948.

13. Cheng, B. and S.X. Cui, The Important Roles of Water in Protein Folding: an Approach by Single Molecule Force Spectroscopy. Chinese Journal of Polymer Science, 2018. 36(3): p. 379-384.

14. Jones, A.D., C.M. Tier, and J.P.G. Wilkins, Analysis of the Maillard reaction products of betalactoglobulin and lactose in skimmed milk powder by capillary electrophoresis and electrospray mass spectrometry. Journal of Chromatography A, 1998. 822(1): p. 147-154.

15. Zhu, R.G., et al., Temperature effect on formation of advanced glycation end products in infant formula milk powder. International Dairy Journal, 2018. 77: p. 1-9.

16. Chevalier, F., et al., Improvement of functional properties of beta-lactoglobulin glycated through the Maillard reaction is related to the nature of the sugar. International Dairy Journal, 2001. 11(3): p. 145-152.

17. ter Haar, R., H.A. Schols, and H. Gruppen, Effect of Saccharide Structure and Size on the Degree of Substitution and Product Dispersity of alpha-Lactalbumin Glycated via the Maillard Reaction. Journal of Agricultural and Food Chemistry, 2011. 59(17): p. 9378-9385.

18. Edelhoch, H. and H. Metzger, Properties of Thyroglobulin .5. Properties of Denatured Thyroglobulin. Journal of the American Chemical Society, 1961. 83(6): p. 1428-\&.

19. Chang, J.Y. and L. Li, The unfolding mechanism and the disulfide structures of denatured lysozyme. Febs Letters, 2002. 511(1-3): p. 73-78.

20. Arai, T. and W. Norde, The Behavior of Some Model Proteins at Solid Liquid Interfaces .1. Adsorption from Single Protein Solutions. Colloids and Surfaces, 1990. 51: p. 1-15. 
21. Brodkorb, A., et al., INFOGEST static in vitro simulation of gastrointestinal food digestion. Nature Protocols, 2019. 14(4): p. 991-1014.

22. Dupont, D., et al., Comparative resistance of food proteins to adult and infant in vitro digestion models. Molecular Nutrition \& Food Research, 2010. 54(6): p. 767-780.

23. Corzo-Martinez, M., et al., Effect of glycation on the gastrointestinal digestibility and immunoreactivity of bovine beta-lactoglobulin. International Dairy Journal, 2010. 20(11): p. 742-752.

24. Minekus, M., et al., A standardised static in vitro digestion method suitable for food - an international consensus. Food \& Function, 2014. 5(6): p. 1113-1124.

25. Singh, T.K., et al., Influence of heat and shear induced protein aggregation on the in vitro digestion rate of whey proteins. Food \& Function, 2014. 5(11): p. 2686-2698.

26. Sanz, M.L., et al., Characterization and in vitro digestibility of bovine beta-lactoglobulin glycated with galactooligosaccharides. Journal of Agricultural and Food Chemistry, 2007. 55(19): p. 7916-7925.

27. Boyer, J., D. Brown, and R.H. Liu, In vitro digestion and lactase treatment influence uptake of quercetin and quercetin glucoside by the Caco-2 cell monolayer. Nutrition Journal, 2005. 4.

28. Tang, J., Specificity of Pepsin and Its Dependence on a Possible Hydrophobic Binding Site. Nature, 1963. 199(489): p. 1094-\&.

29. Perdijk, O., et al., Cow's Milk and immune Function in the Respiratory Tract: Potential Mechanisms. Frontiers in Immunology, 2018. 9.

30. Stojadinovic, M., et al., Cross-Linking of beta-Lactoglobulin Enhances Allergic Sensitization Through Changes in Cellular Uptake and Processing. Toxicological Sciences, 2014. 140(1): p. 224-235.

31. Seydoux, E., et al., Size-dependent accumulation of particles in lysosomes modulates dendritic cell function through impaired antigen degradation. International Journal of Nanomedicine, 2014. 9: p. 3885-3902.

32. Yamada, H., et al., Intramolecular Cross-Linkage of Lysozyme - Imidazole Catalysis of the Formation of the Cross-Link between Lysine-13 (Epsilon-Amino) and Leucine-129 (AlphaCarboxyl) by Carbodiimide Reaction. Biochemistry, 1983. 22(19): p. 4551-4556.

33. Chen, B.D., et al., Targeting Negative Surface Charges of Cancer Cells by Multifunctional Nanoprobes. Theranostics, 2016. 6(11): p. 1887-1898.

34. Xiao, K., et al., The effect of surface charge on in vivo biodistribution of PEG-oligocholic acid based micellar nanoparticles. Biomaterials, 2011. 32(13): p. 3435-3446.

35. Yue, Z.G., et al., Surface Charge Affects Cellular Uptake and Intracellular Trafficking of Chitosan-Based Nanoparticles. Biomacromolecules, 2011. 12(7): p. 2440-2446.

36. Frohlich, E., The role of surface charge in cellular uptake and cytotoxicity of medical nanoparticles. International Journal of Nanomedicine, 2012. 7: p. 5577-5591.

37. Larsson, K., M. Lindstedt, and C.A.K. Borrebaeck, Functional and transcriptional profiling of MUTZ-3, a myeloid cell line acting as a model for dendritic cells. Immunology, 2006. 117(2): p. 156-166.

38. Hilmenyuk, T., et al., Effects of glycation of the model food allergen ovalbumin on antigen uptake and presentation by human dendritic cells. Immunology, 2010. 129(3): p. 437-445.

39. Wassenaar, T.M. and K. Zimmermann, Lipopolysaccharides in Food, Food Supplements, and Probiotics: Should We be Worried? European Journal of Microbiology and Immunology, 2018. 8(3): p. 63-69.

40. Salden, H.J.M. and B.M. Bas, Endotoxin Binding to Platelets in Blood from Patients with a Sepsis Syndrome. Clinical Chemistry, 1994. 40(8): p. 1575-1579.

41. Guo, Y.M., et al., Serum Levels of Lipopolysaccharide and 1,3-beta-D-Glucan Refer to the Severity in Patients with Crohn's Disease. Mediators of Inflammation, 2015.

42. Schildberger, A., et al., Monocytes, Peripheral Blood Mononuclear Cells, and THP-1 Cells Exhibit Different Cytokine Expression Patterns following Stimulation with Lipopolysaccharide. Mediators of Inflammation, 2013. 
43. Bosshart, H. and M. Heinzelmann, THP-1 cells as a model for human monocytes. Annals of Translational Medicine, 2016. 4(21).

44. Bjorkbacka, H., et al., The induction of macrophage gene expression by LPS predominantly utilizes Myd88-dindependent signaling cascades. Physiological Genomics, 2004. 19(3): p. 319330.

45. Tedesco, S., et al., Convenience versus Biological Significance: Are PMA-Differentiated THP-1 Cells a Reliable Substitute for Blood-Derived Macrophages When Studying in Vitro Polarization? Frontiers in Pharmacology, 2018. 9.

46. Chanput, W., et al., Characterization of polarized THP-1 macrophages and polarizing ability of LPS and food compounds. Food \& Function, 2013. 4(2): p. 266-276.

47. Teodorowicz, M., et al., Optimized Triton X-114 assisted lipopolysaccharide (LPS) removal method reveals the immunomodulatory effect of food proteins. Plos One, 2017. 12(3).

48. Jurga, A.M., et al., Lipopolysaccharide from Rhodobacter sphaeroides (TLR4 antagonist) attenuates hypersensitivity and modulates nociceptive factors. Pharmaceutical Biology, 2018. 56(1): p. 275-286.

49. Isidro, R.A. and C.B. Appleyard, Colonic macrophage polarization in homeostasis, inflammation, and cancer. American Journal of Physiology-Gastrointestinal and Liver Physiology, 2016. 311(1): p. G59-G73.

50. Orecchioni, M., et al., Macrophage Polarization: Different Gene Signatures in M1(LPS+) vs. Classically and M2(LPS-) vs. Alternatively Activated Macrophages. Frontiers in Immunology, 2019. 10.

51. Mantovani, A., et al., The chemokine system in diverse forms of macrophage activation and polarization. Trends in Immunology, 2004. 25(12): p. 677-686.

52. Martinez, F.O., et al., Macrophage activation and polarization. Frontiers in BioscienceLandmark, 2008. 13: p. 453-461.

53. Lurier, E.B., et al., Transcriptome analysis of IL-10-stimulated (M2C) macrophages by nextgeneration sequencing. Immunobiology, 2017. 222(7): p. 847-856.

54. Ley, K., M1 Means Kill; M2 Means Heal. Journal of Immunology, 2017. 199(7): p. 2191-2193.

55. Wang, L.X., et al., M2b macrophage polarization and its roles in diseases. Journal of Leukocyte Biology, 2019. 106(2): p. 345-358.

56. Klezovitch, O., C. Edelstein, and A.M. Scanu, Stimulation of interleukin-8 production in human THP-1 macrophages by apolipoprotein(a) Evidence for a critical involvement of elements in its C-terminal domain. Journal of Biological Chemistry, 2001. 276(50): p. 46864-46869.

57. Monguio-Tortajada, M., et al., Low doses of LPS exacerbate the inflammatory response and trigger death on TLR3-primed human monocytes. European Journal of Clinical Investigation, 2018. 48: p. 74-74.

58. Iyer, S.S. and G. Cheng, Role of interleukin 10 transcriptional regulation in inflammation and autoimmune disease. Crit Rev Immunol, 2012. 32(1): p. 23-63.

59. Esche, C., C. Stellato, and L.A. Beck, Chemokines: Key players in innate and adaptive immunity. Journal of Investigative Dermatology, 2005. 125(4): p. 615-628.

60. Xuan, W.J., et al., The chemotaxis of $M 1$ and $M 2$ macrophages is regulated by different chemokines. Journal of Leukocyte Biology, 2015. 97(1): p. 61-69.

61. Liu, C.Y., et al., M2-polarized tumor-associated macrophages promoted epithelialmesenchymal transition in pancreatic cancer cells, partially through TLR4/IL-10 signaling pathway. Laboratory Investigation, 2013. 93(7): p. 844-854.

62. $\mathrm{Li}, \mathrm{Y}$, et al., Pleiotropic regulation of macrophage polarization and tumorigenesis by formyl peptide receptor-2 (vol 30, pg 3887, 2011). Oncogene, 2011. 30(42): p. 4373-4374.

63. Shapouri-Moghaddam, A., et al., Macrophage plasticity, polarization, and function in health and disease. Journal of Cellular Physiology, 2018. 233(9): p. 6425-6440.

64. Atri, C., F.Z. Guerfali, and D. Laouini, Role of Human Macrophage Polarization in Inflammation during Infectious Diseases. International Journal of Molecular Sciences, 2018. 19(6). 
65. Tang, Y.F., et al., Macrophages treated with non-digestible polysaccharides reveal a transcriptionally unique phenotype. Journal of Functional Foods, 2017. 36: p. 280-289.

66. Wang, Q.S., et al., Fra-1 protooncogene regulates IL-6 expression in macrophages and promotes the generation of M2d macrophages. Cell Research, 2010. 20(6): p. 701-712.

67. Duluc, D., et al., Tumor-associated leukemia inhibitory factor and IL-6 skew monocyte differentiation into tumor-associated macrophage-like cells. Blood, 2007. 110(13): p. 43194330.

68. Mbongue, J.C., et al., The Role of Dendritic Cell Maturation in the induction of insulinDependent Diabetes Mellitus. Frontiers in Immunology, 2017. 8.

69. Zhou, F., G.X. Zhang, and A. Rostami, Distinct Role of IL-27 in Immature and LPS-Induced Mature Dendritic Cell-Mediated Development of CD4(+)CD127(+)3G11(+) Regulatory T Cell Subset. Frontiers in Immunology, 2018. 9.

70. Kawamura, K., et al., Differentiation, maturation, and survival of dendritic cells by osteopontin regulation. Clinical and Diagnostic Laboratory Immunology, 2005. 12(1): p. 206-212.

71. Granucci, F., et al., Early events in dendritic cell maturation induced by LPS. Microbes and Infection, 1999. 1(13): p. 1079-1084.

72. Wang, Y.C., et al., Lipopolysaccharide-induced Maturation of Bone Marrow-derived Dendritic Cells Is Regulated by Notch Signaling through the Up-regulation of CXCR4. Journal of Biological Chemistry, 2009. 284(23): p. 15993-16003.

73. Berges, C., et al., A cell line model for the differentiation of human dendritic cells. Biochemical and Biophysical Research Communications, 2005. 333(3): p. 896-907.

74. Ge, Q., et al., Homeostatic $T$ cell proliferation in a $T$ cell-dendritic cell coculture system. Proceedings of the National Academy of Sciences of the United States of America, 2002. 99(5): p. 2983-2988. 

Summary 
Heat processing is a common procedure in producing cow's milk products in industry, amongst others to extend their shelf life. Thermal processing is reported to influence a protein's structure and potentially its immunogenicity. This thesis describes the impact of various types of processing on structural characteristics of $\beta$-lactoglobulin (BLG), as the major allergen in cow's milk allergy, and on the immune response of antigen presenting cells (APCs) in particular. These cells were chosen as they are the first immune cells to come in contact with the exogenous food protein and subsequently initiate further immune responses, including the sensitization step of the allergic reaction. Next to BLG, thyroglobulin and lysozyme were studied in the thesis to estimate whether generally applicable conclusions could be drawn on the relationship between structural modifications of proteins and the subsequent immune responses.

This thesis studied a comprehensive set of parameters that were considered to be relevant for this question, from a protein's accessibility to intestinal APCs after processing, its physicochemical structural modifications to its uptake by APCs and further immune responses. To generate a range of structural modifications, including unfolding, aggregation and glycation, three different heating methods were used. Reducing saccharides were also included in the heating process to induce glycation, which quite commonly occurs in milk processing and is expected to be important for structural modifications and immunogenicity of the protein. The methods were wet-heating (W; heating in solution at $60^{\circ} \mathrm{C}$ for 3 days) which led to aggregation of protein, high-temperature dryheating $\left(\mathrm{H}\right.$; heating at $130^{\circ} \mathrm{C}$ for 10 minutes) for advanced glycation and low-temperature dry-heating (L; heating at $50{ }^{\circ} \mathrm{C}$ for 9 hours) for mild glycation.

In Chapter 2, the changes of the accessibility of BLG to intestinal immune cells after different processing methods was studied. The intestine is the main surface where exogenous proteins would interact with the immune system, thus the digestion and transport of proteins across the intestinal epithelial cell layer may be important for its accessibility to intestinal immune cells. A modified version of the current consensus in vitro digestion INFOGEST protocol and a Caco-2 insert transwell system, which mimicked the intestinal epithelial monolayer, were used. The gastric digestibility increased significantly when the protein structure was severe modified (as observed for wet-heated BLG), being specifically enhanced by the exposure of hydrophobic regions, while the influence from the presence of lactose during processing was limited. Translocation studies of BLG across Caco-2 cell monolayers showed a lower translocation rate of wet-heated BLG compared to the otherwise processed BLGsamples. Our study indicates that structural modifications of BLG after heating (especially wet-heating) enhance its digestibility, in particular gastric digestibility, and reduce the translocation efficiency across intestinal epithelial cells. Of all the heat treatment methods, the wet-heating method decreased the accessibility of BLG to intestinal APCs the strongest. 
After the gastric digestion and being translocated through the epithelial layer, protein uptake by APCS would be the first step for its interaction with immune cells. In Chapter 3, macrophages derived from THP-1 monocytes were exposed to fluorescently labelled BLG samples and the uptake was calculated after measuring the intracellular fluorescent signal. Wet-heated BLG showed significantly higher uptake by macrophages compared to native and otherwise processed BLG samples. The presence of saccharides during processing, and hence the resulting glycation, was found to be not relevant for uptake efficiency. To link its strong uptake with the structural properties of processed BLG, a set of physicochemical parameters including molecular weight, hydrophobicity, amyloid-like structure, degree of glycation, surface charge, and secondary structure was measured. Statistical analysis demonstrated that uptake was correlated strongly to hydrophobicity, amyloid-like structures, and aggregation of the protein. Due to the fact that both amyloid-like structures were reported to be related to the increase of hydrophobicity, we postulate that the exposure of hydrophobic regions and aggregation are the leading physicochemical characteristics to explain the significantly higher uptake of wet heated BLG samples by THP-1 macrophages.

In Chapter 4, the immune response of APCs upon exposure to differently processed BLG was measured by both gene transcription and expression levels. In addition to macrophages, whose role is mainly in antigen phagocytosis, dendritic cells as another type of APCs that play an important role in signaling between the innate and adaptive immune system were also included in our study. A biological response is determined by the actor (in the case of this thesis processed protein samples) and the recipient (in this thesis the innate immune cells). To be able to obtain a clearer picture of the importance of variation in the structure of the processed proteins, a read-out system with a maximally homogenous genetic background was developed. For these experiments, macrophages and dendritic cells were both derived from THP-1 monocytes. The differences between macrophages and dendritic cells were clearly shown by their different morphology, transcription (microarray), and protein expression levels, which is in line with their specific functions in inducing an inflammatory response and $T$ cell signaling, respectively. After incubation with native and differently heat treated BLG, stronger transcriptional responses were found in macrophages than dendritic cells. For macrophages specifically, native and low-temperature dry-heated BLG showed stronger transcription-modulating effects, while there was not a clear impact from the otherwise treated BLG samples. This might be due to the strong structural modifications of BLG in these other samples. (Limited) glycation, as occurring in low-temperature dry-heated BLG, might be related to its T helper 2 (anti-inflammatory) inducing property. For immature dendritic cells, hardly any transcriptional response to processing-modified BLG was observed. Our results thus indicate that processing-induced structural modification influence the transcriptional, and in particular the immune response, of macrophages. 
Chapter 3 showed that the uptake of BLG by THP-1 macrophages differed after different processing methods and was positively correlated with hydrophobicity and aggregation. In Chapter 5, we applied the same three processing methods that were applied to BLG also to lysozyme and thyroglobulin, which have different pl or molecular weight compared to BLG, respectively. Uptake was tested in both THP-1 monocytes-derived macrophages and dendritic cells. A strong correlation between the uptake of the different protein samples by macrophages and dendritic cells was found and the former cells showed stronger uptake capability than the latter ones. The key role of hydrophobicity, over aggregation, in determining the uptake was confirmed by the results for lysozyme and thyroglobulin and the chemically crosslinked form of all three proteins. Several uptake routes were shown to contribute to the uptake of BLG by macrophages. However, cytokine responses following exposure of macrophages to BLG samples were not related to the levels of uptake. Together, our results demonstrate that heat-treatment-induced increased hydrophobicity is the prime driving factor in uptake, but also shows that the further immune response by APCs might be governed by other parameters than uptake capability.

In conclusion, this thesis showed that physicochemical modifications of proteins, e.g. exposure of hydrophobic regions as a consequence of the wet-heating processing, influences its accessibility and uptake by APCs stronger than the other two heating methods. Moreover, the potential inherent proinflammatory effect of BLG on macrophages was reduced by severe structural changes. These outcomes may contribute to reduce the incidence and prevalence of CMA, and possible adverse immune effects from milk proteins, by better understanding of the underlying immunological mechanisms. 
Acknowledgements 
$\mathrm{PhD}$ is never always an easy and joyful experience, at least for most of the candidates. What exactly supported me to pass through these 4 years and 9 months is difficult to conclude. From simply attempting to get the highest education degree in the beginning, to prove myself and enjoying the feeling of successful experiments during the procedure, to of course feeling lost, depressed and cannot wait to finish. After all the ups and downs, noticing the fact that the graduation is right around the corner, I became more and more reluctant to face the end. This thesis was a wonderful experience that I could not have finished without all of you in my life.

I would first like to express my appreciation to Harry Wichers-my dear promoter, supervisor and big boss. My PhD life literately starts from the day you picked me from Ede-Wageningen station, followed by a warm welcome. You have been always supportive and optimistic of my work, which dilutes the occasional occurred stress and sorrow during the project. Your passion for scientific work stimulated me a lot, I hope to be a great scholar of wide knowledge like you one day. Thanks for never giving me up on all the problems. Thanks for your encouragement and cheerful words helping me through those embarrassing moments. Sorry for being stubborn and "rude" sometimes during the discussions, you know I did not mean it. I still need to learn the communication skills from you, and I will miss your humour and especially the jokes to kick-off our Tuesday morning meetings.

Kasper, thanks for being my co-promotor. You are famous for the quick response and being very clearminded during the discussions among the students. And I must repeat it here again, as it is the truth. The work with you has been so effective which is something I enjoyed a lot. The suggestions and advice are always straight to the point, making them clear and relatively easy to handle. Your broad knowledge in related fields keeps amazed me and your memory is always right no matter how strongly I wanted to challenge secretly. I really appreciated to have you in the supervision team and your judging issues from a "realist" perspective sometimes.

Coen, as the other co-promotor, I admire you as a pure scientist. It is really lucky to have you help me find another angle to think about the data and improve it to a much higher level. That is the sprite of the scientist, never stop the desire to be better which I still need to learn. Thank you for your supervision and for sharing all the experimental and statistical tricks. Your positive attitude really helped me to pass through those "bad" experiment days. You are also remarkable for having an eye for the "ignored corner", care for everyone, and brave to be the atmosphere regulator which I also learned from you.

And of course, I will never forget the support and help from you both in the lab and in daily life, dear Shanna. I learned the rigorous attitude to experiment from you and Marit. Good luck with finalizing your PhD thesis. And a bunch of thanks to all the ladies in the lab for helping me no matter when I needed, Dianne, Monic, Renata and Els. Nicole, thanks for your inspiring talk and encouragement. Diederik and Jurriaan, your cheerful research altitude has influenced me, and thank you for the intentionally $\mathrm{PhD}$ (food) feeding sometimes. Annelies and Marion, thanks for arranging so many meaningful activities and supporting all the administration issues. 
Liyou, Priscilla, Francisca, Isabelle,Yongfu, Baart, Tamara and Yusi, it is great to share the office with your guys. Having the chance to chat or sometimes complain about work and life with you has been the most relaxing moment of the day. Thanks for all my thesis students, Nina, leva, Ilaria, Yankang and Ellen for your hard work. The result has contributed a lot to my thesis, and I could not finish it without you. I have learned a lot from you as well. Wish you all the best for the future no matter if you decided to continue in academia or in industry.

Hannah, thanks for your comfort and the discussions about the topic, nice to have you together with me for our Tuesday early meetings. Gosia, Arifa and Hub, thanks for involving me in your immunology meeting and for supporting me with the inhibition ELISA experiment. All the people from Food Chemistry, Jean-Paul, Henk, Marie, Peter, Sjors, Madelon, Silvia, Roy, Rene, Yuxi, Dazhi, Jolanda, and the others, thanks for including me in your presentations and social activities. I really like the meaningful discussion, the interesting PhD trips, and the generous laboratory help from you.

There is another group of people, in Helix, I want to especially thank. Klaske, thanks for involving me in your project and trusting me in handling some tasks independently. The other Danny-Deng Lei, Susanne, Xiaolin, Miranda and Mieke and Shohreh, you are the best teammates and officemates ever. My last half-year as a research assistant would not gone so well without you around.

Dianna, Ronald and lovely Mia Jane, I will always remember the dinner and parties we had together in Wageningen and Amsterdam. Thanks for hosting, offering great accompany, and the intention to award me with another PhD diploma to be coffee free. Michèle Delly from far far away Switzerland, you are one of the best achievements I gained from the internship, a real friend. Thanks for exchanging the Christmas gifts and postcards with me. Let's keep doing it no matter where we will be. Marie Declerfayt, I am proud to have the chance to work with an artist, achieving masterpieces related to my project. Your strong interest and extraordinary understanding of my project keeps surprising me. Lucas, Shraddha, Patrick, and the others from Corridor 5B3 and the House OB1, I regarded you as half-family for my living in the Netherlands. It is a great pleasure to enjoy all the amazing gatherings with you. Lima, Marko, Marcel, Shahin, Alberto, Klazina and all the other former members of SBWW, thank you for being there every Tuesday night busy fixing bikes with me. I enjoyed the cosy dinners and the relaxing chats afterwards as much as the workshops. 谷方婕, 侯家鹏和奥利奥一家子, 王溪, 王啨珏 (小双), 感谢有你们的陪伴, 希望你们在荷兰过的越来越好, 生活美满, 家庭幸福!

Aart Wijnstekers, the brain trust, top chef and proofreader of this booklet, a certain part of the thesis belongs to you. No matter what life brings us, hope we always remember this period. Thank you for being there for me. And also thanks to Ernie, Mieke and Rosa, for your warm Sinterklaas gifts with English poets. Your reading vibe strongly inspired and influenced me. PS: I really like to be called Yingying by you. Groenje, Stockje and Witje, there is a saying that a relationship starts from the moment you name the item. I will miss you a lot.

最后的最后, 我想对各位亲友说, 感谢你们的支持和鼓励。奶奶, 高龄逗哏和广播担当, 希望您九十三 活成三十九, 永远健康。表哥表嫂们, 你们永远把我当成需要罩着的妹妹, 谢谢你们每一次的热情招待 和不间断的亲切慰问。鱼和戎, 作为我的时尚顾问和心灵导师, 有你们这些先天乐观的发小们也让我无 
论遇到什么困难都更有底气了。爸妈, 感谢你们, 虽然对我未来的设想被完全颠覆, 但还是支持我实现 了小小的人生梦想。希望你们能不服老, 找到还能为之奋斗的目标, 和我一起过好接下来的精彩人生。

邓颖

2020 年 4 月 17 日 
About the author 


\section{Curriculum Vitae}

Ying Deng was born in Changzhou, China on November $3^{\text {rd }}$, 1988. After completing education in Hua Luogeng Middle School of Jiangsu Province, she started a bachelor's degree in Nanjing Forestry University majoring in Bioengineering (Biopharmacy direction) from 2006. After successfully graduated in 2010 followed by two years of industrial experience as an antibacterial test engineer, she moved to the Netherlands in 2013 to continue her education at Wageningen University and Research (WUR). In the master

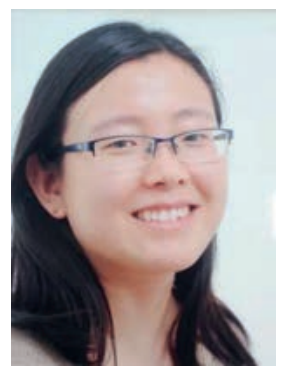
program, she showed strong interest in food immunology after following the course Food Related Allergies and Intolerance. Additionally, she joined the International Genetically Engineered Machine Competition (IGEM) team of WUR, which ran a synthetic biology project about Aspergillus niger. Later, she started a thesis about the expression of recombinant cashew nut allergen Ana o 3 in E. coli and Pichia in Food Biobased Research (FBR) of WUR. After an internship at Nestle Research Center (Lausanne) studying the role of gut microbiota in diarrhea using bioinformatic technology, she finished the master of Food Biotechnology in the summer of 2015.

Directly after her master graduation, she started a PhD thesis in FBR under the supervision of Prof. Harry Wichers. During her PhD, the author studied the physicochemical impact of processing on dietary proteins from aspects of digestion, intestinal uptake and immunological responses. Major milk allergen $\beta$-lactoglobulin was used in the study thoroughly with two other proteins lysozyme and thyroglobulin for their slightly different properties. A set of physicochemical methods were used to study the structure change of protein. In addition, digestibility and transportation of protein after processing was studied based on an in vitro digestion model and a Caco-2 cell monolayer model. THP-1 monocytederived macrophages and dendritic cells were used to support the immunological response research, using qPCR, ELISA, flow cytometry and microarray methods. Advance statistical analysis and basic programming skills ( $R$ language) were included to find order in the huge amounts of data.

By the first half of the year 2020, she worked as a research assistant in the Human Nutrition and Health group of WUR for Dr. Klaske van Norren next to working on finalizing her PhD research. She was involved in a project aiming to provide inside in improving feed efficiency in piglets after weaning based on histology and nutritional analysis.

\section{Contact information:}

Email: deyishwo@qq.com

Linkedin: https://www.linkedin.com/in/deng-ying-a3891282/ 


\section{List of publications}

H. Zenker, A. Ewaz, Y. Deng, H. Savelkoul, J. van Neerven, N. de Jong, H. Wichers, K. Hettinga, M. Teodorowicz (2019) "Differential Effects of Dry vs. Wet Heating of $\beta$-Lactoglobulin on Formation of sRAGE Binding Ligands and sIgE Epitope Recognition." Nutrients 11(6), 1432

Y. Deng, C. Govers, S. Bastiaan-Net, N. van der Hulst, K. Hettinga, H. Wichers (2019). "Hydrophobicity and aggregation, but not glycation, are key determinants for uptake of thermally processed $\beta$ lactoglobulin by THP-1 macrophages." Food Research International 120: 102-113

Sarker, S. A., B. Berger, Y. Deng, S. Kieser, F. Foata, D. Moine, P. Descombes, S. Sultana, S. Huq, P. K. Bardhan, V. Vuillet, F. Praplan and H. Brussow (2017). "Oral application of Escherichia coli bacteriophage: safety tests in healthy and diarrheal children from Bangladesh." Environmental Microbiology 19(1): 237-250.

Sarker, S. A., S. Sultana, G. Reuteler, D. Moine, P. Descombes, F. Charton, G. Bourdin, S. McCallin, C. Ngom-Bru, T. Neville, M. Akter, S. Huq, F. Qadri, K. Talukdar, M. Kassam, M. Delley, C. Loiseau, Y. Deng, S. El Aidy, B. Berger and H. Brussow (2016). "Oral Phage Therapy of Acute Bacterial Diarrhea with Two Coliphage Preparations: A Randomized Trial in Children from Bangladesh." Ebiomedicine 4: 124-137.

ten Buren, E. B. J., M. A. P. Karrenbelt, M. Lingemann, S. Chordia, Y. Deng, J. J. Hu, J. M. Verest, V. C. Wu, T. J. B. Gonzalez, R. G. A. van Heck, D. I. Odoni, T. Schonewille, L. van der Straat, L. H. de Graaff and M. W. J. van Passel (2014). "Toolkit for Visualization of the Cellular Structure and Organelles in Aspergillus niger." Acs Synthetic Biology 3(12): 995-998. 


\section{Overview of completed training activities}

Discipline specific courses $\quad$ Year

Conference, Innate host defence and Infections, Utrecht, the Netherlands a 2016

Symposium, Postgraduate Food Fraud (WFSR) ab 2016

Short Term Scientific Mission, COST Action FA1402, Belgrade, Serbia 2016

Course, Food Protein (6th edition), Copenhagen, Denmark 2016

$\begin{array}{ll}\text { Symposium, PhD Symposium (WPC) }{ }^{\text {bc }} & 2017\end{array}$

Symposium, Round table "PUFA - MICROBIOTA - IMMUNE HEALTH" (VLAG) ${ }^{\text {b }} 2017$

Conference, 4th ImpARAS conference, Naples, Italy ${ }^{d} \quad 2018$

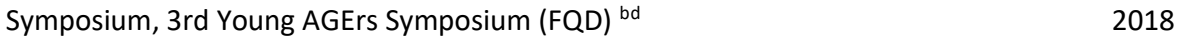

Symposium, "The Brave New World of Smart Data \& Semantics in the Life 2019

Conference, CRISPRcon ${ }^{b} \quad 2019$

General courses and activities Year

Course, PhD week (VLAG), Baarlo, the Netherlands ${ }^{d} \quad 2016$

Course, Reviewing a Scientific Paper (WGS) ${ }^{\text {b }} 2016$

Lunch lecture, "Science Communication" (VLAG) ${ }^{\text {b }} 2016$

Course, The Essentials of Scientific Writing and Presenting (WGS) ${ }^{\text {b }} 2016$

Course, Project and time management (WGS) ab 2017

Course, Teaching and supervising Thesis students (WGS) bd 2017

Course, Philosophy and Ethics of Food Science and Technology (WGS) ${ }^{\text {b }} 2017$

$\begin{array}{ll}\text { Event, Career day (WUR) }{ }^{\text {b }} & 2017\end{array}$

$\begin{array}{ll}\text { Symposium, Publish for Impact (WUR Library) }{ }^{\text {b }} & 2017\end{array}$

Course, Workshop: How to beat procrastination (4TU.CEE) ${ }^{\text {b }} 2018$

\section{Optional courses and activities}

Preparation of research proposal 2015

Research meetings, Weekly group meetings ${ }^{\text {bd }} \quad$ 2015-2019

$\begin{array}{ll}\text { PhD excursion, Japan ad } & 2016\end{array}$

$\begin{array}{ll}\text { PhD excursion, Austria and Italy }{ }^{\text {ad }} & 2018\end{array}$

100 years WUR project, Cross pollinations: Art meets Science! bd 2017

Talents group work, UNLEASH Innovation Lab, Shenzhen, China ad 2019

\section{Teaching obligations, courses}

Food Related Allergies and Intolerances (FCH-21806) 2016

Immunomodulation by Food and Feed (CBI-50806) 2016-2019

\section{Teaching obligations, students}

Master students, 6 months each, 4 students 
WFSR: Wageningen Food Safety Research

COST: European Cooperation in Science and Technology

WPC: Wageningen PhD Council

VLAG: Graduate School Food Technology, Agrobiotechnology, Nutrition and Health Sciences

FQD: Chair group Food Quality and Design

SSB: Chair group Systems and Synthetic Biology

WGS: Wageningen Graduate Schools

WUR: Wageningen University and Research

4TU.CEE: 4TU.Centre for Engineering Education

$\mathrm{FCH}$ : Chair group Food Chemistry

CBI: Chair group Cell Biology and Immunology

a poster presentation

${ }^{\mathrm{b}}$ Wageningen, the Netherlands

${ }^{\mathrm{c}}$ convener

d oral presentation 


\section{Colophon}

The work in this thesis was performed at Wageningen UR FBR (FQHE group). The research described in this thesis was conducted under scholarship granted by the China Scholarship Council (No. 201507720020). Financial support from Wageningen University for printing this thesis is gratefully acknowledged.

Cover design: Marie Declerfayt \& Ying Deng

Printing: Digiforce-Proefschriftmaken.nl 
